\title{
Mechanisms of Metal Release from Contaminated Coastal Sediments
}

\author{
by \\ Linda H. Kalnejais \\ B.Sc.(Hons), University of Western Australia, 1993 \\ B.E.(Hons), University of Western Australia, 1995 \\ Submitted in partial fulfillment of the requirements for the degree of \\ Doctor of Philosophy \\ at the \\ MASSACHUSETTS INSTITUTE OF TECHNOLOGY \\ and the \\ WOODS HOLE OCEANOGRAPHIC INSTITUTION
}

September, 2005

(C)MMV Linda H. Kalnejais

All rights reserved.

The author hereby grants to MIT and WHOI permission to reproduce paper and electronic copies of this thesis in whole or in part and to distribute them publicly.

Signature of Author ....... Joint Program in Oceanography Massachusetts Institute of Technology and Woods Hole Oceanographic Institution

June 17,2005

Certified by

Dr. William R. Martin Thesis Supervisor

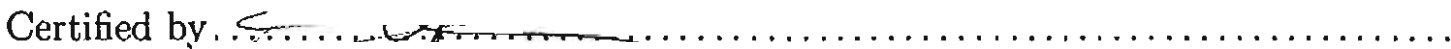
Professor Roger Francois Thesis Supervisor

Accepted by

Dr. Timothy I. Eglinton

Chair, Joint Committee for Marine Chemistry and Geochemistry

Senior Scientist.

Woods Hole Oceanographic Institution 


\title{
Mechanisms of Metal Release from Contaminated Coastal Sediments
}

\author{
by
}

\author{
Linda H. Kalnejais
}

\author{
Submitted to the Department of Marine Chemistry and Geochemistry, \\ Massachusetts Institute of Technology-Woods Hole Oceanographic Institution, \\ Joint Program in Oceanography \\ on June 17, 2005, in partial fulfillment of the \\ requirements for the degree of \\ Doctor of Philosophy
}

\begin{abstract}
The fate of trace metals in contaminated coastal sediments is poorly understood, yet critical for effective coastal management. The aim of this thesis is to investigate and quantify the mechanisms leading to the release of silver, lead and copper across the sediment-water interface. Two contrasting sites were investigated, a heavily contaminated site in Boston Harbor and a less impacted, offshore site in Massachusetts Bay. High-resolution porewater and solid phase samples were collected in each season to determine the diagenetic cycles and chemistry controlling the fate of these metals. The trace metals are scavenged by iron oxyhydroxides and released to the porewaters when these oxides are reduced. At the strongly reducing site in Boston Harbor, there is seasonal transfer of trace metals from oxide phases in winter, to sulfides phase in summer. At the Massachusetts Bay site, due to the lack of sulfide, the metals are focused into the surface oxide layer, giving a solid phase enrichment. There is a diffusive flux of copper to the water column throughout the year, while silver is released only in winter. Lead is strongly scavenged and is rarely released to the overlying waters. Analysis of reduced sulfur compounds in the porewaters has shown that there is also a significant flux of these strong ligands to the overlying waters. Polysulfide species enhance the solubility of copper within the porewaters. Sediment resuspension fluxes were quantified using an erosion chamber. Sediment resuspension leads to enhanced release of dissolved metals and is especially important in redistributing contaminants as the first particles to be eroded are enriched in trace metals. The total release of dissolved metals from the sediments by diffusion and sediment resuspension is estimated to be $60 \%$ and $10 \%$ of the riverine flux for copper and lead respectively. With continued pollution control reducing the discharge of metals from other sources, the benthic release of metals will become increasingly important terms in the metal budget of Boston Harbor.
\end{abstract}




\section{Acknowledgments}

I have so many people to thank for both helping me with my work and making the last few years so enjoyable. It has been a privilege to be part of the MIT-WHOI Joint Program. I especially want to thank my advisor Bill Martin, who allowed me to work independently and to pursue my own interests, yet was always very giving with ideas, resources and advice. My co-advisor Roger Francois has always been very encouraging and I am very grateful for the long hours in cold rooms that he spent with me sectioning sediments. My Committee has also been a wonderful source of knowledge and guidance. Jim Moffett encouraged me to pursue the thiol measurements and very generously gave me practically exclusive use of his HPLC. Tina Voelker inspired me with her Aquatic Chemistry class and has given me great advice and many insightful comments. I am very grateful for Peter Traykovski's expertise on sediment resuspension and his willingness to help a chemist investigate physical processes. Finally I would like to thank the chair of my defense, Dan McCorkle for his guidance and insight. I would also like to thank Dan for replying to an email back in 1998 and encouraging me to come to the Joint Program. I am also very grateful for the opportunity to work with Phil Gschwend at MIT when I first joined the Joint Program.

There is no way I could have undertaken this work with out the technical help, support and generosity of many people. I especially want to thank Joanne Goudreau, for teaching and guiding me in many aspects of lab work, running nutrient samples, deploying benthic chambers and being generally invaluable. Jennifer Morford gave me guidance when I started trace metal analysis and was a wonderful help at the start of this project with methods and advice. I would like to thank Michael Bothner of the USGS for his invaluable belp in sample collection, enthusiasm, encouragement and generosity with resources. I would also like to thank the team of divers who carefully collected cores for me, especially Lary Ball, Dann Blackwood and Michael Casso. Michael also helped extensively with the field sampling and organization. Matt Reuer provided me with thorough instructions on the Co-APDC method I used extensively. Many people have helped me with instruments and analytical techniques; Dave Schneider gave me a great deal of help with the ICP-MS, Delia Oppo generously allowed me to use her GFAAS and Bob Nelson taught me the thiol derivatization method. Rich Signell of the USGS spent considerable time re-running his hydrodynamic model to calculate shear stresses. Margaret Sulanowska was always a wonderful source of advice and drew several figures for me at short notice. I would also like to thank Meg Tivey for a great deal of advice and encouragement. I am grateful to Meg and Wolfgang Bach for providing me with an office in their lab, as well as great coffee and a supportive atmosphere. I also greatly appreciate the fabulous support of Lucinda Gathercole and Sheila Clifford. Brian Schroeder, Becky Belastock, Ellen Roosen, Dan Rogers and probably many more people I have failed to mention, all provided me with help over the years.

A great team of people have helped me section cores, spending many hours in the glove bag, including Mike Bothner, Jennifer Morford, John Crusius, Heidi Fuchs, Mea Cook, Nick Drenzek, Tracy Quan, Emilie Slaby, Sharon Hoffman, Sandy Baldwin, Susan Brown-Leger, Francois Lacan, Pieter Van Beek and Sejal Patel.

I have benefited scientifically as well as had alot of fun with my fellow chemistry students, especially Tracy Quan, Emilie Slaby, Rachel Wisniewski, Helen White, Cara Santelli, Bridget Berquist, Ana Lima, Amy-Marie Accardi-Dey, Nick Drenzek, Rachel Stanley and Paul Craddock. My friends in Boston and in Woods Hole have made these last six years so enjoyable - Mea, Heidi, Jeff, Kristy, Oscar, Charlie, Jessica, Carlos, Adam, Jim, Dirk, 
Heather, Michael, Sarah, Patty, Boa, Susan, Gary, Lara and Bruno. I have been sailing with Dottie Crossley for the last five years and she has been a source of inspiration, local knowledge and a great sailing instructor. I am so glad my great Perth friends, Andrew Houlding and Kate Murphy also decided to live in this hemisphere and that we have been able to meet up on many occasions. I have been so lucky to have fabulous housemates in Woods Hole and Boston; Margaret, Rhea, Cara, Joe, Sejal, Susan, Lara and Eoghan have all been great friends as well as a family away from home. I especially want to thank Margaret who has given me a great deal of help while writing my thesis and is a wonderful friend and great handstand coach.

Most of all I want to thank my family; my mother, father and sister, Rita, have been a constant source of encouragement and support. I am very fortunate to have such a loving family.

Finally I want to thank the dedicated people in the Academic programs Office, John Farrington, Judy McDowell, Julia Westwater and Marsha Gomes for all their help over the years. I also want to thank Malcolm Robb, Dave Heggie and Jorg Imberger, Australian scientists I was fortunate to work with, who strongly encouraged me to pursue further studies in the US. I am so glad they did.

This work is a result of research sponsored by the NOAA National Sea Grant College Program Office, Department of Commerce, under Grant. No. NA16RG2273, Woods Hole Oceanographic Institution Sea Grant Project Nos. 1-01-22227310 and 1-01-22227338. The views expressed herein are those of the author and do not necessarily reflect the views of NOAA or any of its subagencies. Additional funding was provided by the University of Western Australia Hackett Scholarship, the United States Geological Survey under Cooperative Agreement Number 00HQAG0001 and the National Science Foundation under Grant OCE-0220892. 


\section{Contents}

1 Introduction 11

2 Sediment Sampling and Trace Metal Analytical Methods 17

2.1 Introduction . . . . . . . . . . . . . . . . . 17

2.2 Sample Sites and Sediment Collection . . . . . . . . . . . . 17

2.3 Sediment Sectioning and Porewater Extraction . . . . . . . . . . . . 19

2.3.1 Solid Phase Analysis . . . . . . . . . . . . . . . . . 21

2.3.2 Sediment Sampling Artifacts . . . . . . . . . . . . . 22

2.4 Porewater Analytical Method . . . . . . . . . . . . . . . . . . . . 23

2.4 .1 Isotope Dilution $\ldots \ldots \ldots \ldots . \ldots \ldots \ldots$

2.4 .2 Standards and Solutions . . . . . . . . . . . . . . . 27

2.4 .3 Co-Precipitation Method . . . . . . . . . . . . 28

2.4 .4 The Co-APDC reaction . . . . . . . . . . . . . . 29

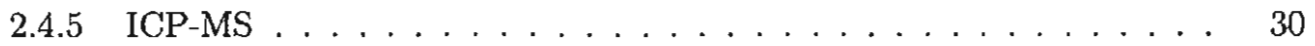

2.4.6 Data Collection Method . . . . . . . . . . . . . . . . . 32

2.4 .7 Data Processing . . . . . . . . . . . . . . . . . . 32

2.4 .8 Blank Correction . . . . . . . . . . . . . . . . 34

2.4 .9 Isotope Ratio Determination $\ldots \ldots \ldots \ldots$

2.4.10 Detection Limits . . . . . . . . . . . . . . . . . . 37

2.4.11 Mass Fractionation Corrections . . . . . . . . . . . . . . . 38

2.4 .12 Precision and Long-term Ratio Stability . . . . . . . . . . . 38

2.4.13 Accuracy and Precision of the Co-Precipitation Method . . . . . 42

2.4.14 Verification of the Co-APDC Method for Porewaters . . . . . . . 43

2.4 .15 Standard Additions $\ldots \ldots \ldots . \ldots . \ldots . \ldots 44$ 
2.5 Conclusions ........................ 46

3 Seasonal Cycling of Silver, Lead and Copper in Coastal Sediments $\quad 47$

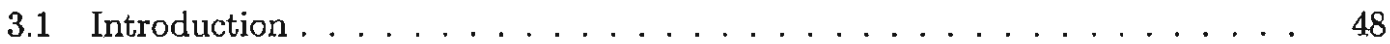

3.1.1 Metal Selection and Metal Chemistry . . . . . . . . . . . . 49

3.1 .2 Study Area . . . . . . . . . . . . . . . . . 52

3.2 Methods . . . . . . . . . . . . . . . . . . 53

3.2 .1 Sample Collection ........................ 53

3.2 .2 Benthic Chambers . . . . . . . . . . . . . . . 54

3.2.3 Porewater and Solid Phase Analysis . . . . . . . . . . . . . 55

3.3 Results and Discussion . . . . . . . . . . . . . . . . . . . 57

3.3.1 Sediment Redox Conditions . . . . . . . . . . . . . . . . 57

3.3 .2 Hingham Bay . . . . . . . . . . . . . . . . . . 58

3.3.3 Massachusetts Bay . . . . . . . . . . . . . . . . . 65

3.3.4 Trace Metal Solid Phase Distributions . . . . . . . . . . . . . 73

3.3.5 Trace Metal Porewater Distributions . . . . . . . . . . . . 74

3.3 .6 Hingham Bay . . . . . . . . . . . . . . . . . . 75

3.3.7 Massachusetts Bay . . . . . . . . . . . . . . . . . . . . . . . 98

3.3.8 Calculated Porewater Fluxes across the sediment-water interface . . 101

3.3.9 Benthic Chamber Data . . . . . . . . . . . . . . 106

3.3 .10 Comparison Between Sites . . . . . . . . . . . . . . . . 108

3.3.11 Conclusions . . . . . . . . . . . . . . . . . . . 115

3.4 Compilation of Porewater Data . . . . . . . . . . . . . . . . 118

3.4 .1 Additional Figures . . . . . . . . . . . . . . . 124

4 Reduced Sulfur Compounds in Porewaters 139

4.1 Introduction . . . . . . . . . . . . . . . . . . . . . . . . 139

4.2 Methods . . . . . . . . . . . . . . . . . . . . 141

4.2 .1 Study Areas . . . . . . . . . . . . . . . . . 141

4.2.2 Sample Collection and Processing . . . . . . . . . . . 141

4.2 .3 Thiol Analytical Technique . . . . . . . . . . . . . . . 141

4.3 Results and Discussion . . . . . . . . . . . . . . . . . 145

4.3.1 Derivatization Results . . . . . . . . . . . . . . . . . . 145 
4.3.2 Sediments as a Source of Reduced Sulfur Compounds . . . . . . . 153

4.3.3 Trace Metal Speciation in Porewaters . . . . . . . . . . . . 156

4.4 Future Work . . . . . . . . . . . . . . . . . . . . . . . 158

4.5 Conclusions . . . . . . . . . . . . . . . . . 160

5 The Role of Sediment Resuspension in the Remobilization of Metals from $\begin{array}{lc}\text { Coastal Sediments } & 163\end{array}$

5.1 Introduction . . . . . . . . . . . . . . . . . . . . . . . . 164

5.2 Materials and Methods . . . . . . . . . . . . . . . . . . . . . . . 169

5.2.1 Erosion Chamber Selection and Design Modifications . . . . . . 169

5.2 .2 Erosion Chamber Calibration . . . . . . . . . . . . . 170

$5.2 .3 \quad$ Field Sites . . . . . . . . . . . . . . . . . . . . 172

5.2 .4 Sampling . . . . . . . . . . . . . . . . . . . 174

5.2 .5 Erosion Experiments . . . . . . . . . . . . . . . . . . 174

5.2 .6 Incubation Experiments . . . . . . . . . . . . . . . . 176

5.2 .7 Sample Analysis . . . . . . . . . . . . . . . . . . 177

5.3 Results and Discussion . . . . . . . . . . . . . . . . . . . . 178

5.3.1 Erosional Characteristics . . . . . . . . . . . . . . 178

5.3.2 Erosion Depths . . . . . . . . . . . . . . . . . . . 180

5.3.3 Solute Release . . . . . . . . . . . . . . . . . . . 180

5.3.4 Particle Release . . . . . . . . . . . . . . . . . . . . . . . 198

5.3 .5 Incubations . . . . . . . . . . . . . . . . . . . 209

5.3.6 Temporal Variation of Shear Stresses . . . . . . . . . . . . . 217

5.3.7 Total Metal and Nutrient Fluxes to Boston Harbor . . . . . . . . . 220

5.3 .8 Conclusions . . . . . . . . . . . . . . . . . . . . . . . 226 


\section{Chapter 1}

\section{Introduction}

The long term fate of pollutants released to the environment is of considerable ecological and economic importance. The primary transport pathways for pollutants are rivers, groundwater, atmospheric transport and ocean outfalls, which ultimately discharge into estuaries and coastal margins. Once in the coastal zone particle reactive contaminants, such as metals and hydrophobic organic compounds, become associated with suspended solids and are subject to settling processes that deposit them into the sediments. However due to dynamic biogeochemical and physical processes active in the coastal zone, sediments are not necessarily a permanent repository for contaminants. With current legislative controls restricting pollutant discharge to the environment, the sediments now represent a potentially important source of pollutant species to coastal waters.

Three primary mechanisms drive the release of chemical species from sediments; (1) molecular diffusion of solute phases across the interface, (2) upward advective flows of porewaters, and (3) bulk mixing of sediment and porewater into the water column. Diffusive transport is a molecular-level process driven by a concentration gradient across the sedimentwater interface. Advective flows in fine grained sediments can be generated by benthic organisms irrigating their burrows. Bulk mixing can be due to biological activity, sediment resuspension processes, and sediment slumping or human activities such as dredging.

The relative importance of each of these mechanisms in coastal sediments is not fully understood. Benthic exchange processes in the coastal zone are poorly constrained compared to those in the deep ocean. In shallow waters extreme spatial and temporal variability of factors such as organic matter flux, temperature and macrofauna activity strongly in- 
fluence diffusive and advective exchange processes. Coastal sediments are also subject to fluid flows of varying magnitude and duration due to tidal and meteorological forcing. In previous studies the interaction between sediments and fluid flow was not considered, yet energetic flow events are likely to be important in mobilizing contaminants. Due to the logistical difficulties associated with sampling during intense flow conditions, such as storms and hurricanes, episodic events are undersampled. This represents a serious limitation to the data that is currently available.

The focus of this thesis is on the mechanismas that lead to the remobilization of trace metals from contaminated sediments. Metals, unlike some organic pollutants and nutrients, do not degrade into other forms. The long term fate of metals in the coastal zone is either permanent burial in the sediment or transportation to offshore waters. To make predictions about the fate of metals discharged to the environment it is critical to understand the chemical and physical processes occurring in the sediments. Silver, lead and copper were investigated in detail. High concentrations of these well-known contaminants are found globally in polluted sediments. Silver is one of the most toxic metals [Ratte, 1999], yet a complete understanding of the geochemical processes that cycle silver in the sediment does not exist. Silver, lead and copper span the range of metal behaviors found in the ocean. Silver has a nutrient-like profile, lead has a scavenged profile, and copper shows behavior intermediate between the two. The range of chemical properties observed with these metals provides a basis from which to assess the fate of other contaminant metals.

An interdisciplinary approach is taken in this thesis to address the limitations of our current knowledge. Traditional sediment geochemical methods of porewater sampling and benthic chamber deployments are combined with erosion chamber experiments, an approach more typical of the engineering community. With these techniques I have investigated two contrasting coastal sites in Massachusetts, a heavily contaminated site in Boston Harbor and a less impacted site offshore in Massachusetts Bay. Together they span the range of geochemical environments expected in coastal regions with fine-grained sediments.

In order to quantify solute fluxes from sediments, the concentration of the trace metals in porewaters must be accurately determined. Existing analytical methods were modified to allow high precision measurements on small volumes of porewater. The trace metal analytical procedure developed for this study is described in Chapter 2. Full investigation of the potential interferences from the complex porewater matrix are also discussed. By intro- 
ducing an isotope dilution technique we have significantly improved the precision associated with trace metal analysis in porewaters.

The processes driving diffusive exchange across the sediment-water interface are investigated in Chapter 3. In the past, diffusive exchange has been quantified with porewater sampling or benthic chamber deployments on only one or two occasions. However, in the coastal zone this approach does not provide adequate temporal coverage to understand the geochemical processes driving the exchange. I have addressed the effect of temporal variation in Chapter 3 by sampling on a seasonal basis for nearly three years. The improved sampling frequency has revealed a seasonal variation in metal fluxes, which has a significant effect on the magnitude of diffusive exchange. The diffusive flux of copper is calculated to be about $20 \%$ of the riverine input to Boston Harbor and thus an important term to understand in the regional copper budget.

In Chapter 3 I have not only quantified the diffusive fluxes, but have also investigated the chemical pathways and metal cycling that control both the seasonal diffusive flux and the long-term distribution of metals in the sediments. At both the Harbor and offshore sites, porewater profiles indicate that iron oxyhydroxides adsorb the trace metals, providing a strong coupling between the iron redox cycle and the metal behavior. The reduction of iron oxyhydroxides leads to the release of adsorbed metals to the porewaters. At the offshore site the released metals are re-scavenged by other iron oxyhydroxide particles in the oxic zone above, resulting in a net upwards flux that generates an enrichment of metals in the surface layers of the sediments. In contrast, the Harbor site is a strongly reducing environment with a distinctive seasonal oscillation in the depth of redox zones. This oscillation drives a different metal cycle. In spring, trace metals are released to the porewaters due to the reduction of iron oxyhydroxides. As the reducing conditions increase over summer, the released silver is precipitated out as a sulfide phase and the iron and trace metal porewater cycles are decoupled. The cycle is completed over winter as the redox horizons move back downwards and the metal sulfides precipitated over summer are oxidized and re-scavenged by iron oxyhydroxides.

While Chapter 3 shows that the flux of metals from the sediments is significant, the fate of these metals once they have crossed the sediment-water interface is unknown. Strongly complexed metals can resist oxidation and scavenging and are thus likely to persist in the water column. The goal of Chapter 4 was to identify potential strong ligands in the 
sediments and to assess their importance for metal complexation and therefore long-term fate. Reduced sulfur compounds, thiols (organic compounds with an -SH functional group) and polysulfides were measured due to their very strong affinity for silver, lead and copper(I). Results of Chapter 4 are preliminary but they suggest that reduced sulfur compounds are found within the sediments at concentrations that enhance the solubility of copper. In addition, high resolution porewater profiles and flux calculations indicate that there is a diffusive release of these species across the sediment-water interface that may be an important source of strong metal binding ligands to the waters of Boston Harbor. As the ligand flux is in excess of the trace metal flux, it is likely that the metals released from the sediments are complexed by these sediment-derived ligands. The ligand excess is sufficiently great that if the ligands resist oxidation within the water column, then benthicderived ligands will also impact the speciation of other metals within the water column of Boston Harbor.

The release of metals due to bulk mixing driven by sediment resuspension is quantified with an erosion chamber in Chapter 5. A laboratory based erosion chamber was used to impose shear stresses at the sediment-water interface that ranged from quiescent conditions to very strong storm conditions. The impact of moderate and extreme fiuid flows has thus been quantified for the first time. The results indicate that once the erosion threshold of the sediments is exceeded there is a release of materials from both the solute and solid phases into the water column. As noted in Chapter 3, the benthic metal cycle in Massachusetts Bay leads to a trace metal enrichment in the surface sediments, enhancing the importance of sediment resuspension at this site.

A single strong storm or hurricane in Boston Harbor will mobilize more solid phase metals into the water column than are discharged from other sources, including rivers and sewage effluent, in an entire year. If the resuspended solid phase is maintained in solution by energetic flow conditions, incubation experiments suggest that $5 \%$ of the silver, $6 \%$ of the copper and negligible lead and iron are released to the dissolved phase over 90 hours. Sediment resuspension processes are thus responsible for transferring a fraction of the silver and copper from the solid phase to the more bioavailabile and mobile dissolved phase within the water column. By coupling the flux measurements from the erosion chamber with a hydrodynamic model prediction of the temporal variability of shear stresses it is estimated that over a year, sediment resuspension in Boston Harbor contributes a flux of dissolved 
copper and lead of up to $40 \%$ and $10 \%$ of the riverine (dissolved + particulate) input respectively.

The total benthic release of dissolved metals to Boston Harbor has been quantified in more detail than at any other site and includes estimates of the diffusive flux and flux due to sediment resuspension. From the work in both Chapters 3 and 5 it is found that the sediments contribute up to $60 \%$ of the riverine copper llux to Boston Harbor. Due to the strong scavenging the sediments are a negligible source of lead. The sediments are also a source of dissolved silver, but as other inputs have not been quantified the importance of benthic release to the Harbor silver budget cannot be assessed. Nonetheless, this study has shown that benthic inputs of silver and copper to the coastal zone are significant. Due to pollution controls the discharge of metals from other major sources such as rivers and sewage effluent will continue to decline. The benthic release will become an increasingly important term in the metal budgets of coastal waters and so must be considered in the long-term management of contaminated sediments.

This thesis has made significant progress towards understanding the mechanisms and the magnitude of benthic silver, lead and copper fluxes in the costal Massachusetts. Contributions of this thesis include: (1) measuring porewater profiles of these metals on a seasonal basis, (2) determining that iron oxyhydroxides are the main carrier phase of metals, (3) identifying patterns of metal cycling in sediments under different redox conditions, (4) understanding the geochemistry that drives the temporal variation in metal fluxes, (5) quantifying fluxes and identifying chemical pathways of metal release due to sediment resuspension, and (6) determining that the sediments are a potentially important source of glutathione and polysulfides to overlying waters. Although these contributions are derived from analysis of Boston Harbor and coastal Massachusetts, they cover a wide range of geochemical conditions and can thus be applied to other coastal regions. The magnitude of benthic fluxes and the dominant mechanisms, which I have identified in this thesis, will aid managers in both assessing the long-term fate of metals in contaminated marine sediments and in designing remediation strategies.

In order to further refine our understanding of metal remobilization from contaminated sediments in the coastal zone, finer temporal and spatial measurements are required. In particular, to address the long-term aspects of resuspension events it is necessary to sample the overlying water column during storms and hurricanes. Realistically, this will only be 
accomplished with the development of permanently moored, trace-metal clean, automatic samplers. As the sediment sampling methods used in this thesis are labor intensive, it will also be desirable to develop in-situ sediment sampling techniques suitable for analyzing trace metals. 


\section{Chapter 2}

\section{Sediment Sampling and Trace Metal Analytical Methods}

\subsection{Introduction}

The aim of this thesis is to determine the geochemical pathways cycling lead, silver and copper in coastal sediments. Analysis of the concentrations in the porewater phase provides a sensitive indicator of the reactions occurring within the sediments and of the mechanisms driving the metal cycling. Porewater sampling is challenging and prone to artifacts due to sampling techniques and analytical methods. The goal of this chapter is to describe the sampling methods that were employed to ensure that sediment samples were collected in as close to an undisturbed manner as possible and that the porewaters extracted from the sediments were representative of the in-situ concentrations. The analytical methods to determine the metal concentrations in the porewater are then described as well as a series of tests that were undertaken in order to verify the accuracy and precision of the porewater analytical technique.

\subsection{Sample Sites and Sediment Collection}

Two fine-grained sediment sites in coastal Massachusetts were investigated during this study. The first site is located in the north-western portion of Massachusetts Bay, $9 \mathrm{~km}$ offshore in $30 \mathrm{~m}$ of water. This site is referred to as the Massachusetts Bay (MB) site. The second site is in the south-east corner of Boston Harbor, in Hingham Bay in a water depth of 5 
m. This site is referred to as the Hingham Bay (HB) site. The contamination history and the sedimentary and chemical nature of each site is described and investigated in detail in Chapters 3 and 5 so will not be discussed here. The sediment collection methods are however described here.

Removing a core from the sediment immediately introduces a potential disturbance and no method currently available for sampling porewaters leaves the sample completely undisturbed. High resolution in-situ techniques such as polyacrylamide gel probes either disrupt the equilibrium within the porewaters in order to concentrate a sufficient amount of metal for analysis, and so do not actually measure the porewater concentration, as is the case with the diffusional gradient in thin films (DGT) technique used by Davison et al. [1994] or do not provide sufficient sample to analyze low levels of multiple trace metals, as is the case for the diffusional equilibration technique (DET) used by Davison et al. [1991]. Voltammetric techniques [Luther et al., 1998] can give fine-scale information on a range of redox active species such as $\mathrm{Fe}, \mathrm{Mn}$ and $\mathrm{O}_{2}$, but instrumentation to determine the trace metals of interest here at $\mathrm{pM}$ to $\mathrm{nM}$ concentrations is not available. The traditional method of slicing and centrifuging [Elderfield et al., 1981] was thus employed in this study. The great advantage of this method over either the gel probe methods or voltammetry is that additional species such as nutrients, alkalinity, sulfide, iron and manganese can all be determined on the same porewater sample, so that a much more comprehensive understanding of the sedimentary redox environment and diagenetic processes can be obtained. As lead, silver and copper are all found at trace levels in the sediments, it is essential to understand the dominant porewater species that control the chemical environment, and thus strongly influence the trace metal reactions within the sediments.

At both sites sediment cores were collected to ensure minimum disturbance of the sediment-water interface. Sediment samples from the Massachusetts Bay site were collected with a hydraulically damped gravity corer [Bothner et al., 1998] that ensures a slow rate of penetration. Recovered cores were a maximum of $70 \mathrm{~cm}$ long. Divers using SCUBA collected cores from the shallow Hingham Bay site and the maximum core length was 40 $\mathrm{cm}$. Core barrels for each site were polycarbonate with a diameter of $10 \mathrm{~cm}$. The core barrels were cleaned with a phosphate-free detergent, rinsed with MQ water, then rinsed quickly with $5 \% \mathrm{HCl}$ and rinsed a further 5 times with $\mathrm{MQ}$ water. The barrels were then dried under a laminar flow hood and capped with end caps for storage. 
At each site collected cores were placed on ice immediately after collection and brought back to shore. Cores for porewater and solid phase analysis were sectioned as soon as logistically possible. For Hingham Bay samples the cores were driven back to WHOI and sectioned in a $4^{\circ} \mathrm{C}$ walk-in refrigerator, with processing started within 4 hours of sample collection. Massachusetts Bay samples were sectioned at ambient air temperature in a van at the Coast Guard dock in Boston, so sectioning was started within 4 hours of sample collection. At least two cores were sectioned at high resolution for porewaters on each cruise, except for the Hingham Bay October 2003 cruise, when only a single core was sectioned at high resolution.

\subsection{Sediment Sectioning and Porewater Extraction}

Once the cores arrived at the sectioning location the waters overlying each core were sampled in duplicate for all species that would also be measured in the porewaters. The cores were always transported from the site of collection with no air space to prevent aeration of the core and to minimize disturbance due to sloshing water. After the overlying water was sampled, the water in the core barrel was siphoned until only $10 \mathrm{~cm}$ of overlying water remained above the sediment-water interface. The core was then placed in a core extrusion stand, designed to rigidly hold the core barrel in place. The core was then quickly encased in a disposable glove bag (I2R research, New Jersey). The water level was lowered to 2 cm above the sediment-water interface and the glove bag sealed and filled with high purity nitrogen gas. The glove bag was flushed three times with nitrogen to ensure that the oxygen concentration within the bag was negligible.

The remaining overlying water was removed with a syringe and the sediment-water interface raised to be flush with the top of the core barrel. Sediment was then carefully transferred with a plastic spoon to nitrogen-flushed scintillation vials. The sediment was sampled as uniformly and horizontally as possible. The scintillation vials were filled completely, so that the depth of the sediment section could be determined volumetrically, as well as from a ruler mounted on the side of the core. The $25 \mathrm{ml}$ scintillation vial represents a sampling depth of about $3 \mathrm{~mm}$ and provides sufficient porewater to analyze most species of interest. After six scintillation vials were filled with sediment, the sampling resolution was reduced and $50 \mathrm{ml}$ nitrogen-flushed centrifuge vials were filled completely, giving a res- 
olution of $6 \mathrm{~mm}$. Once 12 samples had been collected the resolution was generally reduced, with 1 or $2 \mathrm{~cm}$ sections of sediment sliced from the core and a portion of each slice transferred into $50 \mathrm{ml}$ nitrogen-flushed centrifuge tubes. 24 sediment samples were collected. The scintillation vials and centrifuge tubes were sealed as soon as they were filled. At regular intervals the samples were passed outside the glove bag and centrifuged at $8000 \mathrm{rpm}$ for 10 minutes. All sampling materials used in sectioning the sediment, such as centrifuge tubes, scintillation vials and spoon, were MQ soaked and dried in a laminar flow hood prior to use. On completion of the centrifuging, the samples were returned quickly to another nitrogen-filled glove bag.

All materials used for filtering and storing the porewaters were subject to strict cleaning protocols. Any plasticware to come in contact with a sample for trace metals was acid cleaned. The acid cleaning protocol entailed 4 days of soaking in $2 \mathrm{~N} \mathrm{HCl}$ at $60^{\circ} \mathrm{C}$ followed by 5 rinses with MQ and drying in a laminar flow hood. This protocol was used for all plasticware except for the syringes $(10 \mathrm{ml}$ latex free, $B-D)$ used to sip the porewater from the centrifuge tube and filters. The syringes were soaked in $2 \mathrm{~N} \mathrm{HCl}$ at room temperature for 4 days as higher temperatures distorted the plastic barrel. The $0.45 \mu \mathrm{m}$ polysulfone membrane acrodisc filters (Pall Gelman, $25 \mathrm{~mm}$ ) for metal analyses were soaked in $2 \mathrm{~N} \mathrm{HCl}$ for 2-3 hours, then rinsed with at least $30 \mathrm{mls}$ of $\mathrm{MQ}$ water until the $\mathrm{pH}$ of the filtrate was equal to that of the MQ water. The filters were then dried by passing $10 \mathrm{mls}$ of air through them. Any item used to sample the porewater for nutrients and alkalinity were MQ soaked, MQ rinsed and air dried.

The centrifuged samples were uncapped in the glove bag and the porewater divided into aliquots so that it could be analyzed for various species. The procedure for filtering and allocating the porewater was as follows; an acid-cleaned $10 \mathrm{ml}$ syringe (B-D) was used to sip $4.5 \mathrm{mls}$ of sample from the centrifuge tube. The porewater in the syringe was then passed through an acid-cleaned $0.45 \mu \mathrm{m}$ filter into a $4 \mathrm{ml}$ HDPE acid-cleaned vial. The first 0.3-0.5 mls of solution to pass through the filter was discarded. The $4 \mathrm{ml}$ vial was pre-spiked with $40 \mu \mathrm{L}$ of optima concentrated nitric acid on the morning of the sample collection, so that the sample was acidified to $\mathrm{pH}<1$ immediately after filtration.

The remainder of the porewater was sipped into a $10 \mathrm{ml}$ MQ soaked syringe (B-D, latex stopper) and filtered through a. MQ cleaned $0.45 \mu \mathrm{m}$ polysulfone membrane acrodisc filter, again discarding the first $0.3-0.5 \mathrm{mls}$. $1 \mathrm{ml}$ of sample was transferred to a $1 \mathrm{ml}$ syringe (B- 
D, no pre-cleaning) and stoppered for total carbon dioxide determination. The remainder of the porewater was transferred to a $25 \mathrm{ml}$ Evergreen plastic vial (Evergreen Industries). Subsamples were pipetted from this vial to give samples for alkalinity, sulfate and sulfide (both preserved with zinc acetate and 6\% sodium hydroxide solution). In Hingham Bay samples were also taken for thiol analysis (derivatized with monobromobimane, see Chapter 4 for more details). The remaining sample in the evergreen vial was for ammonium, nitrate+nitrite, phosphate and silicic acid analysis.

All samples were analyzed as soon as possible. Total carbon dioxide samples were stored in ice water in the dark until analyzed the following day on a Lycor infra-red gas analyzer (analyses done by Dr Bill Martin). The samples for nutrients $(3 \mathrm{mls})$ were refrigerated and analyzed within 2 days of collection with a Technicon autoanalyzer and the method of Glibert and Loder [1977] (analyses done by Joanne Goudreau). Samples for alkalinity (0.5-1 ml) were stored at room temperature and analyzed within 1-4 days by an automated Gran titration [Gieskes, 1973] (also analyzed by Joanne Goudreau). Samples for sulfide $(0.5 \mathrm{mls})$ and sulfate $(0.5 \mathrm{mls})$ were refrigerated, sulfide was analyzed within a week by the spectrophotometric method of Cline [1969] and sulfate was analyzed within a month by ion-chromatography. The sulfate samples were first passed through a Dionex OnGuard IIH $1 \mathrm{ml}$ ion exchange cartridge to remove the zinc preservative and then analyzed with a Dionex 500 ion chromatograph with an AS15 anion column (analyses done by Rebecca Belastock). Samples for thiols were stored at $-40^{\circ} \mathrm{C}$ and analyzed within a month. Samples for trace metals were stored double bagged in the refrigerator until analyzed.

\subsubsection{Solid Phase Analysis}

The solid phase samples remaining after the extraction of porewater were either refrigerated at $-40^{\circ} \mathrm{C}$ for future solid phase analysis or were freeze dried. At least one core per cruise was freeze dried at USGS as soon as possible. The dried sediment samples were then disaggregated in an agate mortar and pestle and counted with a GeLi detector for ${ }^{210} \mathrm{~Pb}$ and ${ }^{234} \mathrm{Th}$ (counting and analysis by Joanne Goudreau and Bill Martin). After the counting was completed (including recounts) the samples were ground further and sent to Dr Paul Lamonthe at USGS in Colorado for solid phase metal analyses. A total digestion method with aqua regia, HF and perchloric acid followed by ICP-MS quantification was used to determine the concentration of 40 metals in the sediments [Briggs and Meier, 1999]. 


\subsubsection{Sediment Sampling Artifacts}

The most concerning potential artifact that sediment slicing and centrifuging can introduce is sample oxidation [Morford et al, 2003]. To assess the susceptibility of the sediments and the porewater metal concentrations to oxidation a series of test was undertaken during the Hingham Bay October 2003 cruise. The Hingham Bay site is the most reducing of the study sites so represents the warst case scenario for an oxidation artifact. A slice of sediment from $15-20 \mathrm{~cm}$ (concentration of sulfide $=300 \mu \mathrm{M}$ ) was homogenized in the glove bag by stirring in a 1 liter beaker with a teflon rod. Six centrifuge tubes were filled with the sediment. Two of the tubes were capped immediately and stored in the glove bag until they were centrifuged, so representing the normal method of processing. The treatments for the remaining 4 centrifuge tubes were designed to represent various mishaps that could lead to the sample being accidentally exposed to the atmosphere. Two of the centrifuge tubes were removed from the glove bag and left uncapped for 30 minutes prior to centrifuging. The other set of two were capped immediately but after centrifuging they were uncapped and exposed to the atmosphere for 30 minutes. The final two centrifuge tubes were filled with sediment from the beaker after the beaker had been removed from the glove bag for 30 minutes. This final treatment represents an extreme condition that did not occur during processing of sediments for this thesis. The porewater concentrations for each of these treatments are shown in Figure 2-1 (analyzed by the method described below).

The porewater concentrations of silver are not significantly impacted if the centrifuge tubes containing the sediment sample are exposed to up to 30 minutes of oxygen. Very high levels of silver (higher than ever measured in a porewater profile) are however released to the porewaters when the entire sediment is exposed to oxygen. Exposure of the centrifuge tubes to the atmosphere does elevate the porewater concentrations of lead and copper by $40 \%$ and $80 \%$ respectively, above the concentration measured in the standard treatment. Whether the exposure is before or after centrifuging does not seem to matter. As with silver a strong release occurs when the entire sediment is exposed to oxygen. This series of tests indicates the extreme care that must be taken to ensure that the centrifuge tubes are well capped when removed from the glove bag. The caps on the centrifuge tubes were always checked once the tubes were removed from the glove bag, so that, except in the unlikely case of a defective cap, the samples would never have been exposed to the atmosphere for 

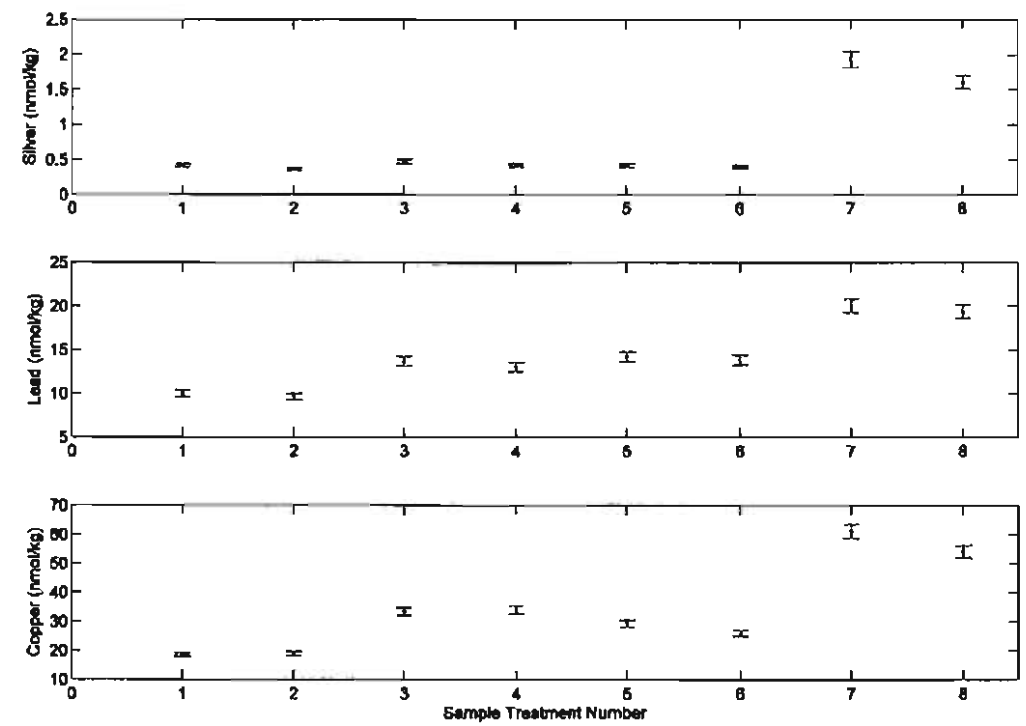

Figure 2-1: Results of oxidation tests conducted on sediment from Hingham Bay October $200315-20 \mathrm{~cm}$. Treatments $1-2=$ normal processing, $3-4=$ samples in centrifuge tubes exposed to the atmosphere for 30 minutes after centrifuging, 5-6 = samples in centrifuge tubes exposed to the atmosphere for 30 minutes before centrifuging, $7-8=$ entire sediment exposed to the atmosphere for 30 minutes. Error bars are a single standard deviation.

half an hour. These tests thus represent worst case scenarios.

\subsection{Porewater Analytical Method}

The challenges associated with the analysis of trace metals in porewater include the limited volume of sample available, the complex seawater matrix and the potentially high concentrations of metal chelators due to high levels of dissolved organic carbon and sulfide species. An additional issue is that analyses of trace metals, especially lead and copper, are prone to contamination from sampling devices, storage bottles, atmospheric dust and reagents. A pre-concentration and matrix removal method is thus required for the metals of interest in porewaters to ensure that the metal concentrations are sufficiently elevated above blank levels and that the chosen analytical technique is not compromised by low sensitivity due to interference from the salts in seawater.

The pre-concentration method chosen was based on the method developed by Matthew Reuer, for the analysis of lead and cadmium in corals from the co-precipitation technique introduced by Boyle and Edmond [1977]. The method concentrates metals by co-precipitation with cobalt ammonium pyrrolidinedithiocarbamate. The dithiocarbamate ligand, with its 
two donor sulphur atoms, forms strong complexes with many metals [Hulanicki, 1967]. With pyrrolidine as a substituent many of the complexes formed are insoluble in water. By the addition of excess cobalt to a sample, precipitation of Co pyrrolidinedithiocarbamate is used to strip other soft metals from solution, effectively concentrating them in the solid phase. The advantage of this technique lies in the fact that the dithiocarbamate ion decomposes in acidic solution, so that the precipitate can be easily digested, giving a solution containing the concentrated trace metals that can be analyzed by an appropriate technique. Other published methods for the determination of multiple elements in small volumes of porewaters have relied on an APDC and diethylammonium diethyldithiocarbamate organic extraction [Rivera-Duarte and Flegal, 1996]. This technique relies on several chloroform extractions for each sample. As well as requiring volatile and carcinogenic reagents this method also requires more sample handling and manipulation. The Co-APDC represents a simpler and safer pre-concentration technique.

The addition of an isotope spike to the sample prior to co-precipitation acts as an internal standard to compensate for any losses in recovery during extraction and digestion. The additional benefit of isotope addition is the enhanced precision obtained from isotope dilution mass spectrometry (IDMS). Due to these benefits an inductively coupled plasmamass spectrometer (ICP-MS) was used to analyze the porewater extracts.

\subsubsection{Isotope Dilution}

Isotope dilution mass spectrometry is an extremely sensitive and precise analytical method [Heumann, 1986]. IDMS relies on the addition of an accurately known isotope spike to the sample. The isotope spike consists of an enriched stable isotope, with the enriched isotope generally an isotope with low natural abundance. The true isotopic ratio, $R$ of the spiked sample is then given by

$$
R=\frac{N_{\text {samp }} A_{\text {samp }}^{h}+N_{\text {spike }} A_{\text {spike }}^{h}}{N_{\text {samp }} A_{\text {samp }}^{l}+N_{\text {spike }} A_{\text {spike }}^{l}}
$$

where knowing the isotope ratio $R$, enables the number of moles in the sample $N_{s a m p}$ to be calculated from a knowledge of a number of moles of added spike, $N_{\text {spike }}$ and the isotopic abundance $A(\%)$, of the heavy isotope $h$ and the lighter isotope $l$ in both the sample and the spike. To optimize the amount of spike to be added, the errors associated with each term in equation 2.1 can be propagated. Assuming that the isotopic abundances 
Table 2.1: Spike and natural isotope abundances for $\mathrm{Ag}, \mathrm{Cu}$ and $\mathrm{Pb}$.

\begin{tabular}{llll}
\hline Element & Isotope & Spike abundance (\%) & Natural Abundance (\%) $^{2}$ \\
\hline $\mathrm{Ag}$ & ${ }^{107} \mathrm{Ag}$ & $0.74 \pm 0.05$ & 51.839 \\
& ${ }^{109} \mathrm{Ag}$ & $99.26 \pm 0.05$ & 48.161 \\
$\mathrm{Cu}$ & ${ }^{63} \mathrm{Cu}$ & $0.30 \pm 0.02$ & 69.17 \\
& ${ }^{65} \mathrm{Cu}$ & $99.70 \pm 02$ & 30.83 \\
$\mathrm{~Pb}$ & ${ }^{204 \mathrm{~Pb}}$ & 0.0302 & 1.4 \\
& ${ }^{206} \mathrm{~Pb}$ & 0.20603 & 24.1 \\
& ${ }^{207} \mathrm{~Pb}$ & 0.68958 & 22.1 \\
& ${ }^{208} \mathrm{~Pb}$ & 99.10136 & 52.4 \\
\hline
\end{tabular}

${ }^{1}$ Spike abundances for $\mathrm{Ag}$ and $\mathrm{Cu}$ provided by Oak Ridge National Laboratory and $\mathrm{Pb}$ spike abundances from Dr Blusztajn, WHOl. ${ }^{2}$ Rosman and Taylor [1999]

in the sample and spike lead to negligible errors the standard deviation, $s$ in $N_{\text {samp }}$ can be determined as [Heumann, 1998]:

$$
s^{2}\left(N_{s a m p}\right) \approx s^{2}\left(N_{s p i k e}\right)+f^{2}(R) s^{2}(R)
$$

To minimize the error in $N_{s a m p}$ the error multiplication factor $f(R)$ must be minimized. $f(R)$ is given by

$$
f(R)=\frac{\left[\left(A^{h} / A^{l}\right)_{s a m p}-\left(A^{h} / A^{l}\right)_{s p i k e}\right] R}{\left[R-\left(A^{h} / A^{l}\right)_{s a m p} \mid\left[\left(A^{h} / A^{l}\right)_{s p i k e}-R\right]\right.}
$$

and the $f(R)$ function for silver, copper and lead are shown in Figure 2-2, using the isotopic compositions given in Table 2.1, and assuming that the isotopic abundance in the sample is natural abundance. The minimum of the $f(R)$ function occurs at

$$
R_{\text {opt }}=\left[\left(A^{h} / A^{l}\right)_{s a m p}\left(A^{h} / A^{l}\right)_{s p i k e}\right]^{1 / 2}
$$

However, from Figure 2-2 it is evident that due to the broad curvature of the $f(R)$ function, the minimum error spans a range of $N_{\text {samp }} / N_{\text {spike }}$ values. The concentration of the isotope spike for the porewaters was thus designed to fall with the broad minimum, so that a range of expected porewater concentrations would still lie within the minimized error region. 

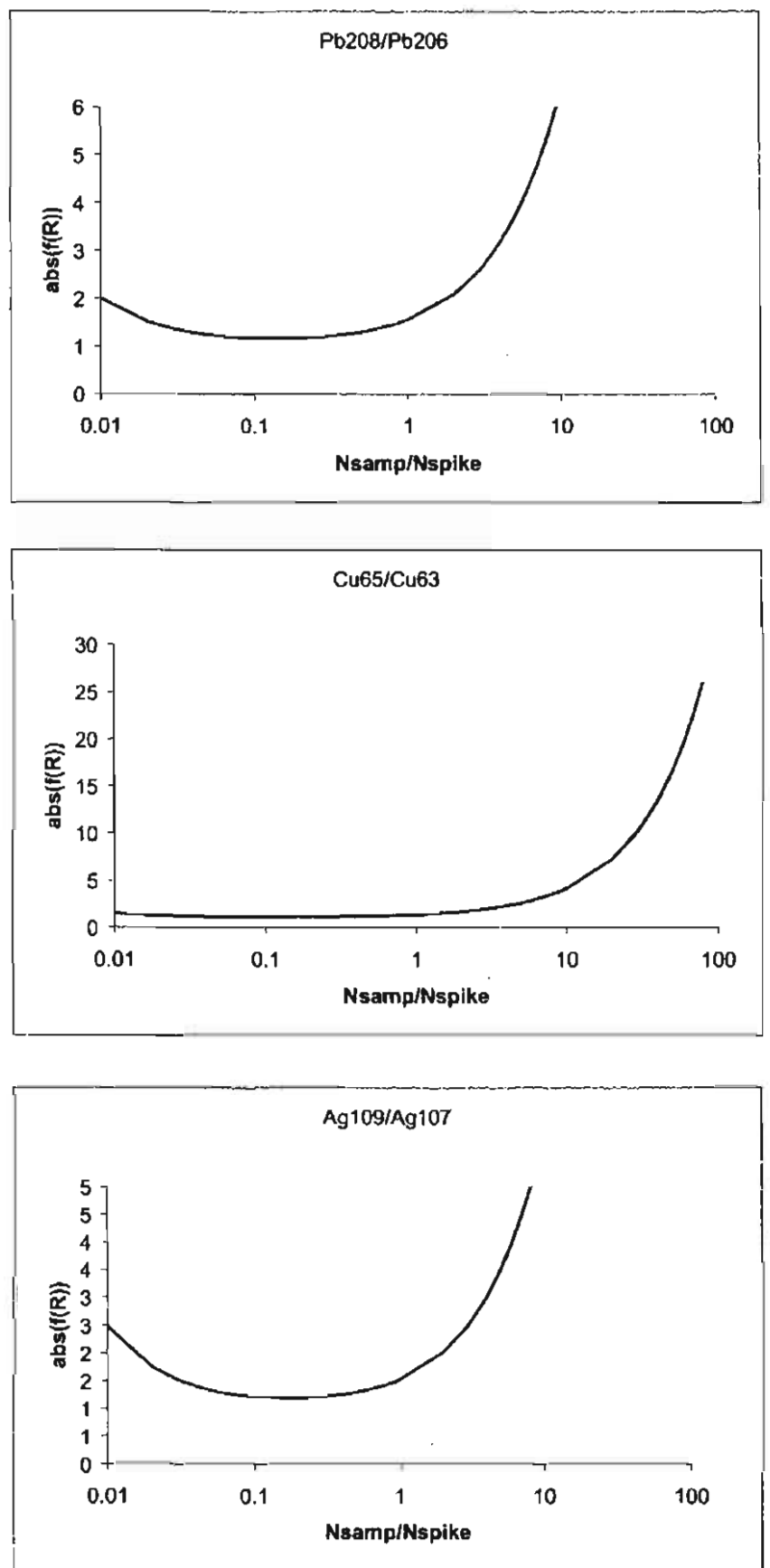

Figure 2-2: Optimization of the isotope dilution technique. The error multiplication factor $f(R)$ for silver, copper and lead. The optimum spike level is when $f(R)$ is a minimum. 


\subsubsection{Standards and Solutions}

All processing of samples and standards was undertaken in a clean room with overhead HEPA filtered air conditioning. Samples were only opened in a class 100 laminar flow hood within the clean room to avoid airborne contamination. All solutions were prepared in 18.2 $\mathrm{M} \Omega$ Milli-Q (MQ) treated water. All plasticware was leached in $2 \mathrm{~N}$ reagent grade $\mathrm{HCl}$ at $60^{\circ} \mathrm{C}$ for 4 days followed by 5 rinses with MQ water and dried in a laminar flow hood. Teflon plasticware was used wherever possible and cleaned with at least a three day soak in fresh aqua regia followed by $6 \mathrm{hrs}$ in boiling 50\% nitric acid and a final five rinses in MQ water. All standards were prepared from Alpha Aesar single element primary standards that have an accuracy of $0.3 \%$. Standards were stored in aqua-regia leached teflon bottles. All acid solutions for the preparation of standards, instrument blanks and sample digestion or dilution were prepared from trace metal grade Fisher Optima nitric acid.

Isotope spikes for ${ }^{109} \mathrm{Ag}$ and ${ }^{65} \mathrm{Cu}$ were obtained from Oak Ridge National Laboratory (Oak Ridge, Tennessee) as Ag trimmings and $\mathrm{CuO}$ powder respectively. The ${ }^{208} \mathrm{~Pb}$ spike solution was provided by Dr Jurek Blusztajn from the Geology and Geophysics department. The elemental ratios of the spikes are provided in Table 2.1.

The ${ }^{109} \mathrm{Ag}$ and ${ }^{65} \mathrm{Cu}$ spikes were prepared by the dissolution of the supplied trimmings or powder in a $50 \% \mathrm{HNO}_{3}$ solution. Following complete dissolution, the solution was diluted to a $5 \% \mathrm{HNO}_{3}$ solution. The ${ }^{109} \mathrm{Ag},{ }^{65} \mathrm{Cu}$ and ${ }^{208} \mathrm{~Pb}$ spikes were combined at appropriate concentrations into a multispike solution, taking into account the concentrations of the other 2 elements in each spike solution. The concentrations of each element in the spike was verified by reverse isotope dilution. For reverse isotope dilution a highly accurate concentration and isotopic abundance of a standard is required, against which the spike is calibrated. For silver and copper the isotopic abundance of the Alpha Aesar standard solutions was assumed to be at the natural abundances given in Table 2.1. This assumption is not necessarily valid for the lead standard due to the wide variability of lead isotopic compositions. The isotopic composition of the lead standard solution was instead determined from calibration against the known isotopic composition of the National Institute of Standards and Technology (NIST) Standard Reference Material (SRM) 981, common lead isotope standard. The porewaters were all assumed to have isotope ratios at natural abundance, and this was confirmed by extraction of unspiked porewaters. 


\subsubsection{Co-Precipitation Method}

The method described below is closely based on the method developed by Matthew Reuer. Most of the cleaning steps and preparation of reagent solutions were performed as recommended. The quantities of reagents, sample handling and treatment of the pre-concentrated sample were modified to adapt the method to porewater analysis on the ICP-MS. The method is described below.

\section{Reagent Cleaning}

The APDC reagent was prepared by dissolving $2 \mathrm{~g}$ of solid APDC (Sigma-Aldrich) in 100 mls of MQ water. The solution was purified with five sequential extractions with chloroform (Fisher Scientific HPLC Grade) in a teflon separatory funnel. The $\mathrm{CHCl}_{3}$ contained pentene as a stabilizer, so was cleaned with ten extractions with MQ water, following the method of Rivera-Duarte and Flegal [1996]. The purified APDC solution was stored frozen when not in use.

The cobalt solution was prepared from a saturated cobalt chloride solution by initially dissolving $9 \mathrm{~g}$ of cobalt (III) chloride hexahydrate (Fisher Scientific $99.999 \%$ purity) in 25 mls of MQ and adding more solids until excess remained. The cobalt solution was purified with ion-exchange chromatography. $500 \mathrm{~g}$ of Biorad AG1x8 anion exchange resin (100 200 mesh chloride form) was cleaned with 5 overnight extractions on a shaker table in $6 \mathrm{~N}$ $\mathrm{HCl}$ (Fisher Optima) followed by 5 extractions in MQ. The cleaned resin was poured into an acid-cleaned glass chromatography column of height $700 \mathrm{~mm}$ and internal diameter 26.5 mm (Labglass Lurex, New Jersey). The column of resin was pre-conditioned with three bed volumes of trace element grade $6 \mathrm{~N} \mathrm{HCl}$. Ten mls of the saturated $\mathrm{CoCl}_{2}$ solution was then added to the top of the column and the Co was eluted with $2 \mathrm{~N} \mathrm{HCl}$. The middle of the Co peak was determined visually and collected in an acid cleaned quartz crystallizing dish. The $\mathrm{CoCl}_{2}$ solution was reduced to dryness on a hotplate under HEPA filtered air. The resultant purified salt was stored in a desiccator until the final solution was prepared. The cobalt solution used in the co-precipitation reaction was a $2.5 \mathrm{mM}$ solution of the purified salt, prepared in MQ water.

The Co-APDC precipitation requires a basic solution for maximum recovery of solids. Ammonium hydroxide is used to control the $\mathrm{pH}$. The $\mathrm{NH}_{4} \mathrm{OH}$ was purified using a headspace 
distillation technique in which a $250 \mathrm{ml}$ acid cleaned teflon bottle half filled with MQ water was partially immersed in $500 \mathrm{mls}$ of concentrated $\mathrm{NH}_{4} \mathrm{OH}$ solution (Seastar Chemicals) in a tightly sealed container. The container was gently shaken for 3 days, so that the partial pressure of ammonia in the sealed container was high, driving ammonia into the cleaned container until equilibrium was reached. The purified $\mathrm{NH}_{4} \mathrm{OH}$ solution was stored in the refrigerator.

\subsubsection{The Co-APDC reaction}

For porewater analysis $1400 \mu \mathrm{L}$ of acidified porewater is transferred to a $2 \mathrm{ml}$ teflon centrifuge vial (Upchurch Scientific, Washington) and spiked with $100 \mu \mathrm{L}$ of the isotope spike with a concentration designed to generate the isotope ratio for lead, silver and copper in the range required for error minimization. All spiking is done gravimetrically. The spike is allowed to equilibrate for at least $48 \mathrm{hrs}$ with the sample to allow the trace elements in the porewaters time to exchange with the isotopes.

After the equilibration period, $200 \mu \mathrm{L}$ of the $\mathrm{CoCl}_{2}$ solution, followed by $100 \mu \mathrm{L}$ of distilled ammonia is added to the spiked porewater. $100 \mu \mathrm{L}$ of $2 \%$ APDC is then added and the vial shaken. A green amorphous precipitate forms. The vials are centrifuged at $8,000 \mathrm{rpm}$ for 10 minutes to ensure all the precipitate settles to the bottom of the vial. The supernatant is then removed as completely as possible with an acid cleaned $6 \mathrm{ml}$ disposable, fine tipped plastic Pasteur pipette. The samples are then allowed to dry at least overnight in the laminar flow hood.

One day prior to the analysis of the samples the precipitates are digested. Complete digestion of the organic matrix is important to ensure optimal sensitivity on the ICP-MS. $25 \mu \mathrm{L}$ of hot concentrated nitric acid (Fisher Optima) is pipetted into each sample vial. The vials are capped and then heated to $250^{\circ} \mathrm{C}$ on a hotplate. The precipitate turns from a green solid to a green-brown solution. Upon heating, a pink cobalt residue turns to deep blue as the salt is dehydrated. A second $25 \mu \mathrm{L}$ addition of hot concentrate nitric acid is added and heated to ensure complete destruction of the APDC. After cooling the samples are diluted with $500-1000 \mu \mathrm{L}$ of MQ water and are then ready to be run on the ICP-MS.

For seawater, benthic chamber and exosion chamber samples the procedure is the same except that 4-10 mls of sample is required and the procedure is performed in a $12 \mathrm{ml} \mathrm{HDPE}$ centrifuge tube (Nalgene). Adequate removal of the seawater from the Co-APDC precipitate 
is more difficult with the larger test tubes, so that after the supernatant has been removed the precipitate is shaken with $M Q$ and re-centrifuged to remove more of the salts.

\subsubsection{ICP-MS}

The isotope ratios and concentrations of all metals were quantified using either an Element or Element2 (Finnigan-MAT, Bremen, Germany) high resolution ICP-MS. The Element was used until March 2003 and the Element2 was used from September 2003. Both instruments are a magnetic sector, double focusing mass spectrometer with a single electron multiplier. Both instruments have three resolution settings $(\mathrm{R}=\Delta \mathrm{m} / \mathrm{m}$ at $10 \%$ peak height); low resolution (Element $\mathrm{R}=300$, Element2 $\mathrm{R}=300$ ), medium resolution (Element $\mathrm{R}=3000$, Element2 $R=4000$ ) and high resolution (Element $R=7500$, Element2 $R=10000$ ). Silver and lead were scanned in low resolution. ${ }^{63} \mathrm{Cu}$ is subject to spectral interferences due to the formation of ${ }^{23} \mathrm{Na}^{40} \mathrm{Ar}^{+},{ }^{26} \mathrm{Mg}^{37} \mathrm{Cl}^{+},{ }^{25} \mathrm{Mg}^{38} \mathrm{Ar}^{+}$and ${ }^{24} \mathrm{Mg}^{23} \mathrm{Na}^{16} \mathrm{O}^{+}$molecular ions which occurs when a saltwater matrix is introduced into the argon plasma. Operation at medium resolution is sufficient to resolve the ${ }^{63} \mathrm{Cu}$ peak from this interference. Both ${ }^{63} \mathrm{Cu}$ and ${ }^{65} \mathrm{Cu}$ were thus determined in medium resolution. The ICP-MS operating conditions are provided in Table 2.2 and 2.3 .

Sample was introduced into both instruments with an ASX-100 micro-autosampler (CETAC Technologies). To generate a dried aerosol from the sample for introduction into the plasma a combination of a PFA microconcentric nebulizer and a membrane desolvation system is used (Element - MCN-6000, Element2 - Aridus, both from CETAC Technologies ). The nebulizer generates an aerosol of finely divided droplets that are swept into a spray chamber to create a more uniform droplet size. The vapor from the spray chamber is then swept into the desolvation system which consists of a heated porous fluoropolymer membrane system in which a counter current of argon drives the solvent to permeate through the membrane, leaving a dried aerosol. Both desolvation systems are designed to minimize sample consumption and to dry the sample and hence enhance sensitivity through the reduction of solvent-based interferences and oxide formation [Field et al., 1999].

The conditions were optimized before running on each day by tuning on a $0.1 \mathrm{ppb}$ solution of uranium and indium, so that the operating conditions differed slightly from day to day. Sensitivities were generally between $0.7-2 \times 10^{6} \mathrm{cps} / \mathrm{ppb}$ for ${ }^{238} \mathrm{U}$ and $4-9 \times 10^{5}$ cps/ppb for ${ }^{115}$ In in low resolution. 
Table 2.2: Data Acquisition Parameters ${ }^{1}$

\begin{tabular}{lll}
\hline Resolution & Low & Medium \\
\hline Isotopes monitored & ${ }^{107} \mathrm{Ag},{ }^{109} \mathrm{Ag},{ }^{206} \mathrm{~Pb}$, & ${ }^{63} \mathrm{Cu},{ }^{65} \mathrm{Cu}$ \\
Scan mode & E scan,${ }^{208} \mathrm{~Pb}$ & \\
Mass window (\%) & 5 & E scan \\
Samples per peak & 200 & 120 \\
Segment duration (s) & $0.2 / 0.4$ & 20 \\
Sample time (s) & $0.02 / 0.04$ & $1.2 / 0.96$ \\
Search window (\%) & 150 & $0.05 / 0.04$ \\
Integration window (\%) & 80 & 150 \\
Runs & 25 & 80 \\
Passes & 1 & 20 \\
Take up time (s) & 45 & 1 \\
Wash time (s) & $222 / 240$ & \\
Analysis Time & 1 min $57 \mathrm{~s} / 1$ min $45 \mathrm{~s}$ \\
Total time per sample & 6 min $24 \mathrm{~s}$ \\
\hline
\end{tabular}

${ }^{1}$ Where two values are given, the first refers to the Element operating conditions and the second to the Element2.

Table 2.3: Isotope-specific operating parameters ${ }^{1}$

\begin{tabular}{llll}
\hline Isotope & Magnet Mass & Mass Range & Settling Time (s) \\
\hline${ }^{107} \mathrm{Ag}$ & 106.905 & $106.896-106.913$ & 0.3 \\
${ }^{109} \mathrm{Ag}$ & 106.905 & $108.895-108.913$ & 0.001 \\
${ }^{206} \mathrm{~Pb}$ & 205.974 & $205.957-205.991$ & $0.130 / 0.055$ \\
${ }^{207} \mathrm{~Pb}$ & 205.974 & $206.958-206.993$ & 0.001 \\
${ }^{208} \mathrm{~Pb}$ & 205.974 & $207.959-207.993$ & 0.001 \\
${ }^{63} \mathrm{Cu}$ & 62.929 & $62.916-62.942$ & $0.191 / 0.300$ \\
${ }^{65} \mathrm{Cu}$ & 62.929 & $64.914-64.940$ & 0.001 \\
\hline
\end{tabular}

${ }^{1}$ Where two values are given, the first refers to the Element operating conditions and the second to the Element2. 
The operating parameters were selected in order to optimize the precision of each isotope ratio, within the constraint of limited sample size $(1000 \mu \mathrm{L})$. The precision of each isotope measurement is a function of the total time spent on each isotope per run, whether adjusted through the sampling time or number of points per peak [Poitrasson and Dundas, 1999]. For ratio measurements, however, the precision is also determined by the scanning rate, which needs to be of a sufficiently high speed to take into account fluctuations in signal intensity due to poor nebulization and sample introduction, plasma ficker and ionization variations [Vanhaecke et al., 1997]. The parameters in Table 2.2 represent a compromise between total analysis time, sufficient time per isotope and rapid peak scanning.

\subsubsection{Data Collection Method}

Both Element instruments are configured to accept up to 35 samples per autosampler run. A tray of 35 samples was thus loaded into the sampling carousel at a time. One tray is set up with 3 standards, followed by 2 acid blanks, 2 APDC blanks, and then 5-7 samples. Then 1 standard, $1 \mathrm{HNO}_{3}$ blank and 1 APDC blank are alternated between 7-9 samples, with the final sample always being a standard. This setup ensures that the sensitivity, precision and blank values can be assessed at the staxt of each run, and that every sample is well bracketed by both a standard and the appropriate blank.

Between each sample the system was rinsed for at least 3.5 minutes with $5 \% \mathrm{HNO}_{3}$. The last 120 seconds of this rinse was analyzed by the same analytical method as for the samples. This allowed any carryover between samples to be monitored. The wash acid was the same as that used for the $\mathrm{HNO}_{3}$ vial blanks.

\subsubsection{Data Processing}

In low resolution mode, the peak tops of ${ }^{107} \mathrm{Ag},{ }^{109} \mathrm{Ag},{ }^{206} \mathrm{~Pb},{ }^{207} \mathrm{~Pb}$ and ${ }^{208} \mathrm{~Pb}$ were electrostatically scanned. Only the central $5 \%$ of the peak was scanned to ensure data was collected only from the flat portion of the peak. Each run generates a spectrum for each mass, and 25 runs were performed for each sample analyzed. The data from each sample were downloaded using the Finnigan Results software. This raw data was processed in Matlab version 7.0.1 to remove any outliers from the data that may have resulted from, for example, poor nebulization and sample introduction, plasma flicker and premature consumption of sample.

With a mass window of $5 \%$ the number of samples per peak is 10 . For each run the 
average intensity across the mass range is calculated for each isotope. The mean sample intensity is then calculated as the average of the average of each run, as described in the following equation:

$$
I_{s a m p}=\left[\sum_{r=1}^{r=r \text { tot }} \sum_{m=1}^{m=n m a s s} I_{m r}\right] \frac{1}{r_{\text {tot }}} \cdot \frac{1}{n m a s s}
$$

where $I_{m r}$ is the intensity measurement at a particular mass for the run number, $r$. nmass is the number of masses per peak (10 in this case) and rtot is the total number of spectra generated (number of runs $=25$ ). The standard deviation is calculated as the standard deviation of all the data. Any values of $I_{m r}$ that fell outside of \pm 3 standard deviations from the mean were removed. The mean and standard deviation were then recalculated. This procedure was repeated twice. This generally resulted in the removal of 0 to 3 points for each isotope. In the case of obvious spikes or premature consumption of analyte, the appropriate number of points was removed to correct the problem. The final standard deviation that is reported and propagated in further calculations is calculated as the standard deviation of the cleaned averages of each run. This is consistent with the method used for calculating the average sample intensity and is the algorithm used by Finnigan (but without spike removal) to report data. The advantages of the statistics calculated in this manner is that short term fluctuations within a run are removed, so that the reported average and standard deviation reflect the intensities and precision over the entire time the sample is measured.

In medium resolution, 20 spectra each of ${ }^{63} \mathrm{Cu}$ and ${ }^{65} \mathrm{Cu}$ were collected. To resolve the ${ }^{63} \mathrm{Cu}$ peak a slit of fixed width is inserted into the ion path, producing a peak with a triangular shape. The sensitivity in medium resolution is reduced by a factor of about 14, compared to that of low resolution [Moens et al., 1995]. For comparable sensitivity and an appropriate isotopic ratio, ${ }^{65} \mathrm{Cu}$ was thus also run in medium resolution. To ensure that the isobaric interference was removed and to eliminate the low signal:noise portion of the peak, only $80 \%$ of each peak was integrated. The Finnigan software was used to do this integration.

Due to the shape of the peak, medium resolution is far more prone to errors due to changes in mass calibration over a run. The Finnigan software provides a 'lock mass' to compensate for drift. The ${ }^{40} \mathrm{Ar}^{40} \mathrm{Ar}$ dimer is monitored as the locked mass for this purpose and shifts in the mass calibration of the dimer are applied, with appropriate corrections for 
each mass, to all other peaks. Despite this correction, drift is still evident over several hours and regular monitoring of the signal is required to ensure that the peak still falls within the mass window. Correction required due to drift in the mass calibration or removal of outliers is done manually in Excel.

\subsubsection{Blank Correction}

The intensity measured for each sample includes contributions from the instrument blank and the reagent blank. This contribution needs to be removed before ratio calculations are performed [Heumann, 1998]. Due to the trace levels of metals in the sample, an accurate estimate of the blank values for each metal is essential. $5 \%$ nitric acid samples were run every 9-11 samples to give an estimate of the blank associated with the vials, acid and instrument. When running APDC extracts, APDC reagent blanks were also run every 911 samples to give the reagent + instrument blank for the co-precipitation method. The APDC blanks were determined by the addition of the reagents to sample vials with no sample and subsequent treatment of the precipitate as if it were a sample. The counts measured represent the metal concentrations in the APDC reagents + the instrument blank. Representative values for acid and APDC blanks are shown in Table 2.4.

The level of metals in the blank is the most important factor limiting the success of this method. The instrument blank is quite variable from day to day, as is evident from Table 2.4, and very dependent on the samples introduced by previous users and the cleaning routine employed before running. Silver had the most stable and low average levels in the acid blank, with a variation of less than a factor of 2 over a year. During the same time the average copper blank varied by over a factor of 30 . On a standard day $5 \% \mathrm{HNO}_{3}$ is flushed through the system for about two hours while the instrument is turned on and tuned. Wherever possible on the day prior to running, the Aridus desolvator is filled with warm $\mathrm{MQ}$ water, followed by $5 \% \mathrm{HNO}_{3}$, then $5 \% \mathrm{HCl}$ then warm $\mathrm{MQ}$ water again and allowed to sit overnight. This more rigorous cleaning has a marked impact on the blanks. The Aridus soaking was only introduced routinely in August 2004, and the vast improvement in the copper blanks between March and August are due to this more thorough cleaning. Field and Sherrell [2003] have attained the best detection limits achieved with a multi-element method in freshwater to date. By spending at least two days prior to running, flushing and cleaning the entire system, including the cones, torch, injector, nebulizer and all tubing, 
Table 2.4: Acid and APDC reagent blanks for the ELEMENT2 on representative run days in 2004 .

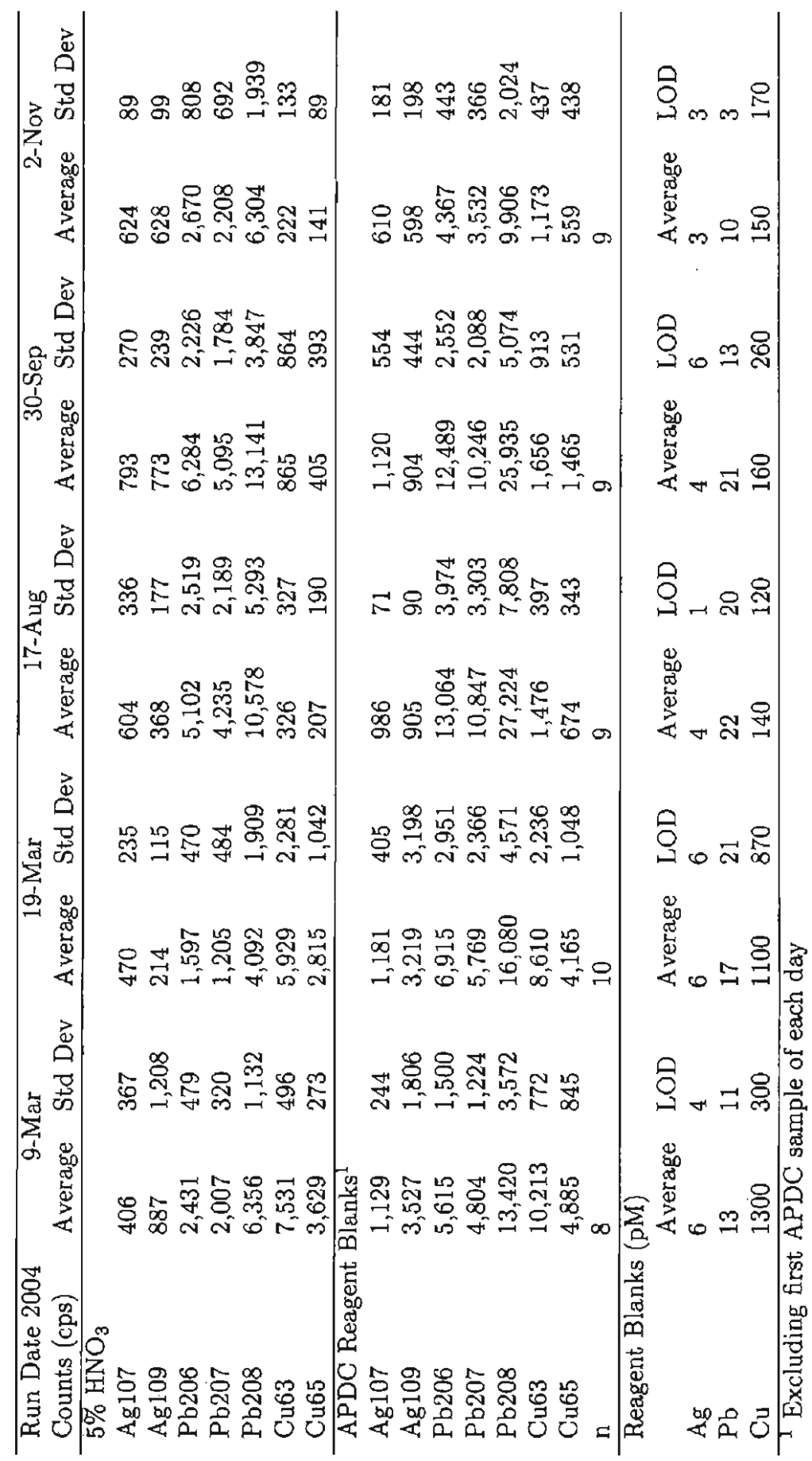


the blank level they report in a $3 \% \mathrm{HNO}_{3}$ solution for ${ }^{208} \mathrm{~Pb}$ is $117 \mathrm{cps}$ and for ${ }^{63} \mathrm{Cu}$ is 26 . The ICP-MS used in this case is an instrument dedicated to ultra-trace analysis. A more realistic blank level is reported by Poitrasson and Dundas [1999] that is on the order of 1000 cps for ${ }^{206} \mathrm{~Pb}$ on the Element and comparable to that achieved with the higher sensitivity WHOI Element2 ICP-MS after a thorough clean.

The APDC blank is higher than the acid blank for the three metals and also varies considerably between runs. The first APDC matrix sample to be aspirated into the ICPMS is often 2-6 times higher than the average values given in Table 2.4. This suggests that the change in matrix causes a desorption of ions from the internal surfaces of the nebulizer and desolvation system. Excluding the first APDC sample, the APDC blanks are generally 1-4 times higher than the acid blank. Analysis of the individual reagents indicates that the majority of blank was from the APDC reagent, though there is measurable copper in the cobalt reagent also.

The trace element concentrations of silver and lead in the blank are relatively small and range from 3-6 pM for silver and 10-22 pM for lead (Table 2.4). These values are similar to the blank concentrations achieved by Rivera-Duarte and Flegal [1996] for their porewater analyses. The concentration in the blank for copper was much more variable, largely due to the instrumental blank. A blank value of $1300 \mathrm{pM}$ for copper, which occurred in March 2004 is too high, so that a rigorous cleaning regime of the Aridus was instigated after this to ensure more acceptable and reproducible blank values. After the desolvation membranes were soaked and rinsed the blanks for copper were comparable to those of Rivera-Duarte and Flegal [1996].

\subsubsection{Isotope Ratio Determination}

The Element operating in 'E-scan' mode scans the acceleration voltage while keeping the magnetic field voltage constant. This avoids hysteresis effects in the magnet, and so allows rapid scanning across isotopes [Diemer et al., 2002]. Although a simultaneous determination was not possible, neighboring masses are scanned in quick succession to improve the precision of isotope ratios.

The isotope ratio of a sample was calculated from the data from which outliers were removed by the above cleaning process. For each run, the ratio was determined as the run 
average $\bar{I}_{r}$

$$
\bar{I}_{r}=\left[\sum_{m=1}^{m=n m a s s} I_{m r}\right] \cdot \frac{1}{n m a s s}
$$

of the heavy isotope $(h)$ divided by the run average of the light isotope $(l)$. Each run average was blank corrected prior to calculating the average. The blank value $I_{\text {blank }}$ used was appropriate for the sample matrix, either an APDC matrix or an acid matrix, and was the average blank for the rack of 35 samples $(n=5-8)$. The sample isotope ratio was then calculated as the average of the ratios from each run as shown in equation 2.7

$$
R=\left[\sum_{r=1}^{r=r t o t} \frac{\bar{I}_{r}^{h}(\text { sample })-I^{h}(\text { blank })}{\bar{I}_{r}^{l}(\text { sample })-I^{l}(\text { blank })}\right] \cdot \frac{1}{r t o t}
$$

\subsubsection{Detection Limits}

The detection limit for ID-ICP-MS is limited by three factors [Heumann, 1998]: (1) The level of the blank, (2) the precision of the isotope ratio measurement and (3) the minimum amount of an element that can be isolated. The first factor is the most important for the elements in this study, as silver, lead and copper are all commonly used metals and thus prone to contamination. The detection limit in IDMS when the blank is the most important factor is defined by [Heumann, 1998] as three times the standard deviation of the blank. The detection limits are also given in Table 2.4 for representative ICP days. The detection limit for silver was 4-6 pM and for lead was 3-21 pM. The detection limit for copper, once the high copper blank problems were improved, was $120-260 \mathrm{pM}$. The lead and silver are comparable to the detection limits of Rivera-Duarte and Flegal [1996], while for copper the detection limits are 2-3 times higher. That the detection limits have not been improved by changing from an ICP-MS with greater sensitivity than a graphite furnace atomic absorption spectrometer is because the concentration of trace metals in the instrument and reagent blank is the limiting factor. An ICP-MS and desolvation unit dedicated to trace metal analysis, more intensive cleaning of the APDC by extracting with carbon tetrachloride rather than chloroform, and use of a longer anion exchange column for cleaning the Co will enable blank improvements.

The blanks were less than $5 \%$ of the total counts for the lowest abundance (not isotopically enriched) isotope for all porewater analyses except for silver in Massachusetts Bay. The deeper samples for porewater in Massachusetts Bay, although above the limit of detec- 
tion, required a blank subtraction of up to $20 \%$ from the ${ }^{107} \mathrm{Ag}$ counts. If available, more than the standard $1400 \mu \mathrm{L}$ of porewater was extracted to try and improve this situation, but $1400 \mu \mathrm{L}$ was often all the porewater that was available for trace metal analysis.

\subsubsection{Mass Fractionation Corrections}

Mass fractionation leads to a deviation of the measured isotopic ratio from the true ratio. In the ICP-MS the most significant mass fractionation effect leads to a preferential loss of the lighter isotope. The primary cause for mass fractionation is attributed to space charge affects [Niu and Houk, 1996]. As the ion beam from the plasma passes through the skimmer, electrons are lost to the wall, leading to a charge separation in the beam, in which electrostatic repulsion cause the beam to expand. The degree of displacement from the beam axis is inversely dependent on the kinetic energy, and hence on the mass of the ion. Lighter isotopes are preferentially removed by the vacuum pumping systems, so further enhancing the discrimination against lighter isotopes.

Corrections for mass fractionation were made assuming a linear model of the form [Heumann, 1998];

$$
F F=1+\Delta m \varepsilon_{l i n}
$$

where the fractionation factor FF is applied to the measured isotope ratio (heavy isotope over light isotope)

$$
R_{\text {corrected }}=\frac{R_{\text {measured }}}{F F}
$$

The fractionation factor is calculated from $\Delta \mathrm{m}$, the mass difference between the isotope pair, and $\varepsilon_{l i n}$, the mass discrimination per amu. The value for $\varepsilon_{l i n}$ is determined for each day from the equation

$$
\varepsilon_{\text {lin }}=\left[\frac{\text { Rmeasured }}{\text { Rstd }}-1\right] \frac{1}{\Delta m}
$$

\subsubsection{Precision and Long-term Ratio Stability}

Standard solutions were run at the start of each sequence and at intervals of every 7-11 samples to monitor the variation in the fractionation factor as well as to monitor sensitivity and precision changes throughout the day. An example of the internal (within-sample) error of this standard is given in Table 2.5. The concentrations of the metals in this standard were not the same, but are in proportion to what was expected in porewaters. The concentrations 
Table 2.5: Internal precision (single sample) of a standard sample.

\begin{tabular}{lll}
\hline & $\% \mathrm{CV}$ & $\begin{array}{l}\text { Poisson } \\
\text { Predicted \%CV }\end{array}$ \\
\hline Ag107 & 2.12 & 0.11 \\
$\mathrm{Ag} 109$ & 2.45 & 0.12 \\
$\mathrm{Ag} 109 / \mathrm{Ag} 107$ & 1.56 & 0.16 \\
$206 \mathrm{~Pb}$ & 2.06 & 0.03 \\
$208 \mathrm{~Pb}$ & 2.15 & 0.02 \\
$\mathrm{~Pb} 208 / \mathrm{Pb} 206$ & 1.09 & 0.04 \\
$\mathrm{Cu} 63$ & 2.23 & 0.04 \\
$\mathrm{Cu} 65$ & 2.44 & 0.05 \\
$\mathrm{Cu} 65 / \mathrm{Cu} 63$ & 1.04 & 0.06 \\
\hline (\%CV $=\mathrm{Coefficient}$ of Variation $=$ standard deviation $/$ average & (100) \\
{$[\mathrm{Ag}]=1.4 \mathrm{nmol} / \mathrm{kg} ;[\mathrm{Pb}]=9.6 \mathrm{nmol} / \mathrm{kg} ;[\mathrm{Cu}]=220 \mathrm{nmol} / \mathrm{kg}$}
\end{tabular}

were raised above expected porewater concentrations to ensure good counting statistics and blank values that did not interfere with the determination of the fractionation factor. The minimum internal error is described by Poisson statistics, with $\sigma_{\text {counting }}=\sqrt{N}$, where $N$ is the number of counts. The error due to ion counting thus varies with concentration, and the contribution of this uncertainty to the total error is given in Table 2.5 .

For each element the uncertainty in the individual isotope is comparable to the uncertainty in the isotope ratio. Although this is predicted from Poisson statistics (with error propagation giving the error in a ratio as $\sigma_{\text {ratio }} / R=\sqrt{1 / N_{1}+1 / N_{2}}$, where $N_{1}$ and $N_{2}$ are the counts for each isotope and $\mathrm{R}$ is the ratio), it is obvious that counting errors do not account for most of the uncertainty observed. The contributions to the error include variations in nebulization, ionization, plasma stability and electronic noise. Calculating the ratio has improved the precision, so has reduced some of these variations. Poitrasson and Dundas [1999] report an uncertainty on a $5 \mathrm{nmol} / \mathrm{L} \mathrm{Pb}$ solution of $0.5 \%$ for the ${ }^{206} \mathrm{~Pb} /{ }^{208} \mathrm{~Pb}$ ratio. This is better than the results here, but they were not limited by sample volume, so had 750 runs per sample, took over 8 mins per sample, and just analyzed lead and thallium in low resolution. They thus had much better counting statistics. Poitrasson and Dundas [1999] found that the \%CV (Coefficient of Variation = standard deviation/average $\times 100$ ) was between three and ten times higher than those predicted by Poisson statistics and attributed a large part of the extra variations to the $\mathrm{MCN}-6000$ ultrasonic nebulizer. This unaccounted error fraction is similar for $\mathrm{Ag}$ but not $\mathrm{Pb}$ or $\mathrm{Cu}$. A difference in behavior between elements has been noted before. Field et al. [1999] observed that lead and iron 
Table 2.6: Replicate statistics for a standard solution analyzed during each ICP-MS run. An example of the reproducibility over a single day (from 29 June 2004) and the long term statistics are given. The year-long \%CV data are the \%CV for the averages from each ICP day.

\begin{tabular}{|c|c|c|c|c|c|c|c|c|}
\hline & Average & $\begin{array}{l}\text { Std Dev } \\
29 \text { June } 0\end{array}$ & $\begin{array}{l}\% \mathrm{CV} \\
4 \mathrm{Std} 1\end{array}$ & $\begin{array}{l}\text { Natural } \\
\text { Abundance }\end{array}$ & $\mathrm{FF}$ & $\varepsilon_{\text {lin }}$ & $\begin{array}{l}\% C V \\
2004\end{array}$ & $\begin{array}{l}\text { \%CV } \\
2002-2004\end{array}$ \\
\hline Agl07 & 176,797 & 3,280 & 1.9 & & & & & \\
\hline $\mathrm{Ag} 109$ & 166,785 & 2,777 & 1.7 & & & & & \\
\hline $\mathrm{Ag} 109 / \mathrm{Ag} 107$ & 0.94 & 0.02 & 2.12 & 0.929 & 1.0157 & 0.78 & 0.6 & 0.9 \\
\hline Pb206 & $1,213,934$ & 17,790 & 1.5 & & & & & \\
\hline $\mathrm{Pb} 208$ & $2,512,868$ & 30,731 & 1.2 & & & & & \\
\hline $\mathrm{Pb} 208 / \mathrm{Pb} 206$ & 2.07 & 0.03 & 1.25 & 2.2203 & 1.0023 & $0.150^{*}$ & 0.6 & 1.2 \\
\hline Cu63 & 414,990 & 3,741 & 0.9 & & & & & \\
\hline Cu65 & 190,518 & 1,710 & 0.9 & & & & & \\
\hline Cu65/Cu63 & 0.46 & 0.003 & 0.72 & 0.4457 & 1.03 & 1.5 & 1.2 & 1.4 \\
\hline
\end{tabular}

were more prone to spikes resulting from poor nebulizer or spray chamber operation than other elements.

The external precision, or precision between samples, is the more relevant descriptor of instrument precision than the internal precision [Gwiazda et al, 1998] as it represents the instrument reproducibility. Table 2.6 shows the results for the same standard solution as presented in Table 2.5 run several times per day as well as a long-term comparison. From Table 2.6 the far superior stability of the isotopic ratios compared to the individual counts is obvious when compared over several samples. The stability of the mean ratio over extended periods of time is also evident with the \%CV of the mean over several months, in the case of silver and lead being less than or equal to that for the \% CV for the same sample run several times on the same day. The long term external error for lead isotopes is less than that obtained by Poitrasson and Dundas [1999], who on a $5 \mathrm{nmol} / \mathrm{L} \mathrm{Pb}$ solution reported a \%RSD for the ${ }^{206} \mathrm{~Pb} /{ }^{208} \mathrm{~Pb}$ ratio of $0.15 \%$ over 4 months.

The external error is expected to encompass the internal error. So it is somewhat surprising that the external error for each isotope ratio is less than or equal to the internal error. This inconsistency has been reported in other ICP-MS studies as well. Gwiazda et al. [1998] attribute the discrepancy to the fact that the internal error is somehow over estimated and use the external error determined from repeated analyses of the same sample to determine the measurement error. The internal error may be overestimated due to the 


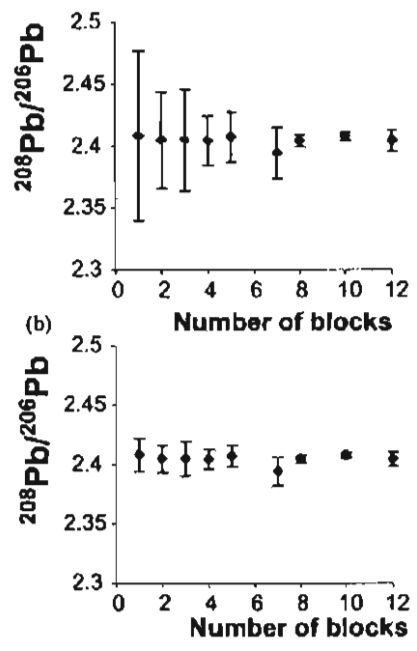

Figure 2-3: The calculated ${ }^{208} \mathrm{~Pb} /{ }^{206} \mathrm{~Pb}$ ratio of a single analysis of a standard as a function of the number of blocks used to calculate the average and standard deviation. Total number of runs $=25$. The error bars are the standard deviation. (b) As for (a) except the error bars are the standard error $(=\text { stdev/(no of runs/no of blocks })^{0.5}$ )

method of calculating the standard deviation. It is sometimes practiced in ICP-MS analysis to divide the total number of runs into several blocks and to calculate the ratio for each block. The internal error may be more representative if the size of the block is such that it encompasses the dominant periodic fluctuations in the signal. Indeed Figure 2-3 shows the decrease in \% CV as the number of blocks increases, while the calculated ratio remains stable. However when the standard error $(=s t d e v / \sqrt{n})$ is calculated, in order to normalize by the number of observation generating the mean ( $\mathrm{n}=$ number of runs/number of blocks) the impact of the number of blocks on the calculated internal error is significantly less. All the data have been calculated with a single block (ie 25 observations).

The long-term uncertainty in the lead isotope ratio exceeds the correction required for mass fractionation, so that a mass discrimination correction is not required. For the lighter elements, silver and copper, the mass discrimination correction does exceed the external error, and was applied to each sample. Both the daily and long-term external error have sufficient precision for concentration determinations, and so are suitable for this study. Indeed the suitability of this ICP-MS method for trace metal determination is limited by the blank values rather than the ratio precision. 
Table 2.7: Precision and accuracy for analysis of CASS-4 certified coastal seawater

\begin{tabular}{|c|c|c|c|c|}
\hline Date & $\mathrm{n}$ & $\mathrm{Ag}(\mathrm{pmol} / \mathrm{kg})$ & $\mathrm{Pb}(\mathrm{pmol} / \mathrm{kg})$ & $\mathrm{Cu}(\mathrm{nmol} / \mathrm{kg})$ \\
\hline $1-$ Oct -02 & 5 & $54 \pm 6$ & Not measured & $12 \pm 2$ \\
\hline $14-\mathrm{Feb}-03$ & 4 & $58 \pm 5$ & Not measured & contaminated** \\
\hline 12-Mar-03 & 5 & $52 \pm 7$ & $<\mathrm{LOD}$ & contaminated $^{* *}$ \\
\hline 23-Apr-03 & 4 & $60 \pm 3$ & $<$ LOD & $9.0 \pm 0.2$ \\
\hline 14-Jan-04 & 3 & $52 \pm 8$ & $64 \pm 7$ & $9.8 \pm 0.3$ \\
\hline 19-Mar-04 & 3 & $57 \pm 7$ & $111 \pm 13$ & $9.9 \pm 0.2$ \\
\hline 2-Jun-04 & 2 & $59 \pm 10$ & $55 \pm 7$ & $9 \pm 0.8$ \\
\hline 17-Aug-04 & 3 & $61 \pm 13$ & $76 \pm 10$ & $8.1 \pm 0.8$ \\
\hline 2-Nov-04 & 3 & $54 \pm 2$ & $72 \pm 10$ & $9.0 \pm 0.2$ \\
\hline Certified Value & & $56.6 \pm 8 *$ & $46 \pm 17$ & $9.1 \pm 0.9$ \\
\hline
\end{tabular}

\subsubsection{Accuracy and Precision of the Co-Precipitation Method}

To assess the accuracy of the APDC-IDMS procedure for porewaters the CASS-4, certified nearshore seawater produced by the National Research Council of Canada was analyzed normally in at least triplicate on each day. 5-10 mls of the seawater was extracted per sample to ensure adequate sensitivity to quantitate accurately. The results are shown in Table 2.7. The silver values agreed within 1 standard deviation to the certified value for each analysis day over two years of analysis. The copper concentrations also agreed with the certified value once a source of contamination was identified. The lead levels in the certified seawater are 40 times lower than expected in porewaters. On two occasions the measured concentration of lead in the standard seawater was higher than the certified value. On the other days the measured lead concentrations agreed within error with the certified value, though all measured values fall on the high side of the certified estimate. In order to get lead values with a higher precision and accuracy more seawater needs to be extracted to increase the signal to noise ratio. However it was decided not to do this as extracting more than $10 \mathrm{mls}$ of seawater would not be representative of the porewater method in which just. 1 to $2 \mathrm{ml}$ of sample is extracted.

To assess the long-term accuracy of a porewater analysis a large volume of porewater was collected from Buzzards Bay in March 2003. This porewater sample was used as an internal reference material and run at regular intervals. The results are given in Table 2.8. All three elements report concentrations that agree within error (Student's t-test $95 \%$ confidence 
Table 2.8: Measured concentration of a the Buzzards Bay porewater internal reference solution over time with a comparison of the precision reported by Rivera-Duarte and Flegal [1996] for their porewater internal reference material.

\begin{tabular}{|c|c|c|c|c|c|c|c|c|c|c|}
\hline \multicolumn{3}{|c|}{ Concentration (nmol $/ \mathrm{kg}$ ) } & \multicolumn{2}{|l|}{ Silver } & \multicolumn{3}{|c|}{ Lead } & \multicolumn{3}{|c|}{ Copper } \\
\hline Date & $\mathbf{n}$ & Mean & $s^{1}$ & $\% \mathrm{CV}$ & Mean & $s$ & $\% \mathrm{CV}$ & Mean & $s$ & $\% \mathrm{CV}$ \\
\hline 19-Sep-03 & 3 & 0.067 & 0.006 & 9.5 & 1.8 & 0.3 & 16 & 7.7 & 0.7 & 9 \\
\hline 9-Mar-04 & 3 & 0.065 & 0.0004 & 1 & 1.7 & 0.06 & 3 & 8.4 & 0.7 & 8 \\
\hline 2-Jun-04 & 3 & 0.072 & 0.004 & 6 & 1.9 & 0.1 & 8 & 7.4 & 0.1 & 1 \\
\hline 22-Sep-04 & 3 & 0.068 & 0.003 & 4 & 1.7 & 0.06 & 4 & 7.3 & 0.3 & 5 \\
\hline \multicolumn{11}{|c|}{ Rivera-Duarte and Flegal [1996] } \\
\hline & 3 & 0.2 & 0.02 & 8 & 14 & 1 & 8 & 65 & 7 & 11 \\
\hline
\end{tabular}

${ }^{1} s=$ standard deviation

interval) over the course of a year and have levels of precision that are better than those reported by Rivera-Duarte and Flegal [1996] for the extraction of $2 \mathrm{mls}$ of a porewater reference material with concentrations at least a factor of 5 times higher than those in the Buzzards Bay reference porewater. Rivera-Duarte and Flegal [1996] also analyzed for Cd, $\mathrm{Co}, \mathrm{Fe}, \mathrm{Ni}$ and $\mathrm{Zn}$ so the methods cannot be directly compared. However the Co-APDC method certainly enables more precise and equally accurate analyses of lead, silver and copper in small volumes of porewaters than have been reported previously.

\subsubsection{Verification of the Co-APDC Method for Porewaters}

The precision of the porewater APDC method is acceptable for porewater analysis and measurement of the standard seawater sample has indicated that the method is accurate in seawater. However it is important to verify that the complex porewater matrix does not introduce analysis artifacts that may degrade the accuracy of the analysis in porewaters. Due to the potentially high concentration of strong ligands in porewaters [Skrabal et al, 2000 ] it is possible that the Co-APDC does not completely scavenge all of the metal from solution as it precipitates. All of the porewaters were stored at $\mathrm{pH}<1$ with nitric acid for at least one month prior to analysis, so that some refractory organic matter will have decomposed, reducing this potential problem [Rivera-Duarte and Flegal, 1996]. The reduction in extraction efficiency due to strong ligands is not a problem as long as sufficient material is still recovered for analysis and if the isotope spike, which acts as an internal standard, has equilibrated with the sample. If the ligand is sufficiently strong, or kinetically inert 
Table 2.9: Comparison of untreated and ultra-violet light treated samples from the Hingham Bay and Massachusetts Bay sampling sites.

\begin{tabular}{rcccccccc}
\hline & Depth & $\mathrm{n}$ & \multicolumn{3}{c}{$\mathrm{Ag}(\mathrm{nmol} / \mathrm{kg})$} & \multicolumn{3}{c}{$\mathrm{Cu}(\mathrm{nmol} / \mathrm{kg})$} \\
& $(\mathrm{cm})$ & & Untreated & $s$ & UV treated & Untreated & $s$ & UV treated \\
\hline HB Sept 2002 & $0-2$ & 3 & 0.27 & 0.02 & 0.22 & 9.8 & 0.9 & 10.7 \\
Core 3 & $15-20$ & 3 & 0.42 & 0.06 & 0.49 & 26 & 2 & 23 \\
MB Feb 2002 & $0-3$ & 1 & 0.63 & & 0.62 & 75 & & 82 \\
Core 3 & $4-9$ & 1 & 0.24 & & 0.19 & 40 & 38 \\
& $11-28$ & 1 & 0.21 & & 0.2 & 29 & 41 \\
\hline
\end{tabular}

compounds are present, so that the bound metal portion does not equilibrate with the entire metal population then an extraction will underestimate the metal concentration. To assess if strong ligands are interfering with the analysis porewaters from a range of depths from both sampling sites were sub-sampled and half the sample was subjected to 5 hours of ultra-violet light (Ace Glass Inc, $1200 \mathrm{~W} \mathrm{Hg}$ vapor bulb) in the Moffett Laboratory. The UV light destroys the organic matter, so that any bound metals would be released. Both UV and untreated porewaters were then extracted by the Co-APDC method. The results are presented in Table 2.9. The Hingham Bay UV-treated samples all fall within the $95 \%$ confidence interval of the mean of the three untreated analyses according to Student's t-test. For Massachusetts Bay insufficient sample was available to do replicate analyses so that single analyses need to be compared. Taking a representative uncertainty in the analysis for the day (based on 3 Hingham Bay porewaters) of $6 \%$ for silver and $10 \%$ for $\mathrm{Cu}$ and applying Student's t-test there is no significant differences between the two treatments. Dissolved organic carbon thus does not interfere with the metal extraction processes. This is the case across the range of porewater depths which were measured.

\subsubsection{Standard Additions}

An additional difficulty associated with porewater analyses is that the metal concentration in porewaters could be at or close to saturation at many deptbs. Addition of the isotope spike may lead to the precipitation of an inorganic metal phase. If the metal phase that precipitates is not at the same isotope ratio as the sample and the extraction efficiency of the Co-APDC is poor then the measured isotope ratio will not be representative of the sample. To ensure that precipitation of a mineral phase was not occurring and to address any matrix effects associated with the digested Co-APDC precipitate, standard additions were added 

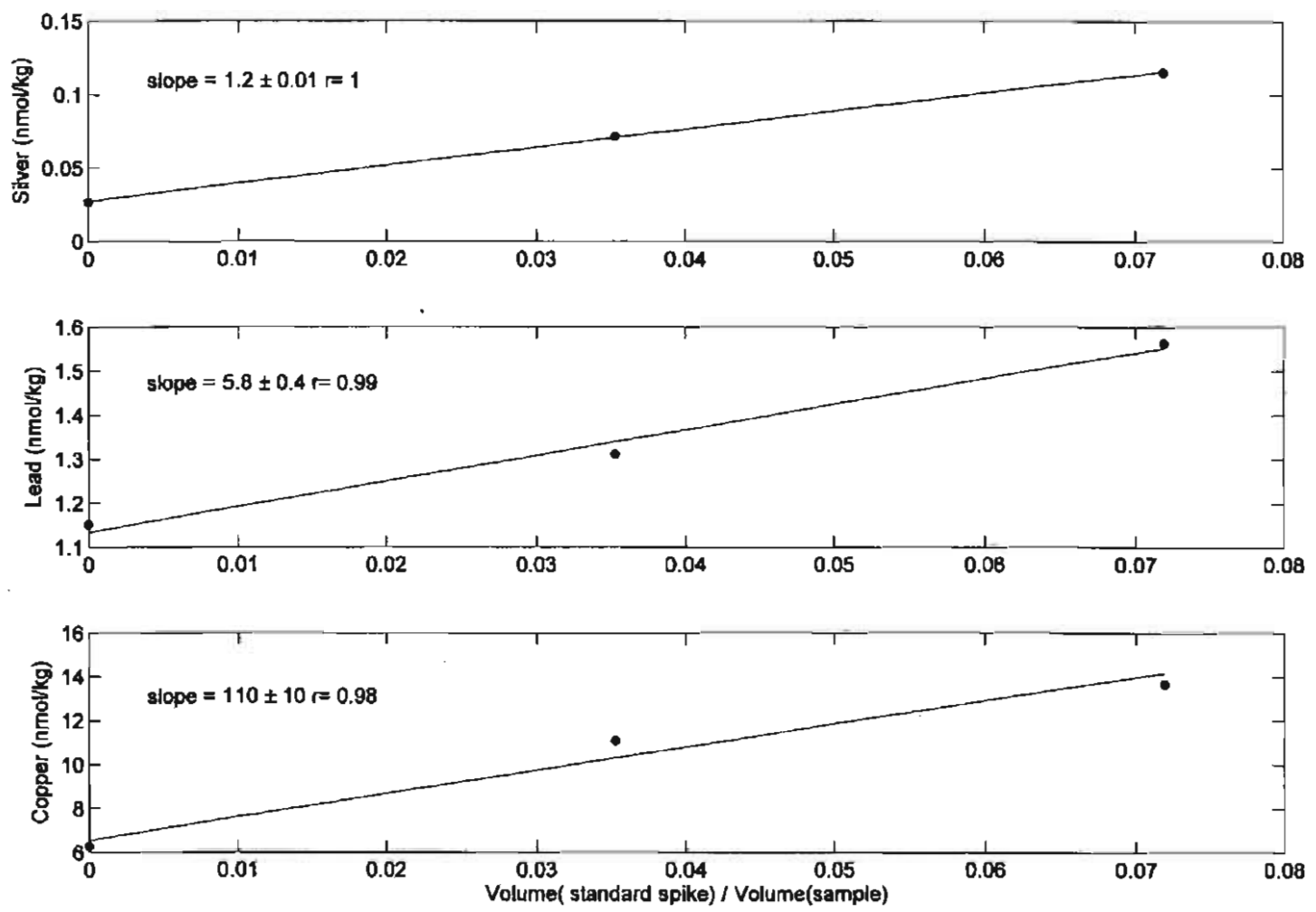

Figure 2-4: Example of a standard addition of a standard solution to a Hingham Bay porewater collected on June 2004 (Core $315-20 \mathrm{~cm}$ ). The standard concentration was $[\mathrm{Ag}]=1.3 \mathrm{nmol} / \mathrm{kg},[\mathrm{Pb}]=5.2 \mathrm{nmol} / \mathrm{kg},[\mathrm{Cu}]=106 \mathrm{nmol} / \mathrm{kg}$.

to porewater samples. Due to only a limited volume of sample being available, standard additions could not be performed on the majority of samples. Rather, larger samples of porewater that were collected to represent the different redox zones within the sediments at each site were assessed. At least one standard addition calibration curve was run during each ICP run. An example of the results for a standard addition analysis on Hingham Bay porewaters is shown in Figure 2-4. The linearity of the response was always good and the slope of the calibration curve agreed with that expected based on the concentration of the standard spike. This indicates that across the range of redox conditions at Massachusetts Bay and Hingham Bay the expected concentration in a spiked sample is correctly measured so giving confidence that there are no artifacts due to raising the metal concentration with the isotope spike and that any matrix effects are negligible compared to the precision of the isotope measurements. 


\subsection{Conclusions}

The co-precipitation method of Reuer and Boyle and Edmond [1977] has been modified for porewater analyses. A series of tests has determined that the method is not prone to analytical artifacts associated with the complex porewater matrix. The method has detection limits comparable to that developed by Rivera-Duarte and Flegal [1996] but due to the use of isotope dilution ICP-MS has better precision. The method is thus suitable for the determination of lead, silver and copper in porewaters. 


\title{
Chapter 3
}

\section{Seasonal Cycling of Silver, Lead}

\section{and Copper in Coastal Sediments}

\begin{abstract}
The role coastal sediments play in the biogeochemical cycles of many trace metals is poorly understood. For metals such as silver, copper and lead, this is especially important, as elevated concentrations are often found in coastal sediments due to human activities. In order to understand the diagenetic cycling and fate of these metals, porewater and solid phase profiles of silver, lead and copper at two contrasting coastal sites in Massachusetts, USA, have been collected. The first is a heavily contaminated site in Hingham Bay, Boston Harbor and the second site, in Massachusetts Bay, is a less impacted, offshore site. At both sites the trace metals are trapped by iron oxyhydroxides in oxic sediments. However, the two sites have very different redox conditions which leads to different trace metal cycles. The Hingham Bay site is a strongly reducing site, with free sulfide in the porewaters measurable within the top $7 \mathrm{~cm}$. The porewater profiles at this site indicate a strong seasonal cycle. Trace metals adsorbed to iron oxyhydroxides are released to the dissolved phase in spring, as the redox horizons shoal. Due to the presence of trace levels of sulfide above the anoxic zone, the dissolved trace metals are precipitated from the porewaters as insoluble sulfides. Porewater profiles collected in late summer do not show a release of trace metals associated with the reduction of iron oxyhydroxides, as in the highly reducing conditions that develop over spring and summer, most trace metals are transferred to the sulfide phase once their carrier iron oxyhydroxides are reduced. The trace metals are recycled back to the iron oxide phase over winter; the metal sulfides are oxidized as the redox horizons move downwards and bioturbation mixes reduced material upwards into the oxic zone. The trace metal cycle at Hingham Bay is thus an annual transfer back and forth between iron oxyhydroxides and sulfide phases. This is not the case in Massachusetts Bay. The porewater profiles show a co-incident release of trace metals with the reduction of iron oxyhydroxides throughout the year. Because the redox conditions at this site are less reducing than in Hingham Bay, the dissolved metals are preferentially re-scavenged by iron oxyhydroxides, rather than precipitated out as sulfides. This generates a solid phase enrichment of trace metals in the oxic surface sediments at this site. At both sites the surface $(0-3 \mathrm{~mm})$ porewater
\end{abstract}


copper concentrations are always greater than the bottom water concentration, suggesting a persistent diffusive flux of copper across the sediment-water interface. Only in winter is the silver porewater concentration high enough for there to be a silver flux across the interface. Lead is strongly scavenged and maintained at low concentrations in the surface porewaters throughout the year and so there is never a diffusive release of lead to the overlying waters. The diffusive flux of copper is calculated to be about $20 \%$ of the riverine copper input to Boston Harbor and thus an important term to understand in the copper budget for Boston Harbor.

\subsection{Introduction}

The role coastal sediments play in the biogeochemical cycles of many trace metals is poorly understood. It has been estimated that $90-95 \%$ of the trace elements that reach the ocean accumulate in coastal environments [Martin and Windom, 1991]. Particle reactive species, such as trace metals, accumulate in the sediments. Despite the large inventory of metals, it is not well established whether trace metals in coastal sediments are permanently sequestered or can be re-released to the overlying water column. Information on the extent of re-release from sediments is especially important for areas that are contaminated with metals due to human activity.

The sediments of the coastal zone have been a sink for contaminants discharged to the environment for decades. With improved environmental legislation, the release of pollutants and therefore the direct discharges of materials into the coastal zone have been significantly reduced [Bothner et al., 1998; Squire et al., 2002]. Now, however, the legacy of sediments contaminated with pollutants remains. Sediments have been shown to supply more lead [Rivera-Duarte and Flegal, 1994] and silver [Rivera-Duarte and Flegal, 1997a] to San Francisco Bay than riverine fluxes. Similarly in the north-eastern Irish Sea the benthic fluxes of copper and lead have been calculated to be of a similar magnitude to all river and direct waste inputs [Williams et al., 1998].

The benthic fluxes calculated in these studies are driven by the concentration gradient across the sediment-water interface. When the concentrations in the interstitial waters are greater than those in the water column, there is a flux out of the sediments. Diagenetic processes within the sediments determine the concentration of species in the porewaters, and so strongly influence the diffusive flux of material across the interface. Transition metal concentrations in porewaters are determined by the relative importance of supply and removal processes. Supply of trace metals to porewaters has been attributed to the 
release of metals from the early diagenetic degradation of organic matter |Klinkhammer, 1980; Heggie et al., 1986; Shaw et al., 1990; Fones et al., 2004]. Trace metals are often strongly scavenged by iron and manganese oxide phases [Vojak et al., 1985; Burdige, 1993; Aller, 1994; Wen et al., 1997] so these oxides can remove trace metals from porewaters. The reductive dissolution of these carrier phases can also release trace metals to porewaters [Shaw et al., 1990]. The formation of insoluble mineral phases, especially sulfides [Elderfield et al., 1981; Rosenthal et al., 1995] can remove trace metals from porewaters, while the oxidation of sulfides can release metals.

The sediments in the coastal zone are subject to strong seasonal variations in organic and terrigenous matter flux, water column stratification, temperature and benthic macroinvertebrate activity. The diagenetic processes and redox zones within the sediments can thus vary considerably over the course of a year. Because the porewater concentrations of metals are determined by so many competing processes, they are extremely sensitive to variations in the redox zones in the sediment. To fully understand the benthic flux of maetals from coastal sediments it is thus necessary to understand the seasonal variation in redox conditions as well as the important phases releasing or removing trace metals from the porewaters.

The aim of this study was to determine the fate of contaminant metals in the sediments by investigating the seasonal changes in redox conditions and the resultant changes in the trace metal cycling. High resolution porewater and solid phase profiles from two contrasting sites in coastal Massachusetts, USA were collected seasonally over two years. The redox environment was fully defined by comprehensive analysis of other species such as nutrients and sulfide. Three contaminant metals, silver, lead and copper (as well as iron and manganese) were investigated in detail so that a thorough understanding of the sediment geochemistry and the mechanisms leading to the release of these metals could be obtained.

\subsubsection{Metal Selection and Metal Chemistry}

Silver is frequently found at elevated concentrations in sediments due to releases from mining, smelting, electronic, dental and photographic industries [Ravizza and Bothner, 1996; Squire et al., 2002; Gobeil et al., 2005]. As many of these industries discharged their wastes directly into municipal sewers, levels of silver in sewage wastes up to 200 times the level in pristine marine sediments have been reported [Ravizza and Bothner, 1996]. As well as 
being a common metal contaminant, silver is a highly toxic metal to marine phytoplankton, zooplankton and small aquatic invertebrates, especially larval and embryonic stages [Ratte, 1999], even at trace levels. Particulate silver can also bioaccumulate in benthic organisms such as clams and polychaetes due to ingestion of contaminated sediments [Yoo et al., 2004].

Copper was selected for this study because many marine organisms are very sensitive to the concentration of copper. Copper is an essential micronutrient [Sunda, 1994] but at higher levels it can be toxic. For example elevated levels of copper have been demonstrated to influence phytoplankton species composition at levels of free $\mathrm{Cu}$ as low as $10^{-10} \mathrm{M}$, with the waters at this level toxic to cyanobacteria and some dinoflagellates [Moffett et al., 1997]. Sources of copper to the environment include electroplating, copper piping, photography, antifouling paints and pesticide formulations. Major industrial sources include mining, smelting, refining and coal-burning industries [Ward, 1995].

Lead was selected because the current global biogeochemical cycle of lead is dominated by human activity due to the use of lead additives in petroleum [Reuer and Weiss, 2002]. Other sources of lead to the environment include non-ferrous metal smelting, coal burning, pesticide and paint industries. The alteration of the lead biogeochemical cycle is detectable as elevated lead concentrations in the surface waters of the North Atlantic ocean. With the introduction of unleaded fuels the release of lead has been considerably reduced, and this is evident in the declining concentration of lead in the surface waters near Bermuda since the 1980's [Wu and Boyle, 1997].

To understand the fate and transport of anthropogenically released metals, their geochemistry in the natural environment must first be understood. Silver is a class B soft metal [Nieboer and Richardson, 1980]. It exists in the +1 oxidation state under environmental conditions. With 10 outer valence electrons it is a large easily polarizable cation that associates most strongly with sulfur and iodine containing ligands. Silver forms stronger complexes with reduced sulfur compounds than any metal, other than mercury, and is extremely particle reactive, second only to lead among commonly measured contaminant metals [Kramer et al., 2002]. The concentration of silver in natural surface waters is very low, in the pM range, and even highly impacted systems rarely exceed a few $\mathrm{nM}$ [Kramer et al., 2002]. Chloride is the dominant inorganic ligand of silver in marine waters; however, if trace amounts of reduced sulfur exist in oxygenated water [Rozan et al., 2000a] the complexation with these species would be expected to dominate the silver speciation [Kramer 
et al., 2002]. In the oceans silver behaves as a nutrient type element [Martin et al., 1983; Flegal et al., 1995; Ndung'u et al., 2001; Zhang et al., 2001; Ranville and Flegal, 2005] with low concentrations in surface waters $(0.25-12 \mathrm{pM})$ and regeneration at depth. The deep water concentrations in the South Atlantic (10-32 pM) are less than those in the North Pacific (43 - $86 \mathrm{pM}$ ) consistent with the ocean conveyor belt model of ocean circulation. The profiles of silver strongly correlate with dissolved silica [Martin et al., 1983; Flegal et al., 1995; Ndung'u et al., 2001; Zhang et al., 2001; Ranville and Flegal, 2005] and with dissolved copper [Martin et al., 1983; Ranville and Flegal, 2005]. Although silver has no known biological function $[X u$ and $W a n g, 2004]$ it seems that silver must be adsorbed onto the surfaces of particulate matter or taken up by or adsorbed to phytoplankton [Zhang et al., 2001\} in the surface oceans and then released at depth when these solids are dissolved or the organic matter is remineralized. There is evidence in the North Pacific [Zhang et al., 2001; Ranville and Flegal, 2005] and the Atlantic [Ndung'u et al., 2001] that surface waters exposed to prevailing winds from mainland regions have elevated silver concentrations. This is attributed to mineral and industrial aerosols, with the industrial inputs associated with high temperature coal burning and metal refining. The levels of enrichment in the surface Pacific Ocean are up to 50 times higher than background levels, so have led Ranville and Flegal [2005] to suggest that silver may be one of the most (if not the most) contaminated elements in the World Ocean.

Lead is a B-type metal, forming $\mathrm{Pb}$ (II) under environmental conditions, but is classified as an intermediate metal on the hard-soft metal scale Stumm and Morgan [1981]. Although lead is in the intermediate category, under the classification scheme proposed by Nieboer and Richardson [1980] its characteristics fall close to the soft metal boundary. Lead is thus a borderline soft metal and so associates strongly with the same ligands as silver. However, due to its intermediate behavior it also forms more complexes with ligands that associate with class A metals, namely ligands with oxygen and nitrogen donor atoms, than silver. In the oceans up to $50-70 \%$ of the dissolved lead is organically complexed [Shen and Boyle, 1987]. In coastal waters the complexation of lead is also dominated by organic ligands in surface waters, with complexation by sulfide species the most important in anoxic and suboxic waters [Rozan et al., 2003]. Lead is more particle reactive than silver [Kramer et al., 2002] and also found in $\mathrm{pM}$ to low $\mathrm{nM}$ concentrations in natural waters [Rivera-Duarte and Flegal, 1994; Rozan et al., 2003]. Lead has a scavenged element profile in the oceans 
[Bruland, 1983], with higher concentrations in surface waters due to atmospheric deposition and a decrease with depth due to particle scavenging.

Copper can exist in two oxidation states under the conditions encountered in the environment, copper (I) and copper (II). Copper (I) is a soft class B metal and is unstable in oxic seawater. Copper (II) is the thermodynamically stable form in seawater and forms complexes with ligands that associate with both class B metals (sulfur, iodine) as well as ligands that associate with class A metals.. In coastal waters, dissolved copper concentrations range from 2-150 $\mathrm{nM}$ and the copper is strongly complexed by organic ligands [Moffett et al., 1997]. Possible sources of ligands include cyanobacteria, likely as a detoxification mechanism in response to high dissolved copper concentrations [Moffett et al., 1997], terrestrial humic substances [Kogut and Voelker, 2001] and sediments [Skrabal et al., 1997; Shank et al., 2004]. In the open ocean, copper profiles fall into both scavenged and nutrient type behavior [Bruland, 1983] with surface depletion and a linear increase in concentration with depth. This mixed profile is maintained due to surface input of copper and removal in the upper waters with regeneration at depth. The linear nature of the profile is due to a bottom source of copper, indicating that the release of copper from sediments is important in the global biogeochemical cycle of copper.

\subsubsection{Study Area}

The study sites are located in coastal Massachusetts east of the city of Boston (Figure 3-1). The first site is in Hingham Bay in Boston Harbor and the second site is $9 \mathrm{~km}$ offshore in Massachusetts Bay. Both sites are fine-grained sediment sites in depositional areas [Knebel et al., 1991] of the Bay. The Hingham Bay site (Bothner et al. [1998] site number 8) is in $5 \mathrm{~m}$ of water and is representative of highly contaminated coastal sites as it is located $2.6 \mathrm{~km}$ to the northeast of the Nut Island sewage treatment plant which discharged solid sewage sludge to the Harbor until 1991. Boston Harbor was considered one of the most contaminated Harbors in the United States [Bothner et al., 1998] with the largest source of contaminants being sewage effluent discharged since the 1800's [Alber and Chan, 1994; Bothner et al., 1998]. A comprehensive source reduction program was undertaken to improve the water quality in Boston Harbor, and the key source reduction project was ending the discharge of sewage and sludge to the Harbor. This was facilitated by the construction of the new secondary treatment sewage plant at Deer Island and the discharge of sewage effluent 


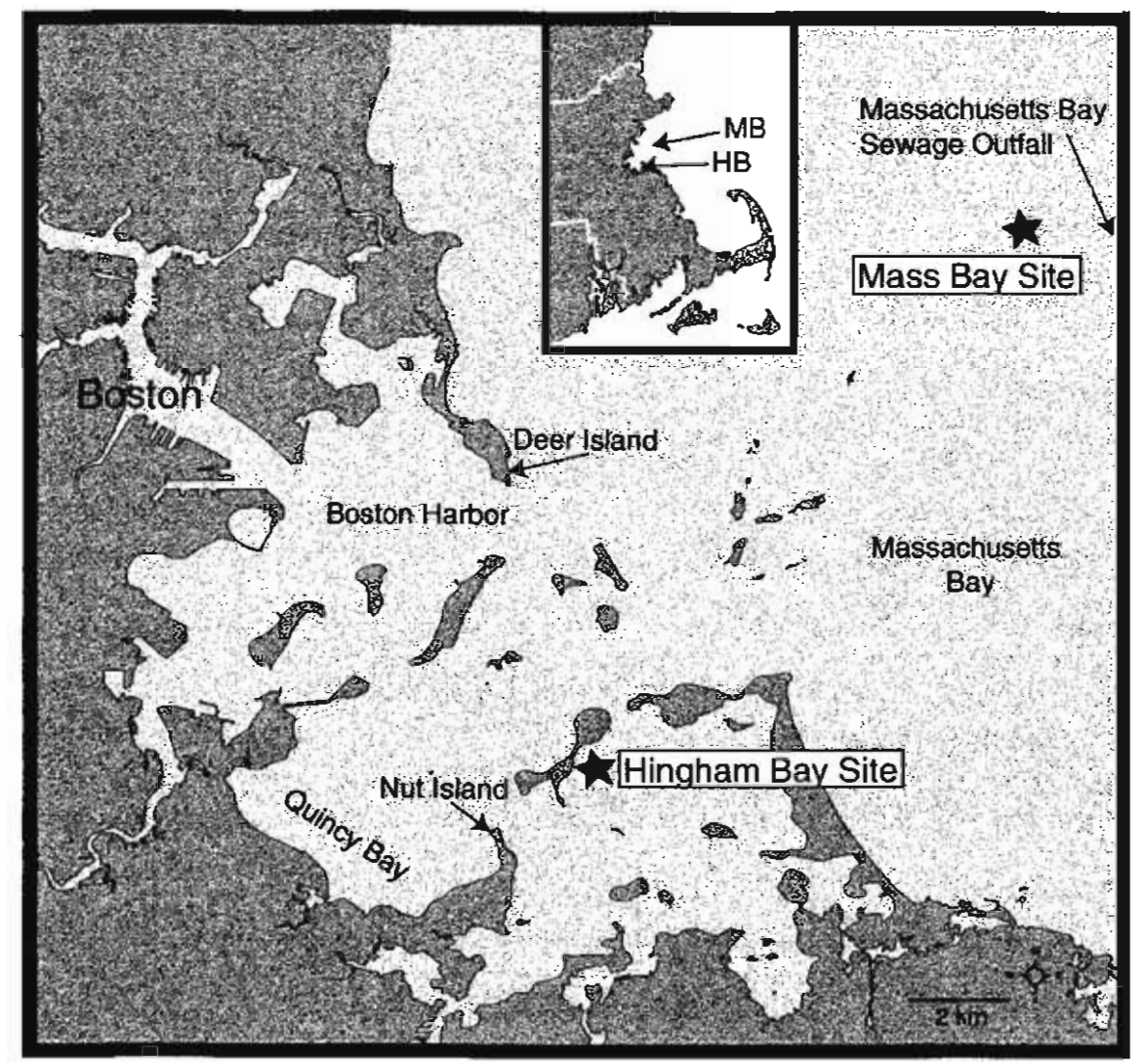

Figure 3-1: Map of coastal Massachusetts showing sampling site locations

in deeper offshore waters via an outfall tunnel. The outfall was opened in 2000. Due to the significant reduction in metal discharges, the concentrations of trace metals in the surface sediments of Boston Harbor have fallen over the timeframe 1977 - 1993. The concentration of both lead and silver in surface sediments has fallen by $50 \%$ at the Hingham Bay site [Bothner et al., 1998]. The second site, the Massachusetts Bay site (Bothner et al. [1998] site number 3 ) is in $30 \mathrm{~m}$ of water and is $2 \mathrm{~km}$ west of the newly constructed sewer outfall for Boston. The Massachusetts Bay site was selected by USGS to monitor the impacts of the new outfall tunnel. The physical characteristics of both sites are given in Table 3.1.

\subsection{Methods}

\subsubsection{Sample Collection}

Sediment samples from both sites were collected in a manner designed to minimize any disturbance to the sediment-water interface. Divers using SCUBA collected cores from the 
Table 3.1: Site Characteristics and Locations

\begin{tabular}{|c|c|c|c|c|c|}
\hline Name & Location & Water Depth & Collection Method & $\%$ sand $/$ silt $/$ clay $^{a}$ & $\% \mathrm{C}_{\mathrm{org}}{ }^{a}$ \\
\hline Massachusetts & $42.38981 \mathrm{~N}$ & $30 \mathrm{~m}$ & damped & $18 / 58 / 24$ & 2 \\
\hline Bay (MB) & $70.8305 \mathrm{~W}$ & & piston core & & \\
\hline Hingham & $42.29083 \mathrm{~N}$ & $5 \mathrm{~m}$ & diver & $6 / 62 / 32$ & 3 \\
\hline Bay (HB) & $70.92778 \mathrm{~W}$ & & collected & & \\
\hline
\end{tabular}

${ }^{a}$ Data courtesy M. Bothner USGS for $0-0.5 \mathrm{~cm}$ depth range

shallow Hingham Bay site, while at the Massachusetts Bay site a hydraulically damped piston corer [Bothner et al., 1998] was used. Core barrels were polycarbonate with a diameter of $10 \mathrm{~cm}$. At each site, collected cores were placed on ice immediately after collection and brought back to shore. Cores for porewater and solid phase analysis were sectioned under nitrogen as soon as logistically possible. For Hingham Bay samples the cores were driven back to WHOI and sectioned in a $4^{\circ} \mathrm{C}$ walk-in refrigerator, so that sectioning was complete within 10 hours of sample collection. In Massachusetts Bay samples were sectioned in a van at the dock in Boston at ambient air temperature and sectioning was completed within 8 hours of collection.

Sectioned sediments were centrifuged and the centrifuge vials returned to a nitrogen atmosphere where the porewater was carefully removed and filtered through a $0.45 \mu \mathrm{m}$ acrodisc filter. The filtered porewater was distributed into aliquots for analyses of trace metals, nutrients, total carbon dioxide, alkalinity, sulfide and sulfate. All sampling equipment in contact with samples for trace metals was acid cleaned, MQ soaked and dried in a laminar flow hood. The porewaters for trace metals were filtered directly into 4 ml vials pre-acidified with $40 \mu \mathrm{L}$ of trace metal grade (Fisher Optima) nitric acid, so that the porewaters were instantly preserved at a $\mathrm{pH}$ less than 1 . For the other analytes all sampling and storage equipment was. MQ soaked, rinsed and air dried in a laminar flow hood. More details on the sediment sectioning and sampling procedures are provided in Chapter 2. The dates on which each site was sampled are given in Table 3.2.

\subsubsection{Benthic Chambers}

Two benthic flux chambers were deployed during each cruise in Massachusetts Bay and on selected cruises in Hingham Bay (see Table 3.2). The benthic chambers are constructed of polycarbonate with a PVC lid. They enclose a sediment area of $345 \mathrm{~cm}^{2}$ along with about 3 
Table 3.2: Sampling Dates and Analyses Performed

\begin{tabular}{llccclll}
\hline Site & Date & No. of & Benthic & \multicolumn{2}{c}{ Porewaters Analyzed } & Solid Phase \\
& & Cores & Chamber & Ag,Cu,Pb & Fe & Mn & Analyzed \\
\hline Hingham & 16-Jan 2002 & 2 & yes & Core 1\&2 & Core 1\&2 & Core 2 & Core 2 \\
Bay & 8-Jul 2002 & 2 & no & Core 1\&2 & Core 1\&2 & Core 1 & Core 2 \\
& 26-Sept 2002 & 2 & yes & Core 1 & Core 1\&2 & & Core 2 \\
& 25-Oct 2003 & 1 & no & Core 1 & Core 1 & Core 1 & \\
& 16-Jun 2004 & 2 & yes & Core 1\&2 & Core 1\&2 & Core 1\&2 & \\
Mass. & & & & & & & \\
Bay & Oct 2001 & 3 & yes & & & & Core 1\&3 \\
& 7-Feb 2002 & 3 & yes & Core 1 & Core 1\&2 & & Core 3 \\
& 22-May 2002 & 2 & yes & Core 1 & Core 1\&2 & & \\
& 25-Oct 2002 & 2 & yes & Core 1 & Core 1\&2 & & \\
& 25-Sept 2003 & 2 & yes & & Core 2 & Core 1,2 & \\
\hline
\end{tabular}

$\mathrm{L}$ of overlying water, the precise volume of which is determined by bromide injection for each deployment. For most deployments, bottom water was circulated through gas permeable silicone tubing mounted within the chamber in order to prevent the oxygen level within the chamber from falling below $150 \mu \mathrm{M}$. The chambers were deployed for up to 5 days, and 8 water samples could be collected at preprogrammed times. All internal metal pieces on the chamber were replaced with plastic materials to make the chambers suitable for trace metal sampling. Samples were collected in aluminosilicate glass ampules. When the chambers were recovered, the ampules were stored in ice water and transported to Woods Hole. Samples for trace metals were $4 \mathrm{mls}$ in size and filtered with a $0.45 \mu \mathrm{m}$ acrodisc and acidified with trace metal grade nitric acid immediately upon removal from the ampules.

\subsubsection{Porewater and Solid Phase Analysis}

All metal analyses were performed under trace metal clean conditions in a clean room with overhead HEPA filtered air. Samples were only opened in a class 100 laminar flow hood. Wherever possible teflon plasticware was used for sample preparation. Teflon was soaked for at least 2 days in aqua regia followed by a leaching in boiling $50 \%$ nitric acid and rinsed with 18.2 M $\Omega$ Milli-Q (MQ) water. When teflon was not available HDPE was soaked for 4 days at $60^{\circ} \mathrm{C}$ in $2 \mathrm{~N} \mathrm{HCl}$ and rinsed 5 times with $\mathrm{MQ}$ water.

Acidified porewater samples were analyzed for iron and manganese with either a $\mathrm{Hi}$ tachi graphite furnace atomic adsorption spectrophotometer (GFAAS), quantified with a 
matrix-matched 5-point external calibration, or with a Finnigan Element 2 inductively coupled plasma-mass spectrometer (ICP-MS), operated in medium resolution with an indium internal standardization based on the method of Rodushkin and Ruth [1997].

Trace metal analysis of silver, lead and copper in porewater samples was undertaken on 1 to $2 \mathrm{mls}$ of acidified sample. A cobalt-ammonium pyrrolidine dithiocarbamate (Co-APDC) co-precipitation method was used to pre-concentrate the trace metals from the porewater matrix. The bottom water overlying the core was also analyzed with the Co-APDC method and 5 to $10 \mathrm{mls}$ of seawater was extracted. Isotope dilution ICP-MS was used to quantify the samples. Additional details on the Co-APDC method are provided in Chapter 2.

Porewater samples for nutrient analysis were determined on a Technicon autoanalyzer based on the method of Glibert and Loder [1977] with a detection limit of $0.5 \mu \mathrm{M}$ (pers comm. Joanne Goudreau). Sulfide was determined by the method of Cline [1969]; the detection limit was $1 \mu \mathrm{M}$. Sulfate was determined by ion chromatography. Alkalinity was determined by an automated Gran titration [Gieskes, 1973].

Alkalinity, total carbon dioxide $\left(\mathrm{TCO}_{2}\right)$, ammonium, phosphate, silicic acid and sulfide concentrations were used to calculate the $\mathrm{pH}$ of each porewater sample. The alkalinity contribution from dissolved organic matter in the porewaters was not taken into account due to lack of information. However, work done by Cai et al. [1998] shows that during estuarine mixing, the proton binding sites of humic substances become either completely protonated or deprotonated during estuarine mixing, and these sites do not contribute to alkalinity reactions. As the porewaters of both sites are at marine salinities the humic substances in porewaters are thus unlikely to have a large impact on the alkalinity. Additional experiments are required to investigate if other organic species in porewater may influence the alkalinity and thus pH. Salinity and temperature dependence of the acid dissociation constants for the carbonate system, nutrients, sulfide, water and boric acid were determined from the relationships given in Millero [1995]. For the first two years of this study alkalinity samples were analyzed within a week of sample collection. The alkalinity samples were not stored in an air-tight container and so over this time iron oxidation and precipitation would have occurred, thereby lowering the alkalinity. This was taken into account in the porewater $\mathrm{pH}$ calculation by assuming all of the iron measured in the porewaters precipitated. Due to the rapidity of iron precipitation [Roekens and Grieken, 1983] this is a good assumption. However it is unclear whether iron oxide dissolution occurred during the Gran titration and 
if a smaller correction is actually required. Unfortunately at a dissolved iron concentration of $200 \mu \mathrm{mol} / \mathrm{kg}$, there is a $\mathrm{pH}$ difference of 0.6 units between assuming no precipitation and $100 \%$ iron precipitation. This is a maximum uncertainty, as the concentration of iron in the porewaters is generally lower than this value. The precipitation of iron does not influence the $\mathrm{pH}$ values below $6 \mathrm{~cm}$, as the concentrations of iron below this depth are negligible. Further investigations are currently underway to quantify the impact of porewater iron oxidation on the measured alkalinity. For the moment the values of $\mathrm{pH}$ shown in Figures 3-30 to 3-38 are corrected to include $100 \%$ iron oxide precipitation.

The bicarbonate iron is responsible for between 82 and $96 \%$ of the porewater alkalinity. When iron concentrations are at their maximum, the precipitation of iron accounts for up to $15 \%$ of the alkalinity, and this is why iron precipitation has large impact on the $\mathrm{pH}$ values. Once all the iron is removed from the porewaters, the bisulfide iron is the second biggest contributor to the alkalinity, accounting for up to $6 \%$ of the total alkalinity.

The solid phases from the sectioned sediments were analyzed by the USGS with a total digestion method [Briggs and Meier, 1999]. The total digest method uses a series of acids to digest the sample with additions of concentrated hydrochloric acid, nitric acid, perchloric acid and hydrofluoric acid. Table 3.2 provides a list of all the metal analyses undertaken on each core.

Porosity and resistivity were determined for each cruise on an additional core collected at the same time. The porosity and resistivity data were required to determine the tortuosity of the sediments and to calculate benthic fluxes of solutes across the sediment-water interface. Resistivity was determined at a resolution of $0.062 \mathrm{~cm}$ with a probe with electrode spacing of $1 \mathrm{~mm}$ [Andrews and Bennett, 1981]. Two resistivity profiles per core were collected and following this the undamaged half of the core was sectioned for porosity. Porosity was determined gravimetrically by drying the sediments to a constant weight in a $60^{\circ} \mathrm{C}$ oven and assuming a grain density of $2.65 \mathrm{~g} \mathrm{ml}^{-1}$.

\subsection{Results and Discussion}

\subsubsection{Sediment Redox Conditions}

To investigate the metal cycling at each site it is essential to understand the redox conditions within the sediments. A discussion of the variations in the redox conditions in each season 
is thus provided below. Representative porewater profiles of nutrients and other analytes for each cruise are provided at the end of this chapter as this data is important for defining the redox conditions (see Figures 3-30to 3-34).

\subsubsection{Hingham Bay}

In Hingham Bay nitrate is consumed very close to the sediment-water interface in each season. Only in January (Figure 3-30) is nitrate removed in a smooth manner from a maximum at the interface to a concentration of zero by $1 \mathrm{~cm}$. In the other seasons the concentration of nitrate in the surface porewaters is negligible but there are small peaks in nitrate at erratic depths down to $5 \mathrm{~cm}$. These peaks have limited vertical extent and often consist of a single porewater sample with nitrate above the detection limit. These peaks in nitrate may be due benthic organisms irrigating the sediments with oxygenated waters.

The manganese concentrations observed range from $0-23 \mu \mathrm{M}$ and dissolved manganese is detectable in the uppermost porewater sample (representing 0-3 mm) in each season (blue diamonds in Figure 3-2). These concentrations are in the same range as reported by Elderfield et al. [1981] in Narragansett Bay but are considerably lower than those observed by Aller [1994] in Long Island Sound where concentrations reached up to $300 \mu \mathrm{M}$. The manganese concentration is higher in surface porewaters of Hingham Bay than in the overlying water, suggesting there is a flux of manganese to the water column throughout the year.

The manganese concentration reaches a maximum at a depth of $1 \mathrm{~cm}$ in January while in June and July the peak in Mn concentration is at the sediment-water interface (Figure 32). By October the subsurface peak has re-established at a depth of $0.6 \mathrm{~cm}$ with a steeper gradient in manganese concentration across the sediment-water interface than in January. A secondary peak in $\mathrm{Mn}$ concentration at $4 \mathrm{~cm}$ is evident in June, with the July and October profiles also showing less distinct maxima at the same depth. Below the maximum concentrations each profile shows a gradual decline, with the Mn concentration falling below the detection limits by between 9 and $13 \mathrm{~cm}$.

Dissolved iron is not detectable in the top $1 \mathrm{~cm}$ of porewater in January (red circles in Figure 3-2). Below this depth the Fe concentration rises, reaching a maximum at a depth around $2 \mathrm{~cm}$. In cores collected from June through October, there is a measurable concentration of iron in the surface porewaters and the peak in iron concentration occurs over a depth range of $0.5-4 \mathrm{~cm}$. By October there is no longer a measurable $\mathrm{Fe}$ concentration 

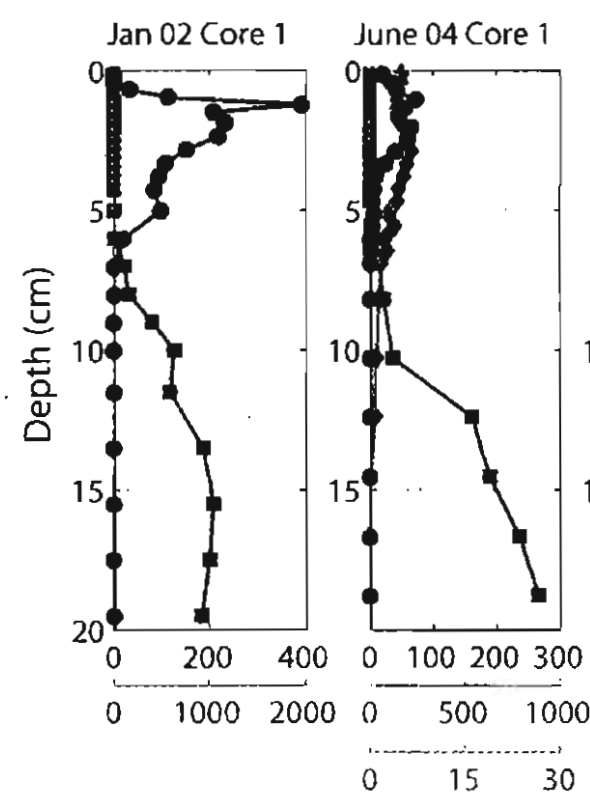

July 02 Core 1
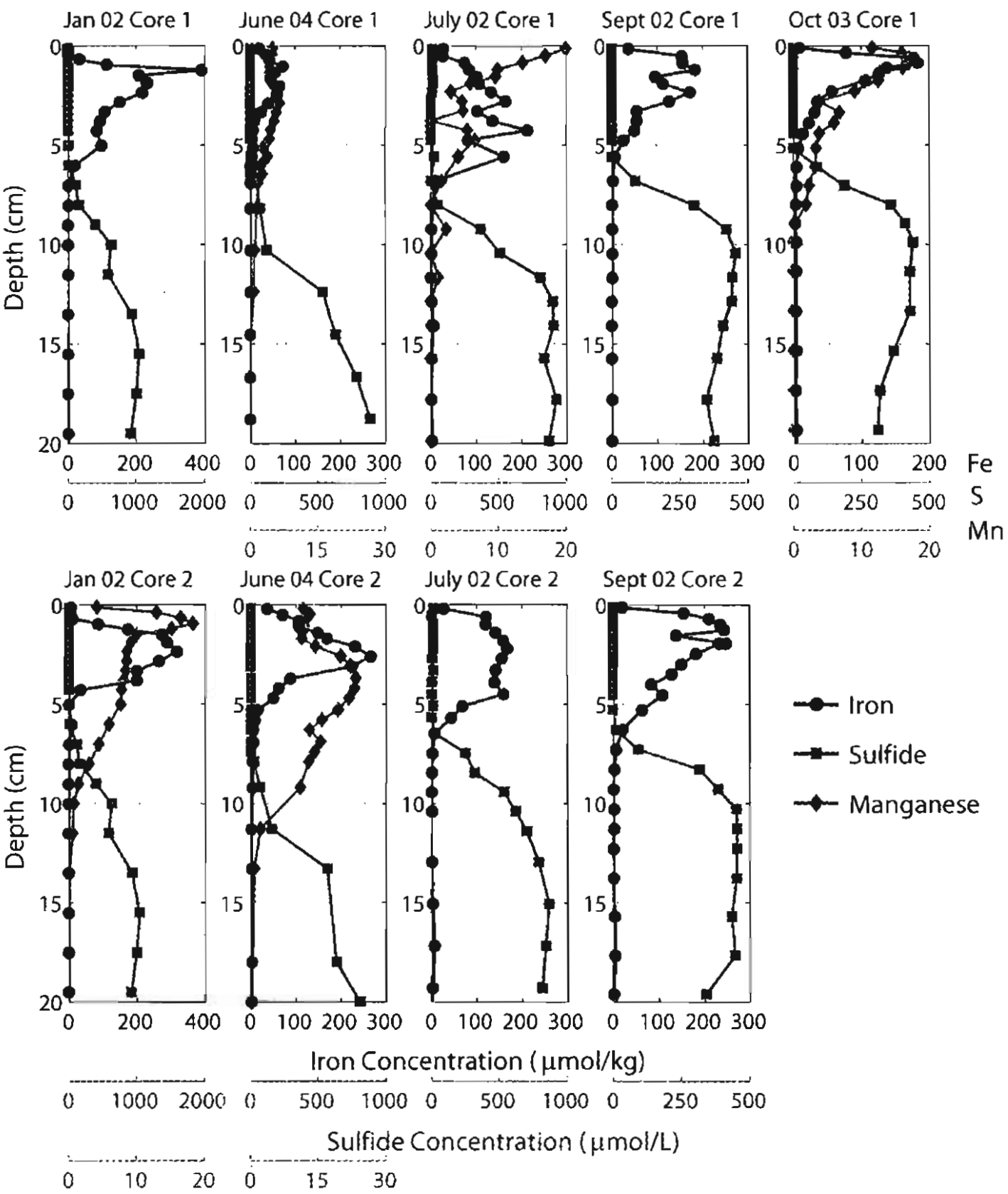

June 04 Core 2
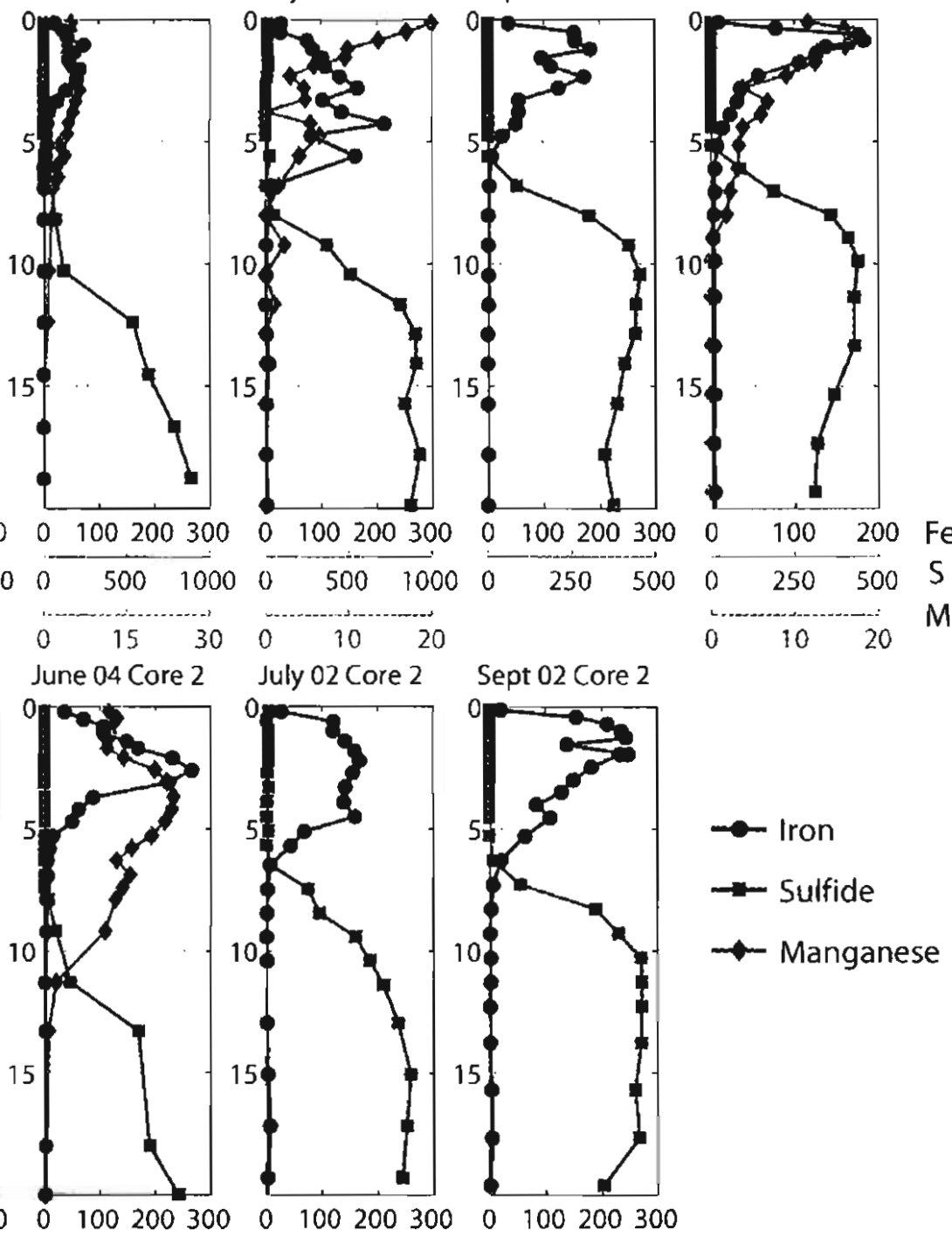

$$
\begin{aligned}
& \text { Iron Concentration }(\mu \mathrm{mol} / \mathrm{kg}) \\
& \text { Sulfide Concentration }(\mu \mathrm{mol} / \mathrm{L}) \\
& 500 \quad 1000 \text { ) } 500 \quad 1000 \quad 0 \quad 250 \quad 500 \\
& 15 \quad 30 \\
& \text { Manganese Concentration }(\mu \mathrm{mol} / \mathrm{kg})
\end{aligned}
$$

Figure 3-2: Iron, manganese and sulfide porewater concentration with depth at Hingham Bay for each cruise. The bottom water concentrations for iron are below the detection limit on the GFAAS, so are less than $0.5 \mu \mathrm{mol} / \mathrm{kg}$. The manganese bottom water concentrations range from $0.1-0.3 \mu \mathrm{mol} / \mathrm{kg}$. 
in the uppermost porewater sample, and the peak in Fe concentration occurs at $1.2 \mathrm{~cm}$. In all seasons, the Fe concentration declines more rapidly with depth than that of $\mathrm{Mn}$, with no detectable iron in the porewaters by a depth of $8 \mathrm{~cm}$.

The redox reaction sequence observed in pelagic sediments [Froelich et al., 1979] is also observed at this site, but with a compression of redox zones characteristic of organic rich coastal environments [Sorensen and Jorgensen, 1987]. This redox sequence is driven by heterotrophic microorganisms degrading organic matter as a source of energy and carbon [Morel and Hering, 1993]. The limited supply of oxidants generates biogeochemical zones within the sediments. Within these zones successive oxidants are consumed in the order of decreasing free energy production. There are no oxygen electrode measurements available, but oxygen must be completely consumed within millimeters of the sediment-water interface as nitrate is also consumed there. Due to the rapid exhaustion of the most energy yielding oxidants, manganese oxides are used as the next terminal electron acceptor, so that dissolved $\mathrm{Mn}(\mathrm{II})$ is released to the porewaters very close to the sediment-water interface.

In the January and July cores, the peak in iron concentration occurs when the curvature in the $\mathrm{Mn}$ profiles indicates that there is no longer a supply of $\mathrm{Mn}$ to the porewaters, consistent with the standard sequence of oxidants in which iron oxides are utilized as the terminal electron acceptor after the available labile manganese oxides have been consumed. In October and June, the depth zones in which there is release of manganese and iron to the porewaters overlaps. This is likely due to bioturbation and irrigation obscuring the diagenetic sequence when the profiles are close together.

Sulfate reduction yields less energy than the reduction of iron and manganese oxide phases, so that the release of sulfide to the porewaters at depths below $5-7 \mathrm{~cm}$ is consistent with the hierarchy of oxidant usage in early diagenesis [Froelich et al., 1979]. No sulfide is detected in shallow porewater samples and it is only measurable once the iron concentration falls below the detection limit. This indicates a tight coupling between iron and sulfide, with titration of sulfide by precipitation as insoluble iron sulfide phases:

$$
\mathrm{Fe}^{2+}+\mathrm{HS}^{-} \rightleftharpoons \mathrm{FeS}(\mathrm{s})+\mathrm{H}^{+}
$$

Only once all the available dissolved iron is consumed does the concentration of sulfide build up in the porewaters. Canfield [1989] found at a site in Long Island Sound (LIS) that even 
with active sulfate reduction the concentration of sulfide in porewaters was maintained at very low levels until all reactive iron was consumed. The porewater profiles of iron and sulfide at the LIS site are of the same form as in Hingham Bay, suggesting that likewise in Hingham Bay sulfate reduction is occurring through a much greater portion of the sediments than is indicated by the presence of sulfide. No iron or sulfide are detected simultaneously in Hingham Bay.

Alternative reaction pathways that are not directly linked with the oxidation of organic carbon may also be responsible for the observed order of reduced metal species released to the porewaters [Canfield et al, , 1993]. Iron(II) can be readily oxidized to iron(III) oxide by manganese (IV) oxides [Myers and Nealon, 1988; Aller, 1994; Thamdrup, 2000]. Thus the release of $\mathrm{Mn}$ to the porewaters may be a result of the abiotic oxidation of iron rather than due to dissimilatory $\mathrm{Mn}$ reduction. The oxidation of sulfide and sulfide mineral phases by both iron(III) and manganese(IV) oxides may also be important reaction pathways [Canfield, 1989; Burdige, 1993; Aller, 1994; Canfield et al., 1993; Thamdrup, 2000]. Porewater profiles may not definitively indicate the reaction mechanism but they do indicate where net production or loss of dissolved iron or manganese is occurring. This information is key for the investigation of trace metal cycling as both these oxides are known to be important scavengers of trace metals [Santschi et al., 1990].

The removal of iron from the porewaters at depth is most likely by precipitation of a sulfide phase while the removal of manganese is generally attributed to the formation of a manganese carbonate phase [Elderfield et al., 1981; Aller, 1994]. Manganese persists in the porewaters to a depth greater than that of iron. To determine the mineral phase responsible for the removal of manganese Figure 3-3 is a plot of total carbon dioxide concentration versus the manganese concentration. Following the example of Elderfield et al. [1981] the saturation boundary for rhodochrosite $\left(\mathrm{MnCO}_{3}\right)$ is also plotted for the range of $\mathrm{pH}$ observed in porewaters. The $\mathrm{pH} 7$ line is more representative of deep porewaters than the $\mathrm{pH} 8.5$ line. At all depths the samples are undersaturated with respect to the pure mineral phase. Elderfield et al. [1981] also found an undersaturation and attributed the removal of $\mathrm{Mn}$ to a mixed $\mathrm{Mn}, \mathrm{Ca}$ carbonate phase. There is a significant linear correlation between $\mathrm{Mn}$ and $\mathrm{TCO}_{2}$ (shown as the black line in Figure 3-3) with the relationship described by

$$
(0.54 \pm 0.06) \log [\mathrm{Mn}]+\log \left[\mathrm{TCO}_{2}\right]=(-5.1 \pm 0.1) \mathrm{r}=-0.83
$$




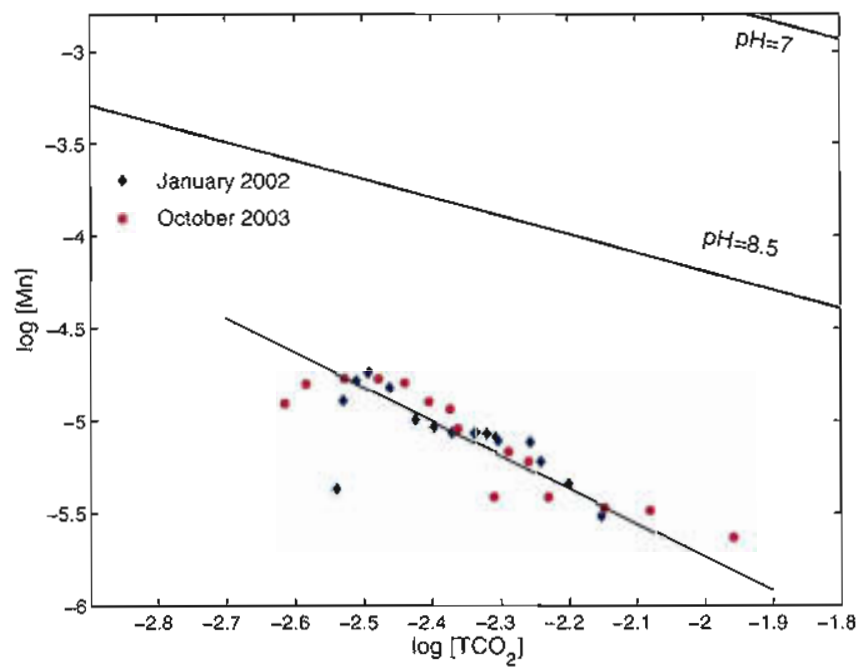

Figure 3-3: Logarithmic plot of total carbon dioxide concentrations $\left(\mathrm{TCO}_{2}\right)$ versus manganese concentration for January 2002 (Core 2) and October 2003 (Core 1). The saturation boundary for rhodochrosite $\left(\mathrm{MnCO}_{3}\right)$ for the $\mathrm{pH}$ values found in the porewaters is also shown. The black line is a linear regression of the January and October data. $\mathrm{K}_{s p}\left(\mathrm{MnCO}_{3}\right)=10^{-9.3}$ [Morel and Hering, 1993]; activity coefficients, $\gamma_{T M n^{2+}}=0.21$; $\gamma_{\mathrm{TCO}_{3}^{2-}}=0.021$ [Elderfield et al., 1981].

Following a rearrangement of the expression for the solubility product of $\mathrm{Mn}_{x} \mathrm{Ca}_{1-x} \mathrm{CO}_{3}$ Elderfield et al. [1981] showed that the slope of the above equation is equal to $x$, the mole fraction of $\mathrm{MnCO}_{3}$ in $\mathrm{CaCO}_{3}$. Thus at Hingham Bay $\mathrm{Mn}$ is removed by formation of a $\mathrm{Mn}_{0.54 \pm 0.06} \mathrm{Ca}_{0.46 \pm 0.06} \mathrm{CO}_{3}$ phase. This compares very closely with the $\mathrm{Mn}_{0.49} \mathrm{Ca}_{0.51} \mathrm{CO}_{3}$ phase identified by Elderfield, suggesting that this mixed $\mathrm{Mn}$, Ca carbonate phase is a commonly formed authigenic mineral phase in coastal sediments.

\section{Seasonal Cycle}

There is a distinct seasonal oscillation in the depths of the redox zones over the course of a year in the sediments of Hingham Bay. This site is shallow and flushed regularly by tidal currents (see Chapter 5) so that there is no seasonal stratification generating a depletion of oxygen in the bottom water. The seasonal cycle at this site is thus driven by the temperature and the flux of organic matter. In January, when the average water temperature in Boston Harbor is $4^{\circ} \mathrm{C}$ (NOAA), the surface sediments show a distinct $0.5 \mathrm{~cm}$ deep zone, with nitrate in the porewaters (Figure 3-30) and no measurable iron (Figure 3-2). From the curvature of the porewater profile manganese oxide reduction is occurring by $0.5 \mathrm{~cm}$ and iron oxide 
reduction by $1.5 \mathrm{~cm}$. In Boston Harbor the primary production in the overlying water rises steadily from very low levels in February to a maximum in productivity in summer, with the maximum usually occurring in July, but sometimes being as late as September [Keller et al., 2001]. Low reaction rates in the cold temperatures as well as limited supply of fresh organic matter due to the restricted phytoplankton growth reduce the demand for oxidants in winter, so that an oxic zone is established.

By late spring the water temperature has risen to $17^{\circ} \mathrm{C}$ and production in the water column is close to the highest recorded throughout the year. With the rain of fresh organic matter to the sediment-water interface due to the increased productivity, available oxidants are consumed at a faster rate and so are depleted closer to the sediment-water interface than in winter. This is evident in both the June and July profiles, with the manganese porewater profile indicating that manganese oxides are being reduced within the first porewater sample. The sediments are thus sub-oxic by the first $3 \mathrm{~mm}$ by late spring. There are no manganese data for September, but there is a steeper gradient in iron concentration through the top 6 $\mathrm{mm}$ in September than in July, indicating that the redox horizons have continued to move upwards over this time. By October the average temperature has cooled to $13^{\circ} \mathrm{C}$ from the annual maximum of $20^{\circ} \mathrm{C}$ recorded in August and the primary productivity has fallen from the summer high [Keller et al., 2001]. However, the demand for oxidants is obviously still high as the manganese and iron profiles are compressed tightly together at the sedimentwater interface. The iron concentration in the first porewater sample in October is $9 \mu \mathrm{M}$, having fallen from an average concentration of $28 \mu \mathrm{M}$ in September. This may be due to spatial variability but may also suggest that the remineralization rates, although still high, are starting to slow back down to the values observed in winter.

The depth at which sulfide first appears, the sulfide interface, also rises over the course of a year with the interface rising from $8 \mathrm{~cm}$ in June to $6 \mathrm{~cm}$ in October. By January the interface has moved back down, but the level is higher than that in June. This difference may due to variability between years or may indicate that there is a lag in the sulfide response to changes in the oxidant demand at the sediment-water interface. More data is required to confirm this.

Many of the iron profiles show sharp peaks that are unsustainable as steady state features. The multiple peaks in Core 1 of July 2002 (Figure 3-2) are not found in the replicate core. This indicates that these features are of limited spatial extent. The peaks are most 
likely due to the activity of benthic organisms bioturbating and irrigating the sediments. Flushing of axygenated water into sub-oxic burrows will remove iron from solution and generate peaks in the porewater profile. Studies on nearby sites in Massachusetts Bay [Wheatcroft et al., 1994] and Buzzards Bay [Martin and Sayles, 1987] have shown that bioturbation is greater in summer than in winter. Irrigation is also stronger in summer [Martin and Sayles, 1987], extending down to $20 \mathrm{~cm}$. Although the Hingham Bay site is much more sulfidic than either of these sites, and so cannot be directly compared, the incidence of the sharp peaks in the porewater profiles is also greater in the warmer months, supporting the notion that the peaks are generated by benthic organisms. The peaks are only evident in the iron profiles, and not the manganese profiles. Iron is much more sensitive to oxidation than manganese. The half life for $\mathrm{Fe}(\mathrm{II})$ in oxygen saturated seawater $\left(\mathrm{pO}_{2}=0.19-0.22\right)$ with respect to abiotic oxidation at $\mathrm{pH} 7$ is 30 minutes and at $\mathrm{pH} 7.9$ is 2.8 mins at $20^{\circ}$ and [Roekens and Grieken, 1983]. Although the presence of other porewater species such as phosphate and DOC will alter the oxidation rates, half-lives of less than half an hour imply that even a short-lived irrigation event that introduces oxygenated waters to sub-oxic regions will remove iron(II) from the porewaters. Manganese(II) oxidation is slower, with a half life of 500 yrs for abiotic Mn oxidation in seawater [Sunda and Huntsman, 1987]. This is not a relevant timescale as oxidation is enhanced by heterogeneous catalysis on mineral surfaces or can be bacterially mediated [Landing and Bruland, 1987], so the reaction rate will be faster in porewaters, but the far smoother Mn profile in July indicates that the timescale of $\mathrm{Mn}$ oxidation is slower than the timescale of the irrigation events.

The replicate cores collected on each cruise show differences in concentrations between cores but show approximately the same depths of the redox zones. Sediments are notoriously heterogeneous environments [Zhang et al., 2002; Fones et al., 1998, 2001] so the differences in concentrations are not surprising. Except for the June 2004 cores the differences in concentrations between replicates is largely due to the sharp peaks in the profiles, which as already discussed are probably non-steady state features driven by localized benthic organism activity. The June 2004 cores have the most marked differences between cores, with Core 1 reporting iron and manganese concentrations only $25 \%$ of the concentrations in Core 2 and lower than reported in any other core throughout the year, while the sulfide concentrations are similar. The reasons for this are unclear. The consistency of the redox zone depths between duplicate cores is encouraging as the redox zones will bave a strong 
impact on trace metal cycling.

\section{Solid Phase Data}

The relationship between the total digestion solid phase data and porewater data for Hingham Bay in February is shown in Figure 3-4. The available solid phase data from all other cores, together with the appropriate porewater data, are appended at the end of this Chapter in Figures 3-39 to 3-41. Figure 3-4 shows that in winter both iron and manganese solid phase data show a slight (10\%), surface enrichments in the upper $1 \mathrm{~cm}$ that are coincident with the depths of removal of the porewater phase towards the surface. These surface enrichments are due to the internal cycling of these metals. Surface enrichments of iron and manganese are observed in oceanic environments with less reducing sediments [Klinkhammer et al., 1982; Shaw et al., 1990] and a seasonal surface enrichment in manganese is observed in LIS [Aller, 1994]. Iron or manganese, released to the porewaters below the sediment-water interface due to the remineralization of organic matter, can diffuse or be mixed upwards, where it encounters oxidizing conditions. If oxidation and precipitation can occur faster than the timescale for diffusive escape from the sediments, the metals will be immobilized close to the sediment-water interface and an enriched surface layer will form. The solid phase iron data in the upper $10 \mathrm{~cm}$ over a year is shown in Figure 3-5. It can be seen that this surface enrichment does not persists throughout the year. The reduced oxic layer over the warmer months restricts internal cycling as the zone at the surface in which iron and manganese can be trapped is reduced, so that a flux to the overlying water is likely, as is evident by the measurable porewater concentrations of iron and manganese in the surface porewater samples in summer and fall.

\subsubsection{Massachusetts Bay}

The porewater iron profiles for the Massachusetts Bay site are shown in Figure 3-6 along with the available Mn data. Sulfide was measured on all these cores, but no sulfide above the detection limit of $1 \mu \mathrm{M}$ was recorded. The profiles of nutrients and other analytes for each cruise are provided at the end of the Chapter in Figures $3-35$ to $3-38$. The immediately obvious difference between the Massachusetts Bay site and the Hingham Bay site is that because there is no sulfide detected, dissolved iron persists down to much deeper levels, with $\mathrm{Fe}$ recorded below $30 \mathrm{~cm}$ in several cores. The nitrate profiles are characterized by 


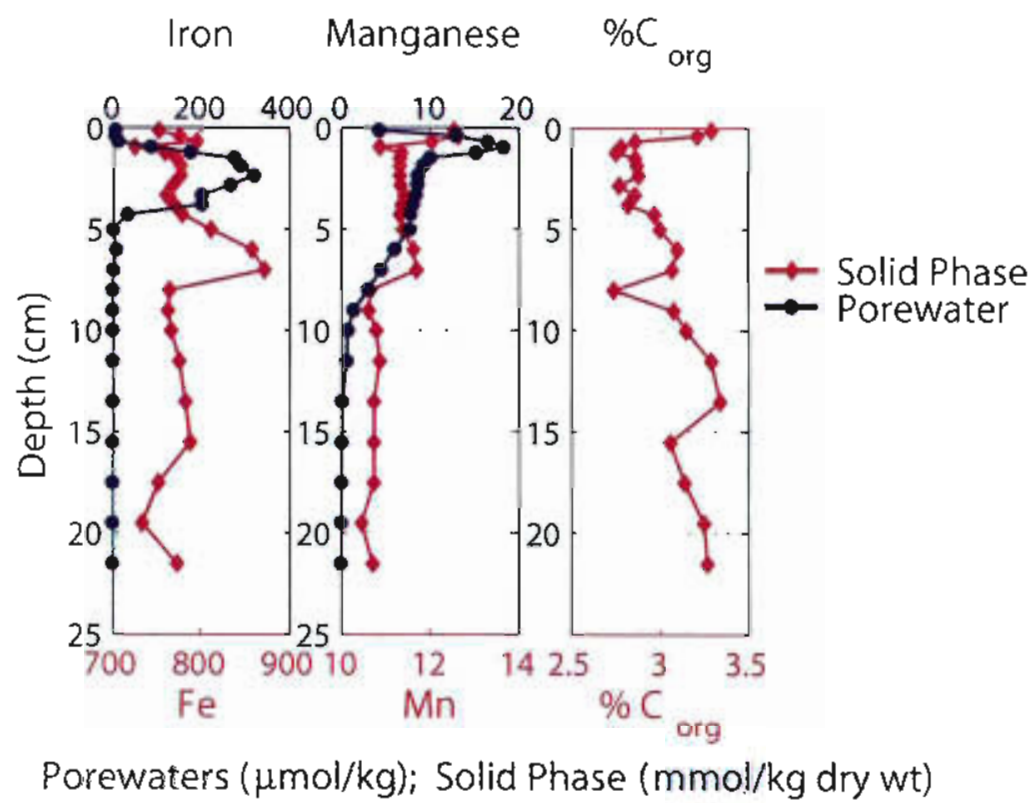

Figure 3-4: Solid phase iron, manganese and \% organic carbon profiles for Hingham Bay January 2002 (Core 2). The porewater profiles of iron and manganese are also shown.

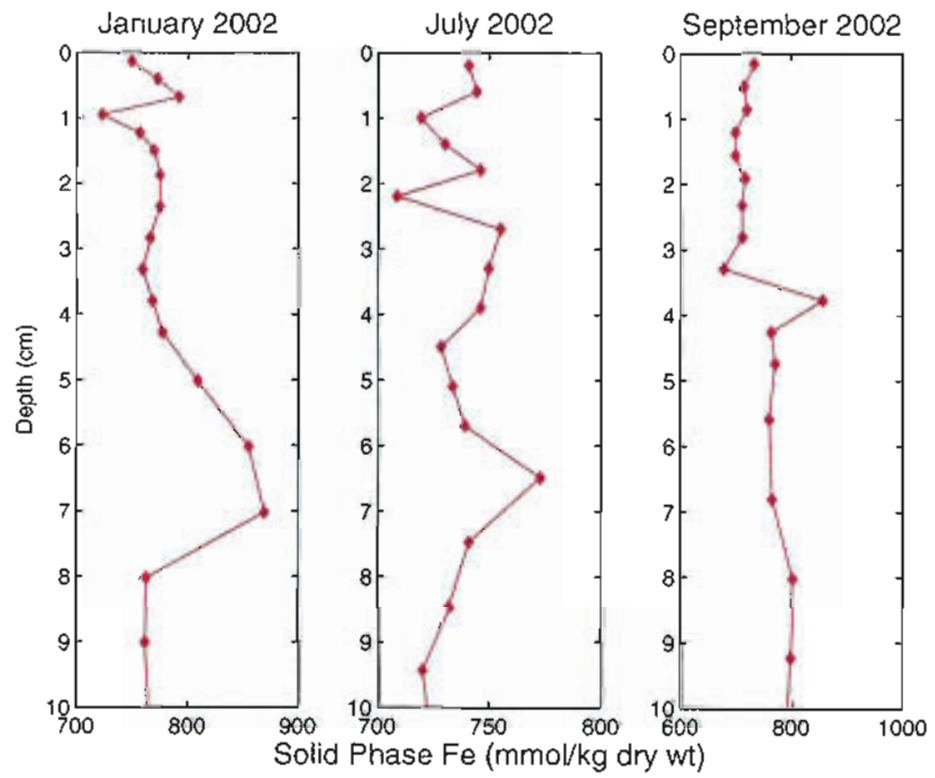

Figure 3-5: Solid phase data for the upper $10 \mathrm{~cm}$ for iron in January, July and September 2002. 

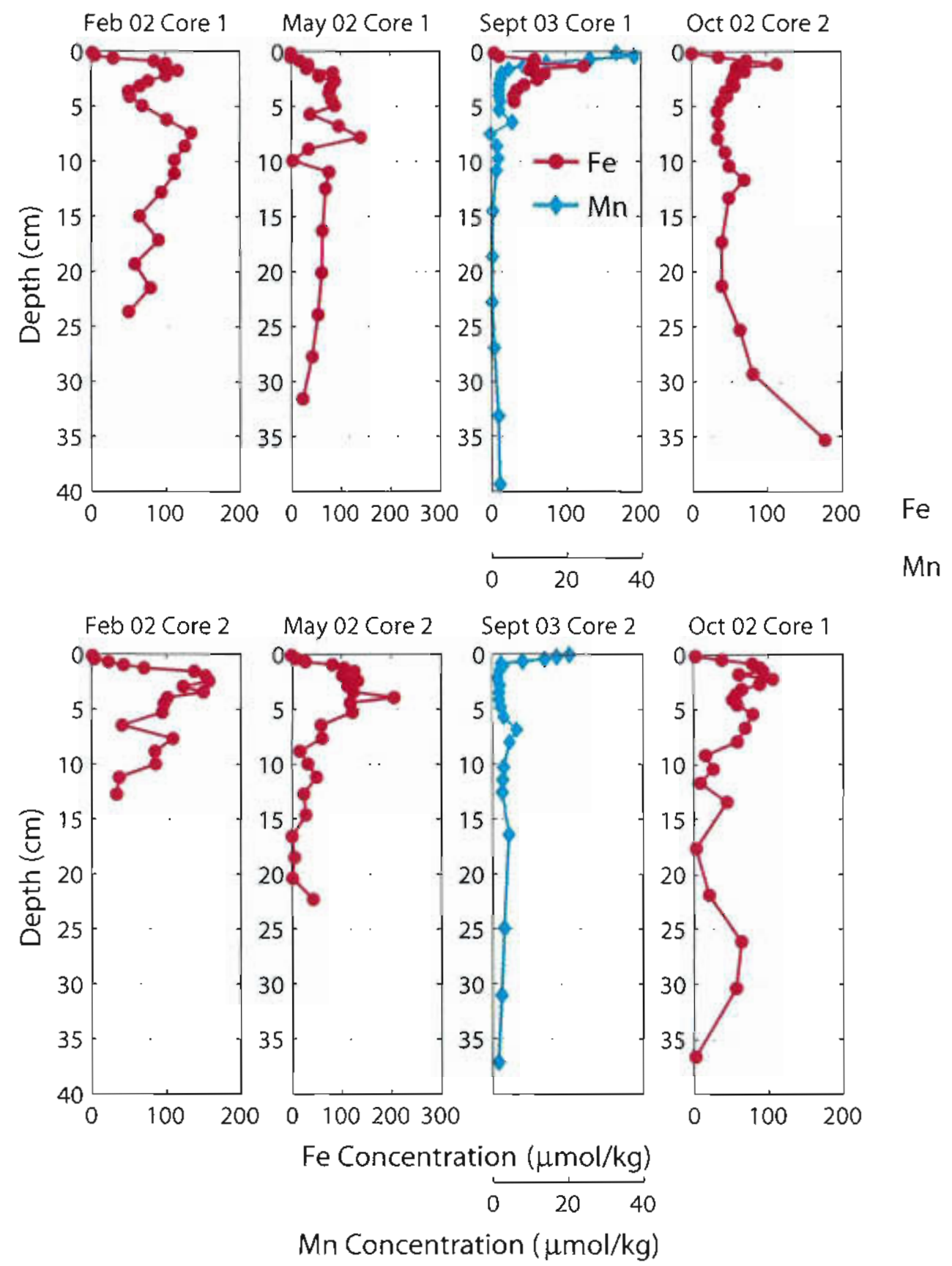

Figure 3-6: Iron porewater concentration with depth at Massachusetts Bay for each cruise. No sulfide was detected in any sample. Manganese data is also plotted where available. 
sharp spikes consisting of one or two samples with no nitrate detected in the remaining samples. The Massachusetts Bay site is considerably less reducing than the Hingham Bay site. From benthic chamber data the organic carbon oxidation rate at the Hingham Bay site was $1200 \mu \mathrm{M} \mathrm{C} \mathrm{cm}{ }^{-2} \mathrm{y}^{-1}$ while that for the Massachusetts Bay site was $600 \mu \mathrm{M} \mathrm{C}$ $\mathrm{cm}^{-2} \mathrm{y}^{-1}$ (pers. comm. W.R. Martin). This difference in organic carbon oxidation rate leads to considerable differences in the geochemical cycling at each site.

The maximum dissolved iron concentrations at the Massachusetts Bay site are less than those at Hingham Bay, and the profiles do not display a rapid removal at depth, consistent with the site being less reducing than Hingham Bay. Instead there is a shallow subsurface peak in all cores and additional peaks at depth. The locations of the second subsurface peaks are consistent between replicate cores, but the concentration and exact details vary slightly. The concentration of iron in the uppermost porewater samples is below the detection limit in all measured cores, suggesting that iron is internally recycled within the sediments. Porewater data are consistent with the removal of iron from the porewaters of these sulfide-free cores by the precipitation of vivianite $\mathrm{Fe}_{3}\left(\mathrm{PO}_{4}\right)_{2} \cdot 8 \mathrm{H}_{2} \mathrm{O}$. As shown by Figure 3-7 the ion activity product is very close to the $K_{s p}$ for this mineral phase below a depth of $1 \mathrm{~cm}$. Equilibrium with vivianite was also found by Martens et al. [1978] in Long Island Sound. Another possible mineral phase is siderite, $\mathrm{FeCO}_{3}$. However as shown in Figure 3-8 the iron concentration is generally undersaturated with respect to $\mathrm{FeCO}_{3}$ at all depths.

Manganese is released to the porewaters at a shallower depth than iron, as in Hingham Bay, and in September there is measurable concentration of $\mathrm{Mn}$ in the first porewater sample, so that a diffusive manganese flux to the overlying waters would be expected at this time. The manganese in the surface porewaters is higher than in Hingham Bay and the peak in $\mathrm{Mn}$ is removed very quickly with depth. The higher concentrations are likely due to the fact that more $\mathrm{Mn}$ is internally recycled at this site due to the less reducing conditions. More Mn porewater data from the cores collected in different seasons is needed to confirm this. $\mathrm{Mn}$ is never completely removed from the porewaters and the mechanism behind the rapid removal of the surface peak is unclear. Figure 3-9 investigates the relationship between Mn and $\mathrm{TCO}_{2}$. All samples are undersaturated with respect to a pure rhodochrosite mineral, phase but unlike in Hingham Bay, there is also no evident relationship with carbonate. The data in the figure fall into two distinct groups in both cores: the samples from within 


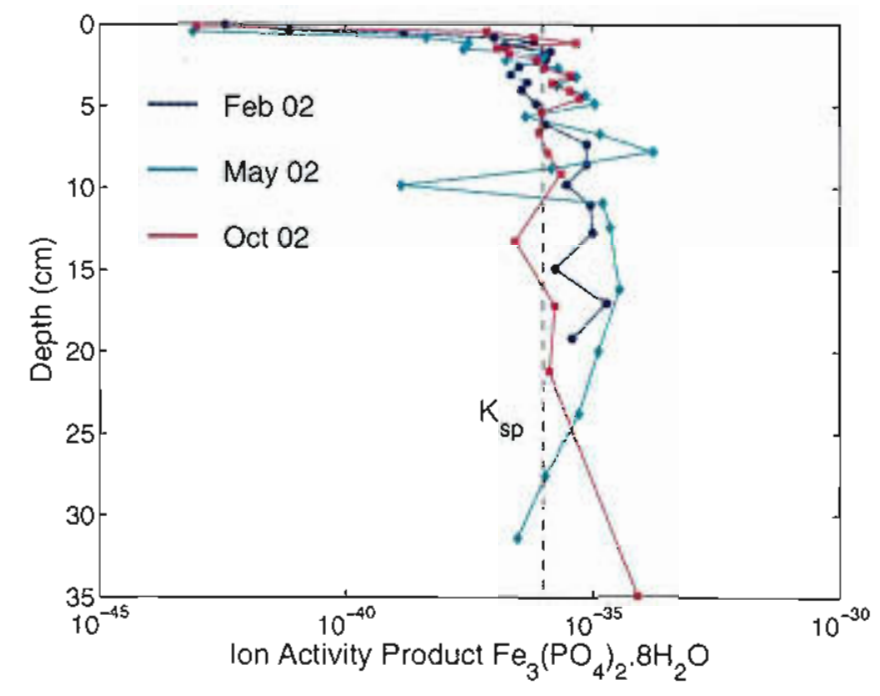

Figure 3.7: Plots of the ion activity product for vivianite $\mathrm{Fe}_{3}\left(\mathrm{PO}_{4}\right)_{2} .8 \mathrm{H}_{2} \mathrm{O} \mathrm{K} \mathrm{K}_{s p}=10^{-36.1}$ [Martens et al., 1978]. $\gamma_{T F e^{2+}}=0.0299$, phosphate concentrations from the empirical equations of [Millero, 1995].

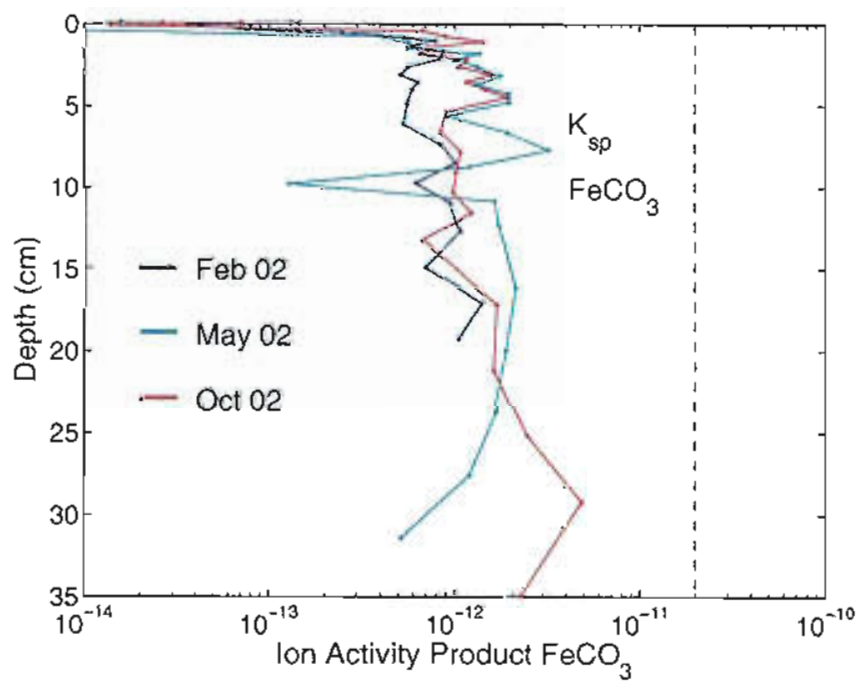

Figure 3-8: Plots of the ion activity product for $\mathrm{FeCO}_{3}$ as a function of depth at the Massachusetts Bay site. $\mathrm{K}_{s p}\left(\mathrm{FeCO}_{3}\right)=10^{-10.7}$ [Morel and Hering, 1993]. $\gamma_{T F e^{2+}}=0.0299$; $\gamma_{T_{C O}^{2-}}=0.021$ [Elderfield et al., 1981] [Morel and Hering, 1993]. 


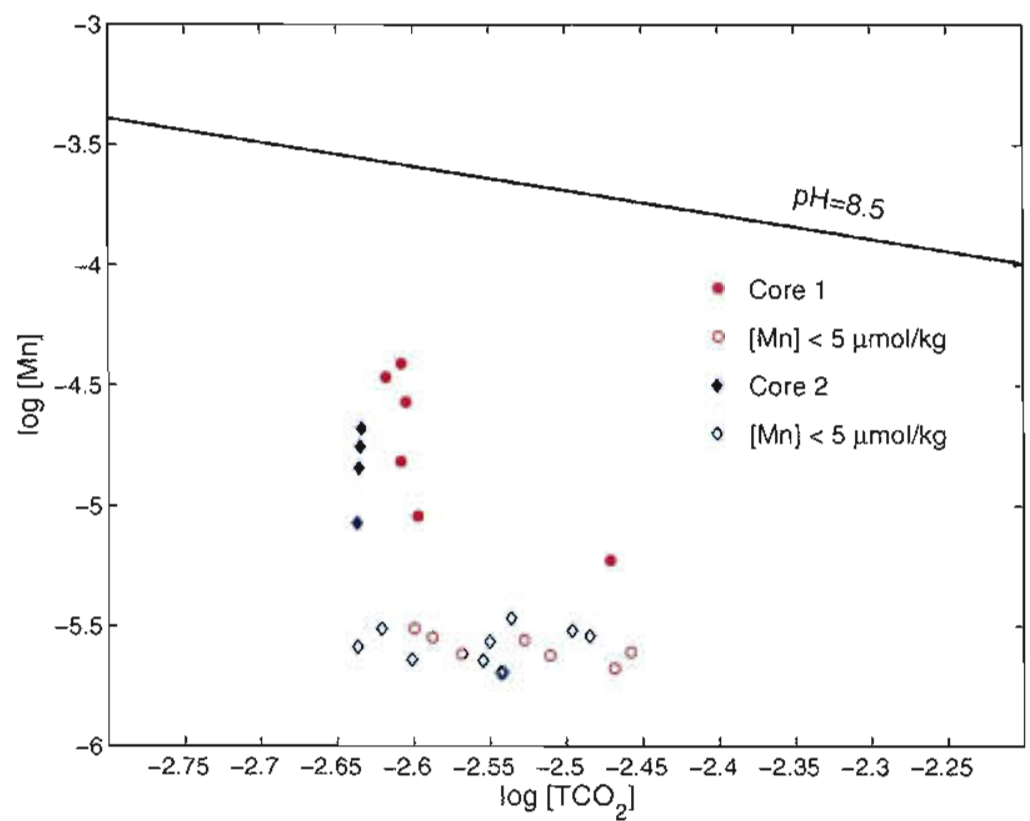

Figure 3-9: Logarithmic plot of total carbon dioxide concentrations $\left(\mathrm{TCO}_{2}\right)$ versus manganese concentration for September 2002 Core 1 and Core 2) and October 2003. The saturation boundary for rhodochrosite $\left(\mathrm{MnCO}_{3}\right)$ for the $\mathrm{pH}$ values found in the porewaters is also shown.

the $\mathrm{Mn}$ peak vary in concentration independently of carbonate, while the opposite is the case for the samples below the peak in which the Mn concentration remains just above the detection limit of $1 \mu \mathrm{M}$ and relatively constant despite large variations in $\mathrm{TCO}_{2}$. Some phase other than carbonate must thus be controlling the $\mathrm{Mn}$ removal. Mn has been shown to adsorb to sediment particles with a relationship between sediment grain size as well as Mn oxide content [Canfield et al., 1993]. If manganese oxide phases that are more refractory to early diagenetic processes persist at depth they may be controlling the $\mathrm{Mn}$ porewater concentration. Why this has not been observed at other sites, and a carbonate control is generally observed [Burdige, 1993] is unclear.

Only in Core 1 of May 2002 does the concentration of iron fall below the detection limit in the upper $10 \mathrm{~cm}$. This rapid drop in dissolved iron is likely due to the presence of a two long annelid worms found at this depth. These worms likely irrigate their burrows and maintain a more oxygenated environment in the vicinity. The lack of Fe at this depth is accompanied by a reduction in phosphate, ammonium and dissolved silica but a peak in nitrate. This suggests that nitrification was occurring within the burrow environment. 
Every core that was sectioned at this site contained a large number of worms, down to the extent of coring. Sectioning the core in a glove bag leads to a restricted view of the sediments and so did not allow accurate quantification of the number of organisms, but in all cores at this site at least 3 worms would be encountered. This is consistent with the observations of Wheatcroft et al. [1994] who found an abundant community of benthic organisms in the fine grained sediments of Massachusetts Bay. The activity of the benthic organisms may be responsible for the minima in porewater iron observed in most cores. Wheatcroft et al. [1994] using labeled particles found particles were redistributed to specific depths that were consistent between three cores and varied depending on the season. Reverse conveyor belt feeding and head-down deposit feeding were identified as the likely feeding methods leading to the observed tracer profiles. These mechanisms could bring oxygenated water down to depth, so oxidizing any dissolved $\mathrm{Fe}^{2+}$ present. In addition, surfical, oxic sediments would be transported downwards, leading to reduction of iron oxides and a localized release of iron to the porewaters. The net effect of bioturbation is thus difficult to determine and likely contributes to the variability observed between replicate cores.

There is no distinct seasonal oscillation in the depth of iron first released to the porewaters and the gradient in iron in the surface porewaters is relatively constant in all Massachusetts Bay cores. There is a late February - early April phytoplankton bloom observed at this site, as well as a strong bloom in fall [Keller et al., 2001]. Although the prevailing currents redistribute some of the material away from the site, it would be expected that there would still be a seasonal change in the delivery of organic matter to this site. The absence of a seasonal signal in the porewater iron may be due to the abundance of benthic organisms, with bioturbation and irrigation activities obscuring the signal.

\section{Solid Phase}

The solid phase data for Fe and Mn at the Massachusetts Bay site are shown in Figure 3-10, along with porewater data. A comparison of the solid phase data for both sites is given in Figure 3-11. Surface enrichments in Fe and $\mathrm{Mn}$ are more distinct at this site, with a $35 \%$ and $14 \%$ enrichment of $\mathrm{Fe}$ and $\mathrm{Mn}$ respectively, extending deeper than in Hingham Bay, to a depth of $2 \mathrm{~cm}$ for iron. The depth of the surface enriched layer lies above and extends into the zone in which $\mathrm{Fe}$ is released to the porewaters. This is consistent with the porewater flux supplying iron to the surface enriched layer. The enriched layer extends into the zone 


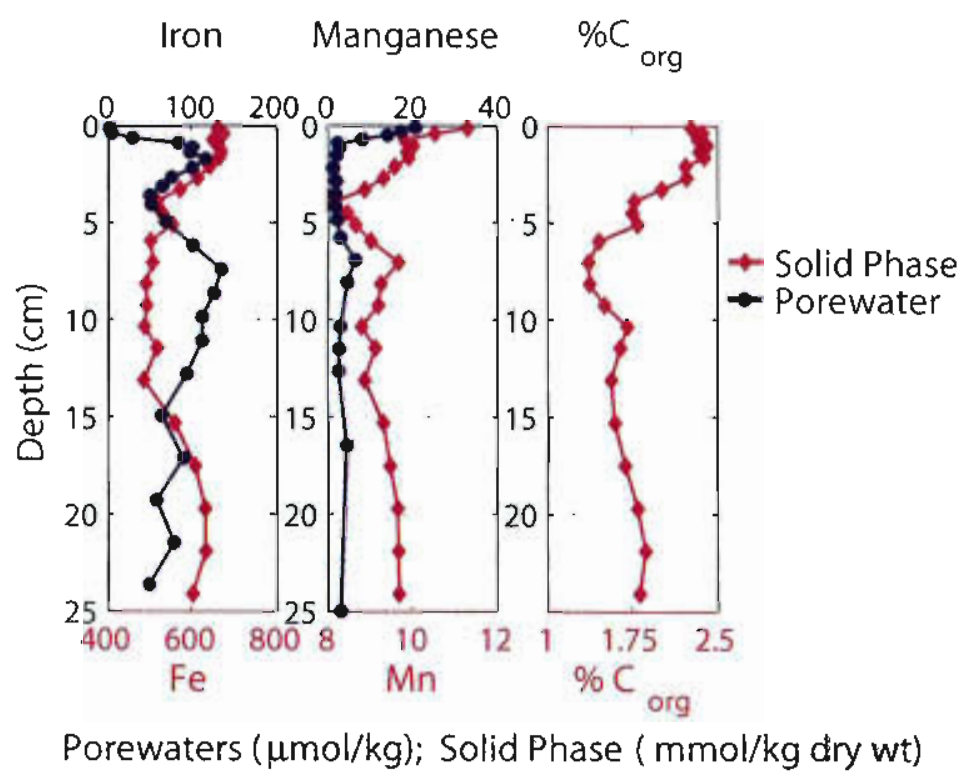

Figure 3-10: Solid phase iron, manganese and \% organic carbon profiles for Massachusetts Bay February 2002 (Core 3). The porewater profles of iron from Core 1 February 2002 and manganese from September 2003 are also shown.

of dissolved Fe release and this may suggest the solid and porewater phases are in slight disequilibrium. However the solid phase enrichment persists down to the depth of ${ }^{234} \mathrm{Th}$ penetration into the sediments (pers. comm. W. Martin) indicating that the sediments at this depth are mixed over a timescale of 100 days. If reductive dissolution of iron oxides is slower than the timescale of mixing, the solid phase and porewater profiles will appear to be in disequilibrium. A modeling approach to assess if realistic reaction rates would allow this disequilibrium to develop is required to assess if this is a plausible explanation. An alternative explanation may be that the porewaters are reflecting a recent change in the organic matter flux. If the sediments had recently become more reducing the solid phase profile would lag behind the more sensitive and faster responding porewater phases. However, this is not the case at this site as oxygen profiles collected by Fred Sayles since 1995 show no change in the oxygen penetration depth and benthic chamber data collected since 2001 show no change in the oxygen consumption rate (pers.comm. W.R. Martin).

Having described the early diagenetic reactions of iron and manganese at each site and investigated the seasonal variation in redox chemistry, the goals of the following sections are to characterize the cycling of silver, lead and copper at each site under the range of conditions encountered in each season. 
Table 3.3: NOAA Sediment Quality Criteria [Buchman, 1999]

\begin{tabular}{lcc}
\hline$\left(\mu \mathrm{mol} / \mathrm{kg}_{\alpha r y w t}\right)$ & Threshold Effects Level & Probable Effects Level \\
\hline Copper & 290 & 1700 \\
Lead & 150 & 540 \\
Silver & 7 & 16 \\
\hline
\end{tabular}

\subsubsection{Trace Metal Solid Phase Distributions}

The total digestion solid phase data for both Hingham Bay and Massachusetts Bay are shown in Figure 3-11. Additional solid phase data for both sites are shown in Figures 3-39 to $3-43$, provided at the end of the Chapter. The dominant feature of all silver, copper and lead profiles for Hingham Bay is the increase in concentration with depth. This reflects the historical pollutant loadings at depth, recent reductions in metal discharges and dilution by cleaner material towards the surface, and loss from the sediments. The profiles are consistent with the observations of Bothner et al. [1998] that the sediment surface concentrations at this site have decreased over the period 1977-1993. Despite the evident decreases the concentration of these metals is still high, with all metals exceeding the NOAA threshold effects level [Buchman, 1999], which represents the level below which adverse effects would be expected only rarely. The silver concentration in the surface sediment is $20 \mu \mathrm{mol} / \mathrm{kg}$ and exceeds the probable effects level of $16 \mu \mathrm{mol} / \mathrm{kg}$ [Buchman, 1999], the level at which adverse effects are frequently expected. The NOAA sediment quality criteria for silver, lead and copper are given in Table 3.3. The surface silver level exceeds the highest silver concentration reported from contaminated sites in San Francisco Bay by Rivera-Duarte and Flegal [1997a].

The Massachusetts Bay solid phase profiles are quite different from those at Hingham Bay. Like the iron and manganese profiles, the lead, silver and copper data all show distinct surface enrichments in the upper $2 \mathrm{~cm}$, with silver enriched by $37 \%$, copper by $35 \%$ and lead by $25 \%$ relative to deeper sediments. Below the enrichment peak the concentrations are relatively constant with depth. Due to the surface enrichments of lead at the MB site the concentrations in the surface sediments at Hingham Bay and Massachusetts Bay are similar, while for silver and copper the surface sediments at Massachusetts Bay are 30\% and 60\% of the concentrations in Hingham Bay. The trace metal enrichments in MB are co-incident with those of iron, manganese and organic carbon. Sediment trap data for Massachusetts 


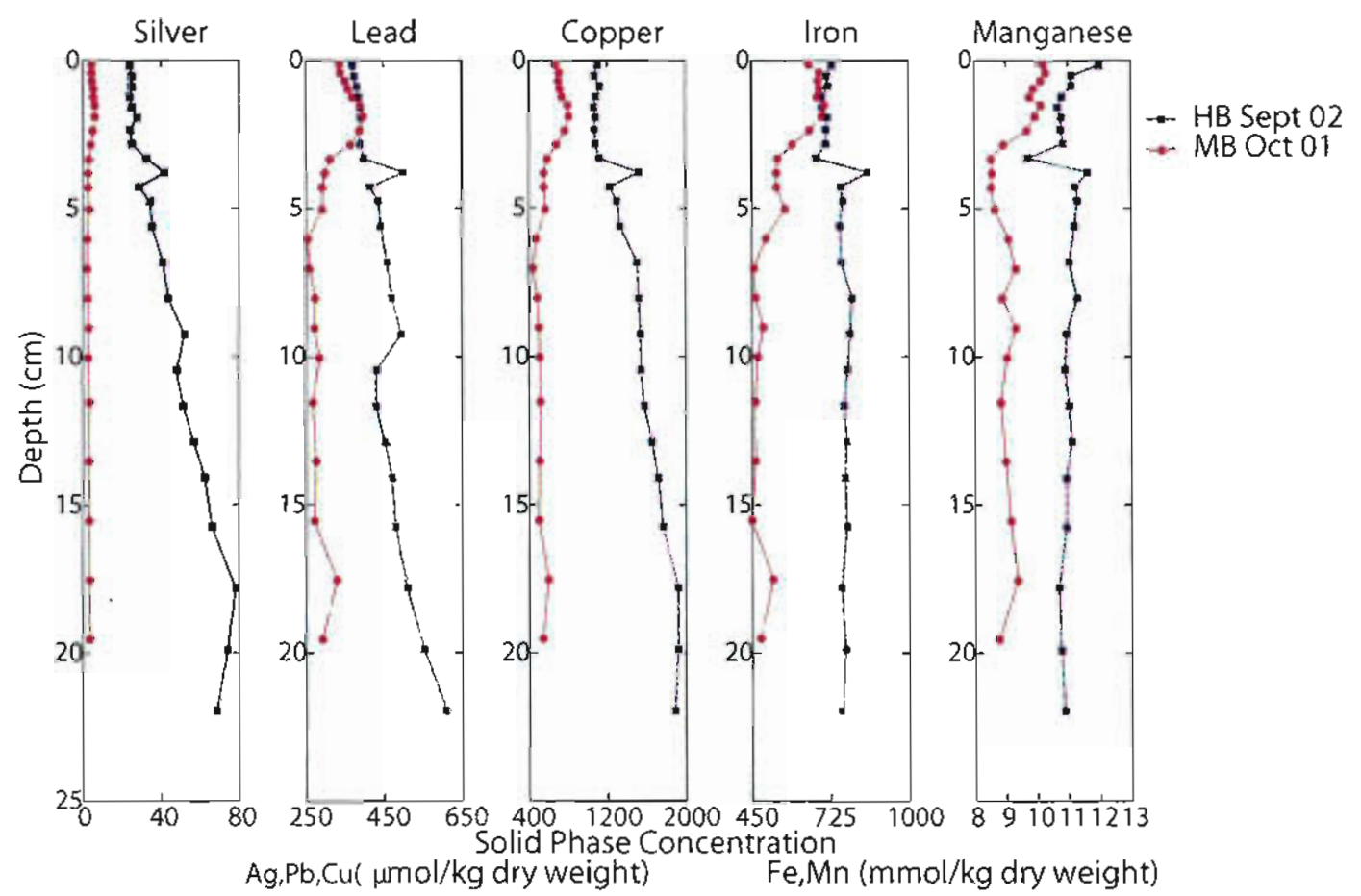

Figure 3-11: Solid phase profiles of for silver, lead, copper, iron and manganese for Massachusetts Bay October 2001 and Hingham Bay September 2002).

Bay, collected by Dr Michael Bothner of the USGS shows that the surface sedirnents are enriched in iron, copper, silver and lead relative to particles collected in the sediment trap. This contrasts with the behavior of organic carbon, which is depleted in surface sediments relative to the sediment trap data. The trace metals must thus cycle differently than organic carbon. The coincident enrichments of the trace metals with those of iron and manganese at the MB site strongly suggests that the cycling of these metals is associated with the mechanisms of iron and/or manganese cycling. The porewater profiles from each site will now be discussed to further investigate some of the possible mechanisms.

\subsubsection{Trace Metal Porewater Distributions}

The following section aims to characterize the porewater profiles of lead, silver and copper and to determine the changes in metal cycling at each site over the course of a year. All the data from Hingham Bay will be presented and then the silver profiles will be discussed in detail. The copper and lead data will then be compared to the silver data. The data from the Massachusetts Bay site will then be presented and the two sites compared. 


\subsubsection{Hingham Bay}

The porewater profiles of silver, lead and copper for Hingham Bay for winter (Figures 3-12 and 3-13), late spring and summer (Figures 3-14, 3-15, 3-18 and 3-19) and fall (Figures 316 and 3-17) are given below. The concentrations of silver measured in the porewaters ranged from $0.04-1.2 \mathrm{nmol} / \mathrm{kg}$ (detection limit for analyses $0.006 \mathrm{nmol} / \mathrm{kg}$, see Table 2.4). These concentrations fall below the detection limit of $2 \mathrm{nmol} / \mathrm{kg}$ obtained for the first silver determinations in porewater by Lyons and Fitzgerald [1983]. Most of the samples in the study by Lyons and Fitzgerald [1983] reported the silver concentrations at two sites in Long Island Sound to be below their detection limit, however, a maximum concentration of $9 \mathrm{nmol} / \mathrm{kg}$ was reported. The only other study measuring silver in porewaters is that of Rivera-Duarte and Flegal [1997a], in which 9 sites in San Francisco Bay and environs were investigated. The concentrations reported ranged from $0.004-4.6 \mathrm{nmol} / \mathrm{kg}$ with the average concentration of $0.1 \pm 0.1 \mathrm{nmol} / \mathrm{kg}$. The concentrations in the solid phase at Hingham Bay are higher than those found in San Francisco Bay, so although the porewater concentrations measured here do fall within the range reported in San Francisco Bay, it is also useful to compare the partition coefficient $D$, of silver between the porewater and the solid phase, calculated by the following equation:

$$
D_{M}=\frac{C_{M}^{s e d}}{C_{M}^{p w}}
$$

where the partition coefficient for a particular metal $M$ is defined by the concentration of metal in the sediment $C_{M}^{\text {sed }}\left(\mathrm{mol} / \mathrm{kg}\right.$ dry weight) and the concentration in the porewater $C_{M}^{\text {pv }}$ (mol/L). The partition coefficient determined by Rivera-Duarte and Flegal [1997a] used an aqua-regia digestion to determine sediment concentration, so may have not quantitated all the silver phases determined in this study, in which HF was used in addition to aqua regia. However their recoveries were good, so the silver concentrations in the silicate phase that the aqua regia digestion did not extract was small. Rivera-Duarte and Flegal [1997a] report a range of $\log _{10} D_{A g}$ values for the silty and clayey sediments of San Francisco Bay of 2.1 to 5.8 , with an average of 4.9 . These compare well with Hingham Bay values which range from 4.3 to 5.6 .

The copper concentrations found in this study range from 4 to $45 \mathrm{nmol} / \mathrm{kg}$. These values fall within the range reported by other authors for a range of sediment redox conditions in 
Narragansett Bay (10-400 nM) [Elderfield et al., 1981], Southern Californian Borderlands (0-120 nM) [Shaw et al., 1990], the Tyrrhenian Sea (8-47 nM) [Ciceri et al., 1992] and San Francisco Bay (0.5-160) [Rivera-Duarte and Flegal, 1997b]. The partition coefficient $\log _{10} D_{C u}$ for Hingham Bay ranges from 4.3 to 5.6. The porewater concentrations of lead ranged from 0.3-24 nmol/kg. Those determined by Rivera-Duarte and Flegal [1994] in San Francisco Bay ranged from 0.07 to $19 \mathrm{nmol} / \mathrm{kg}$, the concentrations found in the Tyrrhenian Sea ranged from 0.5 to $12 \mathrm{nmol} / \mathrm{kg}$ [Ciceri et al., 1992] and a single site in the Chesapeake Bay the concentration range was 10 to $28 \mathrm{nmol} / \mathrm{kg}$ [Shea and Maccrehan, 1988]. The partition coefficient $\log _{10} D_{P b}$ for lead in San Francisco Bay ranged from 3.7 to 6.6, with an average of 4.9 , and the values for Hingham Bay range from 4.7 to 5.7 , so as for silver, they fall within the range previously observed.

\section{Silver Porewater Profiles}

In each season the silver porewater profiles have a number of peaks, representing release and removal processes. These peaks occur in distinct zones throughout the year, and the following discussion will focus on the changes in each zone over the seasons. The four zones which will be discussed are 1) the sediment-water interface and underlying zone with no dissolved iron in the porewaters (referred to in the discussion below as the 'oxic zone', where oxic in this context indicates that iron oxides are not being reduced; oxygen and/or nitrate are present); 2) the sub-oxic zone in which there is dissolved iron in the porewaters 3 ) the sulfide interface and 4) the anoxic zone with sulfide in the porewaters. The first two regions show a distinct seasonal variation. Due to their proximity to the sediment-water interface they are the most important for driving remobilization of metals from the sediments and so will be discussed first. 

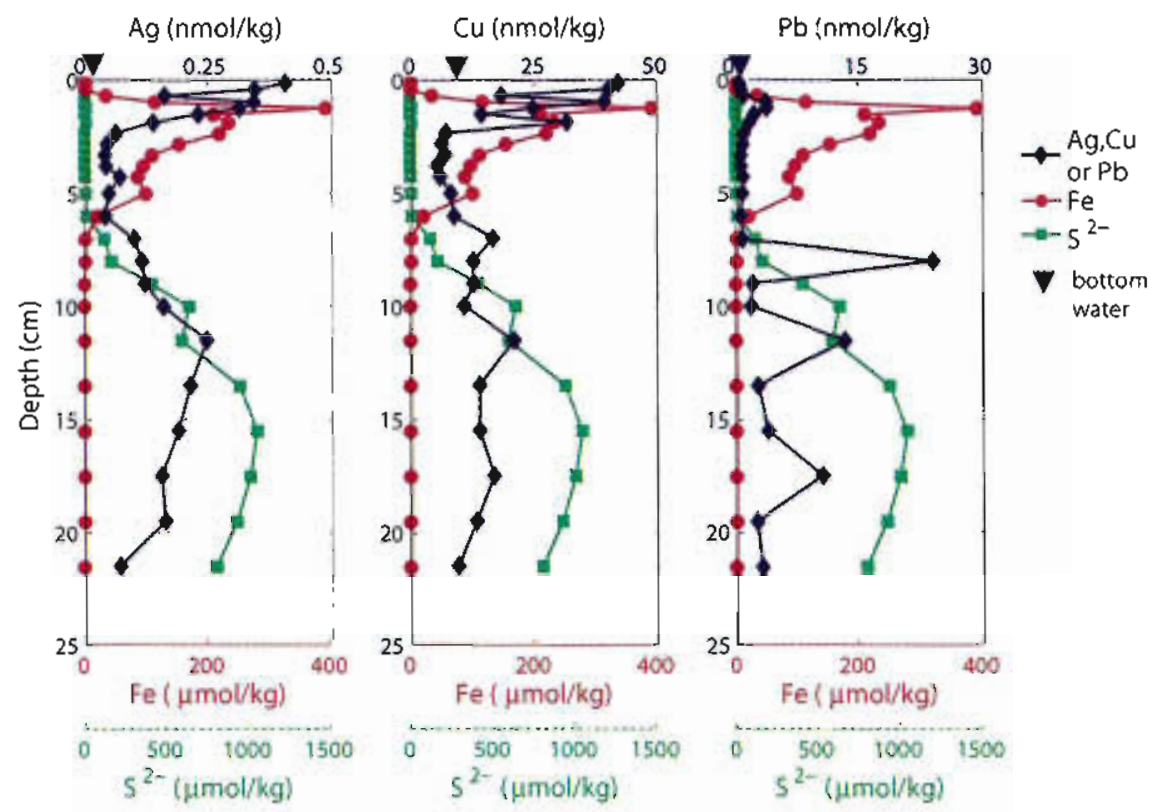

Figure 3-12: Porewater profile of silver, lead and copper for Hingham Bay January 2002 Core 1. Iron and sulfide data are also shown on each panel.
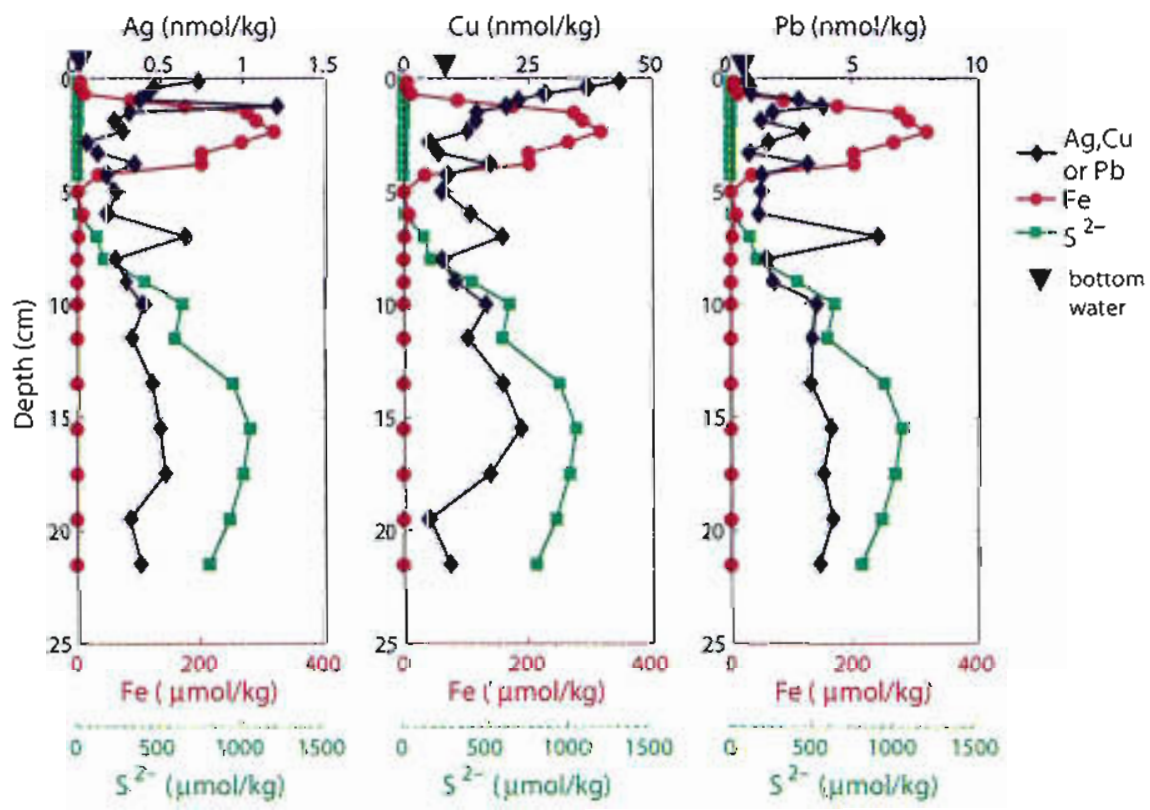

Figure 3-13: Porewater profile of silver, lead and copper for Hingham Bay January 2002 Core 2. Iron and sulfide data are also shown on each panel. 

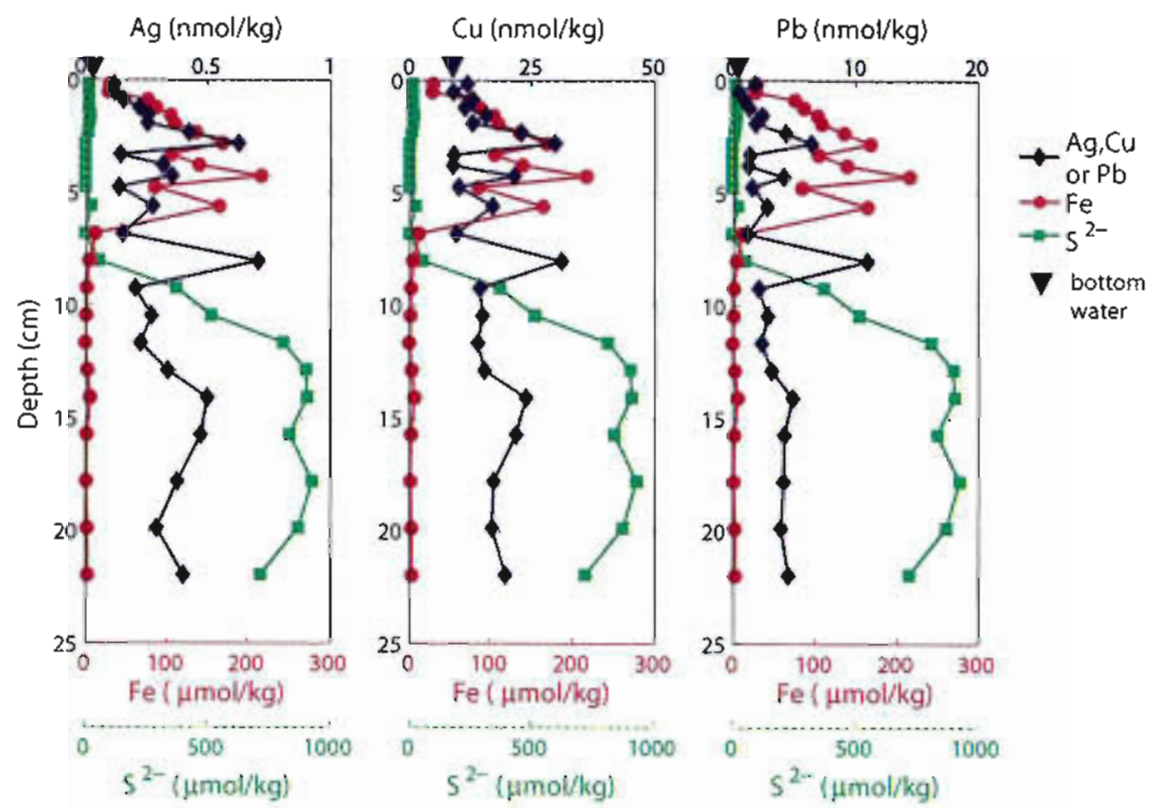

Figure 3-14: Porewater profile of silver, lead and copper for Hingham Bay July 2002 Core 1. Iron and sulfide data are also shown on each panel.
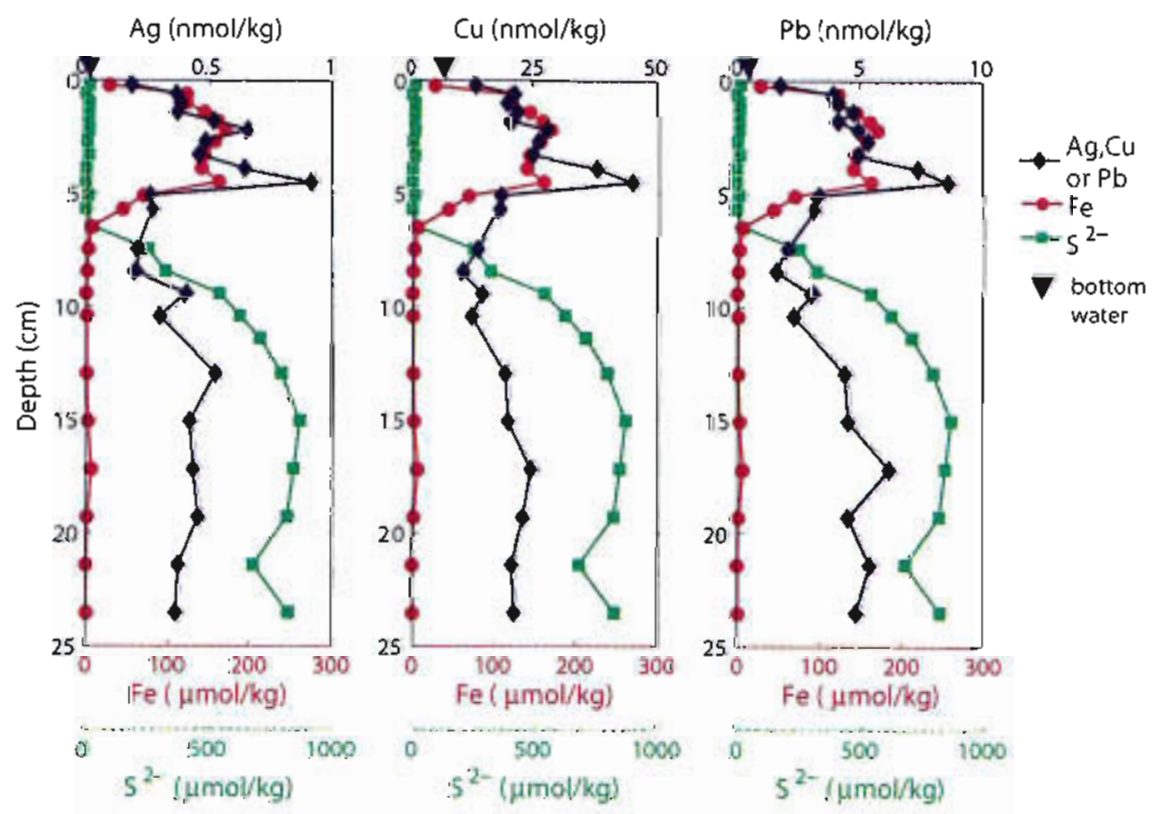

Figure 3-15: Porewater profile of silver, lead and copper for Hingham Bay July 2002 Core 2. Iron and sulfide data are also shown on each panel. 

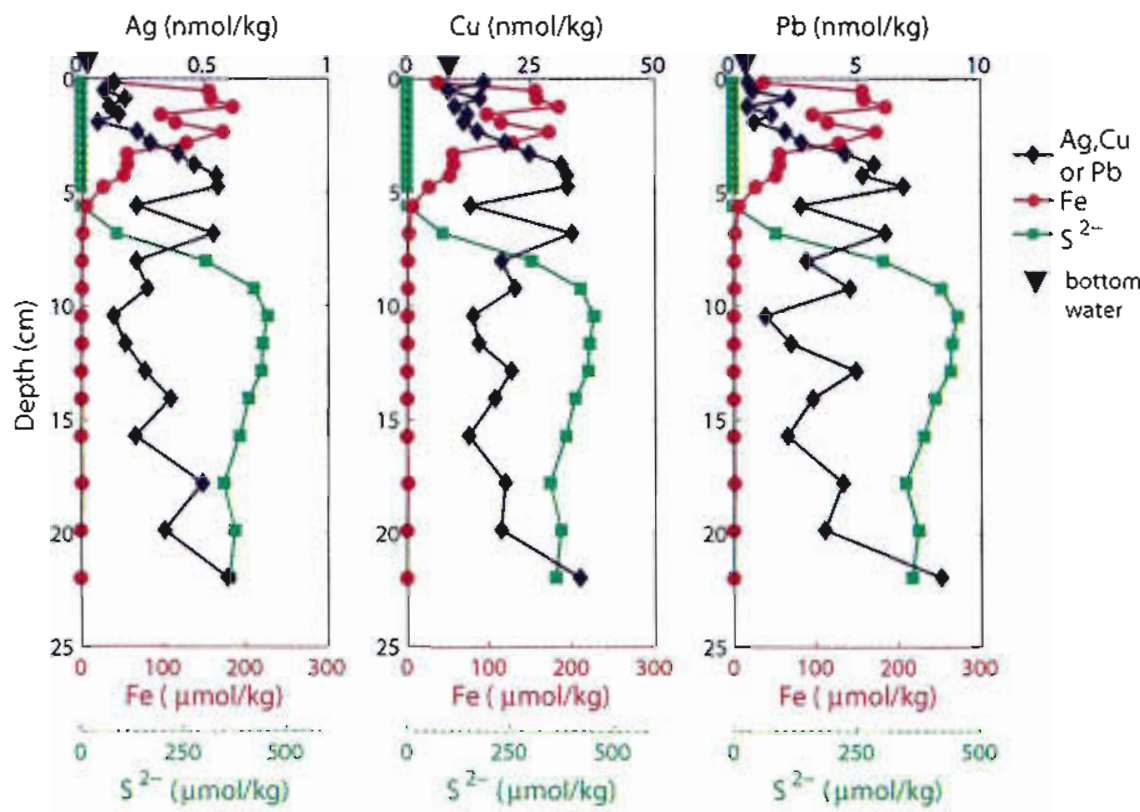

Figure 3-16: Porewater profile of silver, lead and copper for Hingham Bay September 2002 Core 1. Iron and sulfide data are also shown on each panel.
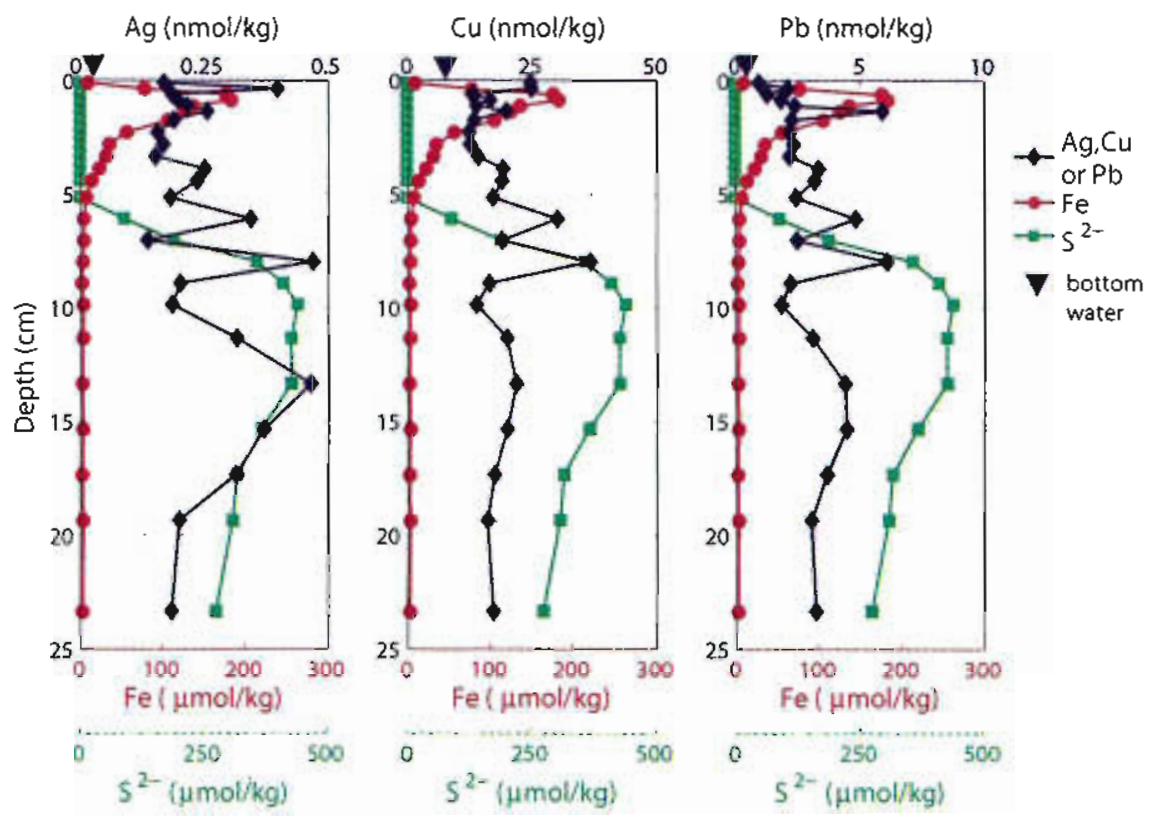

Figure 3-17: Porewater profile of silver, lead and copper for Hingham Bay October 2003 Core 1. Iron and sulfide data are also shown on each panel. 

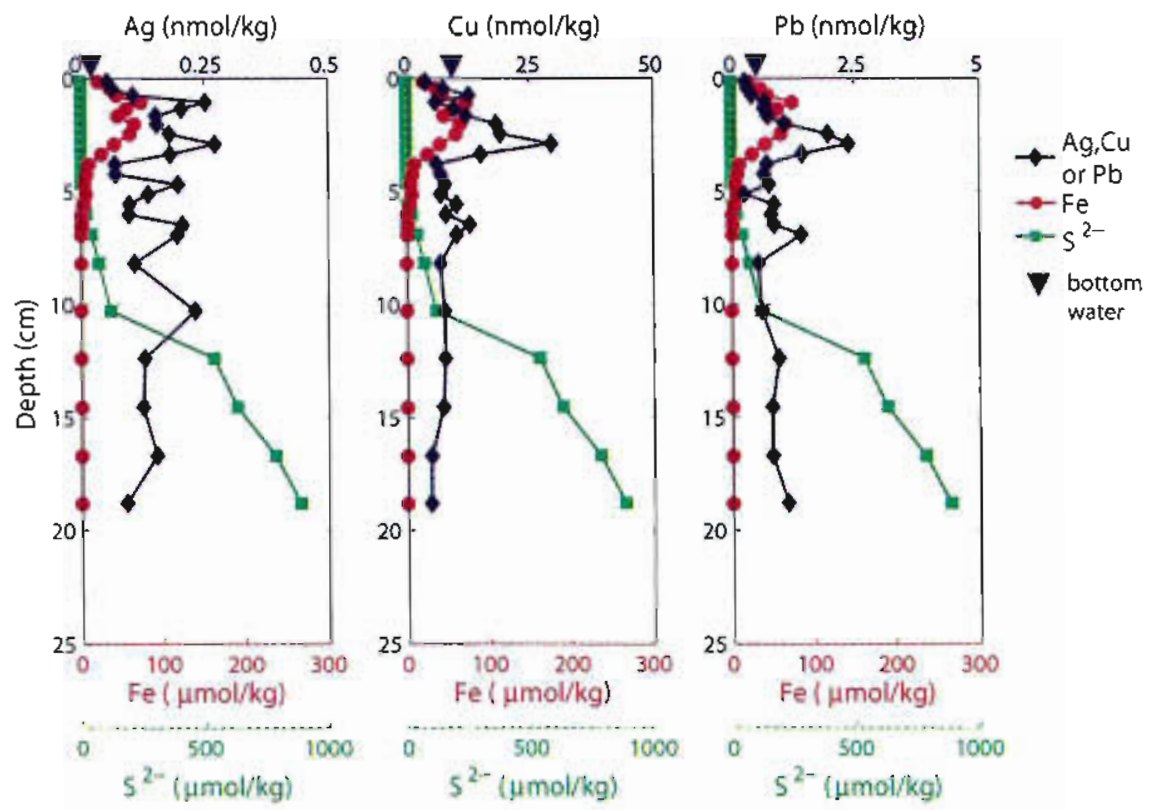

Figure 3-18: Porewater profile of silver, lead and copper for Hingham Bay June 2004 Core 1. Iron and sulfide data are also shown on each panel.
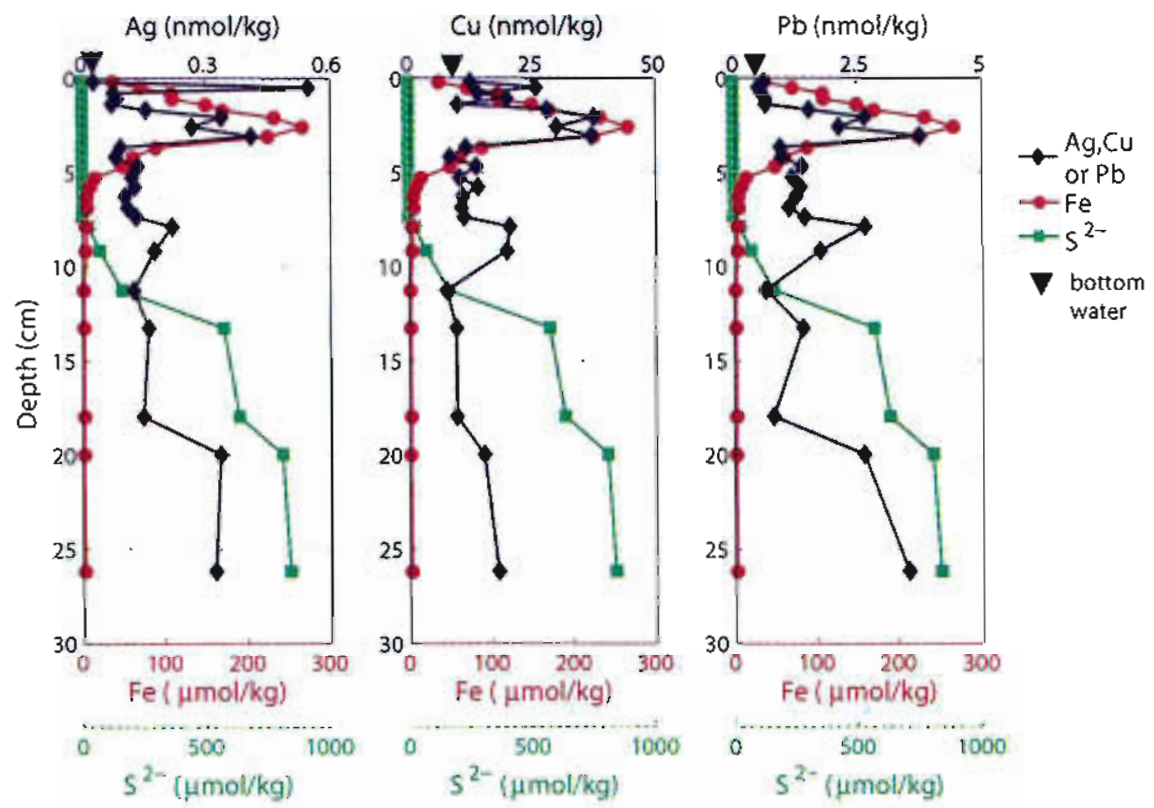

Figure 3-19: Porewater profile of silver, lead and copper for Hingham Bay June 2004 Core 2. Iron and sulfide data are also shown on each panel. 
In winter both sediment cores (Figure 3-13 and 3-12) show a distinct release of silver at the sediment-water interface. At all other times of the year the silver concentration is above the bottom water silver concentration (measured at $0.02 \pm 0.001 \mathrm{nmol} / \mathrm{kg}$ for June 2004) but such a strong release exceeding $0.2 \mathrm{nmol} / \mathrm{kg}$ is only observed in January. Releases of metals such as copper, cadmium and vanadium at the interface have been attributed to the degradation of either biogenic material or particles which scavenged metals from the water column [Klinkhammer, 1980; Shaw et al., 1990]. The release of silver observed here may also be due to the degradation of freshly deposited particles; however the nature of the silver association with these particles is unknown. In the past the silver deposition was associated with sewage wastes [Bothner et al., 1998]; however with the cessation of sewage sludge release to Boston Harbor, the source of silver is less evident. Silver has oceanic profiles closely related to silica [Martin et al., 1983; Flegal et al., 1995] and is considered to associate with diatoms [Flegal et al., 1995; Boning et al., 2004] in the oceans. There are number of winter and spring diatom species in Massachusetts Bay [Hegarty and Villareal, 1998] that may be responsible for delivery of silver to the sediments, however, diatoms are unlikely to be the most abundant settling material at this coastal site due the the proximity to terrestrial sources of sediments. Silver scavenged onto other particles within the water column is likely to be more a more important source of silver to the sediments, a mechanism also suggested to occur in the ocean [McKay and Pedersen, 2000]. The source of this silver may be from the sewage effluent released in Massachusetts Bay, or, as the concentration in the porewaters exceeds the concentration in the water column, the silver may be recycled and derived from benthic sources (see Section 3.3.8).

That the strongest release of silver at the sediment-water interface is observed in winter may also be due to the oxidation of reduced silver species. From the January iron parewater profiles it is evident that the sediments at this time are in their least reducing state, as, unlike at any other time of the year, there is no dissolved iron measured in the upper $0.5 \mathrm{~cm}$ porewaters. Thus any silver sulfides or other reduced species that may have been mixed upwards or formed over the summer may be oxidized in the winter, releasing silver to the porewaters. The silver released at the sediment-water interface will flux both upwards into the overlying water and downwards into the sediments. Within the sediments it is removed rapidly above the sub-oxic zone. This is most likely due to adsorption by the iron oxyhydroxides that are present in the oxic zone. Further evidence for the silver association 
with iron oxyhydroxides is obtained from the porewater profiles within sub-oxic zone, and this is discussed below.

In all the late spring and early summer profiles, July 2002 (Figures 3-14 and 3-15) and June 2004 (Figures 3-18 and 3-19) there is a distinct release of silver in the iron reduction zone. The silver profiles closely track the iron profile while there is iron measurable in the porewaters. The silver profiles follow the iron profiles during both release and removal of iron, including the sharp depression generated due to irrigation activity of benthic organisms. There is a linear relationship between the iron and silver concentration within the zone of iron release, for example the correlation coefficient between the two species in the July 2002 Core 1 is $r=0.90$. When all the data from this zone for June and July is pooled together $r=0.6$, which for 40 points is a significant linear relationship. The similarity in the profiles suggests that silver was associated with solid phase iron oxides and released to the porewaters during reductive dissolution of the iron. Iron and manganese oxides are well known to adsorb a large range of trace metals [Jenne, 1968; Balistrieri et al., 1982]. Similarities in the porewater profiles between iron, nickel and vanadium have similarly been used to infer scavenging by iron oxyhydroxides [Shaw et al., 1990]. Rivera-Duarte and Flegal [1997a] noted that the removal of porewater silver in San Francisco Bay was coincident with the removal of manganese from the porewaters and so suggested that the silver was associating with manganese phases in the sediments. However the sampling resolution employed was low and neither the manganese nor iron porewater data were actually presented, so it is not possible to assess if there was also evidence of an association with an iron oxide phase or if the redox zones at some sites are too compressed to identify a difference. The manganese porewater profile for Hingham Bay in July shows a release in the upper $2 \mathrm{~cm}$ of sediment (Figure 3-2) and bears no relationship to the silver profile, suggesting that silver is not coupled to the manganese cycle at this site.

Direct evidence of silver adsorption to iron oxides is available for synthetic model systems. Silver(I) was shown to adsorb to amorphous iron hydroxides (ferrihydrite) at values of $\mathrm{pH}>6$ [Davis and Leckie, 1978]. However the addition of chloride ions decreased the adsorption due to formation of $\mathrm{AgCl}_{2}^{-}$complexes in solution. Addition of thiosulfate [ $\mathrm{Ong}$ and Leckie, 1998] and model organic ligands selected to mimic humic substances [Davis and Leckie, 1978] led to an enhancement of adsorption. Silver-chloro complexes are expected to be the dominant inorganic species in oxic seawaters; however, the high levels of dissolved 
organic compounds [Burdige and Gardner, 1998; Skrabal et al., 1997] in porewaters as well as trace levels of sulfide [Rosenthal et al., 1995] will be important in the speciation and may thus enhance the adsorption of silver onto iron oxides. Wen et al. [1997] and Herrin et al. [2001] found an association between silver and iron in estuarine and riverine particulates respectively. Wen et al. [1997] conducted a radiotracer experiment with silver and hematite in seawater and concluded that the association of silver with iron oxides was minimal, and attributed the relationship observed in the particles to silver associating with iron sulfide phases. However hematite is a crystalline phase and will have a reduced affinity for trace metals relative to the amorphous phase, and it is the amorphous phase that is the most reactive fraction of the iron oxide pool in coastal sediments [Canfield, 1989]. The concentrar tions of silver used in both the experiments of Davis and Leckie [1978] and Wen et al. [1997] was over $100 \mathrm{nM}$. As the dissolved concentrations in the porewater are two orders of magnitude lower than the experimental concentrations these results do not discount sorption of low levels of silver to iron oxyhydroxides. Further experiments to confirm the porewater observations that adsorption of $\mathrm{nM}$ levels of silver to iron oxyhydroxides in a seawater does occur are required.

Although the late spring and early summer profiles consistently indicate a tight coupling between iron and silver the profiles from fall (Figures 3-16 and 3-17) do not exhibit such behavior. In the September profile the silver data show no relationship to the iron concentrations $(r=0.29)$.

In January (Figures 3-12 and 3-13) the silver response within the sub-oxic zone is intermediate between the fall and late spring/early summer profiles. There is a very strong release of silver at $1.2 \mathrm{~cm}$ depth in Core 2 , coincident with the first release of iron to the porewaters. The highest silver concentration, $1.2 \mathrm{nmol} / \mathrm{kg}$, measured in this study is associated with this peak. This peak is consistent with the observations in July (Figures 3-14 and 3-15) that silver associated with iron oxyhydroxides is released to the dissolved phase upon reductive dissolution of the oxide. Unlike the July profile however the silver profile does not continue to track the iron profile with depth. Despite continued release of Fe to the porewaters down to a depth of $4 \mathrm{~cm}$ the silver reaches relatively steady and low levels by $2.5 \mathrm{~cm}$, behavior similar to that observed in the fall. The observations from the oxic and suboxic zones in each season will be complied into a conceptual model presented in the following section. Before this is introduced however the silver behavior in the remaining 
two zones, the sulfide interface and the anoxic zone will be described.

The sulfide interface, the point at which the iron and sulfide concentrations are both zero, is the depth below which the sulfide concentration builds up rapidly. In all but one of the profiles collected (January 2002 Core 1, Figure 3-12) there is a consistently strong release of silver close to the sulfide interface (this peak is at $7 \mathrm{~cm}$ in January 2002 Core 2 (Figure 3-13), $8 \mathrm{~cm}$ in July 2002 Core 1 (Figure 3-15) and $7 \mathrm{~cm}$ in September Core 1 (Figure 3-16)). In some profiles, such as the October 2003 profile (Figure 3-17), there are multiple peaks around the interface. Speciation calculations, described in more detail in Chapter 4 indicate that the silver concentration within the interface peaks is up to 100 times over-saturated with respect to silver sulfide, if inorganic speciation alone is included. Investigations of the concentrations of strong silver-binding ligands found in the sediments at Hingham Bay, discussed in Chapter 4, have shown that thiols and polysulfides are not present at high enough concentrations to be responsible for the enhanced solubilization at the interface peak.

Some other unidentified strong ligand, generated close to the sulfide interface, may be responsible for the strong release of silver. However the sulfide interface is a highly dynamic region where the iron flux from above is balanced by the sulfide flux from below. In this region there will be intense formation of iron-sulfide species and changes in the redox conditions driven by changes at the sediment-water interface will lead to readjustments of the depth of the interface. The interface is thus an ideal location at which to expect the formation of colloidal sized metal sulfides. Metal-sulfide colloids have been identified in laboratory work to form when solutions become oversaturated with respect to the solid phase metal-sulfide [Sukola et al., 2005]. The colloids were found to be dynamic and eventually coagulated to form a precipitate. In porewaters it may be expected that organic material may lead to an additional stabilization of the colloids, so that they could be a persistent feature at the sulfide interface. Multinuclear metal sulfide clusters, as identified in rivers by [Rozan et al., 2000a], which are stable dissolved structures that appear to be resistant to destruction may also account for the supersaturations of silver. The existence of metal clusters in the environment has however recently been questioned [Sukola et al., 2005] and may be a freeze drying artifact.

These data cannot assess whether the sulfide interface peak is due to colloidal sulfides or dissolved metal clusters. Filtering a porewater samples with a $0.02 \mu \mathrm{m}$ filter was considered, 
as such samples would have provided insight into which of these explanations was more plausible. However, the membranes could not be cleaned to a sufficient level to enable uncontaminated filtration of small volumes of porewater. The stability of both colloids and clusters, if one of these is the cause of the enhanced silver solubilization, may also account for the multiple peaks observed in some profiles. If the sulfide interface had migrated recently and then stabilized at a new level, the additional peaks may be relics, persisting at the previous interface depth.

Below the sulfide interface, within the anoxic zone, for most cores the silver profile becomes smooth and relatively stable with depth. The only exception to this is Core 1 of September 2002, which shows an increase in silver with depth in a stepwise fashion. This is also the only core that shows an oscillation in $\mathrm{pH}$ within the anoxic zone (see Figure 3-31), and this may be affecting the silver values. The average silver concentrations below $15 \mathrm{~cm}$ for each core are shown in Table 3.4. Also shown in Table 3.4 are the concentrations of total dissolved silver that are calculated to be in the porewaters if they are assumed to be in equilibrium with an $\mathrm{Ag}_{2} \mathrm{~S}$ solid phase. Inorganic sulfide complexes are expected to dominate the speciation [Lyons and Fitzgerald, 1983]. The measured values of silver range from $10 \%$ to $80 \%$ of the calculated values. This thermodynamic calculation has a bigh degree of uncertainty due to the uncertainty in the thermodynamic data and the possible differences between the actual sediment phase controlling silver solubility and the thermodynamic data, which is for a pure crystalline phase solid. Due to the large uncertainties it is not possible for this Hingham Bay data to distinguish between the silver being saturated or slightly undersaturated with respect to silver sulfide.

\section{The Seasonal Benthic Silver Cycle}

The very different relationships between iron and silver in each season in both the oxic and sub-oxic zones are likely due to the seasonal transfer of silver between different sedimentary phases. The observations from each season are compiled into a conceptual model of the seasonal silver cycle which is now discussed. A schematic diagram of the seasonal silver cycle inferred from the porewaters is given in Figure 3-21. The iron cycle that forms the backbone of the silver cycle is given in Figure 3-20 for reference. In these figures the redox zones discussed in the previous section are depicted as horizontal lines. The three phases considered important in the cycling of metals are a sulfide phase, an oxide phase and a 
Table 3.4: Average Trace Metal and Sulfide Concentrations Below $15 \mathrm{~cm}$ in Hingham Bay

\begin{tabular}{|c|c|c|c|c|c|c|c|c|}
\hline \multirow[b]{2}{*}{ Core } & \multicolumn{3}{|c|}{ Measured } & \multicolumn{2}{|c|}{ Measured } & \multicolumn{3}{|c|}{ Calculated $^{1}$} \\
\hline & $\mathrm{Ag}$ & $\begin{array}{c}\mathrm{Pb} \\
\mathrm{mol} /\end{array}$ & $\mathrm{Cu}^{2}$ & $\underset{(\mu \mathrm{M})}{\mathrm{S}}$ & $\mathrm{pH}$ & $\mathrm{Ag}$ & $\begin{array}{c}\mathrm{Pb} \\
\mathrm{mol} /\end{array}$ & $\underset{\mathrm{gg})}{\mathrm{Cu}}$ \\
\hline $\operatorname{Jan} 02$ Core 1 & 0.14 & 5.1 & 13.4 & 947 & 7.26 & $\overline{2}$ & 1.2 & 0.14 \\
\hline $\operatorname{Jan} 02$ Core 2 & 0.39 & 4.6 & 13.2 & 947 & 8.09 & 1 & 0.2 & 0.04 \\
\hline July 02 Core 1 & 0.39 & 4.3 & 19.1 & 839 & & & & \\
\hline July 02 Core 2 & 0.42 & 5.1 & 22 & 808 & & & & \\
\hline Sept 02 Core 1 & 0.41 & 4.7 & 21.7 & 368 & 7.40 & 0.5 & 0.4 & 0.02 \\
\hline Oct 03 Core 1 & 0.27 & 3.6 & 17.9 & 317 & 7.69 & 0.4 & 0.1 & 0.01 \\
\hline June 04 Core 1 & 0.12 & 0.92 & 5.6 & 770 & & & & \\
\hline June 04 Core 2 & 0.27 & 2.33 & 14.3 & 762 & 7.22 & 2 & 1.0 & 0.09 \\
\hline
\end{tabular}

${ }^{3}$ calculated total dissolved concentration in equilibrium with $\mathrm{Ag}_{2} \mathrm{~S}$, $\mathrm{CuS}$ and $\mathrm{PbS}$ at the measured $\mathrm{pH}$ and sulfide concentration.

${ }^{2}$ the ratio between $\mathrm{Cu}(\mathrm{I})$ and $\mathrm{Cu}(\mathrm{II})$ was determined by assuming the $\mathrm{SO}_{4}^{2-} / \mathrm{S}^{2-}$ couple controls the pe.

Thermodynamic constants taken from Morel and Hering [1993] with ion activity coefficients calculated using the Davies equation. Data for $\mathrm{Pb}$ and $\mathrm{Cu}(\mathrm{I})$ aqueous sulfide complexes from Al-Farawati and van den Berg [1999] for $\mathrm{Pb}$ and Saito et al. $[2003]$ for $\mathrm{Cu}(\mathrm{I})$.

dissolved phase, and these are represented by the vertical lines. Each compartment within the figure thus represents a particular phase within a redox zone. The transfers between phases are shown by the arrows.

For the iron cycle there is an oscillation in the depth of the redox boundaries over the course of a year and so the inventory in each phase and the magnitude of the transfer between phases will differ on a seasonal basis. However there is always a release of iron to the porewaters and the dissolved iron diffuses both upwards and downwards throughout the year. The cycle depicted in Figure 3-20 must thus operate throughout the year in order to sustain the profiles observed. Due to silver adsorption by iron oxyhydroxides the silver cycle must be linked to the iron cycle and so Figure 3-21 is of the same form as Figure 320. Unlike the iron porewater profiles however, the silver profiles vary significantly in each season. Silver profiles from the same season but in different years show the same features, so that there must be a silver cycle that operates on an annual basis. The silver concentration in the sediments is 3 to 4 orders of magnitude lower than the iron concentrations and silver sulfides are more insoluble than iron sulfides. Due to these differences in chemistry and concentration silver becomes focussed within a compartment and cannot cycle with iron in all seasons. Instead, the silver cycle has dominant pathways in different seasons that over 


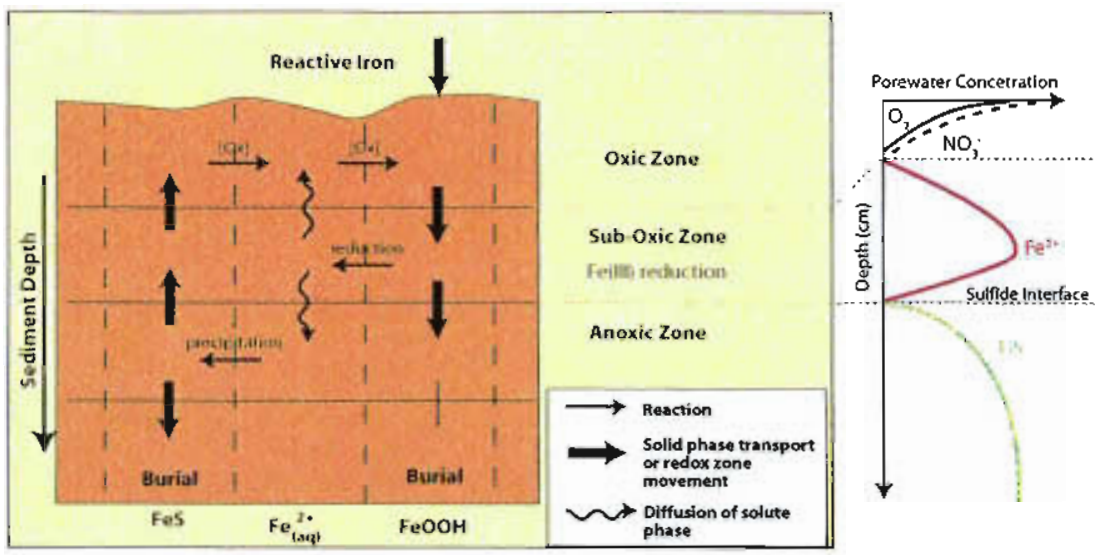

Figure 3-20: The iron cycle in Hingham Bay. Each box represents a particular sediment phase within a redox zone. The arrows represent the mecharism by which material can be transferred between boxes. Solid phase transport includes both bioturbation and sedimentation. A schematic porewater profile is also shown.

the course of the year have a similar form to the iron cycle.

In late spring as the greater demand for oxidants drives the redox horizons upwards, iron oxyhydroxides that were stable over winter are reduced, releasing any adsorbed silver into the porewaters. Once in the dissolved phase the silver may encounter a sulfide anion, generated by sulfate reduction. Due to the extreme insolubility of silver sulfide $\left(K_{s p}\right.$ $\left(\mathrm{Ag}_{2} \mathrm{~S}\right)=10^{-50.1}$ [Morel and Hering, 1993]), and assuming no kinetic limitation to silver precipitation, the silver will precipitate out as a silver sulfide phase. Adams and Kramer [1998] found that the precipitation of $\mathrm{Ag}_{2} \mathrm{~S}$ is rapid and that even in the presence of FeS the silver precipitates out as a discrete phase. Organic or sulfide complexation of silver will stabilize more silver in the dissolved phase and this is addressed with speciation calculations in Chapter 4 . The removal of silver as a sulfide is the same mechanism proposed by Rosenthal et al. [1995] for the removal of cadmium in sub-oxic sediments. These authors suggest that even though there is no detectable sulfide in the porewaters, from thermodynamic calculations CdS is sufficiently insoluble $\left(K_{s p}(\mathrm{CdS})=10^{-27}\right.$ |Morel and Hering, 1993]) that trace levels of sulfide $(>0.01 \mu \mathrm{M}$ ) can lead to a supersaturation of the porewaters with respect to CdS. As silver sulfide is even less soluble than cadmium sulfide this mechanism seems even more applicable to silver. Additional evidence for the removal of silver as a sulfide phase at Hingham Bay is the removal of iron from the porewaters at depth most likely due to trace amounts of sulfide present within the sub-oxic zone. Silver sulfide is considerably more insoluble than FeS $\left(K_{s p}\left(\mathrm{FeS}_{\text {amorphous }}\right)=10^{-16.9}, K_{s p}\left(\mathrm{FeS}_{\text {mackinawite }}\right)=10^{-17.5}[\right.$ Aller, 
(a)
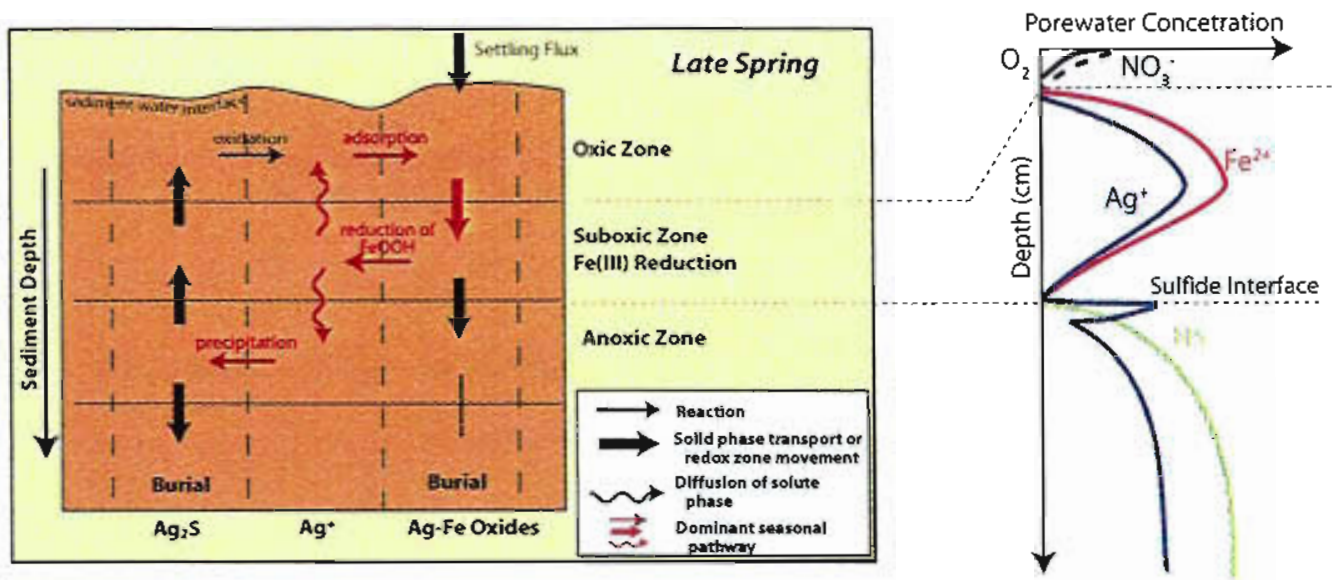

(b)
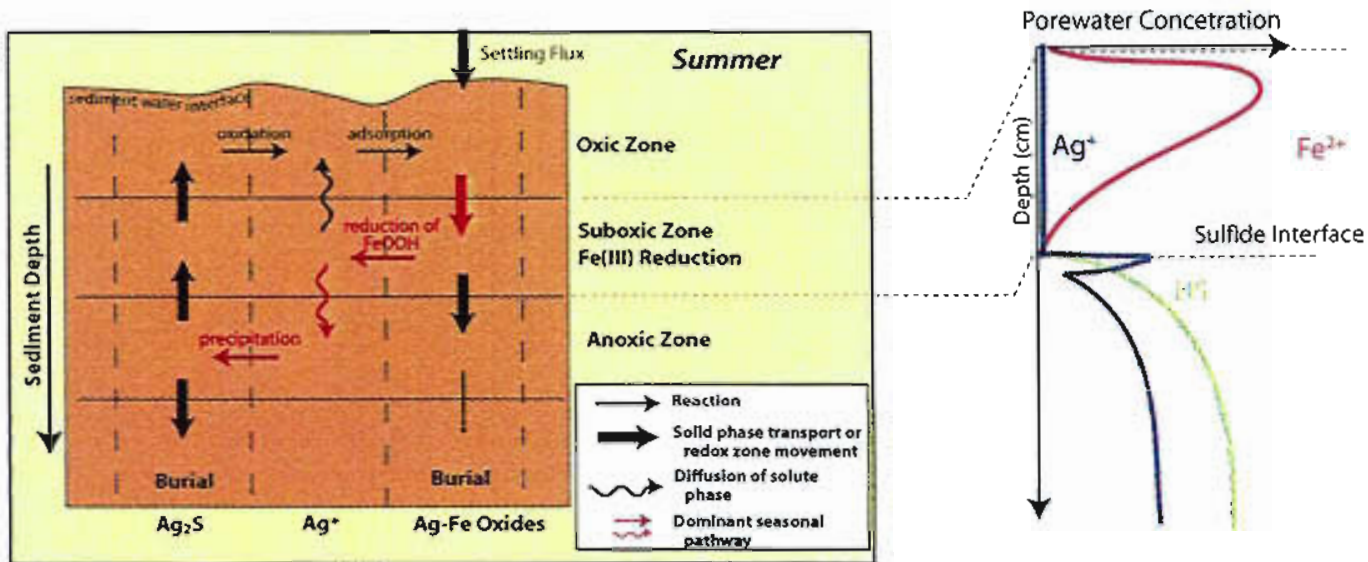

(c)
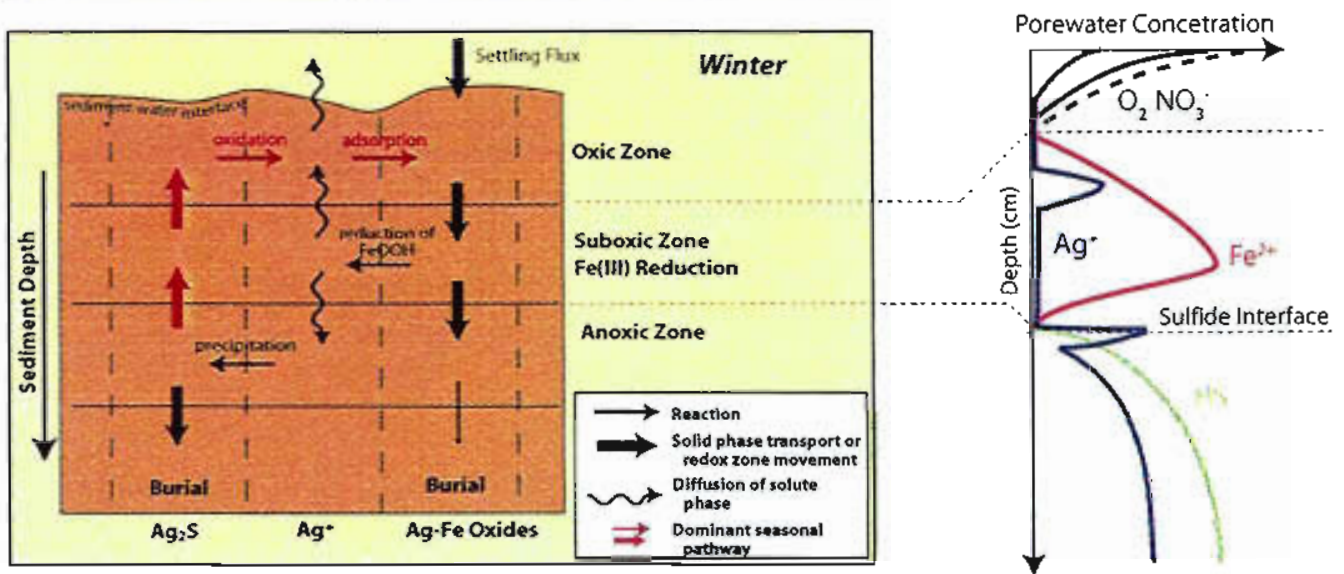

Figure 3-21: The silver cycle in Hingham Bay illustrating the dominant pathways between sediment phases in each season. Each box represents a particular sediment phase within a redox zone. The arrows represent the mechanism by which material can be transferred between boxes. Solid phase transport includes both bioturbation and sedimentation. Schematic porewater profiles are also shown. 
1980]), so from a thermodynamic point of view silver sulfide will precipitate before iron.

The silver released to the porewaters in June and July can thus precipitate out as a silver sulfide phase or altérnatively, the silver can diffuse or be mixed upwards, reach the oxic zone and be re-scavenged onto iron axyhydroxides. From the porewater profiles it should be possible to determine in which direction in which silver is diffusing. Unfortunately due to the unsteady features generated by benthic organisms this is difficult to accurately calculate. However Figure 3-22 shows the porewater profiles from June and July together with a three point moving average smoothing of the profiles. The smoothing is intended to remove some of the sharp features that, as already discussed in Section 3.3.2 have limited spatial and likely temporal extent. Also included in Figure 3-22 are the linear regression lines of the smoothed profiles used to determine the average porewater silver concentration gradients with depth, $\Delta C / \Delta z$ directed both upwards and downwards (where $C$ is the porewater concentration and $z$ is depth below the sediment-water interface). The fuxes can be calculated from Fick's First Law adapted for sediments [Berner, 1980]:

$$
J=-\phi D_{s} \frac{\partial C}{\partial z}
$$

where $J$ is the diffusion flux per area of total sediments, $\phi$ is the sediment porosity, $D_{s}$ is the whole sediment diffusion coefficient, $C$ is the concentration in the porewaters and $z$ is the depth below the sediment-water interface. The diffusion coefficient in water at infinite dilution $D$ is used to determine the whole sediment diffusion coefficient $D_{s}$ by accounting for the tortuosity, $\theta$ within the sediments.

$$
D_{s}=\frac{D}{\theta^{2}}
$$

Following the method of McDuff and Ellis [1979] a relationship between the tortuosity and porosity of the sediment is determined from resistivity and porosity measurements to derive values for $D_{s}$ for each sampling date as a function of depth. The values for $D$ for each metal are taken from Li and Gregory [1974].

The calculated average fluxes in each direction are given in Table 3.5 and it is evident that the silver released in the sub-oxic zone is removed approximately equally in both the upwards and downwards direction. Thus in late spring/early summer half the silver released to the dissolved phase that was originally associated with iron oxyhydroxides is transferred 

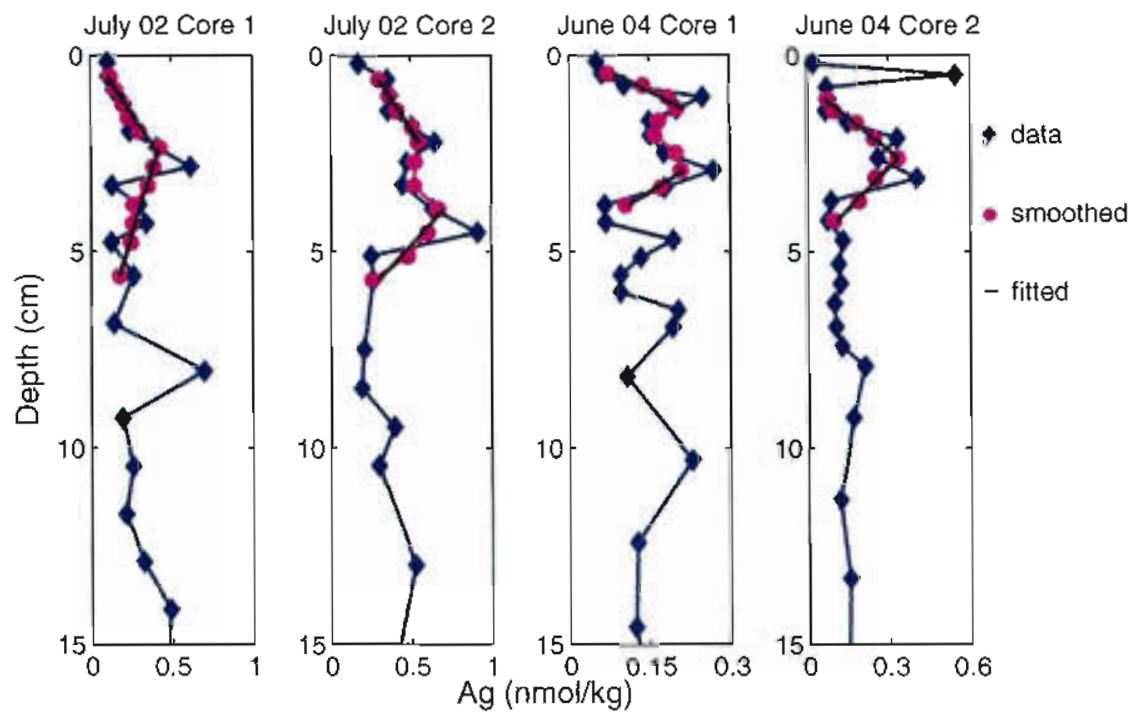

Figure 3-22: Hingham Bay porewater profile for June and July together with a three point moving average smoothing of the data. The linear regression lines used to determine porewater fluxes are also shown.

to the sulfide phase. This is the situation depicted in Figure 3-21(a).

Progressing in time from early summer, the redox horizons continue to move upwards and by September the most reducing conditions in the sediments are observed with measurable concentrations of both manganese and iron in the 0-3 mm, uppermost porewater sample. Iron oxide reduction thus occurs within millimeters of the sediment water-interface and so iron oxyhydroxides at shallower depths will have been reduced, including those which scavenged the silver released to the porewaters in late spring. The released silver, as in June and July will be transferred to either the sulfide phase or the oxide phase. Because the conditions in the sediments have become more reducing, as summer progresses a greater proportion of the released silver will be precipitated as silver sulfides rather than returned to the oxide fraction. None of the porewater profiles collected represents this situation, however it is inferred based on the differences between the July and September profiles. The September profile shows no co-incident release of silver and iron, and this must be because by September only a small fraction of silver remains associated with iron oxyhydroxides. This is illustrated in Figure 3-21(b).

The silver and iron association breaks down over summer because the dissolved iron which is also released to the porewaters is at much higher concentrations, and is more soluble with respect to sulfide. A much greater proportion of the iron is thus recycled back 
Table 3.5: Flux Calculations between the Oxic and Suboxic Zones for Silver in Hingham Bay

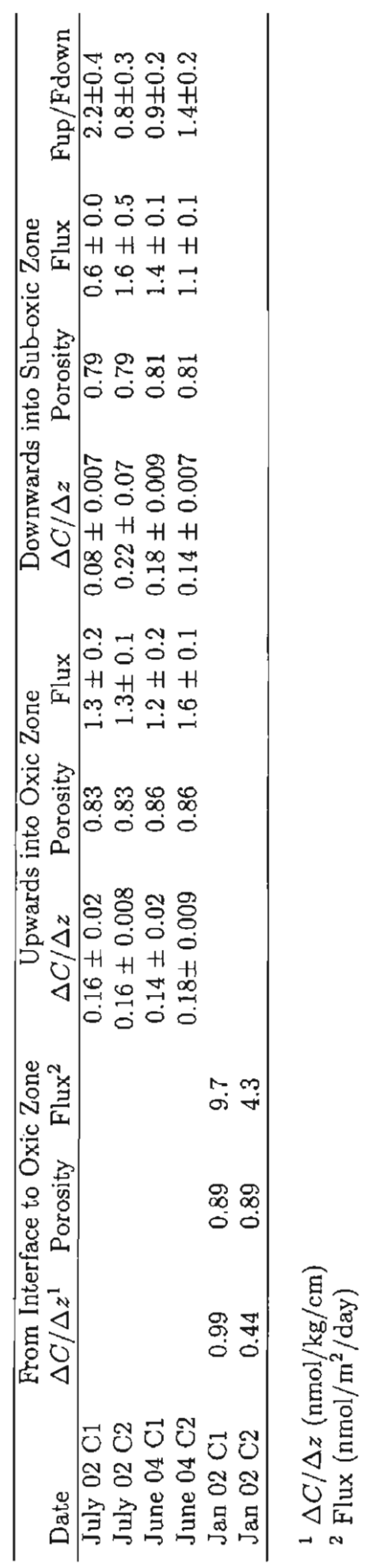


into the oxide phase. The recycled iron oxides will be reduced again (Canfield et al. [1993] found that iron could be cycled up to 300 times within a 70 day period), but over the course of the summer they will have less and less silver associated with them, and so by September the iron oxyhydroxides that are reduced appear to have completely lost all their associated silver. Thus between winter and late summer there appears to be a net transfer of mobilizable silver from oxide phases to sulfide phases.

As the temperatures start to cool and the reaction rates in the sediments slow down there is a decreased demand for oxidants and the redox horizons in the sediments move back downwards. The metals precipitated as reduced species close to the sediment-water interface can be re-oxidized as shown in Figure 3-21(c). The silver will pass through a dissolved phase before being scavenged by fresh iron oxyhydroxides. The sharp peak in silver, at $1.2 \mathrm{~cm}$, right at the front of the iron release in the January profile (Figure 3-13) may be due oxidation of silver sulfides as the redox horizon moves downwards. That there is no release of silver deeper within the zone of iron reduction is consistent with the September and October profiles and indicates that the sub-oxic conditions have persisted since the end of summer, so the iron oxides being reduced at depth have no associated silver.

In order to complete the annual cycle there must be processes operating between January and June that lead to the transfer of silver back to the iron oxyhydroxide phase so that the situation depicted in Figure 3-21(a) can be re-established. The water temperatures remain below $10^{\circ} \mathrm{C}$ until the end of April (NOAA), so the redox horizons may extend to greater depths than are observed in January. Bioturbation will be important in moving reduced species upwards. Although the magnitude of the bioturbation coefficient is less in winter, the impact of bioturbation may be greater as reduced species will be transported upwards into the oxic zone, where they will be unstable and released to the dissolved phase. The bioturbation coefficient in summer is greater, but as the sub-oxic zone is compressed closer towards the interface, bioturbation may not transport material out of the sub-oxic zone, so particles are not carried into a redox zone in which they are unstable. Any remobilized silver will be scavenged by the freshly formed iron oxyhydroxides, returning the system to the condition observed in late spring by the pathways shown in Figure 3-21(c).

There are a number of porewater peaks in the oxic and sub-oxic zones that are not explained by the conceptual model. The activity of benthic organisms can alter the local redox environment due to burrow irrigation $[$ Aller, 1980] and bioturbation can transport material 
between redox zones and thus lead to release of material that becomes thermodynamically unstable. These activities are spatially and temporally variable and so can generate peaks that do not fit within the conceptual model. An example of such a peak occurs in the October 2003 core (Figure 3-33) in the upper $1 \mathrm{~cm}$. No other porewater profiles demonstrate such a sharp release at the base of the oxic zone. Although this peak could be accounted for by oxidation of reduced phases as the redox horizon just starts to move downwards, unsteady features such as bioturbation cannot be ruled out. Sample contamination may also be responsible for this single high point.

The only strong release of silver that occurs over an extended range of depths (ie is not just a single elevated point) that does not conform to the conceptual model is the wide peak at $4-5 \mathrm{~cm}$ in Core 1 of July 2002 (Figure 3-14). This peak indicates that silver is released in the region where dissolved iron is removed from the porewaters, a feature not observed in any other profile. Examination of the solid phase data for this core (Figure 3-41) indicates there is an unusual spike with elevated silver at the same depth. This elevated solid phase silver is not observed in any other profiles and is accompanied by elevations in other trace metals such as copper and lead, iron and manganese. Although the reason behind this feature in the solid phase is unclear, the elevated solid phase levels seem to be driving the release of silver to the porewaters at this depth.

Although this conceptual model of silver and iron benthic cycling does have closure over the course of a year, it is likely that the cycle does not return to exactly the same state at the start of each year and that the cycle actually leads to the long-term redistribution of silver between oxide and sulfide phases. With variable primary productivity in the waters of Boston Harbor and Massachusetts Bay [Keller et al., 2001], annual climatic variations as well as continued sedimentation, the redox horizons will not return to exactly the same depths each year. This will lead to transfers of silver between phases over an annual cycle. The direction of net transfer is difficult to determine from the porewater data due to inadequate temporal coverage; however the solid phase data could provide a long term record of silver mobilization.

Examination of the solid phase data (Figures 3-39 to 3-41) shows no distinct evidence for a peak in solid phase silver in the surface sediments or at the top of the anoxic zone, as the porewaters might suggest. Crusius and Thomson [2003] found a very distinctive silver enrichment peak just into the anoxic zone of a turbidite due to oxic mobilization and 
subsequent trapping in the anoxic zone. However turbidite sequences are ideal locations for examination of metal redistributions due to the long-term persistence and predictability of the redox horizons and the lack of bioturbation [Thomson et al., 1993]. In sediments from the Beaufort and Chuckchi Seas, Gobeil et al. [1997] found a separation of solid phase manganese at the surface and cadmium at depth that they attributed to varying organic matter fluxes and consequent variations in the redox zone depths leading to pumping of manganese upwards and cadmium downwards. This is a similar mechanism as suggested here, except that in the Arctic it operates over much longer timescales, with lower sedimentation rate and a lack of bioturbation. This facilitates the development of an authigenic signal in the sediments. The sediments of Hingham Bay, on the other hand, exhibit seasonally varying redox horizons that can overprint past signals, bioturbation and a very high background concentration of silver that can overwhelm any diagenetic signal. Considering the fluxes into the oxic zone from the gradients shown in Figure 3-22 and the fluxes calculated from these gradients given in Table 3.5, and neglecting bioturbation and assuming one-dimensional steady state diffusion, the flux from the porewaters will equal the burial flux in the sediments:

$$
\begin{aligned}
\text { Flux }_{\text {porewater }} & =\text { Flux }_{\text {solid phase }} \\
\phi D_{s} \frac{d C}{d z} & =\omega C_{\text {authigenic }} \rho_{s}(1-\phi)
\end{aligned}
$$

The sediment burial flux of the authigenically formed metal is determined by the sediment concentration $C_{\text {authigenic }}$, the solid phase density, $\rho_{s}$ (taken as $2.65 \mathrm{~g} . \mathrm{ml}$ ) and the sedimentation rate $\omega$. The current sedimentation rate can be estimated to be $5 \mathrm{~mm} / \mathrm{yr}$. The depth of the ${ }^{137} \mathrm{Cs}$ peak in a core collected in 1978 was $35.5 \mathrm{~cm}$ [Bothner et al., 1998] and in a core collected in 2000 was $47 \mathrm{~cm}$, giving an average sedimentation rate over these years of $5 \mathrm{~mm} / \mathrm{yr}$. From this value of $\omega$, and taking a seasonally weighted average of fluxes into the oxic zone the concentration of authigenic silver can be estimated at $0.05 \mu \mathrm{mol} / \mathrm{kg}_{d r y} w t$. It is not surprising that a solid phase enrichment cannot be detected. The background concentration of silver at this depth is $30 \mu \mathrm{mol} / \mathrm{kg}_{d r y} w t$, so the authigenic fraction makes up at most $0.2 \%$ of the solid concentrations. Additionally bioturbation is a more important for solid phase transport and this will act to further obscure the authigenic signal. That 
the remobilizations indicated by the porewaters are not detected in the solid phase is thus not surprising.

The solid phase data presented here is from a total digestion, so that silver associated with non-reactive detrital phases is also measured. Evidence of a seasonal cycle and net transfer between oxic and sulfidic phases may be evident if the detrital signal can be removed from the data by performing selective extractions. A hydroxyl-amine leach and acetic acid leach [Shaw et al., 1990] can be used to identify metals associated with manganese and iron oxides and an acid volatile sulfide (AVS) analysis [Canfield, 1989] detects metals associated with non-pyritic sulfide phases. Performing these selective extractions might provide some solid phase evidence for the remobilizations evident in the porewater profiles. Indeed Cooper and Morse [1998] have observed seasonal variations in Cu in AVS fractions in Chesapeake Bay sediments.

\section{Lead and Copper Porewater Profiles}

The behavior of silver, lead and copper are all different in the oxic zone. The concentration of copper in the surface porewaters is greater than the overlying water in all cores (eg Figures 3-13 and 3-16) except June 2004 core 1 (Figure 3-18), and in all seasons except late spring/early summer (Figure 3-14) there is a strong release of copper at the sediment-water interface. This is observed in other porewater profiles from a range of oceanic and coastal environments [Klinkhammer, 1980; Elderfield et al., 1981; Sawlan and Murray, 1983; Shaw et al, 1990]. Copper is a bio-active trace metal $[S u n d a, 1994]$ and the release is attributed to the degradation of biogenic materials which contain copper.

Unlike for copper or silver there is never evidence of a release of lead at the sedimentwater interface, and the concentration of lead is consistently low throughout the oxic zone. The only exception to this is the slight elevation of lead at the interface reported in July 2002 Core 1. The concentrations of lead in the surface porewaters are usually at or below the overlying water concentrations in all cores, indicating that sometimes there may actually be a flux of lead into the sediments. Lead appears to be very strongly scavenged within the oxic zone and this maintains the porewater concentrations at low values.

Within the sub-oxic zone the lead and copper profiles resemble the silver profiles, with only some slight differences. In June and July (Figures 3-14, 3-15, 3-18 and 3-19) the release of iron to the porewaters is correlated to a release of copper and lead, suggesting that 
as with silver, these metals are associated with iron oxides and are released upon reductive dissolution of the iron oxides. Rivera-Duarte and Flegal [1994] suggested their porewater data showed similar relationships between iron and lead in San Francisco Bay. Lead scavenging by iron oxyhydroxides and release upon dissolution has been extensively studied in freshwater lake systems [Benoit and Hemond, 1990; Taillefert et al., 2000]. Copper has also been shown to associate with iron oxyhydroxides in laboratory and freshwater lake studies [Tessier et al., 1996; Guilherme and Anderson, 1998] as well as in marine porewaters [Ciceri et al., 1992]. In marine systems copper has more often been considered to associate with manganese oxides [Klinkhammer, 1980; Sawlan and Murray, 1983; Shaw et al., 1990] rather than with iron oxyhydroxides. Inspection of the manganese profiles from Hingham Bay indicate that there is no evident relationship between these two metals at this site. Hingham Bay is considerably more reducing and the concentrations of porewater iron are much higher than the open ocean sites investigated in the above studies. Higher iron oxyhydroxide concentrations and the more rapid cycling of iron at Hingham Bay will lead to a greater availability of freshly precipitated iron oxyhydroxides that may account for iron being the dominant carrier phase for copper at Hingham Bay. Other factors such as $\mathrm{pH}$, nature of the organic matter and copper speciation may also be responsible for the different sorptive behavior of copper at Hingham Bay.

The profiles do indicate that the three metals are scavenged by iron oxyhydroxides to different extents and rates. For example the release of copper at the interface is removed gradually with depth; baseline levels are reached by $3 \mathrm{~cm}$. The silver released at the interface is removed within $0.5 \mathrm{~cm}$, suggesting silver is scavenged much more rapidly than copper Results from Chapter 5 indicate that lead is scavenged onto sedimentary particles faster than either copper or silver, and the absence of a lead gradient in the surface porewaters is consistent with rapid lead scavenging.

Both the copper and lead porewater profiles are very similar to the silver porewater profiles at the sulfide interface and within the anoxic zone. Lead is a borderline soft metal [Nieboer and Richardson, 1980] and so interacts with sulfide ligands, so in the strongly sulfidic environment of the anoxic zone it is not surprising that lead behaves similarly to silver as they are both controlled by the dominant ligand. The oxidation state of copper in the presence of sulfide in environmental samples is not well known. Although $\mathrm{Cu}(\mathrm{I})$ is the thermodynamically stable form of $\mathrm{Cu}$ in anoxic conditions [Jacobs and Emerson, 
1982; Boulegue et al., 1982], it is unclear when reduction to $\mathrm{Cu}(\mathrm{I})$ occurs [Pattrick et al., 1997]. Luther et al. [2002] found $\mathrm{Cu}(\mathrm{I})$ formed in solution during the reaction of $\mathrm{Cu}(\mathrm{II})$ with sulfide prior to precipitation of CuS. However these results are from synthetic laboratory solutions and the role of organic matter in stabilizing $\mathrm{Cu}(\mathrm{II})$ is unknown. It is not possible to directly determine the oxidation state of copper in the porewaters from this dataset, however copper(I) is also a soft metal so the close correspondence between all three metals may suggest that $\mathrm{Cu}(\mathrm{I})$ is the dominant form of copper in the porewaters. Determining the isotopic ratios of copper in the porewaters may provide information on the oxidation state.

To assess if inorganic speciation alone can account for the concentrations of lead and copper measured within the anoxic zone the concentrations of total dissolved metal calculated to be in equilibrium with the sulfide minerals $\mathrm{PbS}$ (galena) and CuS (covellite) are shown in Table 3.4. Dissolved copper is calculated to be predominately $\mathrm{Cu}(\mathrm{I})$, assuraing that the sulfate-sulfide half equation is the controlling redox couple. Other mineral phases could control the free copper concentration, however $\mathrm{Cu}_{2} \mathrm{~S}$ was not considered as experiments have shown that $\mathrm{CuS}$ forms even under conditions in which $\mathrm{Cu}_{2} \mathrm{~S}$ would be expected to precipitate [Boulegue, 1983]. The $\mathrm{Cu}(\mathrm{I}) / \mathrm{Cu}(\mathrm{II})$ ratio in the dissolved phase was calculated from the sulfate-sulfide half equation and assuming the free $\mathrm{Cu}(\mathrm{II})$ concentration was determined by an equilibrium with CuS. The calculated concentrations are all lower than those measured. Although thermodynamic calculations may not be appropriate in complex sedimentary environments due to complications such as co-precipitation on FeS [Morse and Arakaki, 1993], the formation of non-stoichiometric sulfides (especially copper [Boulegue, 1983]), uncertainties in equilibrium constants and non-equilibrium conditions, this brief analysis suggests that, if simple mineral phases and inorganic ligands are controlling the lead and copper concentrations, then both these metals are over-saturated. Organic and polysulfide ligands are likely to be important [Boulegue et al., 1982; Jacobs and Emerson, 1982; Skrabal et al., 2000] in enhancing the concentrations of these metals. This is investigated in more detail in Chapter 4.

The porewater profiles suggest that lead and copper undergo the same seasonal cycling and transfer between iron oxyhydroxide phases and sulfide phases as was described for silver. The slightly differently behavior in the oxic zone, due to continuing delivery from the water column to the sediment-water interface for copper and maybe silver, as well as to differences in scavenging by iron oxides, may impact the long-term distribution of these 

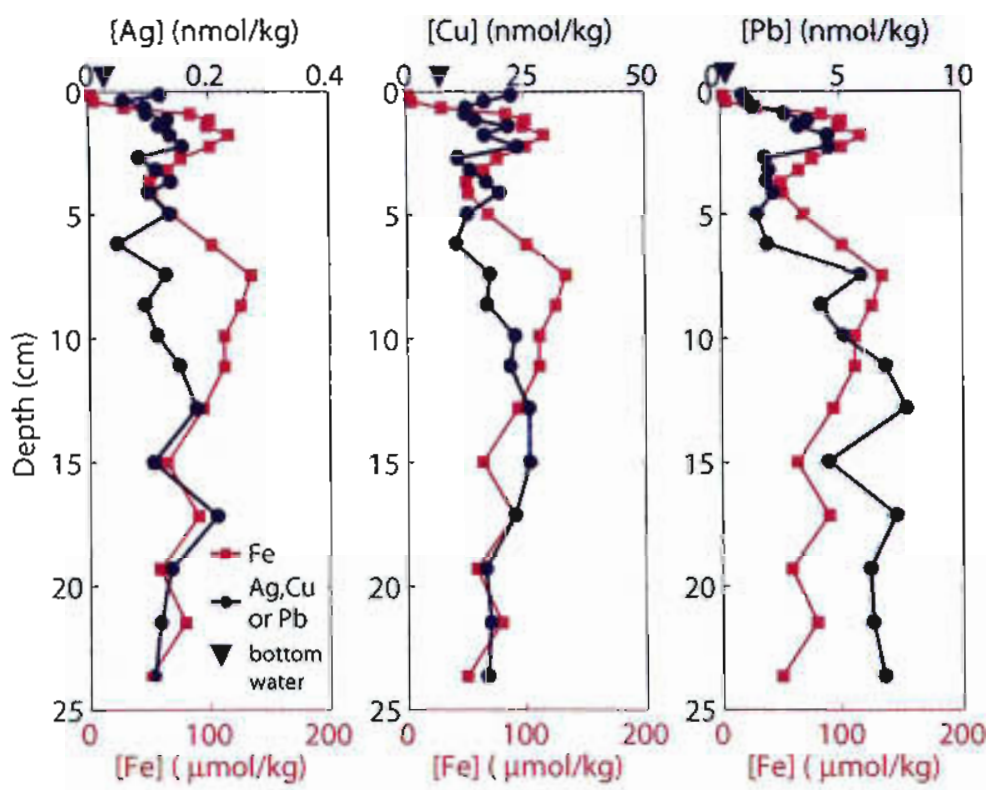

Figure 3-23: Porewater profile of silver, lead and copper for Massachusetts Bay February 2002 Core 1. Iron data is also shown on each panel.

metals. Unfortunately the high background levels in the solid phase do not enable any such differences to be distinguished.

\subsubsection{Massachusetts Bay}

The porewater profiles for silver, lead and copper at the Massachusetts Bay site are shown in Figures 3-23 to 3-25. The iron data from Figure 3-6 are also repeated in these figures. Unlike in Hingham Bay, no sulfide was detected in any core. The concentrations of silver measured in the porewaters ranged from 0.01-0.6 nmol $/ \mathrm{kg}$, and apart from a few sharp spikes are lower than those found in Hingham Bay. Despite the lower porewater concentrations the sediment partition coefficients $\log _{10} D_{A g}$ for Massachusetts Bay range from 4.3-5.0, similar to Hingham Bay, due to the correspondingly lower solid phase silver concentrations. The copper concentrations measured range from $8-43 \mathrm{nmol} / \mathrm{kg}$ and those for lead range from 0.6 $9 \mathrm{nmol} / \mathrm{kg}$. These values are comparable to those found in the Hingham Bay porewaters. The $\log _{10} D_{P b}$ for MB range between 4.6-5.5, also a similar range to that found in Hingham. Bay.

Even though worms and oxygenated burrows (with diameters up to $2.5 \mathrm{~cm}$ ) are routinely encountered while sectioning MB cores, and the iron profiles indicate a complicated 

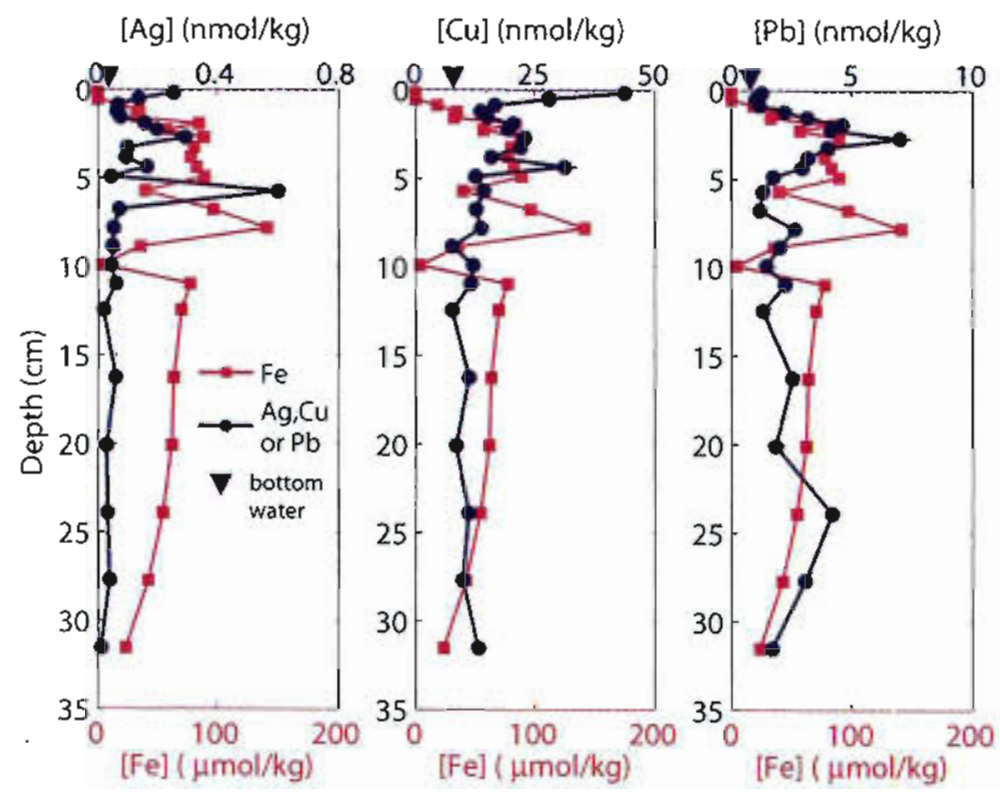

Figure 3-24: Porewater profile of silver, lead and copper for Massachusetts Bay May 2002 Core 1. Iron data is also shown on each panel.
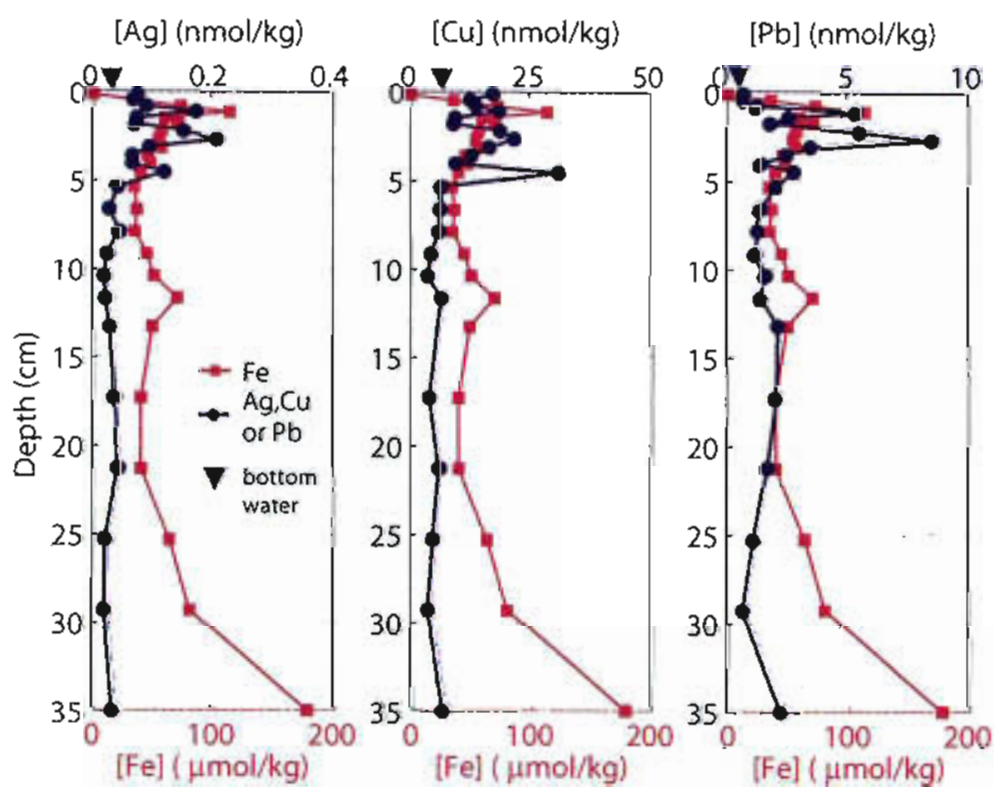

Figure 3-25: Porewater profle of silver, lead and copper for Massachusetts Bay October 2002 Core 1 . Iron data is also shown on each panel. 
diagenetic environment with non-steady state features, dominant features of the trace metal profiles for all three trace metals can easily be discerned. As in the Hingham Bay porewaters, the behavior of the metals in three depths zones can be categorized. The first zone, the oxic zone, is the zone at the surface in which there is no measurable iron in the porewaters. The second zone is the shallowest region in which dissolved iron is released and subsequently removed from the porewaters. This region extends to a depth of $3 \mathrm{~cm}$ in February 2002, $5 \mathrm{~cm}$ in May 2002 and $3 \mathrm{~cm}$ in October 2002. Zone three extends below this depth and is characterized by a secondary minimum followed by a local maximum in iron concentration, indicating iron is released to the porewaters over at least two depth ranges at this site.

The behavior of each metal within the oxic zone is the same as at the Hingham Bay site. Copper shows a strong release at the sediment-water interface throughout the year. A release of silver is only observed in the winter and late spring profiles, while a release of lead at the interface is never observed. The surface peaks in silver and copper suggest a flux to the overlying water and also into the sediments. The metals fluxing into the sediments are removed quickly with depth below the sediment-water interface.

Within the second zone, all three metals show a release coincident with the release of iron to the porewaters, suggesting that at this site too, the trace metals are scavenged by iron oxyhydroxides and are released when the oxides are reduced. The released trace metals are also removed simultaneously with iron within this zone. The removal of iron may be due to $\mathrm{Fe}_{3}\left(\mathrm{PO}_{4}\right)_{2} .8 \mathrm{H}_{2} \mathrm{O}$ precipitation and this may drive some co-precipitation of the trace metals, however a strong association between trace metals and vivianite has not been documented. There may also be trace levels of sulfide that if present will remove metals as an insoluble sulfide phase. The trace metals may also be scavenged onto other iron oxide particles that, due to the less reducing conditions at this site, exist deeper into the sediments.

Within the third zone, the concentrations of the three trace metals stabilize with depth. There are small variations in porewater concentrations in February that coincide with fluctuations in iron, but otherwise steady concentrations are reached below $7 \mathrm{~cm}$. In each core the secondary releases of iron are not accompanied by strong releases of trace metals. The release of trace metals thus coincides with the release of iron within zone 2 , but not in zone 3. Why this should be the case is not entirely clear but from Figure 3-11 there are only low levels of trace metals at this depth, and perhaps the iron oxyhydroxides that are found deeper within the sediments are less reactive and thus less likely to scavenge trace metals. 
The solid phase concentrations of trace metals in the upper $2 \mathrm{~cm}$ of the Massachusetts Bay site are elevated over the concentrations found at depth and are also higher than the concentrations measured in sediment trap data collected by the USGS (pers comm W.R. Martin). To assess if the fluxes observed in zone 2 of the porewater profiles can account for the solid phase enrichments of silver observed in Figure 3-11 the flux from the sedimentwater interface and the upward and downward fluxes from the peak associated with release of iron are calculated in Table 3.6. The same three point moving average smoothing method that was used on the Hingham Bay data was employed to determine the interior flux. The gradients selected are shown in Figure 3-26. From Table 3.6 it can be seen that the flux from the sediment-water interface dominates the porewater fluxes into the oxic zone, even though no silver flux from the surface is recorded in October. The fluxes due to release from iron oxides are of the same order of magnitude in both directions, but with the upward tlux being larger by a factor of 2 (on average). If a seasonally weighted (see Table 3.6 for weightings based on temperature) annual flux into the oxic zone is considered and Equation 3.6 is applied with an upper bound sedimentation rate for the site of $3 \mathrm{~mm} / \mathrm{yr}$ [Crusius et al., 2004 the authigenic concentration of silver can be calculated as $0.8 \mu \mathrm{mol} / \mathrm{kg}$. This is $20 \%$ of the background solids concentration. The silver solid phase data from MB indicates that the enrichment in the $0-2 \mathrm{~cm}$ surface layer is 1.4 and 1.7 times more enriched than the 5-10 cm depth zone in February and October cores respectively. The observed porewater flux thus does not account for all of the solid phase silver enrichment in the surface layer. Considering that the annual average flux was determined from just three time points this is not that surprising. A box model is developed in Section 3.3.10 to investigate the magnitude of the porewater flux that is required to explain the observed solid phase enrichment.

\subsubsection{Calculated Porewater Fluxes across the sediment-water interface}

One of the goals of this chapter is to determine the magnitude of the metal release across the sediment-water interface. From the porewater profiles the diffusive flux can be estimated with equation 3.4 and the assumption that the gradient across the sediment-water interface is one dimensional and so can be approximated as

$$
\frac{d C}{d z} \approx \frac{C_{p w 1}-C_{o l w}}{\Delta z}
$$


Table 3.6: Flux Calculations between the Oxic and Suboxic Zones for Silver in Massachusetts Bay

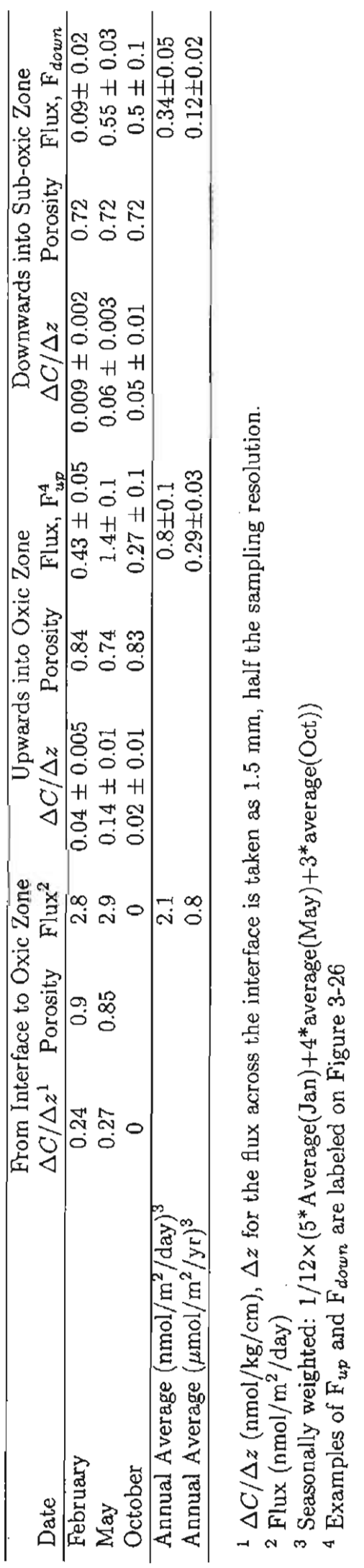



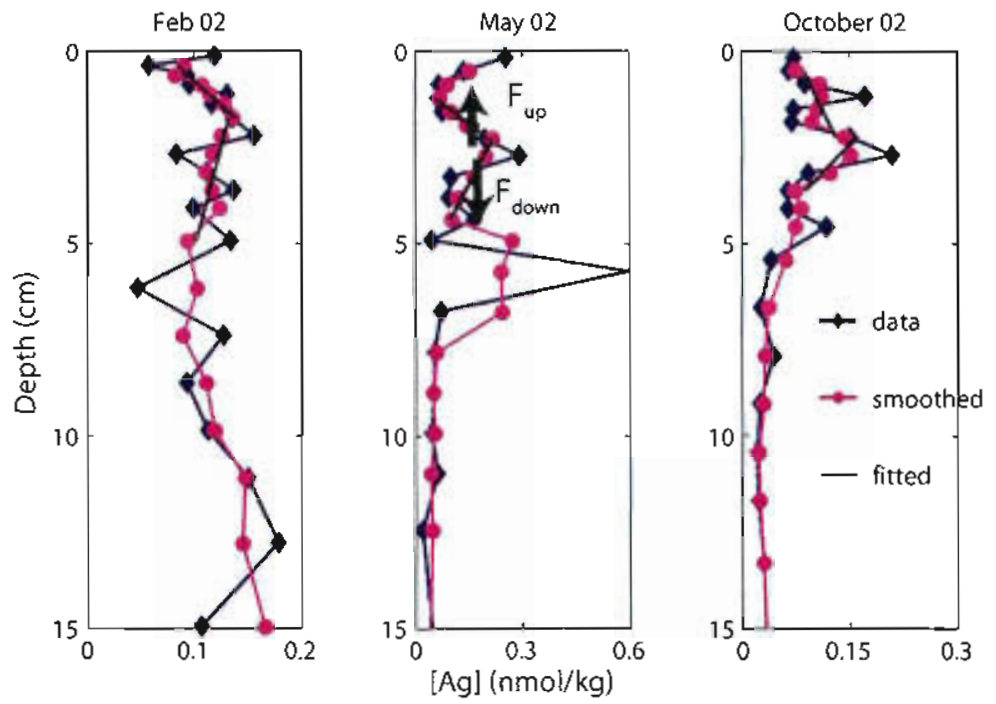

Figure 3-26: Massachusetts Bay porewater profiles together with a three point moving average smoothing of the data. The linear regression lines used to determine porewater fluxes are also shown.

where $C_{p w 1}$ is the porewater concentration in the uppermost porewater sample, that was collected over the depth range $0-z_{1} . C_{o l w}$ is the concentration measured in the water overlying the core and represents the bottom water concentration. Due to limited data resolution across the sediment-water interface, $\Delta z$ is taken as half the sampling resolution so that $\Delta z=z_{1} \times 0.5=0.15 \mathrm{~cm}$. This assumes the benthic boundary layer is narrower than $\Delta z \mathrm{~cm}$ and can be neglected. Variations in the porewater concentration within the upper $0.3 \mathrm{~cm}$ cannot be identified by the sectioning method employed and this may introduce inaccuracies into the calculated fluxes, as the $z_{1}$ driving the flux across the interface may be smaller. Davison et al. [1997] found sub-millimeter variations in the porewater distributions of metals. If a thin oxic layer persists at the sediment-water interface, even when iron is detected in the first porewater sample, this oxic layer could trap upwardly diffusing metals right at the sediment-water interface [Sundby et al., 1986; Westerlund et al., 1986]. The calculated fluxes across the sediment-water interface are shown for each core in Table 3.7. The fluxes calculated from Equation 3.7 may thus be overestimates, and so represent an upper bound for the diffusive release from the sediments. To determine an annual flux from the porewaters an annual average needs to be calculated from the data in Table 3.7. Due to only a limited number of porewater profiles available the annual average is an approximate number only. The temporal resolution of this study is considerably better than other studies which 
Table 3.7: Calculated Diffusive Flux across the sediment-water interface at Hingham Bay

\begin{tabular}{lccccc}
\hline Flux $\left(\mu \mathrm{mol} / \mathrm{m}^{2} / \mathrm{yr}\right)$ & $\mathrm{Ag}$ & $\mathrm{Pb}$ & $\mathrm{Cu}$ & $\mathrm{Fe}$ & $\mathrm{Mn}$ \\
\hline Jan 02 Core 1 & 11 & 0 & 440 & 0 & na \\
Jan 02 Core 2 & 20 & 3 & 460 & 0 & 52 \\
June 04 Core 1 & 0 & -4 & -46 & 240 & 56 \\
June 04 Core 2 & 0 & 0 & 42 & 320 & 97 \\
July 02 Core 1 & 3 & 12 & 40 & 220 & na \\
July 02 Core 2 & 2 & 16 & 36 & 260 & 170 \\
Sept 02 Core 1 & 2 & 0 & 68 & 320 & na \\
Oct 03 Core 1 & 4 & 0 & 210 & 120 & 140 \\
\hline Annual Average $\left(\mu \mathrm{mol} / \mathrm{m}^{2} / \mathrm{yr}\right)$ & 8 & 4 & 230 & 140 & 90 \\
\hline Total Flux $(\mathrm{kg} / \mathrm{yr})^{1}$ & 30 & 30 & 600 & 300 & 200 \\
\hline
\end{tabular}

${ }^{1}$ Total Flux $=$ Annual Average Flux $\times$ Sediment Area $\left(41 \mathrm{~km}^{2}\right)$

Table 3.8: Calculated Diffusive Flux across the sediment-water interface at the Massachusetts Bay site

\begin{tabular}{lccccc}
\hline Flux $\left(\mu \mathrm{mol} / \mathrm{m}^{2} / \mathrm{yr}\right)$ & $\mathrm{Ag}$ & $\mathrm{Pb}$ & $\mathrm{Cu}$ & $\mathrm{Fe}$ & $\mathrm{Mn}$ \\
Feb 02 C1 & 2 & 0 & 180 & 0 & $\mathrm{na}$ \\
May 02 C1 & 3 & 7 & 240 & 0 & $\mathrm{na}$ \\
Oct 02 C1 & 0 & 0 & 80 & 0 & 260 \\
\hline Annual Average $\left(\mu \mathrm{mol} / \mathrm{m}^{2} / \mathrm{yr}\right)$ & 2 & 2 & 180 & 0 & $\mathrm{na}$ \\
\hline Total Flux $(\mathrm{kg} / \mathrm{yr})^{1}$ & 2 & 3 & 80 & 6 & $\mathrm{na}$ \\
\hline \multicolumn{4}{l}{ Total Flux $=$ Annual Average Flux $\times$ Sediment Area $\left(7.2 \mathrm{~km}^{2}\right)$}
\end{tabular}

often use one [Rivera-Duarte and Flegal, 1997a] or two timepoints [Sundby et al., 1986; Westerlund et al., 1986] to represent the entire year. Thus the annual average calculated here does represent an improvement over past approaches. The fluxes across the sedimentwater interface for the Hingham Bay site are given in Table 3.7 and for the Massachusetts Bay site in Table 3.8 .

The fluxes from the Hingham Bay site show distinct seasonal variability. The annual silver flux is dominated by the flux in winter, and this is also the case for copper, even though there is a strong copper flux throughout the year. The lead flux is negligible for most of the year and only in July 2002 cores was the porewater concentration of lead elevated above bottorn water. The fluxes from Massachusetts Bay show reduced seasonal variability and so are more consistent throughout the year. The magnitude of the annual fluxes from Massachusetts Bay are very similar to those of Hingham Bay for lead, silver and copper. The fluxes calculated here compare in magnitude to those found in previous studies 
of porewaters. The silver fluxes found in San Francisco Bay by Rivera-Duarte and Flegal [1997a] ranged from -0.2 to $1.2 \mu \mathrm{mol} / \mathrm{m}^{2} / \mathrm{yr}$ over 9 sites, the lead fluxes ranged between 0 to $113 \mu \mathrm{mol} / \mathrm{m}^{2} / \mathrm{yr}$ [Rivera-Duarte and Flegal, 1997a] and the copper between -200 to 182 $\mu \mathrm{mol} / \mathrm{m}^{2} / \mathrm{yr}$ [Rivera-Duarte and Flegal, 1997b]. In Gullmarsfjorden Westerlund et al. [1986] measured a copper flux of $10-44 \mu \mathrm{mol} / \mathrm{m}^{2} / \mathrm{yr}$ and no detectable lead flux with a benthic chamber. The silver and copper fluxes measured here are higher than others have reported, but are still within the same order of magnitude. The porewater studies of Rivera-Duarte and Flegal have only a 1 to $2 \mathrm{~cm}$ resolution in the upper porewaters. As both copper and silver are removed rapidly over this depth range at both the Hingham and Massachusetts Bay sites the lower flux estimates for San Francisco Bay may be due their lower sampling resolution.

In order to determine the importance of the benthic fluxes of lead, silver and copper to the metal budgets of Boston Harbor and Massachusetts Bay, the fluxes determined at each site are assumed to be representative of the fine-grained regions within the Bay and Harbor. $33 \%$ or $41 \mathrm{~km}^{2}$ of Boston Harbor is fine-grained sediment, while in Massachusetts Bay only very small pockets of fine grained sediments exist, so the Massachusetts Bay site can only be considered representative of $7 \mathrm{~km}^{2}$ (pers.comm M.Bothner, USGS). These areas are used to scale up the annual average fluxes to give the basin wide estimates of the benthic fluxes, which are shown in Tables 3.7 and 3.8. These fluxes are compared in more detail to estimates of other inputs from rivers, sewage effluent and sediment resuspension in Chapter 5 (see especially Table 5.8 ). From Table 5.8 it can be seen that the diffusional flux of copper is $20 \%$ and lead is $1 \%$ of the riverine input to Boston Harbor; there are no estimates available for the riverine input of silver. Thus for copper, although the benthic diffusive flux is certainly not the major term in the metal budget, it is significant. The benthic diffusion term is a dissolved flux, while the estimates of Alber and Chan [1994] are total (dissolved + particulate) fluxes. The benthic fux is thus likely to be an even more important component of the dissolved metal budget. The benthic flux of lead is a negligible term in the lead budget, as lead seems to be so thoroughly trapped by iron oxides and other particles in the sediments that it rarely reaches a concentration in the porewaters that exceeds bottom water concentrations. Importantly, the benthic fluxes of copper and silver are likely to become even more significant terms in the budgets for these metals as continued source reduction and pollution control reduces the discharge of these metals from 
rivers and effluent sources.

\subsubsection{Benthic Chamber Data}

On selected cruises, benthic chambers were deployed in order to get a direct measurement of the fluxes across the sediment-water interface. The results for the January 2002 deployment are shown in Figure 3-27. The data from this chamber is similar to other chamber data that has been analyzed (September 2002 and June 2004) in that despite increasing concentrations of nutrients, such as ammonium there is no evident change in the concentrations of silver, lead and copper. Also shown on Figure 3-27 is the concentration that was expected in the chamber based on the fluxes calculated in Table 3.7. For silver and copper the first one or two points fall on the expected line but the concentrations fail to increase and instead remain steady over time. By the end of the chamber deployment there is a very large discrepancy between the predicted concentration and the low values measured. That the first points fall on the line may be coincidence, or it may indicate that there are scavenging processes occurring within the chamber removing metals from solution. The concentrations of lead measured in the chamber are almost constant within two standard deviations of the analytical error. All measured concentrations are considerably higher than the concentration in the overlying water, suggesting that there may be some lead contamination in the chamber. The expected flux for lead is very low, so almost no change in the lead concentration is expected and if the contamination in the first sample is taken into account, the data do roughly agree with this.

In another study comparing porewater concentrations with benthic chamber measurements of metals, Westerlund et al. [1986] found that there was no relationship between fluxes calculated from porewater profiles and the measured fluxes of cadmiuma, copper, nickel or zinc. Unlike in the Hingham Bay samples, however, these authors were expecting to see a flux into the sediments and instead measured an outward flux. They suggested that a thin oxic layer near the sediment-water interface exists where trace metal solubilization of sulfide phases takes place that their sampling resolution of $1 \mathrm{~cm}$ did not detect. As the gradients are reversed in Hingham Bay a related but opposite argument can be used to account for the lack of a measurable flux. Trapping by metal oxides in a thin oxic zone right at the sediment-water interface may be removing metals from the porewaters over depths less than $3 \mathrm{~mm}$ (the sampling resolution). If this is the case any upwardly fluxing metals are trapped 

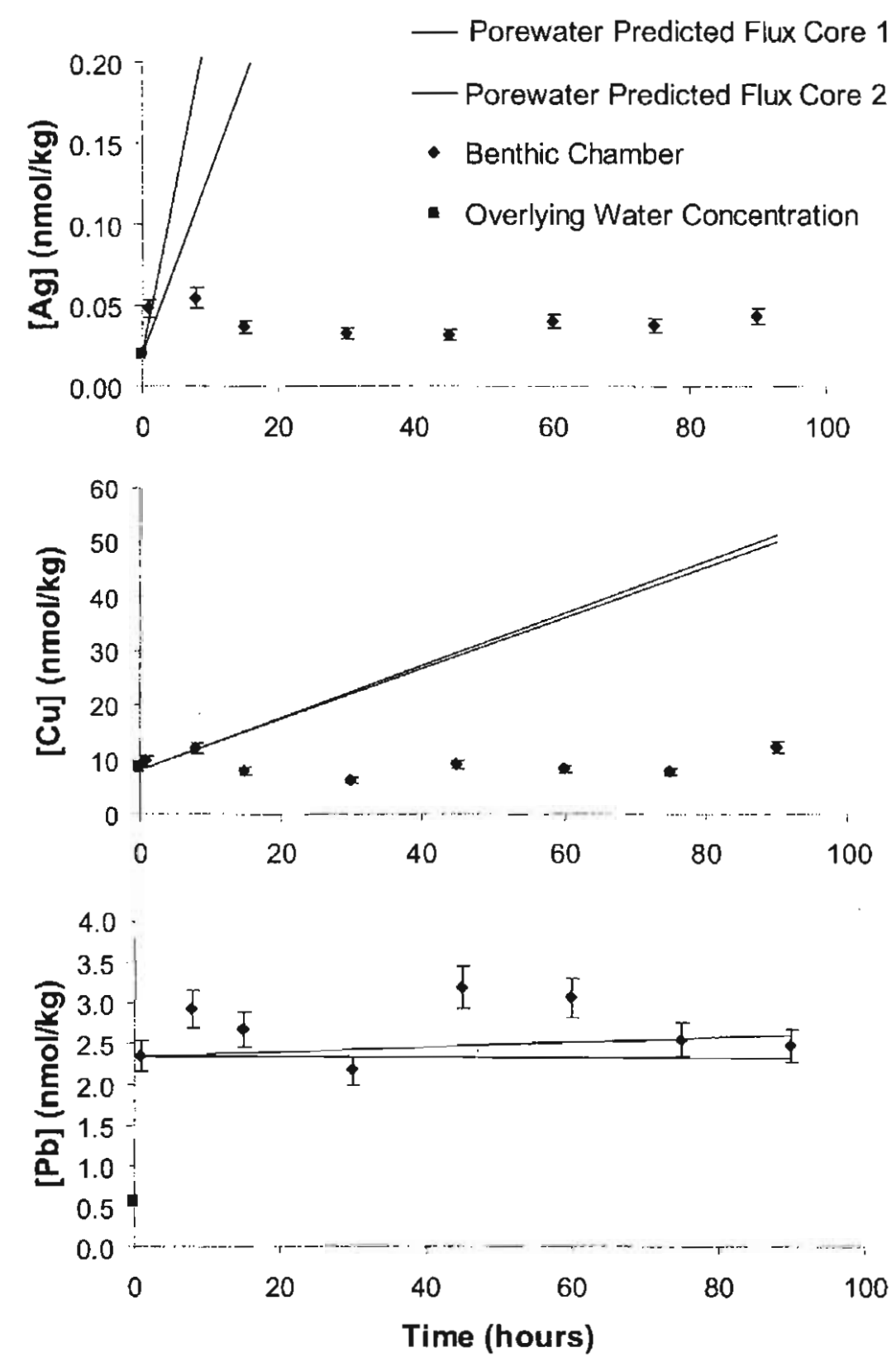

Figure 3-27: Benthic chamber data for Hingham Bay January 2002 also showing the expected concentrations calculated from the measured porewater concentration gradient across the sediment-water interface. Error bars are one standard deviation. 
within the thin upper layer but this is not identified in the porewaters. Higher resolution porewater measurements are required to assess if this is the case, and unfortunately current high resolution methods such as DET (diffusional equilibration in thin films) and electrode methods do not have sufficiently low limits of detection to be used on these metals.

Unfortunately the absence of a flux may not be due to processes occurring within the sediments but may in fact be a sampling artifact. It is difficult to get trace metal clean samples from the benthic chamber due to the metal frame supporting the chamber and the aluminosilicate glass ampules that are used to collect the samples. The lack of a trace metal flux may be due to sample poor preservation within the ampules. Disturbance of the sediment-water interface when the chamber lands may also impact trace metal samples, by stirring up small quantities of fine sediment that may stay in suspension and scavenge any released trace metals. The surfaces of the chamber itself may adsorb and remove metals from solution. The analysis of metals in samples collected by the benthic chamber was an opportunistic measurement, and the chambers were not designed specifically for trace metal sample collection, so further work may be required to make the chambers more robust for trace metal analysis. A series of laboratory tests to assess the potential for contamination or scavenging within the chamber is required before solid conclusions can be drawn from the chamber data. Iron and manganese also need to be analyzed in these samples, to see if they behave in the same way as the trace metals.

\subsubsection{Comparison Between Sites}

The seasonal cycle described for Hingham Bay, in which there is a transfer over summer of trace metals adsorbed onto iron oxyhydroxide particles to sulfide phases, does not occur at the Massachusetts Bay site. At the Massachusetts Bay site, there is release of trace metals coincident with the reduction of iron oxides in each season. In Hingham Bay this does not occur all year: in summer, when the sediments are at their most reducing, the trace metals are precipitated as sulfides. The two sites thus have very different metal cycling pathways.

Seasonal variations in porewater profiles are minimal in Massachusetts Bay, so to understand the metal cycling at this site, solid phase data are also considered. The solid phase enrichment in the surface sediments of Massachusetts Bay is the key feature that needs to be understood in order to adequately describe the metal cycling at this site. The trace metal concentration in the surface sediments is higher than the concentrations measured 


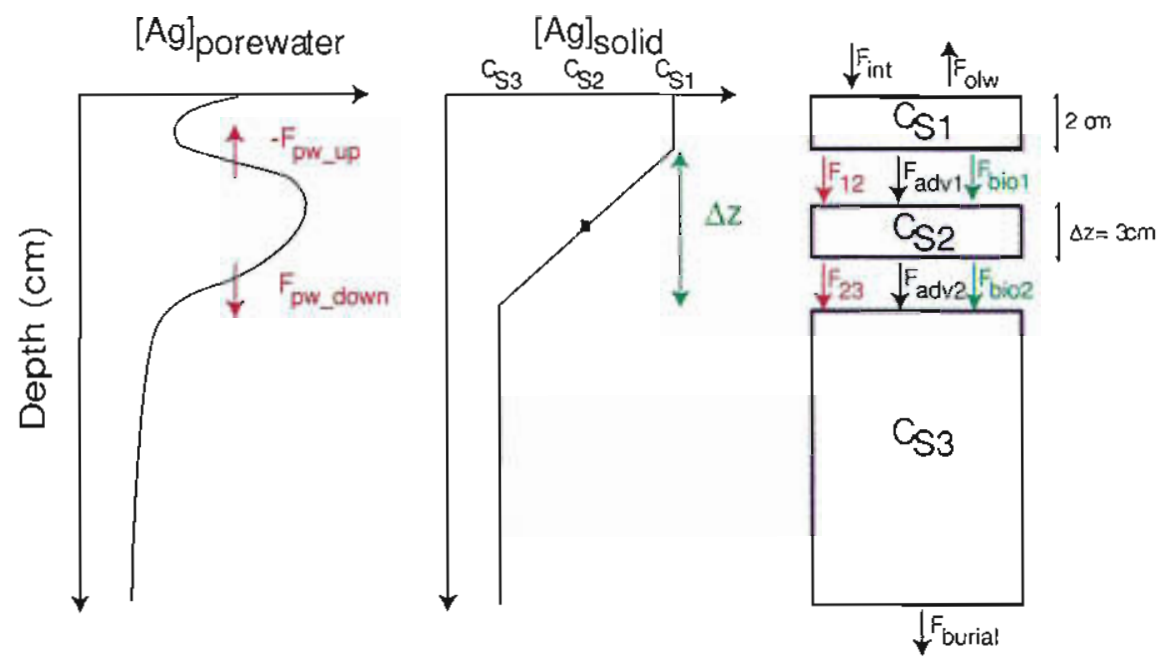

Figure 3-28: Box model for Massachusetts Bay sediment column

in sediment traps. The concentrations of organic carbon, on the other hand, is higher in the sediment traps than in the surface sediments (pers comm M. Bothner, USGS). There must thus be mechanisms active in the sediment that are enriching the surface sediments in trace metals. To quantify this in more detail a box model is developed. The model is shown in Figure 3-28 and the average porewater and solid phase profile characteristics used to define each box are also shown. This is a very simple model, designed only to capture the main features of the sediment dynamics and give some indication of the relative fluxes and their directions. The Massachusetts Bay site is compartmentalized into three boxes. The oxic zone is the upper box. Box 2 covers the first subsurface peak in dissolved iron and the lower box is Zone 3 discussed in Section 3.3.7. The solid phase concentrations within compartments 1 and 3 are largely constant with depth, so that they are well represented by a box, with average concentrations of $C_{s 1}$ and $C_{s 3}$ (in mol $/ \mathrm{kg}_{d r y w e i g h t}$ ) respectively. Box 2 is the intermediate box, and is the transition zone between the concentrations in the surface layer and deeper sediments. There is a linear solid phase profile within box 2 , so that the average concentration in box $2, C_{s 2}$ is the average of $C_{s 1}$ and $C_{s 3}$.

A mass balance over the entire sediment column assuming steady state gives

$$
F_{\text {interface }}=F_{\text {olw }}+F_{\text {burial }}
$$

where $F_{\text {interface }}$ is the depositional flux at the sediment-water interface, $F_{\text {olw }}$ is the flux out 
of the sediments to the overlying water, due to all possible mechanism such as diffusion, irrigation and sediment resuspension, and $F_{\text {burial }}$ is the burial flux, which is given by

$$
F_{\text {burial }}=\left(1-\phi_{\infty}\right) \rho_{s} \omega_{\infty} C_{s \infty}
$$

where $\omega$ is the sedimentation rate, $\rho_{s}$ is the solid phase density (taken to be $2.65 \mathrm{~g} / \mathrm{ml}$ ) and the subscript $\infty$ indicates the values found at a depth within the sediments that can be assumed to be permanently buried on an early diagenetic timescale. A constant mass flux is assumed, so that;

$$
\omega_{\infty} \rho_{s}\left(1-\phi_{\infty}\right)=\omega_{z} \rho_{s}\left(1-\phi_{z}\right)
$$

where $z$ is any depth within the sediments. The solid phase profiles within the depth range encompassed by the lower box in Massachusetts Bay are largely constant so the burial flux can be well approximated as;

$$
F_{\text {burial }}=\left(1-\phi_{3}\right) \rho_{s} \omega_{3} C_{s 3}
$$

Assuming that each individual box is also at steady state, a mass balance for the oxic zone box, box 1 , gives that

$$
F_{\text {inter face }}=F_{o l w}+F_{12}+F_{a d v 1}+F_{b i o t u r b 1}
$$

where $F_{12}$ is a flux between box 1 and box 2 , and includes, diffusive + irrigation fluxes out of the oxic zone, $F_{a d v 1}$ is the solid phase flux out of the oxic zone due to sedimentation and $F_{\text {bioturbl }}$ is the bioturbation flux between box 1 and 2 due to mixing by organisms. Similarly a mass balance for box 2 gives that

$$
F_{12}+F_{a d v 1}+F_{b i o t u r b 1}=F_{23}+F_{a d v 2}+F_{b i o t u r b 2}
$$

where $F_{21}$ is a flux between box 2 and box $3, F_{a d v 2}$ is the solid phase flux out box 2 due to sedimentation and $F_{\text {bioturb2 }}$ is the bioturbation flux between the box 2 and 3 due to mixing by organisms. Finally the mass balance over box 3 gives

$$
F_{\text {burial }}=F_{23}+F_{a d v 2}+F_{b i o t u r b 2}
$$


The advective and bioturbation terms out of box $i$ are given below, with the assumption that the bioturbation flux can be described as a diffusional process with a biodiffusion coefficient of $D_{b}$;

$$
\begin{aligned}
& F_{\text {adv } i}=\left(1-\phi_{i}\right) \rho_{s} \omega_{i} C_{s i} \\
& F_{\text {bioturb } i} \simeq\left(1-\phi_{i}\right) \rho_{s} D_{b} \frac{C_{s i}-C_{s(i+1)}}{\Delta z / 2}
\end{aligned}
$$

The depth $\Delta z$ taken for the box model is shown in Figure 3-28 and is the thickness of box 2 , the transition zone between the surface and deeper concentrations. The biodiffusion coefficient is assumed to be constant over $\Delta z$. The ${ }^{234} T h$ profiles only provide data for the upper $2 \mathrm{~cm}$ or so, so this assumption requires some extrapolation and may overestimate the bioturbation flux. From the porewater profiles, there is a porewater flux of silver upwards into the oxic box $-F_{p w_{u} p}$ and downwards into the lower box, $F_{p w_{d} o w n}$ and these contribute to $F_{12}$ and $F_{23}$ respectively. Combining Equations 3.8, 3.10 and 3.12 with the expressions for the advection and bioturbation terms gives an expression for the flux $F_{12}$ that is required to maintain the measured concentrations in the solid phase:

$$
F_{12}=\rho_{s}(1-\phi)\left[\omega\left(C_{s 3}-C_{s 1}\right)+\frac{D_{b}}{(\Delta z / 2)}\left(C_{s 2}-C_{s 1}\right)\right]
$$

$C_{\mathrm{s} 2}$ is the average of $C_{s 1}$ and $C_{s 3}$, so that Equation 3.17 can be simplified to give:

$$
F_{12}=\left(C_{s 3}-C_{s 1}\right) \rho_{s}(1-\phi)\left(\omega+D_{b} / \Delta z\right)
$$

A comparison of the magnitudes of $F_{12}$ and $F_{23}$ can be obtained from Equation 3.13 to give

$$
F_{12}-F_{23}=\rho_{s}(1-\phi) \omega\left(C_{3}-C_{1}\right) / 2
$$

Equation 3.18 indicates that the direction of the flux, $F_{12}$ controls whether the surface box is enriched relative to the lower box. For a surface enrichment, as found in Massachusetts Bay, there must be an upward flux. The magnitude of the enrichment is determined by the sedimentation rate and intensity of bioturbation $\left(D_{b} / \Delta z\right)$. This is shown graphically in Figure 3-29. From this Figure it can be seen that the higher the bioturbation intensity or sedimentation rate for a given $F_{12}$ the smaller the enrichment $\left(\mathrm{C}_{s 3}-\mathrm{C}_{s 1}\right)$ will be. A 


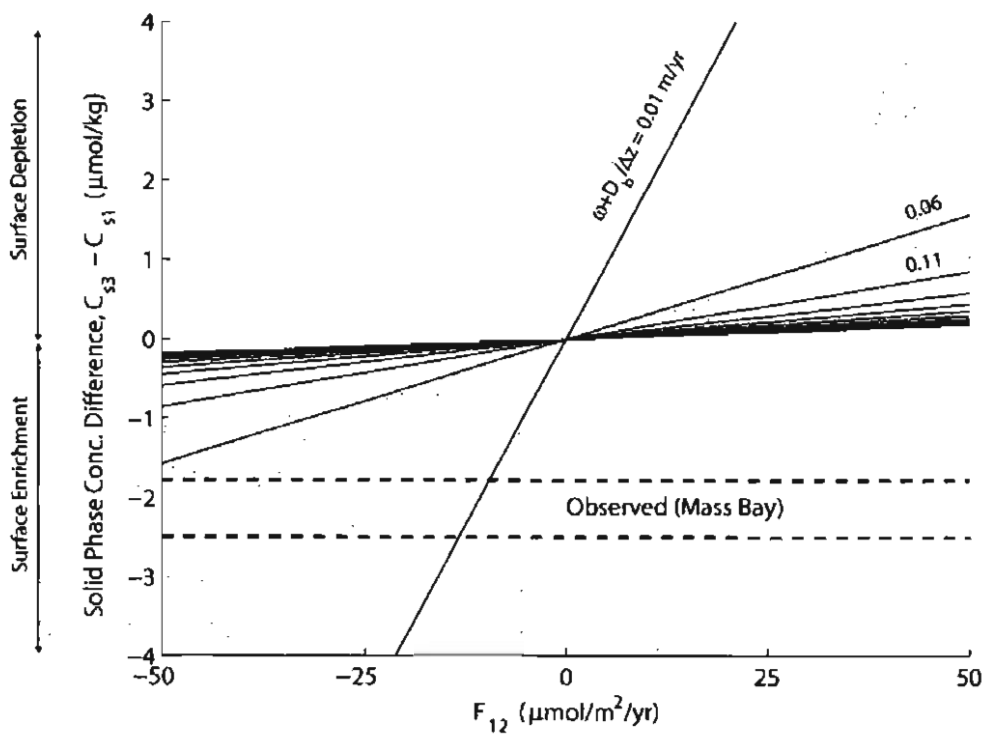

Figure 3-29: Solid phase concentration distribution generated by a range of $F_{12}$ fluxes. The black lines represent values of $\omega+D_{b} / \Delta z$ ranging from 0.01 to $0.5 \mathrm{~m} / \mathrm{yr}$. The shaded areas represent the range of possible $\omega+D_{b} / \Delta z$ values for the Massachusetts Bay site with values of $\omega$ ranging from 1 to $3 \mathrm{~mm} / \mathrm{yr}$ and $D_{b}$ ranging from 5 to $27 \mathrm{~cm}^{2} / \mathrm{yr}$. The observed concentration differences between the surface and deeper sediments are shown as dashed lines

large enrichment cannot develop if the bioturbation intensity is high, as any species that diffuses upwards is mixed back downwards, or if the sedimentation rate is high, so that the species is moved out of the surface box before a large concentration can build up. At low values of $\omega+D_{b} / \Delta z$ the level of enrichment is particularly sensitive to changes in either the sedimentation rate or bioturbation intensity.

Figure 3-29 indicates that for the case where there is a surface phase depletion, the bioturbation and sedimentation rate control the magnitude of the depletion in the same manner. For there to be a surface depletion there must be a net downwards flux. A surface depletion as observed in the Arctic Seas by Gobeil et al. [1997], where cadmium concentrations in the upper $10 \mathrm{~cm}$ of the sediment column are depleted by up to $1.5 \mu \mathrm{g} / \mathrm{g}$ relative to the concentrations at depth. In the Arctic Seas the net downward flux is driven by oscillations in the redox zones pumping cadmium downwards to the sulfide phase Gobeil et al. [1997].

In Massachusetts Bay from excess ${ }^{234} T h$ profiles the biodiffusion coefficient was determined to be $5 \mathrm{~cm}^{2} \mathrm{yr}^{-1}$ in both February 2002 and September 2003 and was $27 \mathrm{~cm}^{2} \mathrm{yr}^{-1}$ 
in May 2002 (pers comm W. Martin). Crusius et al. [2004] suggest that an upper bound for the sedimentation rate in Massachusetts Bay is $3 \mathrm{~mm} / \mathrm{yr}$ and for comparison purposes a lower bound of $1 \mathrm{~mm} / \mathrm{yr}$ is also included. The value of $\Delta z$ is taken to be $3 \mathrm{~cm}$, so that the range of $\omega+D_{b} / \Delta z$ possible at the Massachusetts Bay site is 0.018 to $0.09 \mathrm{~m} / \mathrm{yr}$. These values are plotted as the shaded region in Figure 3-29. From the observed values of $C_{s 3}-C_{s 1}$ plotted on Figure 3-29 it can be seen that given the possible range in $\omega+D_{b} / \Delta z$, an upward $F_{12}$ flux between 15 and $50 \mu \mathrm{mol} / \mathrm{m}^{2} / \mathrm{yr}$ is required to maintain the observed solid phase profile.

To investigate the relative magnitudes of each flux term, average values are used to calculate each flux. For solid phase concentrations of $C_{s 1}=6.3 \mu \mathrm{mol} / \mathrm{kg}$ and $C_{s 3}=4.4 \mu \mathrm{mol} / \mathrm{kg}$, a seasonal averaged value for $D_{b}$ of $10 \mathrm{~cm}^{2} \mathrm{yr}^{-1}$ and a sedimentation rate of $1 \mathrm{~mm} / \mathrm{yr}$ gives the fluxes shown in Table 3.9. It is evident that the upwardly directed flux $F_{12}$ is largely balancing out the downwardly directed bioturbation flux, and this is the case even at lower values of $D_{b} . F_{12}$ is just slightly higher than the $F_{23}$, indicating that this flux originates from box 3 , and from a mass balance point of view, moves through box 2 largely unaltered, with just a small addition within box 2 to balance advection terms. The diffusive flux across the sediment-water interface, given in Table 3.8 is used to estimate $F_{\text {olw }}$ in Table 3.9 and this would indicate that $70 \%$ of material arriving at the sediment-water interface is ultimately buried. $F_{o l w}$ is probably underestimated as it does not include remobilization due to sediment resuspension, so this is an upper estimate of the burial efficiency.

Comparing the calculated flux $F_{12}$ at Massachusetts Bay to the measured diffusive porewater fluxes given in Table 3.6 indicates that the annual-average diffusive porewater flux into the oxic zone is $0.3 \pm 0.03 \mu \mathrm{mol} / \mathrm{m}^{2} / \mathrm{yr}$. This is considerably lower than the estimated range from Figure 3-29 of $F_{12}=15-50 \mu \mathrm{mol} / \mathrm{m}^{2} / \mathrm{yr}$. In addition the porewater profiles indicate there is a diffusive flux downwards from box 2 to box 3 of $0.1 \pm 0.02 \mu \mathrm{mol} / \mathrm{m}^{2} / \mathrm{yr}$, while the box model suggests $F_{23}$ is also directed upwards. Diffusive fluxes are thus only a small fraction of $F_{12}$ and $F_{23}$. Irrigation fluxes may be contributing to the flux of material from box 3 to box 1 . This could come about as oxygenated water drawn into a burrow that extends into the suboxic zone, could oxidize silver sulfides. As the irrigating water is expelled, the dissolved silver may be transported out of the suboxic zone but be trapped by adsorption to iron oxides. lining the burrow wall within the oxic zone.

As $F_{12}$ and $F_{23}$ balance all the other fluxes, if any of these fluxes are inaccurately 
Table 3.9: Flux terms calculated for the Massachusetts Bay site

\begin{tabular}{lc}
\hline Calculated Flux & $\left(\mu \mathrm{mol} \mathrm{m}^{-2} / \mathrm{yr}\right)$ \\
\hline Box 1 & 2 \\
$F_{\text {olw }}$ & 7 \\
$\mathrm{~F}_{\text {interface }}$ & 7 \\
$\mathrm{~F}_{\text {adv1 }}$ & 27 \\
$\mathrm{~F}_{\text {tioturb1 }}$ & -29 \\
$\mathrm{~F}_{12}$ & \\
Box 2 & 6 \\
$\mathrm{~F}_{\text {adv2 }}$ & 27 \\
$\mathrm{~F}_{\text {bioturb2 }}$ & -28 \\
$\mathrm{~F}_{23}$ & \\
Box 3 & \\
$\mathrm{F}_{\text {burial }}$ & 5 \\
\hline
\end{tabular}

$D_{b}=10 \mathrm{~cm}^{2} / \mathrm{yr}, \omega=1 \mathrm{~mm} / \mathrm{yr}$ $\phi_{1}=0.84, \phi_{2}=0.58, \Delta z=3 \mathrm{~cm}$

1 from Table 3.8

estimated, this will impact the magnitude of these two unknown fluxes. The bioturbation flux was determined using total-extraction solid phase data. This is a simplification of the situation, as in reality bioturbation will mix oxidized solid phases downwards (as they have a higher concentration close to the surface) and mix reduced solid phases upwards (as they have a higher concentration deeper within the sediments). It is thus possible that a portion of $F_{12}$ and $F_{23}$ is also due to upward mixing of reduced solids. Further solid phase analysis and selective extractions will enable better estimates of the bioturbation fluxes, and allow this hypothesis to be tested.

The upward $F_{12}$ flux that the box model predicts gives insights into the metal cycling at the Massachusetts Bay site. When particulate silver is moved into the sub-oxic zone, by either downwards mixing or by burial, reduction of iron oxides releases adsorbed silver to the porewaters. In order to maintain an upwards flux more of the dissolved silver must flux upwards, where it will be re-absorbed just above the suboxic zone by iron oxides (and can thus be released due to iron reduction again). An oxide scavenging-release due to reduction- oxide scavenging loop must thus exist. For this to be the case iron oxides must be a stronger sink of silver than alternative solid phases below. Iron seems to be removed within the suboxic zone by precipitation as a phosphate mineral, vivianite. As silver is a soft metal it is unlikely to strongly associate with vivianite. Precipitation as a silver sulfide phase at depth due to the presence of trace levels of sulfide is likely, but sulfide 
precipitation must be less significant than the transfer to the oxide sink. When reduced solid phases are mixed upwards into the oxic zone, oxidation and subsequent scavenging by iron oxides will also lead to a transfer of material to the iron-oxide phase, so further enhancing the surface enrichment. This is the key difference between the Massachusetts Bay site and the Hingham Bay site. In late spring and summer in Hingham Bay most of the silver is removed downwards to the sulfide phase.

It is not accurate to apply a steady state model to the sediments of Hingham Bay. The solid phase distribution for Hingham Bay is also shown in Figure 3-28 and it is obviously quite different from that of Massachusetts Bay. Due to the historical high loadings of metals to the site, that only ceased in 1990 , the sediments cannot be considered at steady state. In particular Equation 3.8 does not apply as the burial flux is determined by historical contamination levels and is not balanced by the current diagenetic processes in the upper sediment column. Due to the temporal variability in metal loadings, as well as sedimentation rate a more complex model that does not require a steady state assumption is required to adequately describe this site. A finite difference model solving the general diagenetic equations [Bemer, 1980] with coupled solid and dissolved phases may be necessary. Using the model to determine if over the course of a seasonal cycle there is a net downward or upward porewater flux would be especially interesting as this result will have important impacts on the prediction of the long term distribution of metals in the sediments at this site. Unfortunately this would involve considerably more effort and is beyond the scope of this chapter.

\subsubsection{Conclusions}

The seasonal cycle of silver, lead and copper in the sediments of two contrasting coastal sites has been investigated. At both sites due to the adsorption of these trace metals onto iron oxyhydroxides there is a strong coupling between the iron redox cycle and the metal behavior. The reduction of iron axyhydroxides leads to the release of dissolved metals to the porewaters. The released trace metals are removed from solution by either diffusing upwards and readsorbing to iron oxyhydroxides or diffusing downwards and precipitating as insoluble sulfide phases. At each site due to different redox conditions the metal cycles are quite different.

The furst site is in Hingham Bay, Boston Harbor and is highly contaminated and a 
strongly reducing site with sulfidic conditions $8 \mathrm{~cm}$ below the sediment-water interface. There is a distinct seasonal oscillation in the depth of the redox zones that has a large impact on the metal cycle. The porewater profiles indicate that in late spring the metals remobilized by the reduction of iron are transferred equally in both directions. As the conditions become more reducing over summer, and increased sulfate reduction leads to higher sulfide concentrations, more of the metals released to the porewaters are removed downwards and precipitated as sulfide phases. By the end of summer all the trace metals formerly associated with iron oxyhydroxides are transferred to the sulfide phase, so that the porewater profiles suggest no relationship between the iron cycle and the trace metal cycle at this time. As the conditions cool and the redox horizons move back downwards the sulfide phases are re-oxidized and the metals are transferred back to freshly formed iron oxyhydroxides to complete the annual cycle.

The second site is further offshore in Massachusetts Bay. This site is less reducing than the Hingham Bay site and sulfide is not detected in the upper $40 \mathrm{~cm}$ of sediment. There is not such a distinct seasonal oscillation of the redox zones, and a release of trace metals coincident with the reduction of iron oxyhydroxides is observed throughout the year. There is a surface layer at this site enriched in iron, manganese and other trace metals, including silver, lead and copper. To support this enrichment there must be a net flux of metals upwards. The preferential removal of metals upwards is because the Massachusetts Bay site is less reducing and so the strong sulfide sink does not exist. At Massachusetts Bay the metal cycling is such that the trace metals are focussed into the enriched layer at the surface.

The differences between the two sites illustrate the different pathways of metal cycling that are possible within the coastal zone. In strongly reducing sediments with measurable sulfide close to the sediment-water interface, trace metals could be preferentially pumped downwards and immobilized as sulfides. In sediments where sulfide is not detected, the metals will be focused close to the sediment-water interface due to preferential scavenging by metal oxides. This difference in cycling is particularly important to understand for the long term management of contaminated sites. The consequences of contamination will be greater at less reducing sites as the contamination at the surface will persist because the metals will be focused close to the sediment-water interface by diagenetic processes rather than being buried. 
The fluxes of metals calculated from the porewater profiles at each site indicate that there is a flux of copper from the sediments throughout the year, while silver shows release at the sediment-water interface only between winter and spring. Lead is strongly scavenged and has negligible release to the overlying waters. The diffusive flux of copper from the sediments is $20 \%$ of the copper input due to rivers and so is an important term in the metal budgets of Boston Harbor. The benthic release of both silver and copper are likely to become more significant terms in the metal budget for Boston Harbor as pollution control continues to reduce the discharge of metals to the environment from other sources. 
3.4 Compilation of Porewater Data 
Table 3.10: Porewater Data

\begin{tabular}{|c|c|c|c|c|c|c|c|}
\hline & $\begin{array}{l}\text { Mid-Depth } \\
\text { (cm) }\end{array}$ & $\begin{array}{c}{[\mathrm{Ag}]} \\
(\mathrm{nmol} / \mathrm{kg})\end{array}$ & $\begin{array}{c}{[\mathrm{Pb}]} \\
(\mathrm{nmol} / \mathrm{kg})\end{array}$ & $\begin{array}{c}{[\mathrm{Cu}]} \\
(\mathrm{nmol} / \mathrm{kg})\end{array}$ & $\begin{array}{c}{[\mathrm{Fe}]} \\
(\mu \mathrm{mol} / \mathrm{kg})\end{array}$ & $\begin{array}{c}{[\mathrm{Mn}]} \\
(\mu \mathrm{mol} / \mathrm{kg})\end{array}$ & $\begin{array}{c}{[\mathrm{S}]} \\
(\mu \mathrm{mol} / \mathrm{L})\end{array}$ \\
\hline HB0102c1 & 0.1 & 0.41 & 0.5 & 42.4 & 0 & & 0 \\
\hline HB0102c1 & 0.4 & 0.35 & 0.7 & 40.4 & 0 & & 0 \\
\hline HB0102c1 & 0.7 & 0.16 & 1.0 & 18.4 & 35 & & 0 \\
\hline HB0102c1 & 1.0 & 0.34 & 3.8 & 39.5 & 115 & & 0 \\
\hline $\mathrm{HB} 0102 \mathrm{c} 1$ & 1.2 & 0.31 & 3.8 & 24.8 & 392 & & 0 \\
\hline HB0102c1 & 1.5 & 0.23 & 2.3 & 14.3 & 210 & & 0 \\
\hline HB0102c1 & 1.9 & 0.14 & 1.7 & 31.8 & 234 & & 0 \\
\hline $\mathrm{HB} 0102 \mathrm{c} 1$ & 2.4 & 0.06 & 1.1 & 7.2 & 219 & & 0 \\
\hline НВ0102c1 & 2.8 & 0.05 & 0.9 & 6.3 & 154 & & 0 \\
\hline HB0102c1 & 3.3 & 0.04 & 0.7 . & 7.0 & 111 & & 0 \\
\hline HB0102c1 & 3.8 & 0.04 & 0.7 & 5.5 & 97 & & 0 \\
\hline HB0102c1 & 4.3 & 0.07 & 0.9 & 6.0 & 87 & & 0 \\
\hline HB0102c1 & 5.0 & 0.05 & 0.8 & 8.2 & 100 & & 6 \\
\hline HB0102cl & 6.0 & 0.04 & 0.7 & 8.8 & 21 & & 12 \\
\hline HB0102c1 & 7.0 & 0.10 & 0.9 & 16.7 & 2 & & 120 \\
\hline HB0102c1 & 8.0 & 0.12 & 24.1 & 12.6 & 2 & & 165 \\
\hline HB0102c1 & 9.0 & 0.12 & 2.1 & 12.7 & 0 & & 410 \\
\hline HB0102c1 & 10.0 & 0.16 & 1.9 & 10.9 & 0 & & 640 \\
\hline HB0102c1 & 11.5 . & 0.25 & 13.4 & 20.9 & 0 & & 595 \\
\hline HB0102c1 & 13.5 & 0.22 & 2.7 & 13.9 & 0 & & 943 \\
\hline HB0102c1 & 15.5 & 0.19 & 4.0 & 13.9 & 0 & & 1050 \\
\hline HB0102cl & $17.5^{\prime}$ & 0.16 & 10.7 & 16.8 & 0 & & 1008 \\
\hline HB0102c1 & 19.5 & 0.17 & 2.6 & 13.3 & 0 & & 926 \\
\hline HB0102c1 & 21.5 & 0.07 & 3.3 & 9.6 & 0 & & 804 \\
\hline HB0702c1 & 0.2 & 0.12 & 1.9 & 11.9 & 29 & 20 & 0 \\
\hline HB0702c1 & 0.5 & 0.12 & 0.5 & 9.0 & 28 & 17 & 0 \\
\hline HB0702c1 & 0.9 & 0.15 & 1.0 & 13.0 & 76 & 14 & 0 \\
\hline HB0702c1 & 1.2 & 0.22 & 1.4 & 11.3 & 87 & 10 & 0 \\
\hline HB0702c1 & 1.6 & 0.25 & 2.5 & 15.8 & 104 & 10 & 0 \\
\hline HB $0702 c 1$ & 1.9 & 0.25 & 1.9 & 13.0 & 109 & 6 & 0 \\
\hline HB0702c1 & 2.3 & 0.42 & 4.4 & 22.9 & 136 & 3 & 0 \\
\hline HB0702c1 & 2.8 & 0.63 & 6.4 & 29.8 & 168 & 5 & 0 \\
\hline HB0702c1 & 3.3 & 0.14 & 1.5 & 9.2 & 104 & 5 & 0 \\
\hline $\mathrm{HB} 0702 \mathrm{cl}$ & 3.8 & 0.32 & 1.3 & 9.0 & 139 & 0 & 0 \\
\hline HB0702c1 & 4.3 & 0.35 & 4.2 & 21.5 & 216 & 5 & 0 \\
\hline HB0702c1 & 4.8 & 0.14 & 1.6 & 10.2 & 85 & 7 & 0 \\
\hline HB0702c1 & 5.6 & 0.27 & 2.9 & 17.1 & 164 & 4 & 0 \\
\hline $\mathrm{HB} 0702 \mathrm{c} 1$ & 6.8 & 0.15 & 1.3 & 9.7 & 12 & 2 & 0 \\
\hline $\mathrm{HB} 0702 \mathrm{c} 1$ & 8.0 & 0.71 & 11.0 & 31.2 & 5 & 0 & 55 \\
\hline НB0702c1 & 9.2 & 0.21 & 2.2 & 14.5 & 0 & 2 & 373 \\
\hline $\mathrm{HB} 0702 \mathrm{cl}$ & 10.5 & 0.27 & 2.9 & 15.1 & 0 & 0 & 516 \\
\hline $\mathrm{HB} 0702 \mathrm{cl}$ & 11.7 & 0.23 & 2.4 & 14.2 & 0 & 1 & 811 \\
\hline HB0702c1 & 12.9 & 0.34 & 3.2 & 15.5 & 0 & 0 & 903 \\
\hline $\mathrm{HB} 0702 \mathrm{cl}$ & 14.1 & 0.50 & 4.9 & 24.0 & 0 & 0 & 908 \\
\hline $\mathrm{HB} 0702 \mathrm{cl}$ & 15.7 & 0.47 & 4.3 & 22.0 & 0 & 0 & 837 \\
\hline $\mathrm{HB} 0702 \mathrm{c} 1$ & 17.8 & 0.38 & 4.2 & 17.4 & 0 & 0 & 929 \\
\hline $\mathrm{HB} 0702 \mathrm{c} 1$ & 19.9 & 0.29 & 4.0 & 17.1 & 0 & 0 & 873 \\
\hline HB0702c1 & 22.0 & 0.40 & 4.6 & 19.8 & 0 & 0 & 720 \\
\hline
\end{tabular}


Table 3.10: Porewater Data, Continued

\begin{tabular}{|c|c|c|c|c|c|c|c|}
\hline & $\begin{array}{l}\text { Mid-Depth } \\
(\mathrm{cm})\end{array}$ & $\begin{array}{c}{[\mathrm{Ag}]} \\
(\mathrm{nmol} / \mathrm{kg})\end{array}$ & $\begin{array}{c}{[\mathrm{Pb}]} \\
(\mathrm{nmol} / \mathrm{kg}) \\
\end{array}$ & $\begin{array}{c}{[\mathrm{Cu}]} \\
(\mathrm{nmol} / \mathrm{kg}) \\
\end{array}$ & $\begin{array}{c}{[\mathrm{Fe}]} \\
(\mu \mathrm{mol} / \mathrm{kg}) \\
\end{array}$ & $\begin{array}{c}{[\mathrm{Mn}]} \\
(\mu \mathrm{mol} / \mathrm{kg}) \\
\end{array}$ & $\begin{array}{c}{[\mathrm{S}]} \\
(\mu \mathrm{mol} / \mathrm{L})\end{array}$ \\
\hline $\mathrm{HB} 0702 \mathrm{c} 2$ & 0.2 & 0.18 & 1.7 & 13.0 & 28 & & 0 \\
\hline $\mathrm{HB} 0702 \mathrm{c} 2$ & 0.6 & 0.37 & 3.9 & 20.9 & 122 & & 0 \\
\hline HB0702c2 & 1.0 & 0.38 & 4.1 & 19.4 & 122 & & 0 \\
\hline $\mathrm{HB} 0702 \mathrm{c} 2$ & 1.4 & 0.37 & 4.7 & 21.5 & 144 & & 0 \\
\hline $\mathrm{HB} 0702 \mathrm{c} 2$ & 1.8 & 0.52 & 4.1 & 20.4 & 160 & & 0 \\
\hline $\mathrm{HB} 0702 \mathrm{c} 2$ & 2.2 & 0.66 & 4.9 & 27.5 & 170 & & 0 \\
\hline HB0702c2 & 2.7 & 0.48 & 5.3 & 25.8 & 157 & & 0 \\
\hline $\mathrm{HB} 0702 \mathrm{c} 2$ & 3.3 & 0.46 & 4.9 & 24.7 & 144 & & 0 \\
\hline HB0702c2 & 3.9 & 0.64 & 7.4 & 38.0 & 141 & & 0 \\
\hline $\mathrm{HB} 0702 \mathrm{c} 2$ & 4.5 & 0.92 & 8.7 & 45.3 & 161 & & 0 \\
\hline HB0702c2 & 5.1 & 0.26 & 3.3 & 18.2 & 70 & & 0 \\
\hline HB0702c2 & 5.7 & 0.27 & 3.2 & 18.0 & 45 & & 0 \\
\hline $\mathrm{HB} 0702 \mathrm{c} 2$ & 6.5 & & & & 7 & & 27 \\
\hline $\mathrm{HB} 0702 \mathrm{c} 2$ & 7.5 & 0.22 & 2.0 & 13.4 & 0 & & 252 \\
\hline $\mathrm{HB} 0702 \mathrm{c} 2$ & 8.5 & 0.20 & 1.7 & 10.4 & 0 & & 322 \\
\hline HB0702c2 & 9.4 & 0.41 & 3.1 & 14.8 & 0 & & 541 \\
\hline HB0702c2 & 10.4 & 0.31 & 2.4 & 12.6 & 0 & & 624 \\
\hline $\mathrm{HB} 0702 \mathrm{c} 2$ & 11.4 & & & & & & 708 \\
\hline HB0702c2 & 13.0 & 0.53 & 4.4 & 19.5 & 0 & & 794 \\
\hline HB0702c2 & 15.1 & 0.43 & 4.6 & 20.1 & 0 & & 869 \\
\hline HB0702c2 & 17.2 & 0.44 & 6.2 & 24.7 & 0 & & 845 \\
\hline $\mathrm{HB} 0702 \mathrm{c} 2$ & 19.3 & 0.46 & 4.6 & 23.1 & 0 & & 820 \\
\hline $\mathrm{HB} 0702 \mathrm{c} 2$ & 21.4 & 0.38 & 5.4 & 20.9 & 0 & & 681 \\
\hline $\mathrm{HB} 0702 \mathrm{c} 2$ & 23.5 & 0.37 & 4.9 & 21.4 & 0 & & 823 \\
\hline $\mathrm{HB} 0902 \mathrm{c} 1$ & 0.2 & 0.14 & 0.6 & 15.6 & 37 & & 0 \\
\hline $\mathrm{HB} 0902 \mathrm{c} 1$ & 0.5 & 0.09 & 0.8 & 8.4 & 156 & & 0 \\
\hline $\mathrm{HB} 0902 \mathrm{cl}$ & 0.9 & 0.18 & 2.3 & 14.8 & 158 & & 0 \\
\hline $\mathrm{HB} 0902 \mathrm{c} 1$ & 1.2 & 0.11 & 0.6 & 9.7 & 185 & & 0 \\
\hline $\mathrm{HB} 0902 \mathrm{c} 1$ & 1.6 & 0.15 & 1.6 & 12.3 & 97 & & 0 \\
\hline HB0902c1 & 1.9 & 0.07 & 0.9 & 11.5 & 114 & & 0 \\
\hline HB0902c1 & 2.3 & 0.23 & 2.1 & 14.3 & 173 & & 0 \\
\hline HB0902c1 & 2.8 & 0.28 & 2.8 & 19.9 & 128 & & 0 \\
\hline HB0902c1 & 3.3 & 0.39 & 4.6 & 24.8 & 56 & & 0 \\
\hline HB0902cl & 3.8 & 0.46 & 5.7 & 31.3 & 57 & & 0 \\
\hline $\mathrm{HB} 0902 \mathrm{cl}$ & 4.3 & 0.55 & 5.2 & 32.4 & 52 & & 0 \\
\hline HB0902c1 & 4.8 & 0.56 & 6.9 & 32.5 & 27 & & 0 \\
\hline HB0902c1 & 5.6 & 0.23 & 2.8 & 12.9 & 6 & & $\cdot 1$ \\
\hline HB0902c1 & 6.8 & 0.54 & 6.2 & 33.5 & 3 & & 87 \\
\hline HB0902c1 & 8.0 & 0.23 & 3.0 & 19.2 & 0 & & 304 \\
\hline HB0902c1 & 9.2 & 0.27 & 4.7 & 22.0 & 0 & & 422 \\
\hline HB0902c1 & 10.5 & 0.13 & 1.3 & 13.5 & 0 & & 455 \\
\hline HB0902c1 & 11.7 & 0.18 & 2.4 & 14.6 & 0 & & 444 \\
\hline HB0902c1 & 12.9 & 0.26 & 5.0 & 21.2 & 0 & & 441 \\
\hline HB0902c1 & 14.1 & 0.36 & 3.2 & 17.9 & 0 & & 409 \\
\hline HB0902c1 & 15.7 & 0.22 & 2.2 & 12.6 & 0 & & 387 \\
\hline HB0902c1 & 17.8 & 0.49 & 4.4 & 20.0 & 0 & & 348 \\
\hline HB0902c1 & 19.9 & 0.34 & 3.7 & 19.2 & 0 & & 375 \\
\hline HB0902c1 & 22.0 & 0.59 & 8.4 & 35.0 & 0 & & 362 \\
\hline
\end{tabular}


Table 3.10: Porewater Data, Continued

\begin{tabular}{|c|c|c|c|c|c|c|c|}
\hline & $\begin{array}{l}\text { Mid-Depth } \\
(\mathrm{cm})\end{array}$ & $\begin{array}{c}{[\mathrm{Ag}]} \\
(\mathrm{nmol} / \mathrm{kg})\end{array}$ & $\begin{array}{c}{[\mathrm{Pb}]} \\
(\mathrm{nmol} / \mathrm{kg})\end{array}$ & $\begin{array}{c}{[\mathrm{Cu}]} \\
(\mathrm{nmol} / \mathrm{kg})\end{array}$ & $\begin{array}{c}{[\mathrm{Fe}]} \\
(\mu \mathrm{mol} / \mathrm{kg})\end{array}$ & $\begin{array}{c}{[\mathrm{Mn}]} \\
(\mu \mathrm{mol} / \mathrm{kg})\end{array}$ & $\begin{array}{c}{[\mathrm{S}]} \\
(\mu \mathrm{mol} / \mathrm{L})\end{array}$ \\
\hline HB1003c1 & 0.1 & 0.17 & 1.0 & 24.9 & 10 & 12 & 0 \\
\hline HB1003c1 & 0.4 & 0.40 & 2.1 & 25.1 & 78 & 16 & 0 \\
\hline $\mathrm{HB} 1003 \mathrm{c1}$ & 0.6 & 0.19 & 1.3 & 13.2 & 177 & 17 & 0 \\
\hline HB1003c1 & 0.9 & 0.20 & 1.9 & 16.8 & 184 & 17 & 0 \\
\hline HB1003c1 & 1.1 & 0.22 & 2.4 & 14.0 & 137 & 16 & 0 \\
\hline HB1003c1 & 1.4 & 0.26 & 5.9 & 20.0 & 126 & 13 & 0 \\
\hline HB1003c1 & 1.8 & 0.19 & 2.3 & 13.6 & 106 & 13 & 0 \\
\hline HB1003c1 & 2.3 & 0.16 & 2.2 & 12.6 & 57 & 9 & 0 \\
\hline HB1003c1 & 2.8 & 0.17 & 2.4 & 12.8 & 36 & 4 & 0 \\
\hline HB1003c1 & 3.4 & 0.15 & 2.2 & 14.3 & 32 & 7 & 0 \\
\hline HB1003c1 & 3.9 & 0.25 & 3.4 & 19.4 & 23 & 6 & 0 \\
\hline HB1003c1 & 4.4 & 0.24 & 3.2 & 19.1 & 14 & 4 & 0 \\
\hline HB1003c1 & 5.2 & 0.18 & 2.5 & 17.3 & 8 & 3 & 0 \\
\hline HB1003c1 & 6.1 & 0.35 & 4.8 & 30.3 & 5 & 3 & 90 \\
\hline HB1003c1 & 7.1 & 0.14 & 2.5 & 19.1 & 0 & 2 & 189 \\
\hline HB1003c1 & 8.0 & 0.47 & 6.1 & 36.9 & 0 & 2 & 358 \\
\hline HB1003c1 & 8.9 & 0.20 & 2.2 & 16.5 & 0 & 0 & 410 \\
\hline HB1003c1 & 9.9 & 0.19 & 1.9 & 14.0 & 0 & 0 & 440 \\
\hline $\mathrm{HB} 1003 \mathrm{cl}$ & 11.4 & 0.32 & 3.1 & 20.1 & 0 & 0 & 427 \\
\hline HB1003c1 & 13.4 & 0.47 & 4.4 & 22.0 & 0 & 0 & 428 \\
\hline $\mathrm{HB} 1003 \mathrm{c} 1$ & 15.4 & 0.37 & 4.5 & 20.3 & 0 & 0 & 368 \\
\hline $\mathrm{HB} 1003 \mathrm{cl}$ & 17.4 & 0.31 & 3.7 & 17.6 & 0 & 0 & 317 \\
\hline $\mathrm{HB} 1003 \mathrm{cl}$ & 19.4 & 0.20 & 3.1 & 16.2 & 0 & 0 & 309 \\
\hline HB1003c1 & 23.4 & 0.19 & 3.2 & 17.4 & 0 & 0 & 275 \\
\hline HB0604c1 & 0.2 & 0.05 & 0.3 & 4.0 & 21 & 5 & 0 \\
\hline HB0604c1 & 0.5 & 0.06 & 0.3 & 7.6 & 36 & 5 & 0 \\
\hline $\mathrm{HB} 0604 \mathrm{cl}$ & 0.8 & 0.11 & 0.4 & 12.8 & 44 & 5 & 0 \\
\hline $\mathrm{HB} 0604 \mathrm{c} 1$ & 1.1 & 0.25 & 0.7 & 5.9 & 74 & 4 & 0 \\
\hline HB0604c1 & 1.4 & 0.20 & 0.7 & 9.8 & 56 & 4 & 0 \\
\hline HB0604cl & 1.7 & 0.15 & 0.7 & 12.2 & 45 & 5 & 0 \\
\hline HB0604c1 & 2.0 & 0.15 & 1.1 & 18.3 & 66 & 5 & 1 \\
\hline HB0604c1 & 2.5 & 0.18 & 2.0 & 19.1 & 60 & 6 & 0 \\
\hline HB0604c1 & 2.9 & 0.27 & 2.4 & 29.5 & 41 & 7 & 1. \\
\hline HB0604c1 & 3.4 & 0.18 & 1.4 & 15.1 & 25 & 6 & 1 \\
\hline HB0604c1 & 3.8 & 0.07 & 0.7 & 6.2 & 10 & 5 & 0 \\
\hline HB0604c1 & 4.2 & 0.07 & 0.7 & 6.9 & 7 & 5 & 0 \\
\hline $\mathrm{HB} 0604 \mathrm{cl}$ & 4.7 & 0.20 & 0.8 & 7.9 & 5 & 4 & 0 \\
\hline $\mathrm{HB} 0604 \mathrm{c} 1$ & 5.1 & 0.14 & 0.3 & 6.9 & 6 & 3 & \\
\hline HBO604c1 & 5.6 & 0.10 & 0.9 & 10.2 & 4 & 4 & 5 \\
\hline $\mathrm{HB} 0604 \mathrm{c} 1$ & 6.0 & 0.10 & 0.8 & 8.1 & 0 & 2 & 23 \\
\hline HB0604c1 & 6.5 & 0.21 & 0.9 & 12.9 & 0 & 3 & 0 \\
\hline $\mathrm{HB} 0604 \mathrm{cl}$ & 6.9 & 0.20 & 1.4 & 10.2 & 0 & 2 & 43 \\
\hline HB0604c1 & 8.2 & 0.11 & 0.5 & 6.9 & 0 & 2 & 73 \\
\hline HB0604c1 & 10.3 & 0.23 & 0.6 & 7.7 & 0 & 0 & 121 \\
\hline $\mathrm{HB} 0604 \mathrm{cl}$ & 12.4 & 0.13 & 1.0 & 7.8 & 0 & 0 & 540 \\
\hline HB0604cI & 14.5 & 0.13 & 0.8 & 7.4 & 0 & 0 & 634 \\
\hline HB0604c1 & 16.7 & 0.15 & 0.8 & 5.0 & 0 & 0 & 788 \\
\hline HB0604c1 & 18.8 & 0.09 & 1.1 & 4.9 & 0 & 0 & 890 \\
\hline
\end{tabular}


Table 3.10: Porewater Data, Continued

\begin{tabular}{|c|c|c|c|c|c|c|c|}
\hline & $\begin{array}{l}\text { Mid-Depth } \\
(\mathrm{cm})\end{array}$ & $\begin{array}{c}{[\mathrm{Ag}]} \\
(\mathrm{nmol} / \mathrm{kg})\end{array}$ & $\begin{array}{c}{[\mathrm{Pb}]} \\
(\mathrm{nmol} / \mathrm{kg})\end{array}$ & $\begin{array}{c}{[\mathrm{Cu}]} \\
(\mathrm{nmol} / \mathrm{kg}) \\
\end{array}$ & $\begin{array}{c}{[\mathrm{Fe}]} \\
(\mu \mathrm{mol} / \mathrm{kg}) \\
\end{array}$ & $\begin{array}{c}{[\mathrm{Mn}]} \\
(\mu \mathrm{mol} / \mathrm{kg})\end{array}$ & $\begin{array}{c}{[\mathrm{S}]} \\
(\mu \mathrm{mol} / \mathrm{L})\end{array}$ \\
\hline HB0604c2 & 0.2 & 0.03 & 0.6 & 12.7 & 37 & 12 & 0 \\
\hline $\mathrm{HB} 0604 \mathrm{c} 2$ & 0.5 & 0.55 & 0.5 & 25.9 & 72 & 13 & 0 \\
\hline HB0604c2 & 0.8 & 0.08 & 0.6 & 14.1 & 109 & 12 & 0 \\
\hline $\mathrm{HB} 0604 \mathrm{c} 2$ & 1.1 & 0.09 & 0.6 & 20.1 & 110 & 12 & 0 \\
\hline HB0604c2 & $1: 4$ & 0.07 & 0.7 & 10.0 & 150 & 11 & 0 \\
\hline $\mathrm{HB} 0604 \mathrm{c} 2$ & 1.7 & 0.16 & 1.5 & 28.1 & 171 & 11 & 0 \\
\hline HB0604c2 & 2.1 & 0.34 & 2.7 & 37.7 & 233 & 14 & 0 \\
\hline HB0604c2 & 2.6 & 0.27 & 2.1 & 30.1 & 268 & 20 & 1 \\
\hline HB0604c2 & 3.1 & 0.41 & 3.8 & 37.3 & 226 & 22 & 1 \\
\hline HB0604c2 & 3.7 & 0.09 & 0.9 & 11.7 & 90 & 23 & 1 \\
\hline HB0604c2 & 4.2 & 0.08 & 1.0 & 8.5 & 63 & 23 & 1 \\
\hline $\mathrm{HB} 0604 \mathrm{c} 2$ & 4.7 & 0.14 & 1.4 & 13.9 & 51 & 22 & 1 \\
\hline HB0604c2 & 5.3 & 0.12 & 1.2 & 10.4 & 16 & 20 & 1 \\
\hline HB0604c2 & 5.8 & 0.13 & 1.4 & 14.2 & 10 & 16 & 1 \\
\hline HB0604c2 & 6.3 & 0.11 & 1.3 & 11.1 & 6 & 13 & 2 \\
\hline HB0604c2 & 6.9 & 0.11 & 1.1 & 10.8 & 6 & 16 & 4 \\
\hline HB0604c2 & 7.4 & 0.13 & 1.4 & 11.3 & & 14 & 0 \\
\hline HB0604c2 & 7.9 & 0.22 & 2.7 & 20.6 & 0 & 13 & 25 \\
\hline HB0604c2 & 9.2 & 0.18 & 1.8 & 20.0 & 0 & 11 & 72 \\
\hline HB0604c2 & 11.3 & 0.13 & 0.6 & 7.7 & 0 & 2 & 161 \\
\hline HB0604c2 & 13.3 & 0.16 & 1.4 & 9.7 & 0 & 0 & 572 \\
\hline HB0604c2 & 18.0 & 0.15 & 0.8 & 9.8 & 0 & 0 & 636 \\
\hline HB0604c2 & 20.0 & 0.34 & 2.6 & 15.3 & 0 & 0 & 809 \\
\hline HIB0604c2 & 26.2 & 0.32 & 3.6 & 18.1 & 0 & 0 & 842 \\
\hline MB0202c1 & 0.1 & 0.12 & 1.0 & 22.3 & 0 & & 0 \\
\hline MB0202c1 & 0.4 & 0.06 & 1.1 & 16.6 & 6 & & 0 \\
\hline MB0202c1 & 0.6 & 0.09 & 1.4 & 12.8 & 30 & & 0 \\
\hline MB0202c1 & 0.9 & 0.10 & 2.7 & 12.6 & 85 & & 0 \\
\hline $\mathrm{MB} 0202 \mathrm{cl}$ & 1.1 & 0.13 & 3.7 & 14.8 & 101 & & 0 \\
\hline MB0202c1 & 1.4 & 0.12 & 3.3 & 21.7 & 99 & & 0 \\
\hline $\mathrm{MB} 0202 \mathrm{c} 1$ & 1.7 & 0.14 & 4.5 & 16.7 & 117 & & 0 \\
\hline MB0202c1 & 2.2 & 0.16 & 4.6 & 23.7 & 101 & & 0 \\
\hline MB0202c1 & 2.7 & 0.08 & 1.9 & 11.0 & 77 & & 0 \\
\hline $\mathrm{MB} 0202 \mathrm{cl}$ & 3.1 & 0.11 & 2.1 & 13.7 & 66 & & 0 \\
\hline MB0202c1 & 3.6 & 0.14 & 2.0 & 17.1 & 51 & & 0 \\
\hline MB0202c1 & 4.1 & 0.10 & 2.3 & 19.8 & 53 & & 0 \\
\hline MB0202c1 & 4.9 & 0.13 & 1.5 & 13.0 & 70 & & 0 \\
\hline MB0202c1 & 6.2 & 0.05 & 2.0 & 10.5 & 102 & & 0 \\
\hline MB0202cl & 7.4 & 0.13 & 5.9 & 17.7 & 135 & & 0 \\
\hline $\mathrm{MB} 0202 \mathrm{c} 1$ & 8.6 & 0.09 & 4.2 & 17.0 & 127 & & 0 \\
\hline MB0202c1 & 9.9 & 0.11 & 5.2 & 23.2 & 113 & & 0 \\
\hline MB0202c1 & 11.1 & 0.15 & 6.8 & 22.1 & 113 & & 0 \\
\hline $\mathrm{MB} 0202 \mathrm{cl}$ & 12.8 & 0.18 & 7.7 & 26.0 & 95 & & 0 \\
\hline $\mathrm{MB} 0202 \mathrm{c} 1$ & 15.0 & 0.11 & 4.5 & 26.1 & 65 & & 0 \\
\hline $\mathrm{MB} 0202 \mathrm{c} 1$ & 17.1 & 0.21 & 7.3 & 22.9 & 91 & & 0 \\
\hline $\mathrm{MB0202 \textrm {c } 1}$ & 19.3 & 0.14 & 6.2 & 16.8 & 59 & & 0 \\
\hline $\mathrm{MBO} 202 \mathrm{c} 1$ & 21.5 & 0.12 & 6.3 & 17.8 & 80 & & 0 \\
\hline $\mathrm{MB} 0202 \mathrm{c} 1$ & 23.6 & 0.11 & 6.8 & 17.0 & 51 & & 0 \\
\hline
\end{tabular}


Table 3.10: Porewater Data, Continued

\begin{tabular}{|c|c|c|c|c|c|c|c|}
\hline & $\begin{array}{l}\text { Mid-Depth } \\
(\mathrm{cm})\end{array}$ & $\begin{array}{c}{[\overline{\mathrm{Ag}}]} \\
(\mathrm{nmol} / \mathrm{kg})\end{array}$ & $\begin{array}{c}{[\mathrm{Pb}]} \\
(\mathrm{nmol} / \mathrm{kg})\end{array}$ & $\begin{array}{c}{[\mathrm{Cu}]} \\
(\mathrm{nmol} / \mathrm{kg})\end{array}$ & $\begin{array}{c}{[\mathrm{Fe}]} \\
(\mu \mathrm{mol} / \mathrm{kg})\end{array}$ & $\begin{array}{c}{[\mathrm{Mn}]} \\
(\mu \mathrm{mol} / \mathrm{kg})\end{array}$ & $\begin{array}{c}{[\mathrm{S}]} \\
(\mu \mathrm{mol} / \mathrm{L})\end{array}$ \\
\hline $\mathrm{MB0502 \textrm {c } 1}$ & 0.2 & 0.26 & 1.3 & 43.8 & 0 & & 0 \\
\hline MB0502c1 & 0.5 & 0.14 & 1.0 & 28.0 & 0 & & 0 \\
\hline MB0502c1 & 0.9 & 0.07 & 1.2 & 16.7 & 19 & & 0 \\
\hline $\mathrm{MB} 0502 \mathrm{cl}$ & 1.2 & 0.07 & 2.2 & 13.9 & 35 & & 0 \\
\hline MB0502c1 & 1.6 & 0.08 & 3.2 & 15.2 & 33 & & 0 \\
\hline $\mathrm{MB0502 \textrm {cl }}$ & 1.9 & 0.16 & 4.6 & 20.6 & 85 & & 0 \\
\hline $\mathrm{MB} 0502 \mathrm{c} 1$ & 2.3 & 0.20 & 4.1 & 19.5 & 57 & & 0 \\
\hline MB0502c1 & 2.7 & 0.29 & 7.0 & 23.0 & 89 & & 0 \\
\hline $\mathrm{MB} 0502 \mathrm{c} 1$ & 3.3 & 0.10 & 4.0 & 22.2 & 81 & & 0 \\
\hline $\mathrm{MB} 0502 \mathrm{cl}$ & 3.8 & 0.10 & 3.2 & 16.0 & 78 & & 0 \\
\hline $\mathrm{MB} 0502 \mathrm{cl}$ & 4.4 & 0.17 & 3.0 & 31.3 & 83 & & 0 \\
\hline MB0502cl & 4.9 & 0.05 & 1.8 & 12.7 & 89 & & 0 \\
\hline $\mathrm{MB} 0502 \mathrm{cl}$ & 5.7 & 0.60 & 1.3 & 14.6 & 40 & & 0 \\
\hline MB0502c1 & 6.8 & 0.07 & 1.2 & 12.9 & 97 & & 0 \\
\hline $\mathrm{MB} 0502 \mathrm{cl}$ & 7.8 & 0.06 & 2.7 & 14.0 & 142 & & 0 \\
\hline MB0502cl & 8.9 & 0.05 & 2.0 & 7.9 & 36 & & 0 \\
\hline MB0502c1 & 9.9 & 0.05 & 1.5 & 12.3 & 4 & & 0 \\
\hline MB0502c1 & 11.0 & 0.06 & 2.3 & 11.8 & 78 & & 0 \\
\hline MB0502c1 & 12.5 & 0.02 & 1.4 & 7.9 & 71 & & 0 \\
\hline $\mathrm{MB} 0502 \mathrm{c} 1$ & 16.3 & 0.06 & 2.6 & 11.4 & 64 & & 0 \\
\hline $\mathrm{MB} 0502 \mathrm{c} 1$ & 20.1 & 0.03 & 1.9 & 8.7 & 62 & & 0 \\
\hline MB0502c1 & 23.9 & 0.04 & 4.2 & 11.3 & 55 & & 0 \\
\hline MB0502c1 & 27.7 & 0.04 & 3.1 & 10.2 & 43 & & 0 \\
\hline $\mathrm{MB} 0502 \mathrm{c} 1$ & 31.5 & 0.01 & 1.7 & 13.3 & 24 & & 0 \\
\hline $\mathrm{MB} 1002 \mathrm{c} 1$ & 0.2 & 0.08 & 0.7 & 17.4 & 0 & & 0 \\
\hline MB1002c1 & 0.5 & 0.07 & 0.6 & 12.6 & 36 & & 0 \\
\hline $\mathrm{MB} 1002 \mathrm{c} 1$ & 0.8 & 0.09 & 1.2 & 13.8 & 73 & & 0 \\
\hline $\mathrm{MB} 1002 \mathrm{c} 1$ & 1.2 & 0.17 & 5.4 & 18.7 & 114 & & 0 \\
\hline $\mathrm{MB1002 \textrm {c } 1}$ & 1.5 & 0.07 & 2.5 & 9.4 & 60 & & 0 \\
\hline MB1002c1 & 1.8 & 0.07 & 1.8 & 9.0 & 71 & & 0 \\
\hline $\mathrm{MB} 1002 \mathrm{c} 1$ & 2.2 & 0.15 & 5.5 & 18.8 & 57 & & 0 \\
\hline MB1002c1 & 2.7 & 0.21 & 8.6 & 21.8 & 55 & & 0 \\
\hline $\mathrm{MB1002 \textrm {c } 1}$ & 3.2 & 0.09 & 3.4 & 16.6 & 57 & & 0 \\
\hline $\mathrm{MB1002 \textrm {c } 1}$ & 3.6 & 0.07 & 2.4 & 12.8 & 46 & & 0 \\
\hline $\mathrm{MB} 1002 \mathrm{c} 1$ & 4.1 & 0.07 & 1.4 & 9.6 & 48 & & 0 \\
\hline $\mathrm{MB} 1002 \mathrm{cl}$ & 4.6 & 0.12 & 2.8 & 31.1 & 40 & & 0 \\
\hline MB1002c1 & 5.4 & 0.04 & 2.0 & 6.6 & 35 & & 0 \\
\hline $\mathrm{MB} 1002 \mathrm{cl}$ & 6.7 & 0.03 & 1.4 & 6.5 & 37 & & 0 \\
\hline $\mathrm{MB} 1002 \mathrm{c} 1$ & 7.9 & 0.05 & 1.3 & 6.1 & 35 & & 0 \\
\hline $\mathrm{MB} 1002 \mathrm{c} 1$ & 9.2 & 0.03 & 1.2 & 4.6 & 45 & & 0 \\
\hline $\mathrm{MB} 1002 \mathrm{c} 1$ & 10.4 & 0.02 & 1.6 & 3.8 & 51 & & 0 \\
\hline $\mathrm{MB} 1002 \mathrm{c} 1$ & 11.7 & 0.02 & 1.4 & 6.6 & 71 & & 0 \\
\hline $\mathrm{MB} 1002 \mathrm{cl}$ & 13.3 & 0.03 & 2.1 & & 50 & & 0 \\
\hline $\mathrm{MB1002c1}$ & 17.3 & 0.04 & 2.1 & 4.2 & 40 & & 0 \\
\hline $\mathrm{MB1002 \textrm {c } 1}$ & 21.3 & 0.04 & 1.7 & 6.2 & 40 & & 0 \\
\hline $\mathrm{MB1002c1}$ & 25.3 & 0.02 & 1.1 & 5.0 & 64 & & 0 \\
\hline MB1002c1 & 29.3 & 0.02 & 0.7 & 4.0 & 81 & & 0 \\
\hline MB1002c1 & 35.3 & 0.04 & 2.3 & 7.0 & 178 & & 0 \\
\hline
\end{tabular}




\subsubsection{Additional Figures}



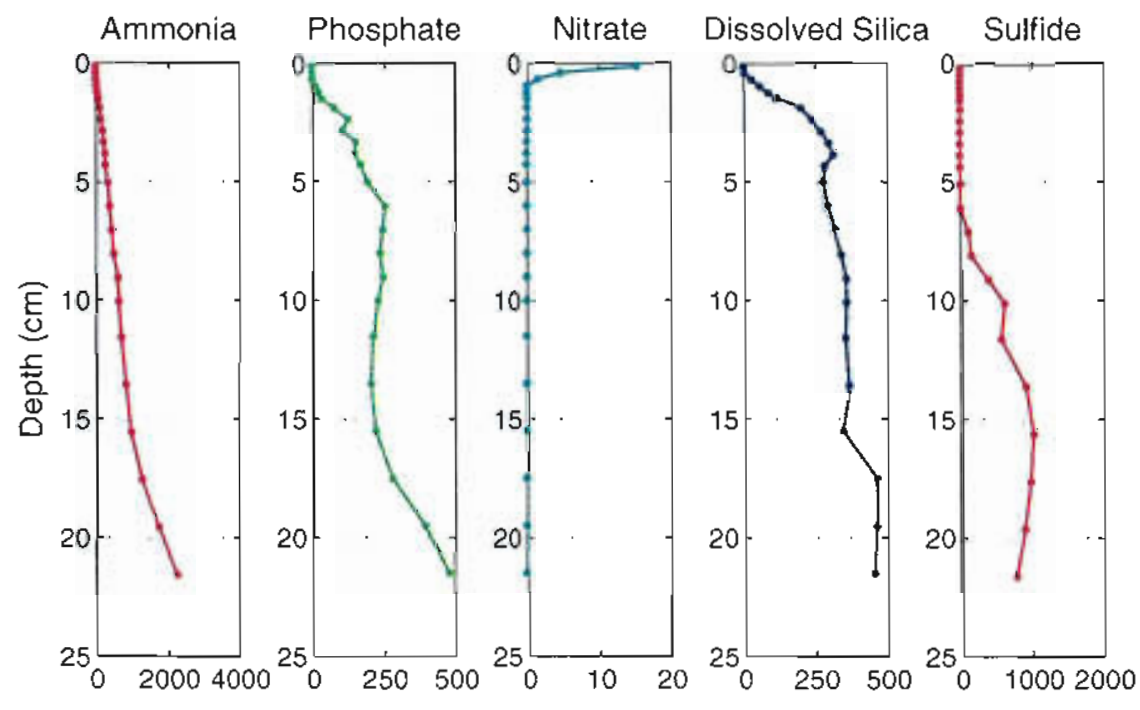

Concentration $(\mu \mathrm{mol} / \mathrm{L})$
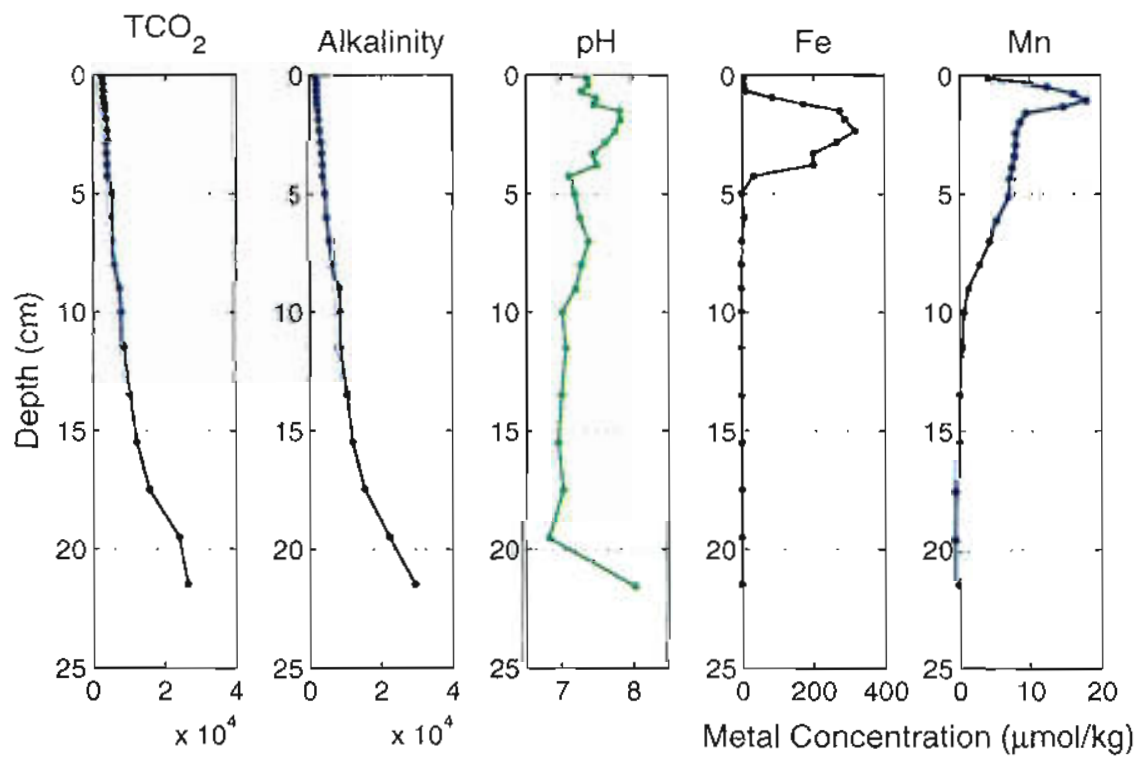

Concentration $(\mu \mathrm{mol} / \mathrm{L})$

Figure 3-30: Porewater profiles of nutrients, sulfate, sulfide, total carbon dioxide, alkalinity, calculated $\mathrm{pH}$,iron and manganese for Hingham Bay January 2002 (core 2). 

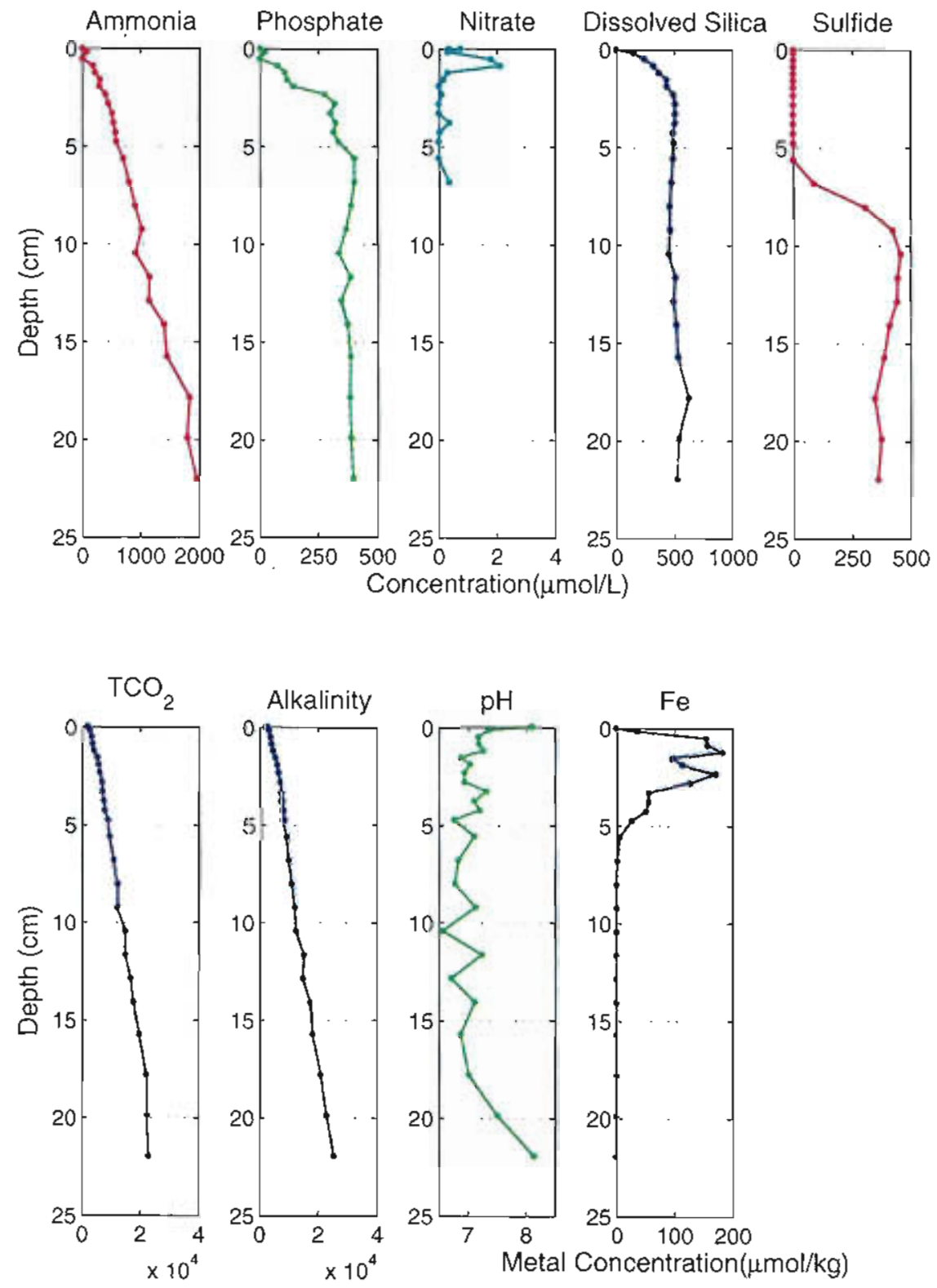

Concentration $(\mu \mathrm{mol} / \mathrm{L})$

Figure 3-31: Porewater profiles of nutrients, sulfate, sulfide, total carbon dioxide, alkalinity, calculated pH, iron and manganese for Hingham Bay September 2002 (core 1). 

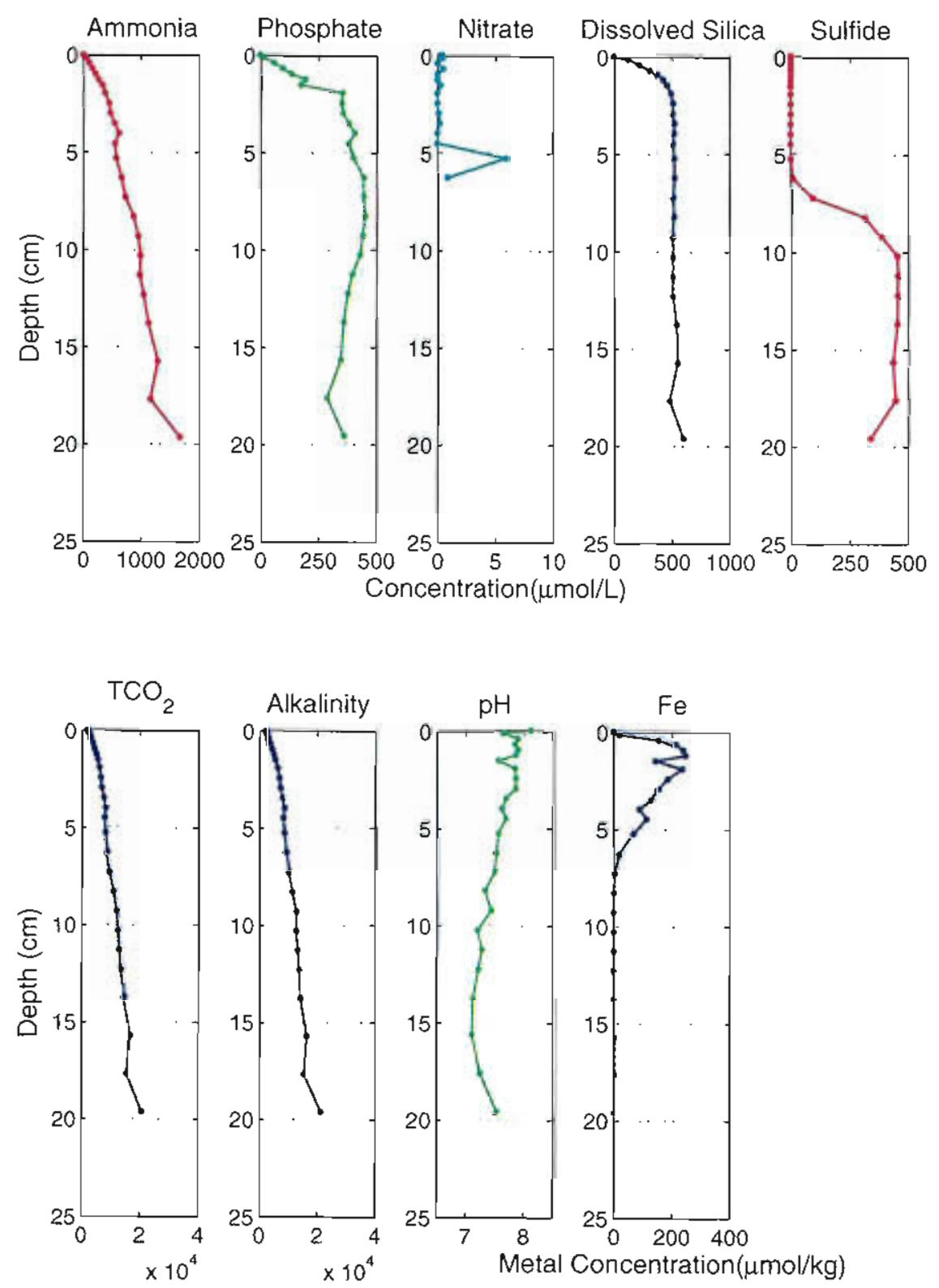

Concentration $(\mu \mathrm{mol} / \mathrm{L})$

Figure 3-32: Porewater profiles of nutrients, sulfate, sulfide, total carbon dioxide, alkalinity, calculated $\mathrm{pH}$, iron and manganese for Hingham Bay September 2002 (core 2). 

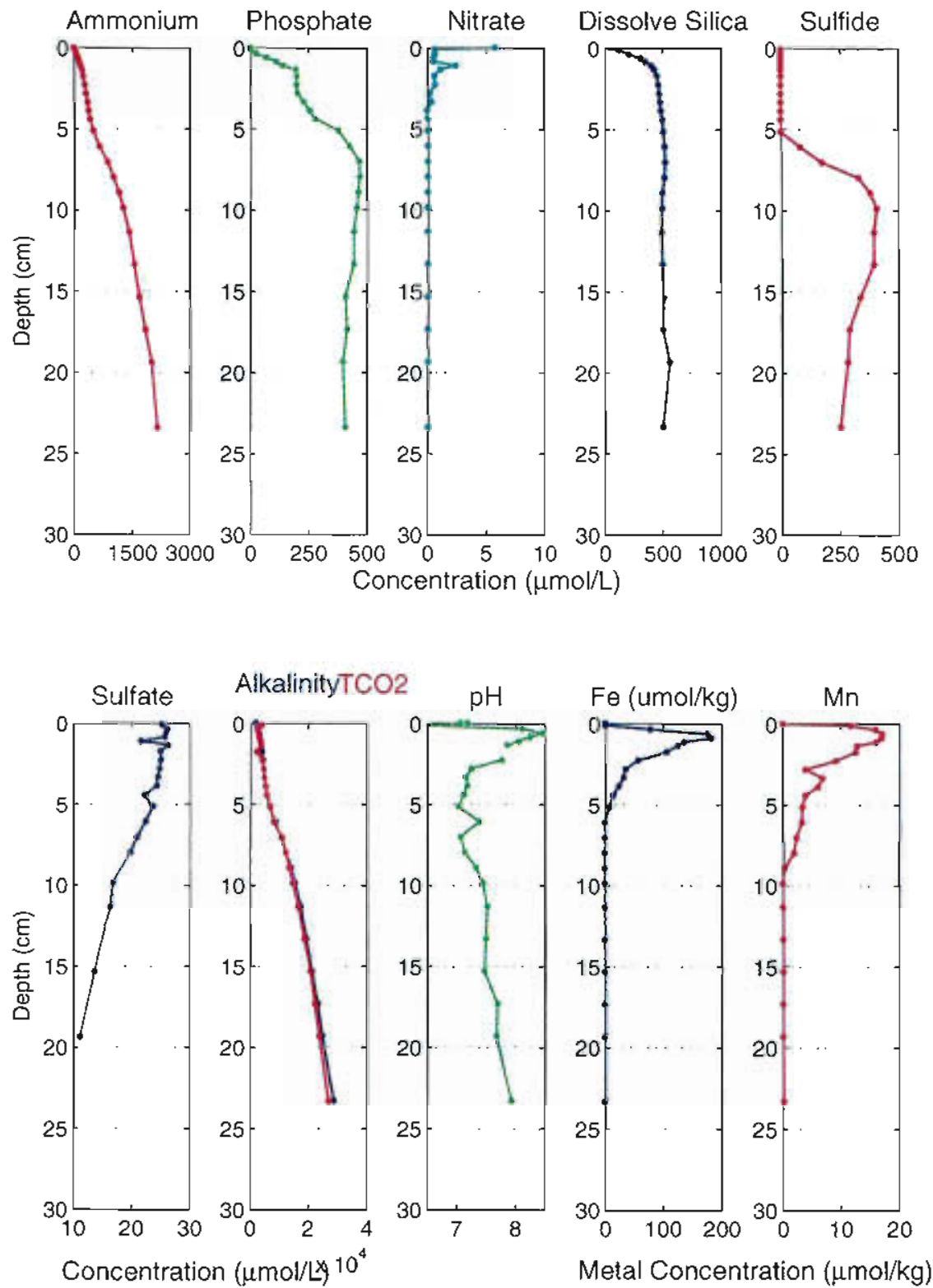

Figure 3-33: Porewater profiles of nutrients, sulfate, sulfide, total carbon dioxide, alkalinity, calculated pH,iron and manganese for Hingham Bay October 2003 (core 1). 

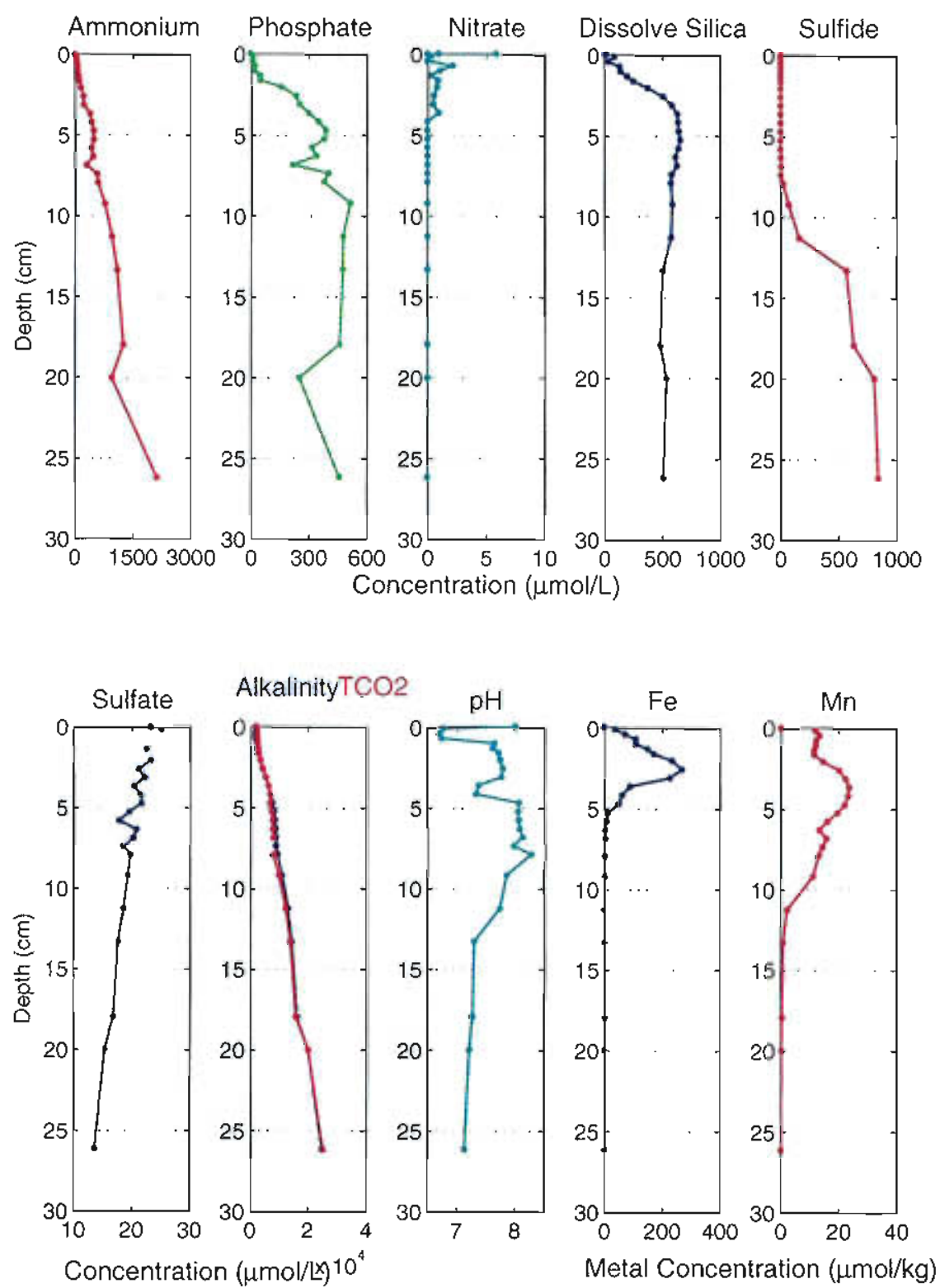

Figure 3-34: Porewater profiles of nutrients, sulfate, sulfide, iron and manganese for Hingham Bay June 2004 (core 2). 

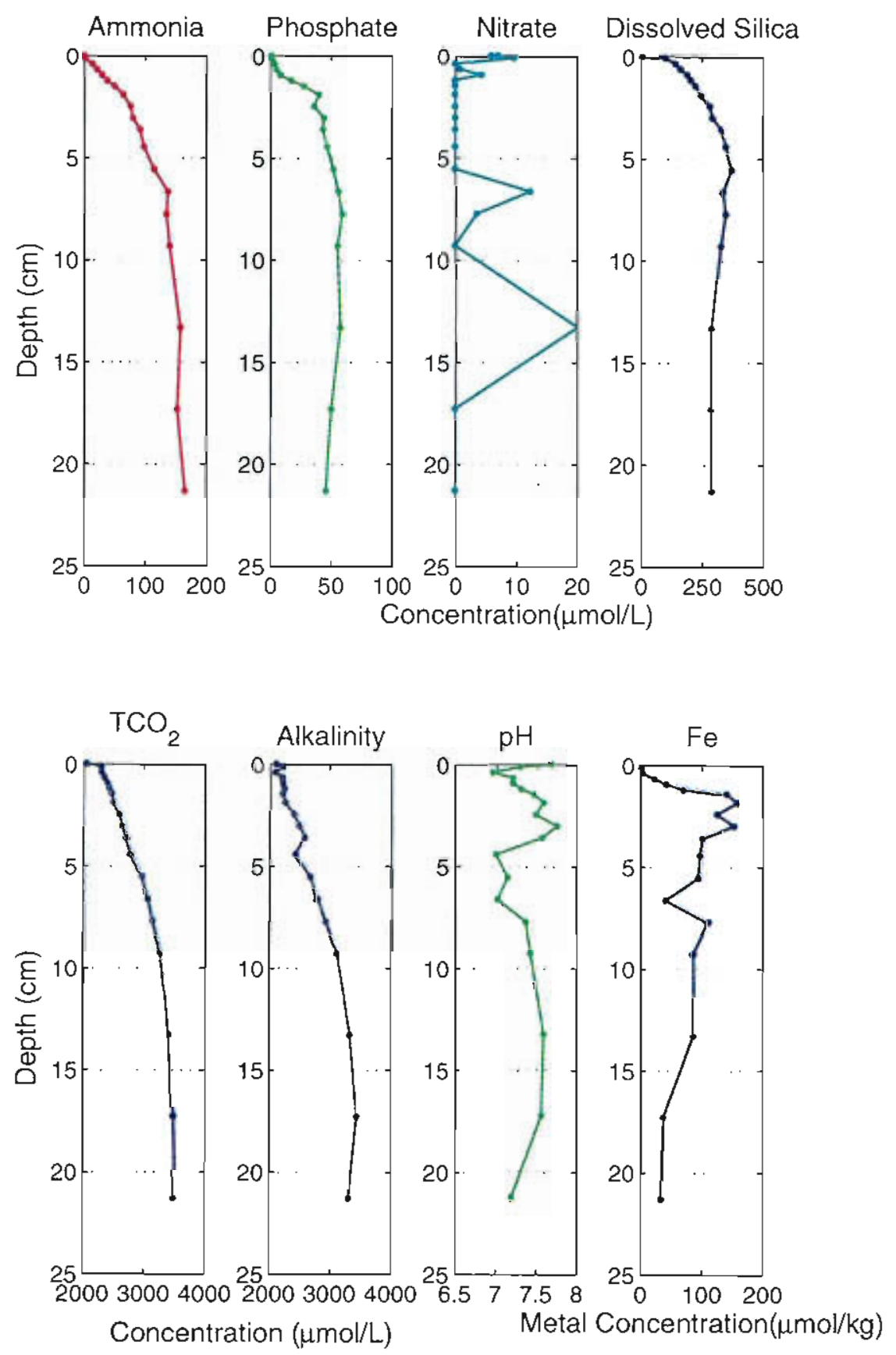

Figure 3-35: Porewater profiles of nutrients, total carbon dioxide, alkalinity, calculated pH and iron for Massachusetts Bay February 2002 (core 2). 

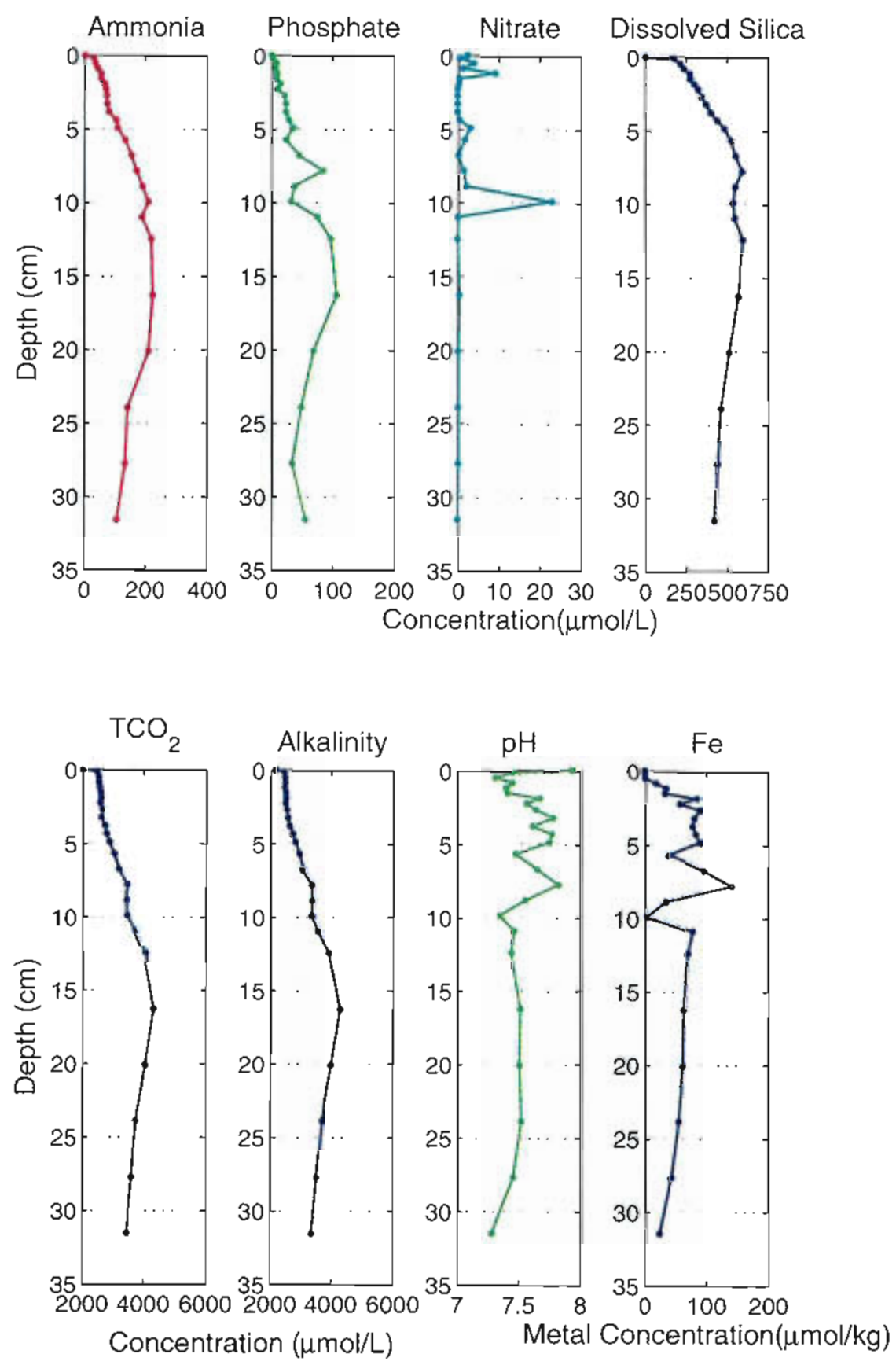

Figure 3-36: Porewater profiles of nutrients, total carbon dioxide, alkalinity, calculated $\mathrm{pH}$ and iron for Massachusetts Bay May 2002 (core 1). 

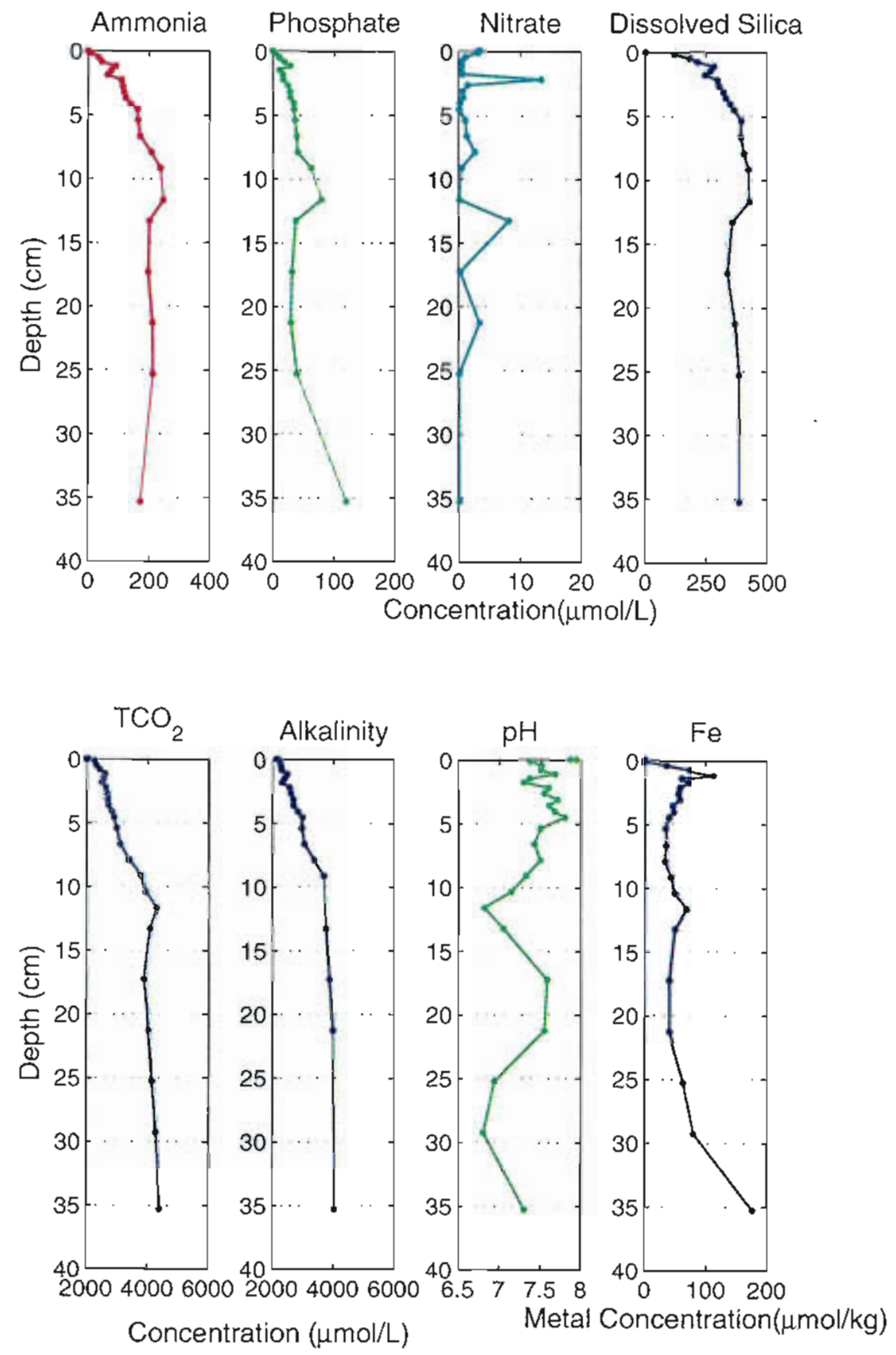

Figure 3-37: Porewater profiles of nutrients, total carbon dioxide, alkalinity, calculated pH and iron for Massachusetts Bay October 2002 (core 1). 

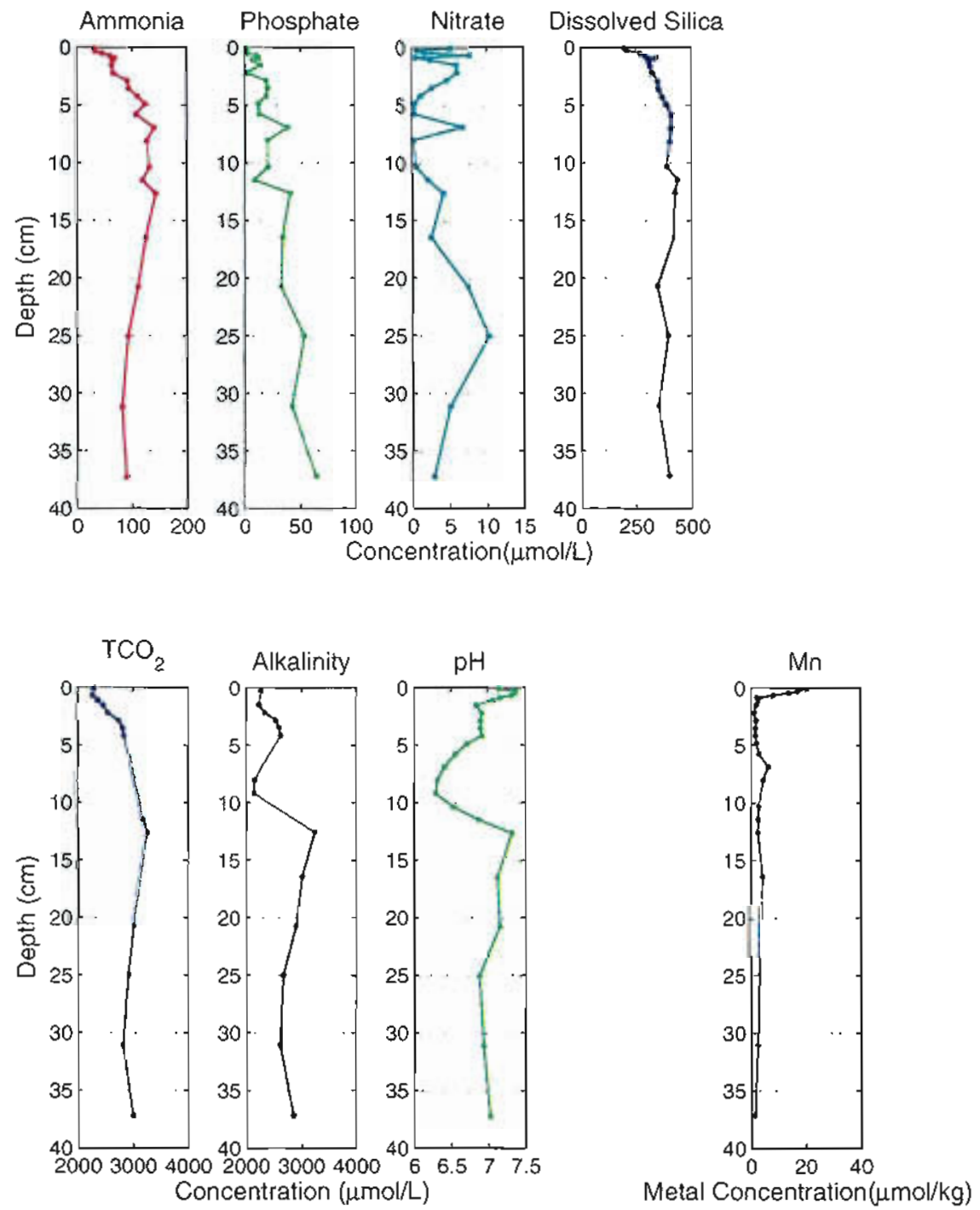

Figure 3-38: Porewater profiles of nutrients, total carbon dioxide, alkalinity, calculated pH and manganese for Massachusetts Bay September 2003 (core 2). Note that as there is no iron data the $\mathrm{pH}$ has not been corrected for iron precipitation. 

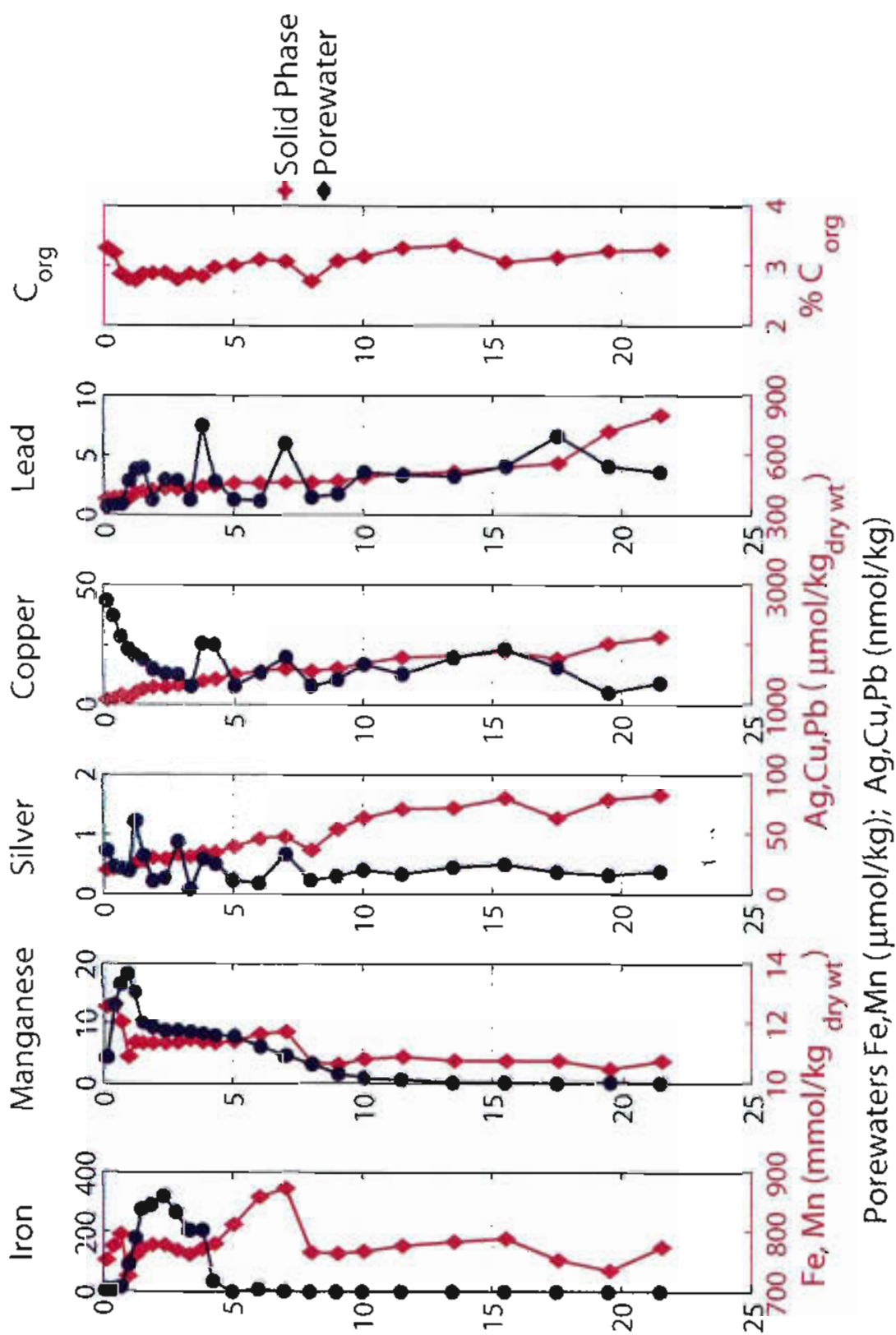

(w) $47 d \partial 0$

Figure 3-39: Solid phase and porewater profiles of silver, lead, copper, iron and manganese for Hingham Bay February 2002 (Core 2). Solid phase \% organic carbon is also shown. 

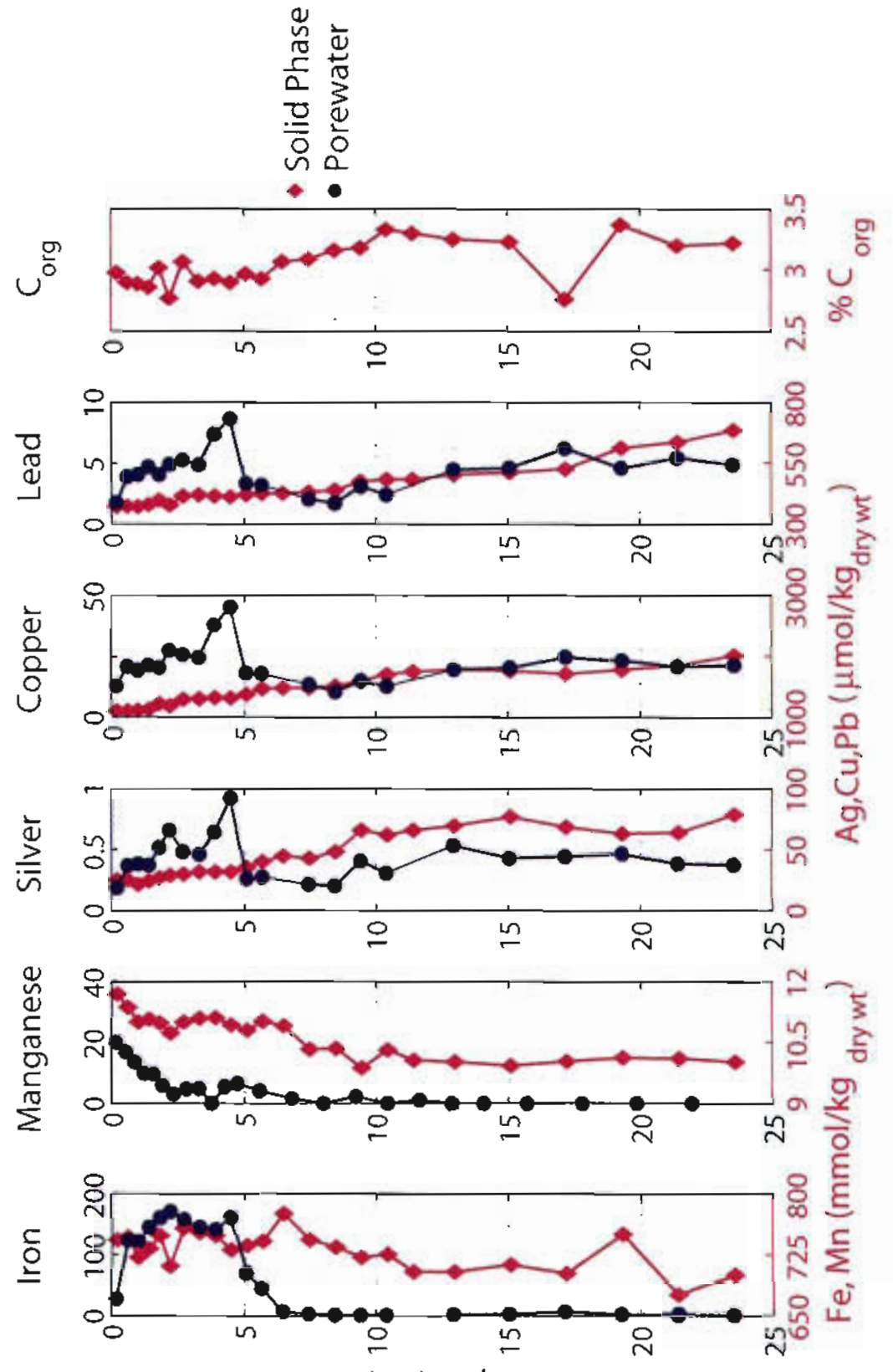

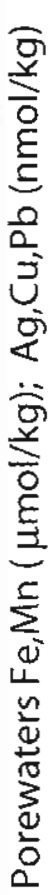

(us) $41 \mathrm{~d}=0$

Figure 3-40: Solid phase and porewater profiles of silver, lead, copper, iron and manganese for Hingham Bay July 2002 (Core 2). Solid phase \% organic carbon is also shown. Note the $\mathrm{Mn}$ porewater data is from Core 1. 

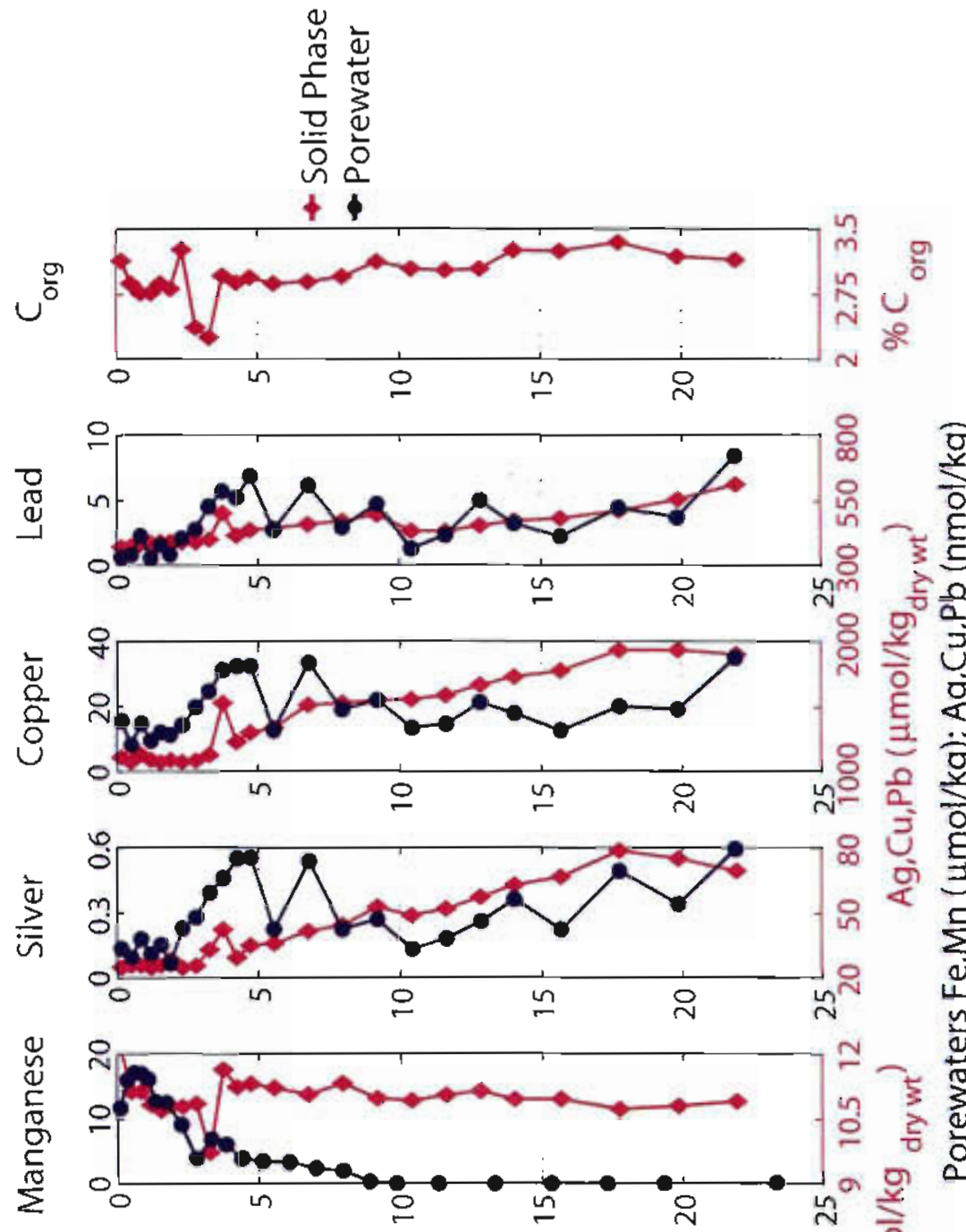

$\frac{5}{4} \frac{5}{0}$

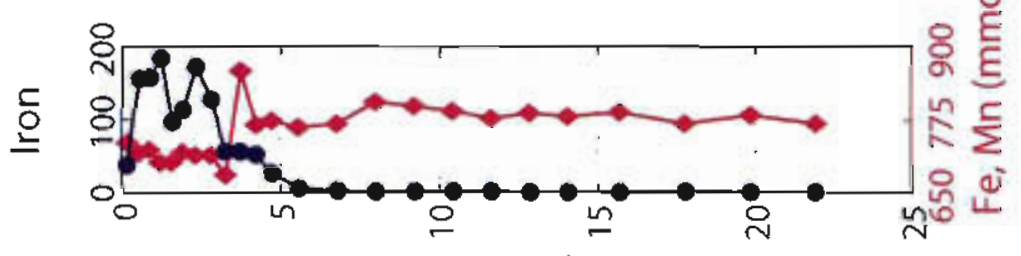

(uכ) yldəa

Figure 3-41: Solid phase and porewater profiles of silver, lead, copper, iron and manganese for Hingham Bay September 2002 (Core 1). Solid phase \% organic carbon is also shown. Note the Mn porewater data is from October 2003 Core 1. 

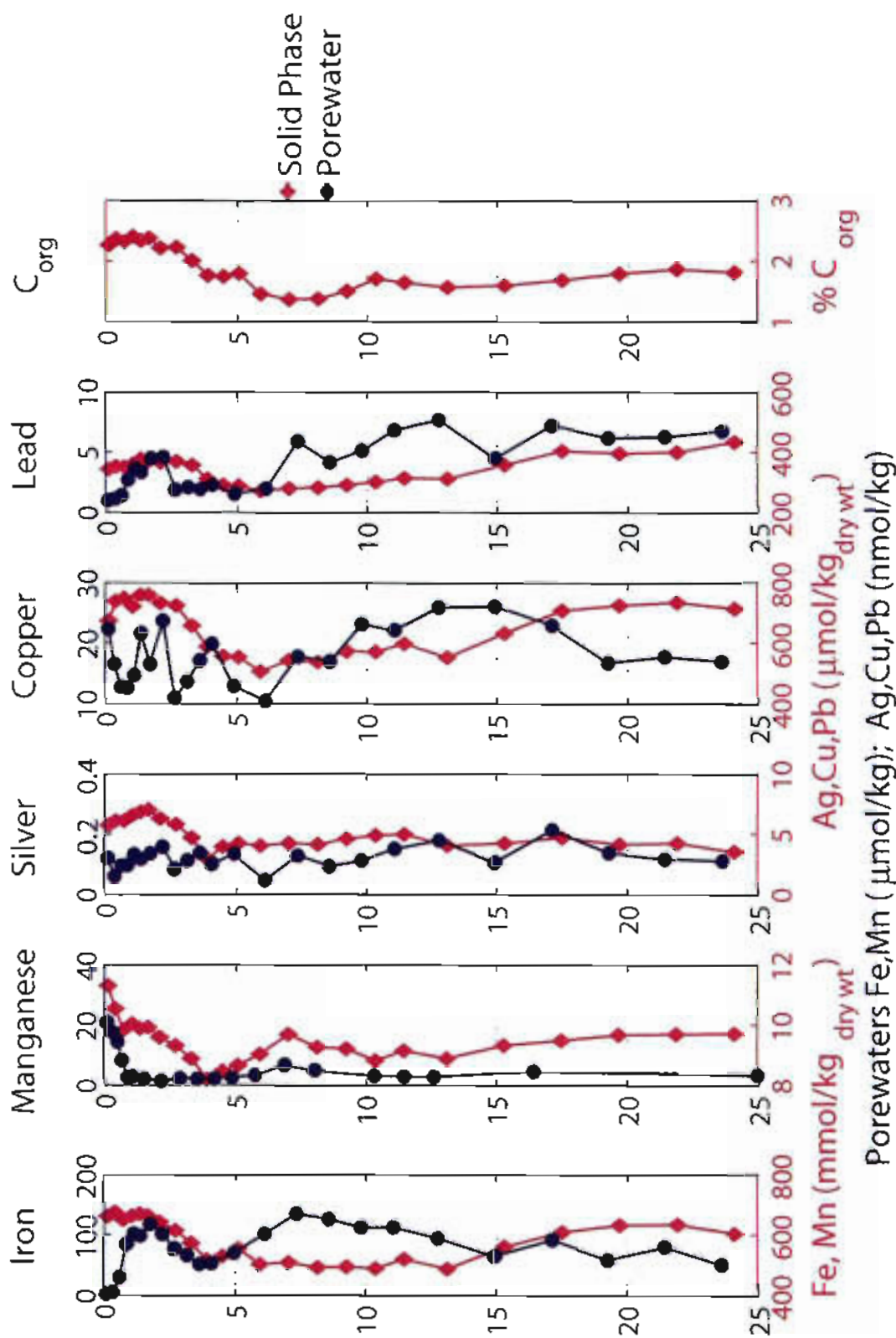

(w) yldag

Figure 3-42: Solid phase and porewater profiles of silver, lead, copper, iron, manganese and $\%$ organic carbon for Massachusetts Bay February 2002. Solid phase \% organic carbon is also shown. Note that the Mn porewater data is from September 2002. Solid phase data is from Core 3 and porewater data is from Core 1. Solid phase data courtesy M.Bothner, USGS. 

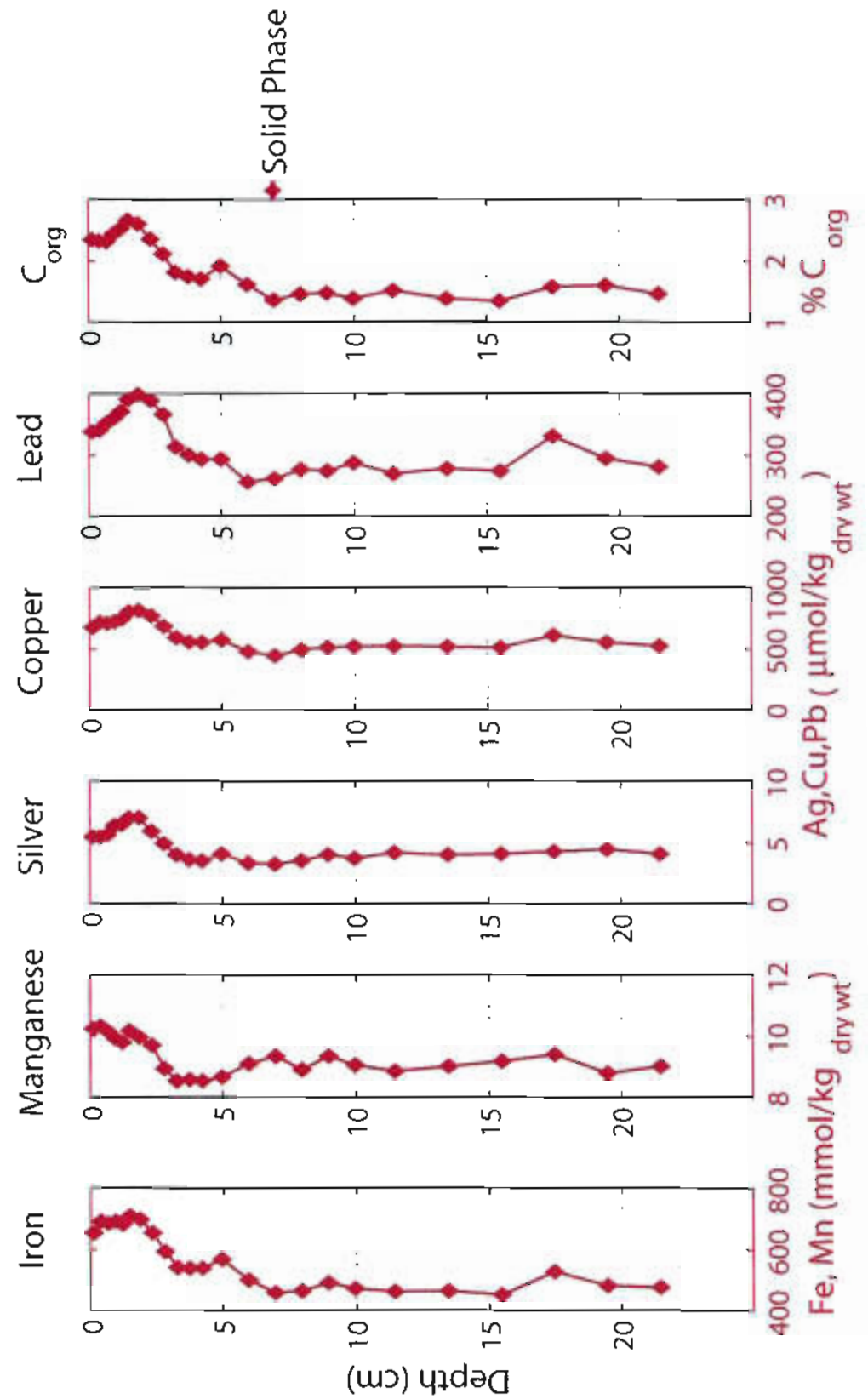

Figure 3-43: Solid phase and porewater profiles of silver, lead, copper, iron, manganese and $\%$ organic carbon for Massachusetts Bay October 2001. 


\title{
Chapter 4
}

\section{Reduced Sulfur Compounds in}

\section{Porewaters}

\begin{abstract}
Porewater profiles of thiol and reduced sulfur compounds have been determined at a strongly reducing site in Boston Harbor, Massachusetts. Derivatization with monobromobimane followed by reverse phase high performance liquid chromatography and fluorescence detection have been used to detect at least six thiols in the porewaters. Maximum individual thiol concentrations of up to $20 \mu \mathrm{M}$ (glutathione equivalents) were either coincident with the sulfide maximum or showed an increase with depth. Tentative identification of some of the chromatogram peaks indicate that 3-mercaptopropanoic acid is the major porewater thiol. Polysulfide species and glutathione are also detected. The polysulfide compounds are shown to be important in controlling the concentration of dissolved copper in anoxic porewaters. The profiles of at least four compounds, including glutathione and polysulfide species indicate a flux to the overlying water. The flux of these compounds calculated from porewater gradient suggests that the sediments are a potentially important source of strong ligands to the waters of Boston Harbor.
\end{abstract}

\subsection{Introduction}

Thiols are organic compounds containing an -SH moiety. The SH functional group of thiols and inorganic reduced sulfur species such as sulfide and polysulfides are easily polarizable and so form strong complexes with soft metals such as silver, lead, mercury and copper(I). The binding constants for these metals with reduced sulfur groups are several orders of magnitude greater than with other functional groups found in aquatic systems [Smith et al., 2002]. Even low concentrations of these species can thus be important in controlling the speciation of metals. 
The sources of thiols in coastal waters arè not well constrained. Most studies have focused on the biological production of thiols for metal detoxification purposes [Ahner and Morel, 1995; Ahner et al., 2002]. Phytoplankton production is important, with peak dissolved concentrations of up to $6 \mathrm{nM}$ of glutathione reported, that coincide with the chlorophyll a maxima in Galveston Bay [Tang et al., 2000a] and in the North Sea and English Channel [Al-Farawati and Berg, 2001]. Coastal sediments are also potential sources, as it is well established that these reduced sulfur compounds can be formed in anoxic sediments (eg Luther et al. [1986]; Kiene and Taylor [1988]; MacCrehan and Shea [1995]). As well as biological production, abiotic reactions, involving the nucleophilic attack by sulfide and polysulfides on small organic molecules generate thiolic compounds in sediments [Vairavamurthy and Mopper, 1987; Kiene and Taylor, 1988].

Profiles of thiols in coastal water have indicated the possibility of a sedimentary source for thiols [Al-Farawati and Berg, 2001]. However, the magnitude of the thiol flux, seasonal variability, compound type and whether a diffusive or advective flux is even possible are largely unknown. The long term fate of any sediment-derived thiols in oxygenated water is also unknown.

The role sediment derived thiols play in the benthic cycling of metals is also not well defined. The presence of these strong ligands in porewaters can lead to enhanced dissolution of metals. Shea and Maccrehan [1988] demonstrated that cysteine, with concentrations up to $12 \mu \mathrm{M}$ in sediments increased the solubility of copper in Chesapeake Bay sediments. In coastal sediments contaminated with metals, ligand enhanced solubility could be an important mechanism facilitating the diffusion or irrigation of metals across the sedimentwater interface. Complexation may also stabilize the metals against oxidation or adsorption and scavenging. The fate of metals in shallow sediments could thus be strongly impacted by presence of strong sulfur-based ligands.

In this chapter, I describe a new method to analyze thiols in porewaters. Simultaneous measurements of trace metal and thiol concentrations are used to determine the importance of thiols and other reduced sulfur compounds in controlling the metal speciation in the porewaters of coastal sediments. High resolution porewater profiles of thiols are used to calculate the flux of thiols across the sediment-water interface. The flux data are used to assess the importance of sediment derived strong ligands to coastal waters. 


\subsection{Methods}

\subsubsection{Study Areas}

The study site is located in coastal Massachusetts east of the city of Boston (Figure 3-1) in Hingham Bay. It is a fine grained sediment site in $5 \mathrm{~m}$ of water and the water column remains oxygenated throughout the year. The concentrations and cycling of trace metals at the site are described in more detail in Chapter 3.

\subsubsection{Sample Collection and Processing}

Divers using SCUBA collected cores from the shallow Hingham Bay site. Core barrels were polycarbonate with a diameter of $10 \mathrm{~cm}$. The cores were placed on ice immediately after collection and brought back to shore. Cores for porewater and solid phase analysis were sectioned under nitrogen as soon as logistically possible. For Hingham Bay samples the cores were driven back to WHOI and sectioned under nitrogen in a $4^{\circ} \mathrm{C}$ walk-in refrigerator, so that sectioning was complete within 12 hours of sample collection.

Sectioned sediments were centrifuged and the centrifuge vials returned to a nitrogen atmosphere where the porewater was carefully removed and filtered through a $0.45 \mu \mathrm{m}$ acrodisc filter. The filtered porewater was distributed into several aliquots for a number of analyses including trace metals, nutrients, total carbon dioxide, alkalinity, sulfide and sulfate. To ensure that there was no oxidation of the compounds of interest, the subsamples for thiols were derivatized in the glove bag immediately after filtration by the method described below.

Analysis of all porewater species other than thiols is described in detail in Chapter 2. Briefly, silver, copper and lead were determined by cobalt-APDC co-precipitation with isotope dilution mass spectroscopy. Sulfide was determined colorimetrically by the methylene blue method of Cline [1969]. Alkalinity, total carbon dioxide $\left(\mathrm{TCO}_{2}\right)$, nutrients and sulfide concentrations were used to calculate the $\mathrm{pH}$ of each porewater sample as described in Section 3.2.3.

\subsubsection{Thiol Analytical Technique}

The fluorescent labeling method developed by Kosower and Kosower [1987] was modified for use in porewaters. Thiols are derivatized by monobromobimane to give covalently bonded 


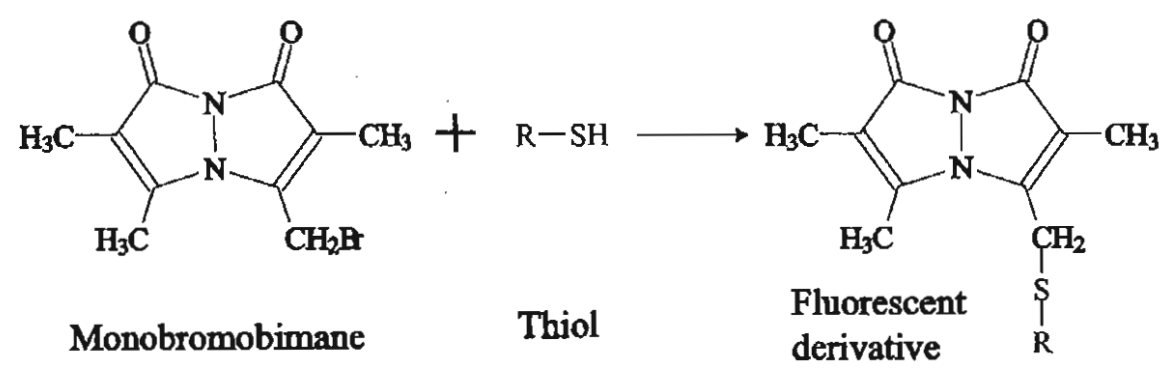

Figure 4-1: Derivatization reaction with monobromobimane

fluorescent adducts that are quantitated using reverse phase high performance liquid chromatography with fluorescence detection [Fahey and Newton, 1987]. This method has been successfully modified for the analysis of phytochelatin in marine algae [Ahner et al., 1995] and trace levels of glutathione in seawater [Tang et al., 2003]. The monobromobimane $(\mathrm{mBBr})$ method proposed for porewaters by Vetter et al. [1987] was modified to ensure consistent derivatization of a large number of porewater samples with a range of $\mathrm{pH}$ values.

The derivatization reaction is $\mathrm{pH}$ dependent. Monobromobimane reacts preferentially with thiols by the general reaction shown in Figure 4-1 in which the $\mathrm{RS}^{-}$is the active nucleophile [Kosower and Kosower, 1987]. Monobromobimane is less reactive towards other nucleophiles such as amines and carboxylates; however if these compounds are found at high concentrations, side reactions can become important. At $\mathrm{pH}$ values greater than 9 the derivatization of amines becomes significant. Maintaining the $\mathrm{pH}$ between 7 and 8 optimizes the derivatization reaction for thiols [Kosower and Kosower, 1987; Vetter et al., 1987]. The $\mathrm{pH}$ values found in the porewaters of Hingham and Massachusetts Bay range from 6.1 to 8.5 with an average of 7.3. The majority of samples thus fall within the optimum range. The method described by Vetter et al. [1987] requires the $\mathrm{pH}$ to be adjusted individually on each porewater sample prior to addition of monobromobimane. This is not a practical approach for collecting thiol data in addition to all the other parameters that are measured on the Hingham Bay porewaters, as treating each sample individually would be too time consuming.

To control the $\mathrm{pH}$ of the samples $50 \mu \mathrm{L}$ of $40 \mathrm{mM}$ borate buffer with $10 \mathrm{mM}$ EDTA adjusted to $\mathrm{pH} 8.2$ was pre-spiked into glass test tubes, followed by $50 \mu \mathrm{L}$ of $10 \mathrm{mg} / \mathrm{mL}$ monobromobimane (Molecular Probes) in acetonitrile. Pre-spiked test tubes were prepared on the morning of sample collection and stored in the dark at $4^{\circ} \mathrm{C}$ until the centrifuged 
samples were available for filtration. The test tubes were then transferred into the glove bag. In the glove bag each porewater sample was filtered into a $10 \mathrm{ml}$ plastic vial (Evergreen Industries) and $500 \mu \mathrm{L}$ of the porewater sample was pipetted into the pre-spiked test tubes. The samples were then stored at $4^{\circ} \mathrm{C}$ overnight and then frozen at $-40^{\circ} \mathrm{C}$ until analyzed by HPLC. For standard addition calibrations, pre-prepared thiol stock solutions were added into either porewater samples or synthetic seawater samples and then $500 \mu \mathrm{L}$ of the spiked sample was added to the pre-spiked test tubes. $500 \mu \mathrm{L}$ of synthetic seawater was also added into the pre-spiked test tubes to act as a reagent blank.

The concentration of monobromobimane was chosen to ensure at least a 2 times excess of probe over the maximum sulfide concentration, as recommended by Vetter et al. [1987]. The buffer concentration was chosen to ensure that any samples below $\mathrm{pH} 7$ were brought up to a higher $\mathrm{pH}$ as reagent peaks dominated the chromatogram below this value. From the measurements of Shea and Maccrehan [1988], individual thiol concentrations in the $\mu \mathrm{M}$ range were anticipated. Interference from class $\mathrm{B}$ metals that strongly complex thiols such as silver, copper and lead, found at concentrations below $50 \mathrm{nM}$ in the Hingham Bay sediments, were not expected to be significant. The concentration of dissolved $\mathrm{Fe}$ (II) in the porewaters could reach up to $200 \mu \mathrm{M}$, and this concentration was found to strongly suppress the fluorescent signal. Inclusion of EDTA in the buffer solution solved this problem. Fahey and Newton [1987] found that samples stored between 4 and $-70^{\circ} \mathrm{C}$ were stable for up to 20 months, so that the delay between sample collection and analysis should not have led to any sample degradation. Replicate samples analyzed a year apart in this study did not show any significant changes when stored at $-40^{\circ}$. However, samples stored at $4^{\circ}$ for a month did show changes in intensities, indicating that porewaters may not be as stable as the samples investigated by Fahey and Newton [1987].

A Hewlett Packard HPLC system was used to separate the thiol compounds. The system consisted of an inline solvent degasser (HP 1322A), binary pump (HP 1312A) and an autosampler (HP 1100) and was controlled by Hewlett Packard software. The reverse phase column was an Alltech C-18 $5 \mu \mathrm{m}$ Solvent Miser $250 \mathrm{~mm}$ column equipped with a C-18 guard column. Mobile phase A was $1 \%$ acetonitrile in $100 \mathrm{mM}$ ammonium acetate solution and mobile phase $B$ was pure acetonitrile. The elution gradient was as follows $0 \%$ $\mathrm{B}$ for 5 minutes, a linear increase to $30 \% \mathrm{~B}$ over 35 minutes, then a linear increase to $100 \% \mathrm{~B}$ over 10 minutes, 10 minutes at $100 \% \mathrm{~B}$ to flush the column, a linear decrease to 


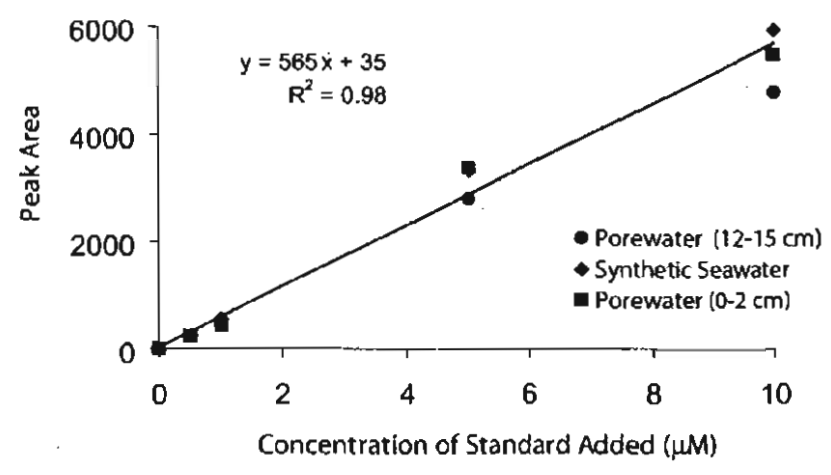

Figure 4-2: Comparison of calibration curves for mercaptosuccinic acid spiked into both synthetic seawater and a porewater sample from Hingham Bay October 2003 depth 0-2 cm

$0 \% \mathrm{~B}$ over 5 minutes and a final 20 minutes at $0 \% \mathrm{~B}$ to re-equilibrate the column. The flow rate was $0.45 \mu \mathrm{l} / \mathrm{min}$. The injection volume was $20 \mu \mathrm{L}$ but was increased if there was sufficient sample available. The thiols were quantified post column with a Hewlett Packard (HP 1321A) Aluorescence detector with an excitation wavelength of $390 \mathrm{~nm}$ and an emission wavelength of $475 \mathrm{~nm}$. Porewater samples were filtered through a $0.2 \mu \mathrm{m}$ polysulfone membrane (Pall-Gelman) acrodisc filter prior to loading into the autosampler.

Thiol compounds, glutathione (98\%), L-cysteine (as L-cysteine hydrochloride), 3-mercaptopropanoic acid, 2-mercaptopropanoic acid, allyl mercaptan (70\%), mercaptoacetic acid (98\%), mercaptosuccinic acid (99\%), 2-mercaptoethanol, ethanethiol (99\%) and $\gamma$ glutamylcysteine were purchased from Fisher Scientific. Standard solutions were prepared in deoxygenated synthetic seawater and stored under nitrogen for up to 24 hours until they were spiked into porewaters or synthetic seawater samples. Additional compounds that were also derivatized to assess their retention times were sodium sulfite, sodium thiosulfate and sodium sulfide.

Calibration curves were linear over the range $0-20 \mu \mathrm{M}$ and, as shown in Figure 4-2, the calibration curve for standards spiked into synthetic seawater and porewaters from two different redox zones were very similar. This indicates that the porewater matrix was not significantly interfering with the derivatization or fluorescence. Thiols were calibrated with the calibration curve from the appropriate redox zone. 


\subsection{Results and Discussion}

\subsubsection{Derivatization Results}

There were distinct changes in the chromatograms with depth in the sediment column. A typical chromatogram from a porewater is shown in Figure 4-3. The profile from a reagent blank is also shown for comparison. A porewater spiked with known thiols is also shown in Figure 4-4. The dominant peaks are labeled in Figure 4-3 and the variation in these peaks with depth is shown in Figure 4-6 for September 2002 and Figure 4-7 for October 2003. For October 2003, due to a larger porewater volume available, a larger HPLC injection volume was possible so that lower intensity peaks could be detected. The scale on Figure 4-3 is expanded in Figure 4-5 to show the detail of some of the minor peaks that were consistently observed in the sediment profile. The profiles of these minor peaks are shown in Figure 48. The identities of many of the peaks remain unknown, so the data shown in the above profiles are converted from peak area to concentration assuming the calibration curve for glutathione. Glutathione was selected as the model compound as it elutes in the middle of the chromatogram and its concentration is accurately known due to its stability when refrigerated. Other smaller thiol compounds have a tendency to oxidize over time so are less suitable for a general calibration as they would require regular standardization.

The chromatogram does extend beyond 40 minutes, however the baseline drifted and was noisy beyond this point. The intensities of peaks in this region were thus variable. Attempts to regenerate the column by flushing the column with the Alltech recommended series of solvents did not improve the situation. Peaks in this region were not quantified. Derivatized sulfide eluted in this region as a very broad peak. Monobromobimane could be a good way of determining trace levels of sulfide, however a chromatographic method more focused on sulfide than this method is certainly required.

\section{Peak Identification}

In order to identify the compounds in the chromatograms, readily available thiols that had been previously detected in porewaters by Mopper and Delmas [1984]; Vairavamurthy and Mopper [1990]; Shea and Maccrehan [1988] were added into porewaters from a range of depths to assess co-elution. Co-elution is a necessary but not sufficient criterion for positive identification of a peak. Only three of the selected compounds co-eluted with the porewater 


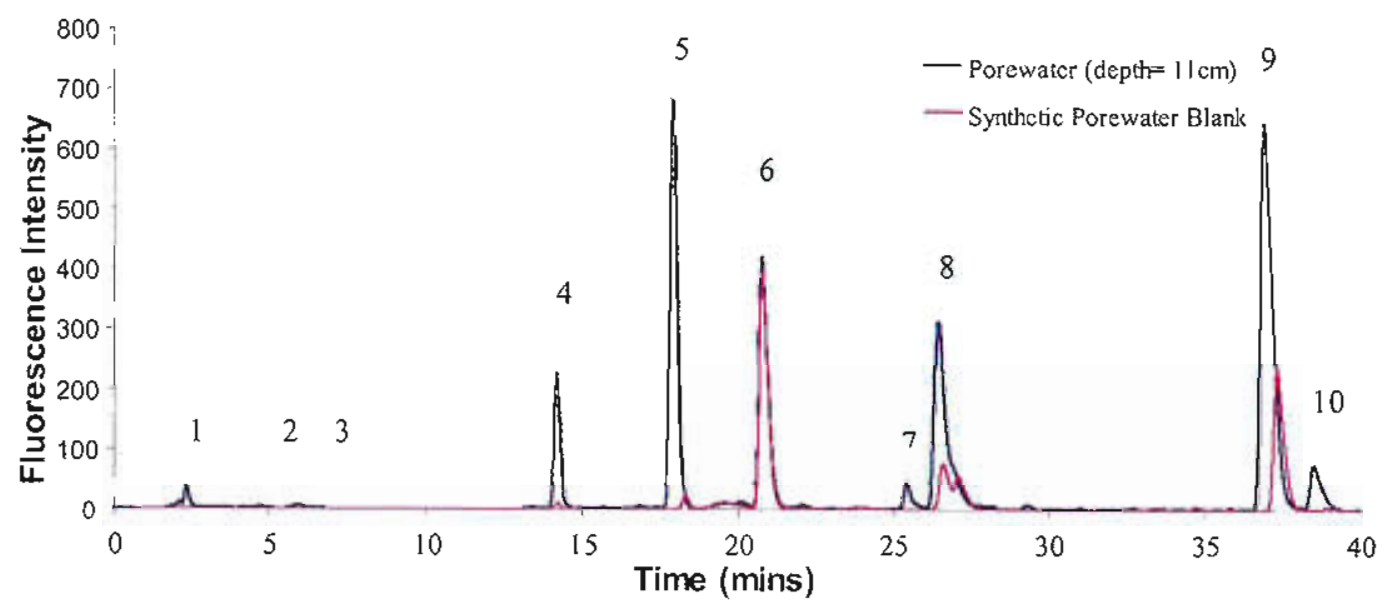

Figure 4-3: Chromatogram from a derivatized Hingham Bay porewater sample. A porewater from a depth of $11 \mathrm{~cm}$ is shown as well as a reagent blank.

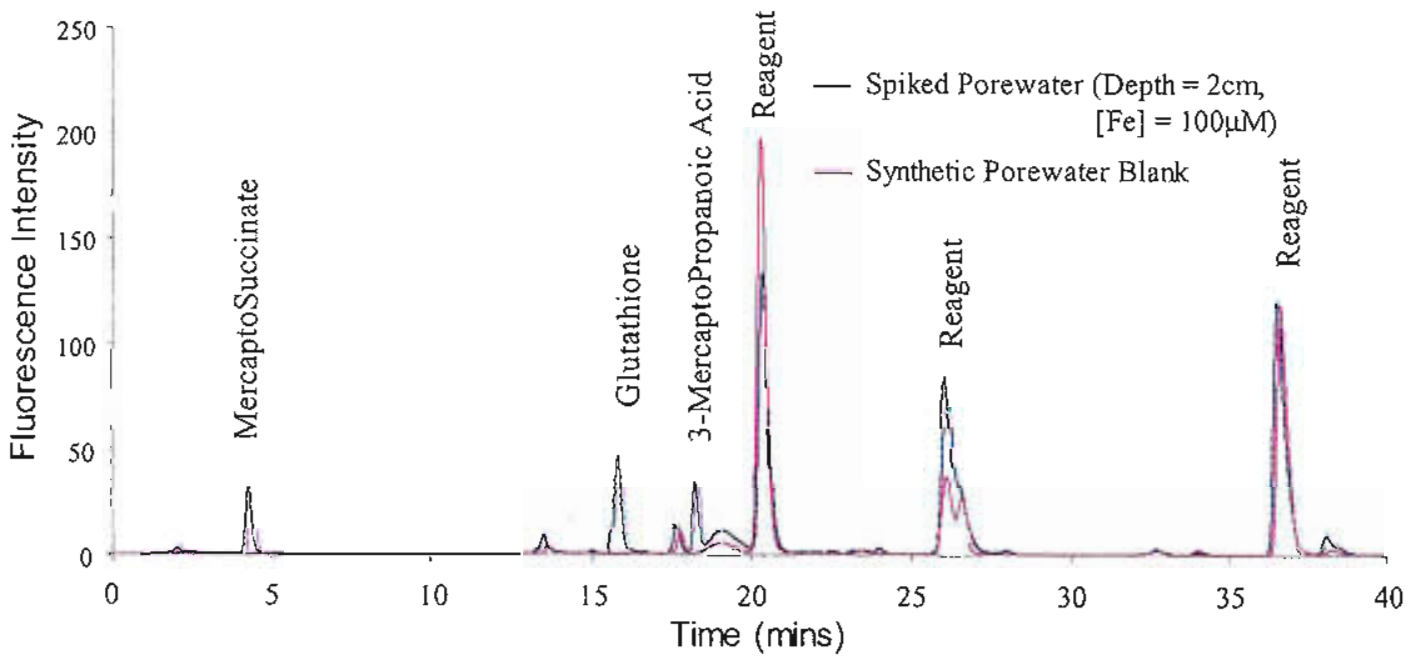

Figure 4-4: A Hingham Bay porewater spiked with mercaptosuccinic acid, glutathione and 3-mercaptopropanoic acid to give a spiked concentration of approximately $1 \mu \mathrm{M}$. 


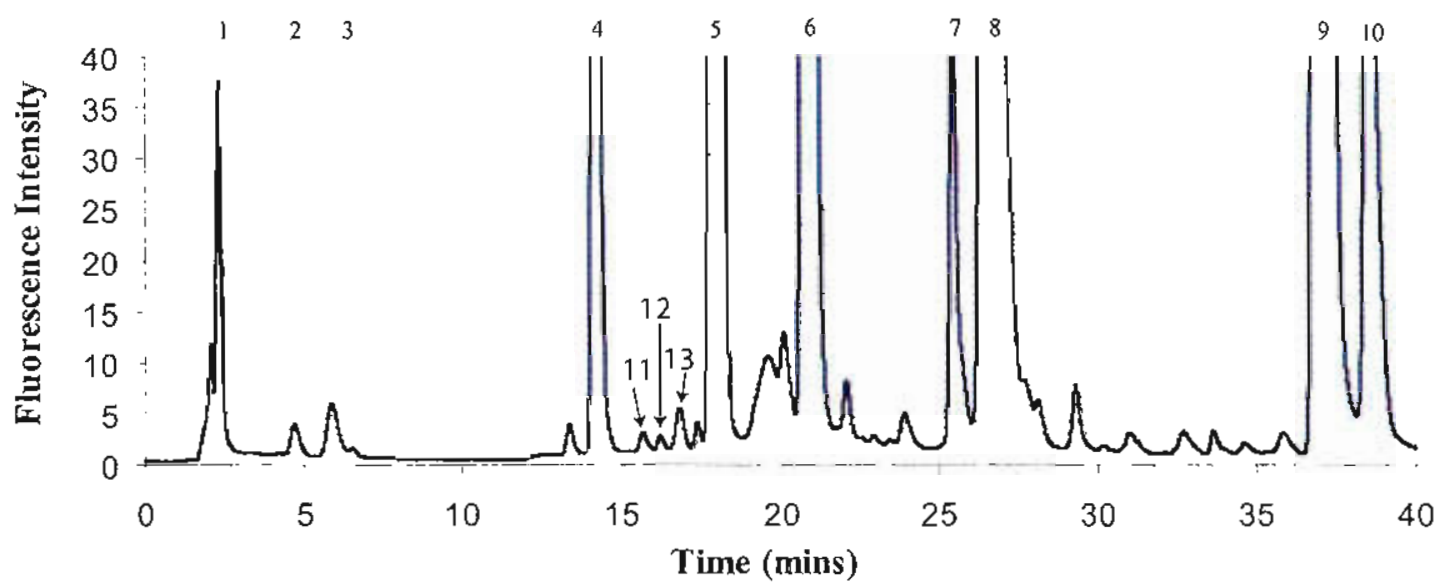

Figure 4-5: The chromatogram of the Hingham Bay porewater from Figure 4-3 at an expanded scale to show the minor peaks.
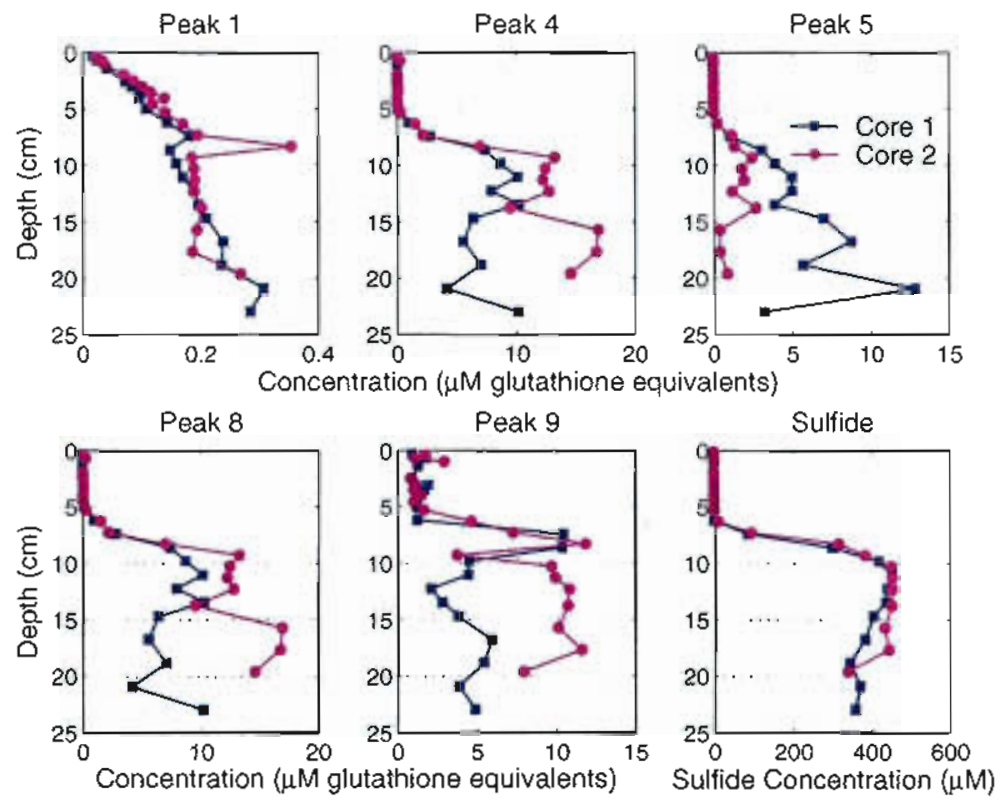

Figure 4-6: Profiles of the dominant fluorescent derivatives for Hingham Bay September 2002 as well as the sulfide data determined by the methylene blue UV spectroscopy method of Cine [1969]. The peak numbering is with reference to the labeled peaks in Figure 4-3. Tentative assignment of peaks is peak $5=3$-mercaptopropanoic acid, peaks 8 and $9=$ polysulfide species. 

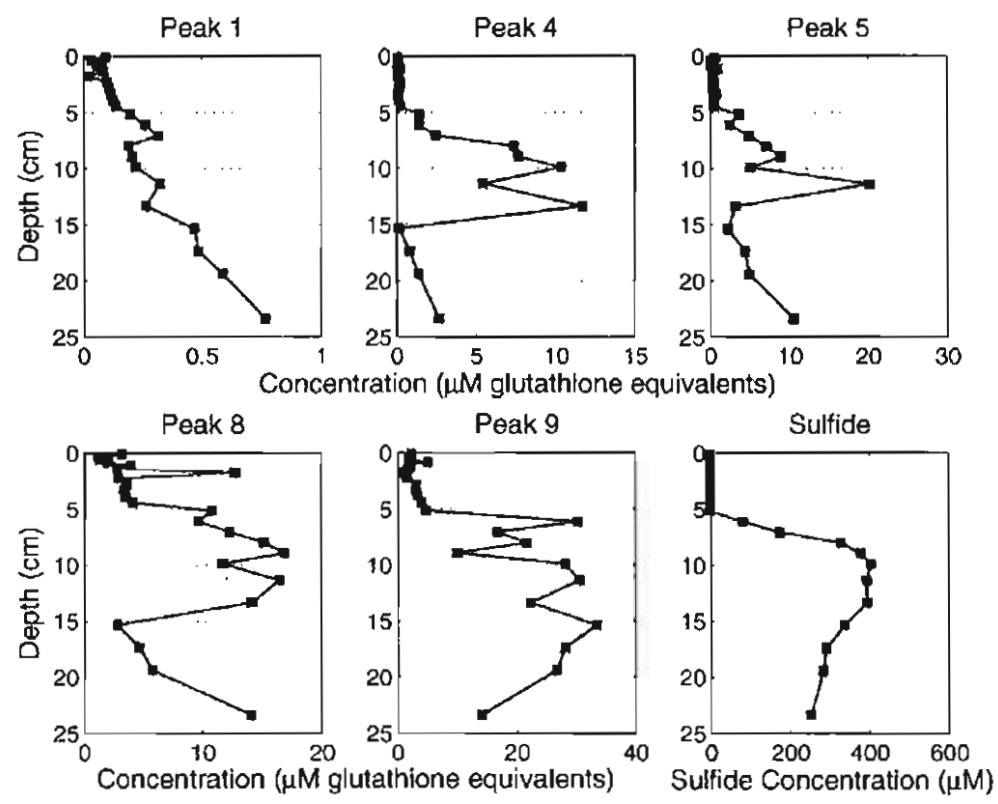

Figure 4-7: Profiles of the dominant fluorescent derivatives for Hingham Bay October 2003 as well as the sulfide data determined by the methylene blue UV spectroscopy method of Cline [1969]. The peak numbering is with reference to the labeled peaks in Figure 4-3. Tentative assignment of peaks is peak $5=3$-mercaptopropanoic acid, peaks 8 and $9=$ polysulfide species.
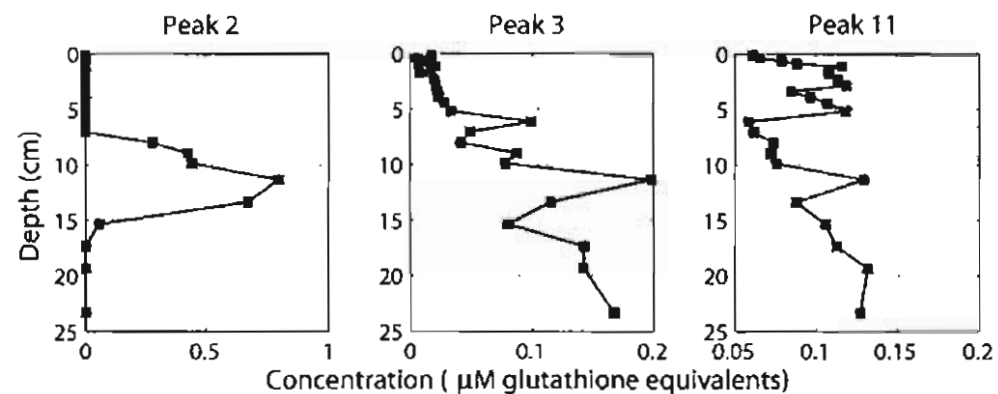

Peak 12

Peak 13
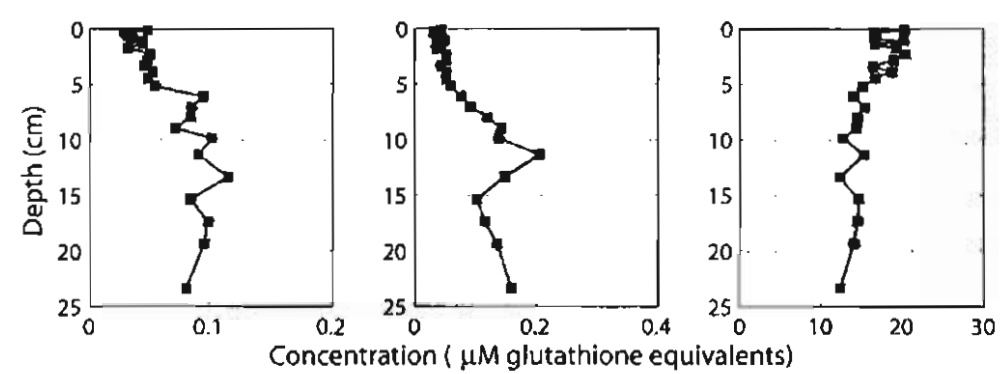

Figure 4-8: Profiles of the minor fluorescent derivatives for Hingham Bay October 2003. Peak number 6 is a reagent peak. Tentative assignment of peaks is peak $2=$ mercaptosuccinic acid peak 11 = glutathione. 
peaks. The tentative identification of peak 2 is mercaptosuccinate $\left(\mathrm{HOOCCH}_{2} \mathrm{CH}(\mathrm{SH}) \mathrm{COOH}\right)$, peak 5 is 3-mercaptopropanoic acid $\left(\mathrm{CH}_{2}(\mathrm{SH}) \mathrm{CH}_{2} \mathrm{COOH}\right)$ and peak 11 is glutathione ( $\mathrm{HOOCCH}\left(\mathrm{NH}_{2}\right) \mathrm{CH}_{2} \mathrm{CH}_{2} \mathrm{CONHCH}\left(\mathrm{CH}_{2} \mathrm{SH}\right) \mathrm{CONHCH}_{2} \mathrm{COOH}$ ). Peak 5 is a broad peak that co-elutes with 3-MPA, but there may be additional co-eluting compounds in peak 5 that could not be resolved. 3-MPA concentrations may thus be overestimated. Additional chromatography is required to determine this. There are some additional compounds such as mercaptopyruvate [Shea and Maccrehan, 1988] and methanethiol [Mopper and Delmas, 1984] that should also be derivatized so their retention times can be compared to the porewater samples. Further verification, including running the spiked and unspiked samples on a different column, is required to confirm the identity of these peaks. Liquid chromatographymass spectroscopy (LC-MS) separation and analysis will also provide possible clues to the identity of the unknown peaks and help confirm the identity of the tentatively assigned peaks.

Polysulfides, $\mathrm{S}_{x} \mathrm{~S}^{2-}$, are intermediates in the oxidation of sulfide and have been detected electrochemically in salt marsh [Luther et al., 1985] and estuarine sediments [Rozan et al., 2000b]. Polysulfides are strong nucleophiles [Schwarzenbach et al., 2003] and are also likely to react with monobromobimane. As a preliminary investigation to assess if monobromobimane derivatives of polysulfides could be detected, the method used by Chen and Gupta [1973] to prepare aqueous polysulfides was employed. This involved the reaction of an elemental sulfur suspension with dissolved sodium sulfide. The progress of the reaction was monitored with UV-VIS spectroscopy at $290 \mathrm{~nm}$. Samples of the suspension were first filtered to remove remaining sulfur and then derivatized with monobromobimane. Broad peaks at average retention times of 26.5 and 37.3 minutes, as well as a peak at 20.1 minutes, were recorded. The peak at 20.1 minutes is a thiosulfate peak. Thiosulfate is the oxidation product of polysulfides [Chen and Gupta, 1973], so detection of thiosulfate indicates the dynamic nature of polysulfide formation.

Chromatograms of porewaters from below the sulfide interface also have peaks close to 26.5 and 37.3 minutes, so peak numbers 8 and 9 of Figure 4-3 are tentatively assigned to be polysulfides, by comparison of retention times. Unfortunately it is not possible to determine which polysulfide species are present from the reaction of sulfur and sulfide. The UV adsorption maxima of $\mathrm{S}_{4}^{2-}$ and $\mathrm{S}_{5}^{2-}$ overlap within $7 \mathrm{~nm}$ [Giggenbach, 1972] so are difficult to separate. Polysulfide reactions proceed rapidly, so polysulfide ions of different 
chain lengths will always co-exist in aqueous solution [Shea and Helz, 1988]. Purchasing polysulfide compounds thus does not solve the problem as when they are prepared in an aqueous solution the species composition changes. Electrochemical methods [Rozan et al., $2000 \mathrm{~b} ;$ Luther et al., 2001] report polysulfides as $\mathrm{S}_{x}^{2-}$. The two peaks may be different polysulfide species; however the peaks are broad and the possibility of double labeling of one polysulfide ion by two $\mathrm{mBBr}$ molecules also arises so that the broad peaks may actually be due to the co-elution of a number of different compounds. Mass spectrometry on the Huorescent derivatives could resolve this, not only confirming the identity of these peaks but also determining the relative abundances of each polysulfide species.

The dominant thiols found in porewaters of non salt marsh sediments by others are 3-mercaptopropanoic acid (3-MPA), cysteine and glutathione [Mopper and Delmas, 1984; Shea and Maccrehan, 1988; Kiene and Taylor, 1988; Vairavamurthy and Mopper, 1990; MacCrehan and Shea, 1995]. The concentrations of 3-MPA (Peak 5) found by Shea and Maccrehan [1988] and [MacCrehan and Shea, 1995] in Chesapeake Bay reached up to $10 \mu \mathrm{M}$, similar to the levels observed here, while in Biscayne Bay, 3-MPA concentrations only reached $0.2 \mu \mathrm{M}$ [Kiene and Taylor, 1988]. Glutathione concentrations (Peak 11) of up to 0.2 $\mu \mathrm{M}$ were reported in Biscayne Bay sediments [Kiene and Taylor, 1988]. In the Chesapeake Bay the glutathione concentrations reached $5 \mu \mathrm{M}$, but this peak value was highly seasonal and concentrations of less than $1 \mu \mathrm{M}$ were reported from October to June. Glutathione concentrations at Hingham Bay are lower than those reported, reaching a maximum of only $0.14 \mu \mathrm{M}$.

Cysteine is reported to reach levels of up to $12 \mu \mathrm{M}$ in the sediments of Chesapeake Bay, yet is not detected in the sediments of Hingham Bay. This discrepancy may be because the method described here, like that of [Kiene and Taylor, 1988], only measures free thiols. At $\mathrm{pH}$ values above neutral, cysteine $\left(\mathrm{HSCH}_{2} \mathrm{CH}\left(\mathrm{NH}_{2}\right) \mathrm{COOH}, \mathrm{CySH}\right)$ auto-oxidizes to cystine [Jocelyn, 1972]:

$$
2 \mathrm{CySH} \rightarrow \mathrm{CySSCy}+2 \mathrm{H}^{+}+2 \mathrm{e}^{-}
$$

Disulfides such as cystine will not react with monobromobimane and so are not detected. In the reducing sediments of Hingham Bay, oxidized forms of the thiols were not expected. However, iron(II) and copper(II) have a strong catalytic effect on cysteine oxidation $\mid$ Jocelyn, 1972$]$ and so may be responsible for maintaining any dissolved cysteine in an oxidized 
form. Glutathione is also readily oxidized to a disulfide, and in the absence of metal catalysts reacts at a similar rate to cysteine. However, free glutathione is detected in the sediments probably because glutathione oxidation is not as strongly affected by the presence of metals. At a thiol:metal molar ratio of 1:0.01, the oxidation rate of glutathione increases by a factor of 8 , while the rate for cysteine increase by a factor of 53 [Jocelyn, 1972]. In Hingham Bay the concentration of iron(II) is in excess compared to the thiol concentration, so the oxidation of cysteine may be even more accelerated and account for the absence of cysteine in the porewaters. In order to determine the concentrations of oxidized cysteine in these porewaters, addition of a reducing agent such as dithiothrietol [Ahner et al., 1995] or tri-n-butylphosphine [Tang et al., 2000b] prior to monobromobimane addition seems to be necessary.

\section{Sediment Profiles}

Irrespective of identity, most of the profiles show a close correspondence with sulfide. All of the compounds that reach concentrations above $1 \mu \mathrm{M}$ are generated within the sulfidic zone. This obvious link to sulfide helps to confirm that the compounds derivatized are in fact reduced sulfur compounds and not amines or carboxylates, as these compounds would be unlikely to show such a distinct relationship with the sulfide profile. Subtle differences between the thiol profiles reflect the different mechanisms of formation and consumption of each compound.

In Biscayne Bay Kiene and Taylor [1988] found that glutathione peaked above the maximum in sulfide, and this is similar to the profile observed here. Glutathione (the tripeptide $\gamma$-glutamylcysteinylglycine) is the most abundant non-protein thiol found in cells, and its primary biological function is to act as a non-enzymatic reducing agent [Jocelyn, 1972]. The profile of glutathione (peak 11) has a maximum in the sub-oxic zone and is distinctly different from that of all other peaks, suggesting a different source. The glutathione may simply be present due to cell lysis or exudation by microorganisms that are present in the sub-oxic zone. Kiene and Taylor [1988] suggest the glutathione in their porewaters may be associated with aerobic organisms, though the peak in glutathione in both Hingham Bay and Biscayne Bay occurs in sub-oxic sediments. MacCrehan and Shea [1995] suggest that the presence of glutathione in porewaters may be due to the microbial solubilization of elemental sulfur or due to its use as a cofactor for extracellular $S^{\circ}$ enzyme activity. It 
is not possible to assess if this is the case from a porewater profile, especially as elemental sulfur was not measured. However although the elemental sulfur and glutathione peaks in Chesapeake Bay are co-incident, this may be due to the highly reducing sediments and compressed redox zones (sulfide is measured in the $0-2 \mathrm{~cm}$ porewater sample) at this site rather than the microbiological link they propose. Hingham Bay, with a sub-oxic zone of at least $5 \mathrm{~cm}$, may be a more appropriate location to measure elemental sulfur and thus assess if glutathione is playing an extra-cellular role in sulfur geochemistry.

The 3-mercaptopropanoic acid profile (Peak 5) reaches a maximum below the sulfide interface, as in Biscayne Bay [Kiene and Taylor, 1988]. In Hingham Bay in both years the concentration seems to build up below the sulfide interface more slowly than the other major peaks. This may be because two of the other major peaks have been tentatively identified as polysulfides and are formed by the reaction of $\mathrm{HS}^{-}$and elemental sulfur, both of which are tightly linked to the sulfide profile. Production of thiols within the sediments requires precursor organic molecules in addition to inorganic sulfur compounds, so this may account for the different profile shapes. 3-MPA has been shown to be formed abiotically in sediments by the Michael addition of $\mathrm{HS}^{-}$to the double bond of acrylate $\left(\mathrm{CH}_{2}=\mathrm{CHCOOH}\right)$ [Vairavamurthy and Mopper, 1987] as well as due to biotransformations of sulfur-containing amino acids [Kiene and Taylor, 1988; Kiene, 1991]. As the generation of 3-MPA from amino acids does not require the presence of sulfide, the strong relationship between the 3-MPA peak and the sulfide profile suggests that the abiotic mechanism is more likely in this case.

The profiles of the peaks tentatively assigned to be polysulfides (peaks 8 and 9 ) increase rapidly with sulfide, reaching maxima at shallower depths than the sulfide maxima. Peak 9 shows a very sharp peak in the region in which sulfide is increasing in each profile. Polysulfides were shown to form in the region in which sulfide was decreasing in a microbial mat [Luther et al., 2001] and in the sediments of Chesapeake Bay [Shea and Maccrehan, 1988] so the profiles in Hingham Bay are consistent with these observations. In Chesapeake Bay, total polysulfides reached $250 \mu \mathrm{M}$, while in the microbial mat the concentrations reached 40 $\mu \mathrm{M}$. These are both very different environments from Hingham Bay, but the approximate concentrations measured here, assuming a glutathione calibration is appropriate, fall within the range of previously observed concentrations. One of the most interesting features of the polysulfide peaks is that although they have considerably reduced concentrations above the sulfide interface, measurable concentrations persist up to the sediment-water interface. 
Polysulfides, although unstable, oxidize slowly [Giggenbach, 1974]. These compounds can thus resist oxidation to some extent and are likely to be important ligands in the suboxic region where sulfide concentrations are low. The profiles of these compounds suggest they are fluxing upwards, and as there is a measurable concentration at the sediment-water interface a flux into the water column is possible.

Most of the minor peaks in Figure 4-8 also show a Alux upwards towards the interface, and a flux into the water column is also indicated. The fluxes are quantified in the following section.

\subsubsection{Sediments as a Source of Reduced Sulfur Compounds}

The porewater profiles indicate that there is a flux of these compounds out of the sediments. In order to quantify the fluxes by Equation 3.7, an estimate of the diffusion coefficient $D$ is required. As the identity of most of the peaks that have a non-zero concentration at the interface is currently unknown or tentative, this is not possible to do accurately. However, assuming that peaks 8 and 9 are polysulfides, that $S_{4}^{2-}$ and $S_{5}^{2-}$ are the dominant species in porewaters [Luther et al., 1986] and that peak 5 and 11 are 3-MPA and glutathione respectively, the empirical expression derived for organic compounds and gases up to a molecular weight of $342 \mathrm{~g} / \mathrm{mol}$ that [Schwarzenbach et al., 2003]

$$
D_{w}=2.7 \times 10^{-4} m^{-0.71}
$$

where $D_{w}$ is the diffusion coefficient in water $\left(\mathrm{cm}^{2} / \mathrm{s}\right)$ and $m$ is the molecular mass $(\mathrm{g} / \mathrm{mol})$. is used to approximate the diffusion coefficient of peaks 5, 8, 9 and 11. The porewater concentrations in the upper porewater sample and the calculated fluxes for the compounds which have been identified are given in Table 4.1. The flux calculation assumes that the gradient can be represented by the difference in concentration between the upper porewater sample and the overlying water $(\Delta z=1.5 \mathrm{~mm})$. The overlying water concentration is assumed to be similar to the concentrations found in Galveston Bay (0.2-6 nM glutathione). An average of these values is taken for the overlying water glutathione concentration. For polysulfides and 3-MPA, assuming they form part of the strong ligand pool, an overlying water concentration of $10 \mathrm{nM}$ is selected based on the work of Moffett et al. [1997]. As the concentration of polysulfides and 3-MPA is on the order of $\mu \mathrm{M}$ the selection of the 
Table 4.1: Diffusive flux across the sediment-water interface of reduced sulfur compounds

\begin{tabular}{|c|c|c|c|c|c|c|}
\hline $\begin{array}{l}\text { Peak number } \\
\text { Proposed Compound }\end{array}$ & $\begin{array}{c}1 \\
\text { unknown }\end{array}$ & $\begin{array}{c}5 \\
3-\mathrm{MPA} \\
\end{array}$ & $\begin{array}{c}8 \\
\text { polysulfide }\end{array}$ & $\begin{array}{c}9 \\
\text { polysulfide }\end{array}$ & $\begin{array}{c}11 \\
\text { glutathione }\end{array}$ & $\begin{array}{r}12 \& 13 \\
\text { unknown }\end{array}$ \\
\hline $\begin{array}{l}\text { Porewater conc }(\mu \mathrm{M})^{1} \\
D_{w}\left(\mathrm{~cm}^{2} / \mathrm{s}\right)^{2} \\
\text { Flux }\left(\mathrm{mmol} / \mathrm{m}^{2} / \mathrm{yr}\right) \\
\text { Calculated Harbor Conc }\end{array}$ & 0.1 & $\begin{array}{c}0.4 \\
1 \times 10^{-5} \\
7 \\
4-13\end{array}$ & $\begin{array}{c}3 \\
7-9 \times 10^{-6} \\
40 \\
22-90\end{array}$ & $\begin{array}{c}2 \\
7-9 \times 10^{-6} \\
30 \\
16-66\end{array}$ & $\begin{array}{c}0.06 \\
5 \times 10^{-6} \\
0.5 \\
0.3-0.9\end{array}$ & 0.05 \\
\hline
\end{tabular}

1 October 2003 0-3 mm sample

2 Estimated from Equation 4.2 and assuming Peaks 8 and 9 are either $\mathrm{S}_{5}^{2-}$ or $\mathrm{S}_{4}^{2-}$

${ }^{3}(\mathrm{nM})$ calculated assuming steady state using Equation 4.5. The given range is due to the range in residence times and diffusion coefficients

overlying water concentration has a negligible effect on the calculated flux considering the measurement uncertainties. If there is oxidation right at the sediment-water interface, removing these species from the porewaters on scales smaller than the sampling resolution, the diffusive flux will be overestimated. As the concentrations of the polysulfides have been calibrated with a glutathione calibration curve, and the molecular weight of the species in the polysulfide peaks is unknown Table 4.1 only represents a rough estimate of potential fluxes.

To assess if the flux from the sediments of these reduced sulfur compounds could be an important contributor to the strong ligand pool in Boston Harbor a very simple box model of Boston Harbor, is used. Strong ligands are known to be biologically produced [Moffett and Brand, 1996; Rue and Bruland, 1997] or derived from terrestrial humic substances [Kogut and Voelker, 2001]. If, however, for the purpose of assessment we assume that the sediments are the dominant source of strong ligands and that the ligands do not oxidize or otherwise degrade, then assuming steady state, the input of thiols from the sediments will equal the removal of thiols due to flushing (assuming also that the concentrations of thiols in the incoming ocean water is negligible). ie Input $_{\text {sed }}=$ Output $_{\text {flushing }}$ The output due to fiushing can be approximated by the product of the ligand concentration $C$ in the water column and the net flow rate of water from the Harbor, $Q_{n e t}$ :

$$
\text { Output }_{\text {flushing }}=C Q_{\text {net }}
$$


Knowing that the residence time, $\tau_{\text {res }}$ in the Harbor ranges between 3 and 10 days $\mid$ Signell et al., 2000] and is driven mainly by tidal exchange, $Q_{\text {net }}$ can be approximated by

$$
\tau_{\text {res }}=V_{\text {harbor }} / Q_{\text {net }}
$$

so that the concentration expected in the waters of Boston Harbor is given by

$$
C=F \times(\text { sediment area }) * \tau_{\text {res }} / V_{\text {harbor }}
$$

Approximating the volume of the Harbor, $V_{\text {harbor }}$ as the product of the Harbor area (125 $\left.\mathrm{km}^{2}\right)$ and average depth (4.9 $\mathrm{m}$ [Signell et al., 2000]) and assuming that the Hingham Bay site represents $41 \mathrm{~km}^{2}$ of the Harbor area (pers comm M. Bothner, USGS) the concentrations can be approximated from the calculated porewater flux, $F$ and are also given in Table 4.1.

For glutathione, concentrations on the order of $0.1-1 \mathrm{nM}$ are predicted. No measurements of glutathione in the waters of Boston Harbor have been made; however Tang et al. [2000a] reported glutathione concentrations of 0.2 to $6 \mathrm{nM}$ in the waters of Galveston Bay and Al-Farawati and Berg [2001] reported unidentified thiols in the concentration range of 0.7 to $4 \mathrm{nM}$ in the waters of the North Sea and English channel. This simple box model has too many assumptions to make a definitive statement, but the preliminary results suggest that the sediments in Boston Harbor could be an important source of glutathione to the water column.

Polysulfides are strong copper ligands. The equilibrium constant for the reaction

$$
\mathrm{Cu}^{2+}+\mathrm{S}_{5}^{2-}+\mathrm{HS}^{-} \rightleftharpoons \mathrm{CuS}\left(\mathrm{S}_{5}^{2-}\right)+\mathrm{H}^{+}
$$

is $10^{18.3}$ [Shea and Helz, 1988] at an jonic strength of $0.5 \mathrm{M}$. So taking a seawater $\mathrm{pH}$ of 8 and and a trace level of bisulfide of $10^{-9} \mathrm{M}$ gives $K_{\text {cond }}$ (polysulfide) $\sim 10^{17}$. Concentrations of operationally defined strong copper ligands in Massachusetts coastal waters was found to be up to $10 \mathrm{nM}$ by Moffett et al. [1997] and 1-18 nM by Kogut and Voelker [2003]. Polysulfides would form part of this pool, so the concentrations of polysulfides predicted in the waters of Boston Harbor are too high. This is not surprising, as it is unrealistic to assume no oxidation of such unstable species [Shea and Helz, 1988]. However, the kinetics of polysulfide 
oxidation has been shown to be extremely slow, and laboratory solutions are stable with respect to disproportionation for up to a year [Giggenbach, 1974]. In the environment metal catalysis, hydrogen peroxide and biological catalysis may considerably enhance this rate. However, the calculation suggest that even if only $1 \%$ of the compounds measured in the surface porewaters resist oxidation in the overlying waters, it will mean the sediments are a potentially important source of strong ligands to the Harbor. Glutathione, 3-MPA, polysulfides and the other three unidentified peaks may be the strong ligands measured by Skrabal et al. [1997] and Shank et al. [2004] to be fluxing from incubated Chesapeake Bay and Cape Fear Estuary sediments. In order to definitively conclude that the sediments are a significant source, there are several key assumptions that need to be assessed further. The diffusive porewater flux calculated from the porewater profiles needs to be compared with samples collected by a benthic chamber to ensure the flux calculated is valid. The rate of oxidation of these sediment-derived compounds, once they reach the overlying water, also needs to be investigated to fully understand the importance of these compounds in oxygenated waters.

\subsubsection{Trace Metal Speciation in Porewaters}

The speciation of metals in anoxic environments has been investigated from a thermodynamic perspective by a number of authors [Jacobs and Emerson, 1982; Boulegue et al., 1982; Shea and Maccrehan, 1988]. Due to limitations in available thermodynamic data and the difficulty in identifying the correct solid phase, these calculations have not been entirely successful in explaining measured metal concentrations. The measured concentrations of dissolved metals generally exceed those predicted from thermodynamic models [Shea and Maccrehan, 1988]. By including thiols and polysulfides in speciation calculations on porewaters, Shea and Maccrehan [1988] were able to determine that cysteine complexes as well as polysulfide complex were important copper species. With the inclusion of reduced sulfur species in a thermodynamic model the estimated copper concentrations were only a factor of three out for copper but did not explain the profiles of lead or cadmium. A similar analysis will be undertaken for the sediments of Hingham Bay to see if the identified species are important.

From Table 3.4 it is evident that if only inorganic ligands, such as sulfide and carbonate are considered, the anoxic zone of Hingham Bay is found to be saturated with respect to a 
pure $\mathrm{Ag}_{2} \mathrm{~S}$ phase, while both copper and lead are several orders of magnitude oversaturated with respect to CuS and $\mathrm{PbS}$.

In order to assess if the ligands measured by fuorescent derivatization can account for some of the apparent supersaturation of copper, equilibrium constants for the major porewater ligands are required. As not all of the major compounds have been identified yet, this is not possible. In addition, for the compounds that have been identified, such as 3-mercaptopropanoic acid, equilibrium binding constants are not readily available. However the role of polysulfides in solubilizing copper can be addressed, as the solubility of copper in the presence of covellite (CuS) and polysulfides has been studied in detail by Shea and Helz [1988]. The important reactions for dissolved copper speciation at the $\mathrm{pH}$ and total dissolved sulfide concentrations measured in the anoxic zone of Hingham Bay are given below. The equilibrium constants are converted to an ionic strength of $I=0.5$ using the Davies equation:

$$
\begin{array}{lr}
2 \mathrm{CuS}(\mathrm{s})+\mathrm{H}^{+}+3 \mathrm{~S}_{4}^{2-}+\mathrm{S}_{5}^{2-} \rightleftharpoons 2 \mathrm{CuS}_{4} \mathrm{~S}_{5}^{3-}+\mathrm{HS}^{-} & \log \mathrm{K}=6.5 \\
2 \mathrm{CuS}(\mathrm{s})+\mathrm{H}^{+}+\mathrm{S}_{4}^{2-}+3 \mathrm{~S}_{5}^{2-} \rightleftharpoons 2 \mathrm{Cu}\left(\mathrm{S}_{5}\right)_{2}^{3-}+\mathrm{HS}^{-} & \log \mathrm{K}=6.0 \\
\mathrm{Cu}^{2+}+\mathrm{S}_{5}^{2-}+\mathrm{HS}^{-} \rightleftharpoons \mathrm{CuS}\left(\mathrm{S}_{5}^{2-}\right)+\mathrm{H}^{+} & \operatorname{logK}=18.3
\end{array}
$$

It is unfortunate that the concentrations of polysulfides has not been determined accurately and that the species contributing to the two peaks could not be determined in this study. However as a preliminary assessment of the importance of polysulfides, it will be assumed that the dominant porewater polysulfides are total $\mathrm{S}_{5}^{2-}$ and $\mathrm{S}_{4}^{2-}[$ Luther et al., 1986], and that these species are peaks 8 and 9. Also assuming that the glutathione calibration is appropriate, each species can be estimated to range from 10 to $20 \mu \mathrm{M}$. From these assumptions the concentrations of the dissolved copper species in the anoxic zone can be calculated. The acid dissociation constants for these polysulfide species are $\mathrm{pK}_{a}\left(\mathrm{HS}_{4}^{-}\right)=6.7$ and $\mathrm{pK}_{a}\left(\mathrm{HS}_{5}^{-}\right)=6.1$. [Shea and Helz, 1988], so most of the compound is in the dissociated state at the $\mathrm{pH}$ found in porewaters. The free copper concentration is assumed to be in equilibrium with CuS, as discussed in Section 3.3.6. The calculated range in concentrations of each dissolved copper species is given in Table 4.2. The complex $\mathrm{CuS}\left(\mathrm{S}_{5}\right)^{2-}$ is the dominant complex, and because of its abundance the predicted aqueous dissolved copper concentrations are considerably closer to the measured average value of $17.9 \mathrm{nmol} / \mathrm{kg}$ than 
Table 4.2: Calculated concentration of copper-polysulfide complexes

\begin{tabular}{lc}
\hline Species & Concentration $(\mathrm{nmol} / \mathrm{kg})$ \\
\hline $\mathrm{CuS}_{4} \mathrm{~S}_{5}^{3-}$ & $0.3-1.2$ \\
$\mathrm{Cu}\left(\mathrm{S}_{5}\right)_{2}^{3-}$ & $0.5-1.9$ \\
$\mathrm{CuS}\left(\mathrm{S}_{5}\right)^{2-}$ & $8.4-16.8$ \\
Total $\mathrm{Cu}(\mathrm{aq})$ & $9.2-19.9$ \\
\hline
\end{tabular}

October 2003 conditions, $\mathrm{pH}=7.69, \mathrm{~S}_{\text {total }}=319 \mu \mathrm{M}$,

Total $\mathrm{S}_{4}^{2-}$ and $\mathrm{S}_{5}^{2-}$ taken to range from 10 to $20 \mu \mathrm{M}$

the estimates calculated by assuming only simple inorganic complexation, which predicted a concentration of $0.01 \mathrm{nmol} / \mathrm{kg}$ (see Table 3.4).

The great improvement between the measured copper concentration and the copper concentration calculated by including polysulfide concentrations is very encouraging. There are other peaks in the chromatogram that may also be important in solubilizing copper that have not been considered due to lack of information, so this speciation model is by no means complete. However this analysis with only approximate polysulfide concentrations does demonstrate that the reduced sulfur compounds detected by monobromobimane derivatization are important in enhancing the solubility of copper in porewaters. A more accurate determination of the polysulfide concentrations and determination of the stability complexes of copper with 3-mercaptopropanoic acid will enable a more complete speciation model to be undertaken. When stability constants for polysulfides and 3-MPA become available for lead, it will be very interesting to assess if there are similar improvements in the predicted porewater concentrations of lead.

\subsection{Future Work}

A considerable amount of work remains to be done on the compounds detected in this study. The most pressing issue is that of positively identifying the peaks that have been tentatively identified. Running the derivatized porewaters and spiked porewaters on a different chromatography column and verifying that co-elution still occurs is required. Once verified, quantifying the polysulfide peaks is a priority, as these compounds seem to be very important, both for solubilizing copper in the anoxic zone of the sediments and also as sediment derived strong ligands in the overlying water column. Synthesis of pure polysulfide 
phases by the methods of Kamyshny et al. [2003] will provide more robust solutions against which to calibrate.

There are a number of peaks that have not been identified, including one major peak and two minor peaks that indicate that they flux to the overlying waters. Dupont et al. [2004] demonstrated the power of electrospray ionization ion-trap mass spectrometry in identifying unknown thiol compounds, so it is likely that this would be a successful method to determine the identity of the unknown peaks. Mass spectrometry also seems to be a good way to determine the relative ratios of different polysulfide species, information that is important for metal speciation calculations.

The calculated flux of glutathione, 3-MPA and polysulfides suggests that the sediments could be an important source for these compounds in Boston Harbor. These compounds are strong ligands for copper and soft metals, so the sediments play a role in influencing the metal speciation in the waters of Boston Harbor. To verify that the gradient determined from porewater sampling gives an accurate estimate of the flux across the sediment-water interface, samples from benthic chambers should be derivatized and analyzed. The flux measured in the benthic chamber could then be compared with the porewater predicted flux. Waters from Boston Harbor should also be analyzed to see if compounds identified in the porewaters are found in the overlying waters. In order to accurately measure thiols in waters at $\mathrm{nM}$ concentrations, a pre-concentration technique, such as that developed by Tang et al. [2003] or Dupont et al. [2005], will be necessary.

Determining the oxidation rates of these reduced sulfur compounds in oxygenated waters is also important for determining if these compounds influence the speciation of metals in the waters of Boston Harbor. Polysulfides have been shown to oxidize slowly in the laboratory [Giggenbach, 1974]; however the rates of oxidation in the environment will be different and should be investigated. Investigating the roles trace metals play in modifying the oxidation rate of sediment-derived ligands would also be very interesting. Complexation may simultaneously protect the ligand and hold the metal in the dissolved phase, and so be important in determining the long-term fate of the both ligands and trace metals in the water column.

Preliminary speciation calculations on the porewater reduced sulfur and metal data suggest that polysulfides are very important for enhancing the dissolved concentrations of copper. Once the other compounds are identified and all species are well quantified, a more 
detailed speciation model for copper, lead and silver should be undertaken to determine more accurately the importance of reduced sulfur compounds in controlling metal concentrations in anoxic environments. Determination of equilibrium constants may be necessary due to the scarcity of thermodynamic data.

\subsection{Conclusions}

Boston Harbor porewater, when derivatized with monobromobimane, generates several fluorescent adducts that show variations with depth. The $\mathrm{pH}$ of the reaction is controlled to optimize for thiol derivatization and the relationship between each compound and the sulfide concentration indicates strongly that these compounds are thiols or other reduced sulfur compounds. Assignment of peaks based on retention times suggest that 3-mexcaptopropanoic acid is a major thiol in the porewaters and glutathione and mercaptosuccinic acid are minor components. Some additional work is needed to confirm these assignments. Two additional peaks are tentatively assigned to be polysulfides.

The thiols and polysulfides are produced in the sulfidic zone and reach concentrations of up to $10 \mu \mathrm{M}$ by $20 \mathrm{~cm}$ depth. The only exception to this is glutathione, which is found at a maximum concentrations in the sub-oxic zone, at concentrations of up to $0.13 \mu \mathrm{M}$. The very different profile of glutathione compared to the other compounds suggests that glutathione, unlike all the other compounds, is generated by a mechanism that is not dependent on the sulfide concentration. This likely reflects its biological source.

Thermodynamic calculations of the copper speciation in anoxic porewaters suggest that the polysulfide species found in the sediments are likely to be very important in controlling the solubility of copper. Initial thermodynamic calculations of the copper concentration in porewaters using only sulfide and other inorganic ligands resulted in underestimates of the actual concentration by three orders of magnitude. Inclusion of polysulfides in a model of speciation gave estimates that agreed with the measured concentrations within a factor of two.

Most of the measured thiols are removed from porewaters below the sediment-water interface. However, the profiles of at least five compounds indicate that they resist oxidation in the sub-oxic sediment and can diffuse across the sediment-water interface. Calculation of the porewater flux of glutathione, 3-MPA and polysulfides indicates that diffusive release 
from the sediments may be an important source of strong metal binding ligands to the waters of Boston Harbor. Further verification of this is required, however this preliminary evidence suggests that ligands generated in the sediments may play an important role in influencing the metal speciation in overlying waters. 


\title{
Chapter 5
}

\section{The Role of Sediment}

\section{Resuspension in the}

\section{Remobilization of Metals from}

\section{Coastal Sediments}

\begin{abstract}
Sediments are a major repository of contaminant metals in the coastal zone. Due to shallow water depths, the sediments in coastal areas are often subject to regular episodes of sediment resuspension. However, the impact of sediment resuspension on the release of metals is poorly understood. The aim of this Chapter is to address this issue and to quantify the release of metals and nutrients due to resuspension at two contrasting sites in coastal Massachusetts. The fluxes of metals and nutrients over the range of shear stress conditions encountered in the environment were measured with a laboratory-based erosion chamber. The fluxes measured in the erosion chamber were coupled with a record of shear stresses simulated by a hydrodynamic model in order to fully quantify the magnitude of release due to sediment resuspension. Sediment particles enriched in metals 4-50 times greater than the bulk surface sediment concentration were the first particles to be eroded. As particles with high concentrations of metals are eroded at shear stresses that occur at least monthly, the importance of sediment resuspension in mobilizing metals is enhanced. The quantity of copper and lead in the particulate phase that was mobilized by sediment resuspension was found to be greater than the combined (dissolved + solid phase) total of all other quantified loadings (sewage effluent, river discharge, stormwater and groundwater) to Boston Harbor. If the solid phase was maintained in suspension, $5-7 \%$ of the particulate copper and silver was released to the dissolved phase after 90 hours. The release of lead and iron to the dissolved phase was less than $1 \%$. Sediment resuspension also immediately released solutes to the water column. The concentrations of nutrients, silver, lead, copper and iron were
\end{abstract}


all greater than predicted based on conservative mixing of porewaters with the overlying water. Sediment resuspension thus enhances the release of material to the dissolved phase. The total benthic release of copper to the dissolved phase is $30 \%$ of the total quantified loadings to Boston Harbor and is $9 \%$ for lead. Sediment resuspension thus mobilizes significant quantities of metals from contaminated sediments and is responsible for transferring a fraction of this material from the solid phase to the more bioavailable and mobile dissolved phase. The importance of sediment resuspension as a source of metals to Boston Harbor is expected to increase as continued pollutant control decreases the inputs from other sources.

\subsection{Introduction}

The sediments of many coastal waters have become large repositories of contaminants. Particle-reactive species, such as metals and hydrophobic organic compounds, when discharged to the environment become associated with suspended solids. When they encounter regions of low energy within the water column, the particles settle to the seafloor, bearing with them their contaminant load. With decades to centuries of pollutant discharge, the sediment inventory of contaminants in some coastal areas has become significantly greater than crustal background levels [Ankley et al., 1996]. The sediments are not necessarily a permanent sink for contaminants, so that release from contaminated coastal sediments now represents a potentially important source of pollutant species to coastal waters [RiveraDuarte and Flegal, 1997a; Warnken et al., 2001].

The focus of this paper is on the remobilization of metals and nutrients from fine grained coastal sediments. Mechanisms driving the release of these species from sediments are molecular diffusion of porewater species across the interface, upward advective flows of porewaters and bulk mixing of sediment and porewater into the water column. Diffusive transport is a molecular level process driven by a concentration gradient across the sediment-water interface. Advective flows in fine grained sediments can be generated by benthic organisms irrigating their burrows. Bulk mixing can be due to biological activity, sediment resuspension processes, and sediment slumping or human activities such as dredging [Saulnier and Mucci, 2000]. The relative importance of each of these mechanisms is dependent on the diagenetic environment within the sediment column, the relative mobility of a species under different redox conditions, the activity of benthic organisms, and the hydrodynamic conditions in the overlying waters.

For a metal to be released by diffusive or advective mechanisms it must be in the dissolved phase. Due to high particle reactivity and tendency to form insoluble mineral 
phases in sediments, the largest proportion of metals reside in the solid phase. Bulk mixing of the sediments is thus the only mechanism that can potentially remobilize the largest pool of metals within the sediments over short timescales. The impact of a bulk mixing event comes from two effects. The first is that it can lead to the transport of resuspended metals away from their site of deposition. The second is that the change in chemical environment experienced by particles eroded into the axygenated water column can lead to release of dissolved metals directly to the water column.

The bulk mixing process that is the focus of this paper is sediment resuspension. Sediment suspension occurs once critical flow conditions within the water column have been exceeded, so that particles are eroded and maintained in suspension by the exchange of momentum from the fluid to the particles [Dyer, 1986]. The major factors driving sediment resuspension at a site are the hydrodynamic environment as well as the characteristics of the sediment material. In coastal environments, tidal currents, wind waves, storm events and wave-current interactions are the most important factors causing sediment resuspension [Sanford et al., 1991]. Sediment resuspension occurs when the shear stress at the sediment-water interface is sufficient to disrupt the cohesive forces within the sediments. The grain size, mineralogy, density, the physio-chemical bonding of particles and aggregates as well as the presence and activity of benthic organisms [Amos et al., 1992a] determine the sediment's cohesive strength and thus its ability to resist the shear stress imposed by the overlying fluid.

The importance of a sediment resuspension event on the release of metals to the water column is dependent upon the redox environment in the sediments from which the particles came. In sediments with a surfical oxic layer, dissolved trace metals are scavenged by iron oxyhydroxides and manganese oxides [Klinkhammer et al., 1982; Shaw et al., 1990]. The surface sediments are thus enriched in trace metals by diagenetic cycling [Sundby et al., 1986; Westerlund et al., 1986]. Sediment resuspension acts on the surface sediments, so mobilizes these enriched particles into the water column. The fate of these particles in the water column is not known, but it is likely to be an important factor relocating metals.

In organic-rich coastal marine sediments sulfate reduction is an important pathway for the remineralization of organic matter [Canfield, 1989]. Trace metals can be co-precipitated with, or adsorbed to authigenic sulfide mineral phases close to the sediment-water interface [Morse, 1994]. Sediment resuspension can mobilize these sulfide phases into the water 
column, where they can be oxidized, so releasing the associated trace metals [Morse, 1994; Simpson et al., 1998].

Sediment resuspension is additionally important as it provides a short circuit to the diagenetic processes that can release metals across the sediment-water interface. Trace metals released to the porewaters in the sub-oxic or anoxic zones within the sediment can migrate downwards and co-precipitate as a sulfide phase. Alternatively, if they move upwards they can be scavenged by iron and manganese oxide phases or they can diffuse across the sediment-water interface [Elderfield et al., 1981]. Sediment resuspension enhances metal release from the suboxic zones, not only due to the exposure to oxygen in bottom water, but also because dissolved species are released directly to the water column so that the oxidative trapping mechanism within the oxic sediment layer is bypassed. Metals buried as sulfides below the maximum seasonal excursion of the oxic zone will be permanently sequestered in the solid phase of the sediments unless bioturbation or resuspension can move the solid phase metals upwards.

The erodibility of a sediment is quantified by the critical erosion shear stress (or critical erosion velocity, $\left.\mathrm{u}^{*}{ }_{c}\right)$ and the rate of erosion $[$ Dyer, 1986]. Due to the wide variety of parameters that can influence the erodibility of cohesive sediments, it is difficult to predict. Studies investigating the erodibility of cohesive sediments have used flume experiments to measure the magnitude and nature of suspension with increasing increments of bottom stress. In-situ flume deployments (eg Amos et al. [1992b]; Ravens and Gschwend [1999]) and laboratory-based flumes [McNeil et al., 1986; Tsai and Lick, 1986; Thomsen and Gust, 2000] have been used to collect data on the critical erosion stress as a function of depth below the sediment-water interface and time. The fluxes of particles [Thomsen and Gust, 2000; Amos et al., 1992a], protists [Shimeta and Sisson, 1999] and the hydrophobic organic pollutants PAHs and PCBs [Latimer et al., 1999] have been measured in erosion flumes. The fluxes of organic carbon and autrients associated with sediment resuspension were investigated by Tengberg et al. [2003] with an in-situ erosion chamber that operated at two shear stress levels only.

The release of metals due to sediment resuspension has been investigated in a number of ways. Several investigators have used slurry or stirring experiments in which sediments are agitated with a single energy to simulate a large erosion or dredging event [Gerringa, 1990; Petersen et al., 1997; Saulnier and Mucci, 2000; Koschinsky et al., 2001]. All of the studies 
on sub-oxic sediments indicated that suspension in an oxic water column does lead to the release of dissolved species due to oxidation of particulates from reducing zones, mixing of porewaters and changes in the sediment:water ratio. The deep-sea study of Koschinsky et al. [2001] investigated oxic sediments and found that metals released from the porewater were quickly scavenged, so that the net effect of the sediment mobilization was a removal of metals from the dissolved phase. The drawback of slurry experiments, however, is that the importance of such releases on the geochemical budgets of metals in the coastal zone remains unquantified as bulk quantities of sediment are suspended and the experiments are not designed to represent expected flow conditions or to mimic the environment suspended species would actually encounter.

Erosion chamber and mesocosm experiments have been undertaken by a number of investigators in order to overcome some of the limitations of slurry experiments and to assess the variations in sediment flux with changes in bottom shear stress. Laima et al. [1998] investigated the changes in sediment redox conditions and the flux of iron and manganese due to resuspension from sandy sediments. They found large releases of dissolved manganese due to resuspension that varied with stirring rate. They did not convert the stirring velocities they imposed into environmentally relevant shear velocities, so that their measurements cannot be considered in the context of the shear stresses encountered in the environment.

A mesocosm experiment that closely simulated the environmental conditions within Chesapeake Bay was undertaken by Kim et al. [2004] to investigate the effect of resuspension on the cycling of mercury. Mesocosms with surface areas of $1 \mathrm{~m}^{2}$ mimicked the tidal cycle of shear expected in the Bay by simulation of resuspension with 4 hour on and 2 hour off cycles. The experiment was monitored for a month and both resuspension and no-resuspension treatments were conducted. The different treatments reported different behaviors and indicated that total mercury was associated with resuspended particles, but that the mercury was not released from the particles during resuspension. Methyl mercury production rate was found to be higher in the tanks with resuspension, and this was suggested to be due to the enhanced oxidation of sulfide phases, improving the bioavailability of mercury to methylating bacteria. In order to ensure uniformity between treatments, the sediments in the mesocosm were homogenized and fauna removed prior to the experiment. The sediments used in the study were considerably disturbed in order to get them into the mesocosms. Although the sediments were equilibrated for 2 weeks in oxygenated water, 
the erosional characteristics and the redox chemistry within the sediments were probably altered relative to undisturbed sediments. The mesocosm experiments thus yielded valuable information on the role sediment resuspension plays in the biogeochemical cycles of mercury, but cannot accurately be used to quantify the mercury fluxes due to resuspension under in-situ conditions.

Laboratory-based erosion chamber experiments on undisturbed sediments have been used by a few investigators in order to address the variation in metal flux from undisturbed sediments under a variety of flow conditions. Two studies have used the Particle Entrainment Simulator [Tsai and Lick, 1986] to impose two levels of shear stress on selected sediments. Calvo et al. [1991] found that zinc and iron in resuspended particles from Venice lagoon samples were enriched relative to the concentrations in the sediments. Cantwell et al. [2002] found metal release to the dissolved phase and also enrichments in the suspended phase relative to the bulk sediment concentration in two anoxic Narragansett Bay sediments resuspended for 12 hours. Cadmium, copper, nickel, lead, zinc, iron and manganese were investigated. The release to the dissolved phase was found to be more influenced by the shear stress level imposed than was the release to the solid phase. The solid phase concentrations were found to be site specific and controlled by the chemical and physical composition of the site rather than the imposed stress level.

The slurry experiments and erosion chamber investigations of metal behavior due to sediment resuspension performed to date have indicated that metals in both the dissolved and solid phases can be released to the water column. However, none of these investigations have assessed how the release varies over the range of shear stresses expected to occur in the natural environment, nor have the shear stresses that were imposed been related back to the in-situ conditions.

The aim of this chapter is to employ an erosion chamber to investigate metal release over the complete range of shear stresses that are expected in the coastal environment, from quiescent to storm conditions. A combination of field observations and hydrodynamic model output will be used to relate the shear stresses imposed in the erosion chamber to those encountered in the environment, so that the erosion chamber results will have a physical context and the flux of metal due to sediment resuspension can be fully quantified.

The longer term fate of resuspended material will also be investigated. Slurry experiments indicate that depending on the metal, there is both a long-term release from sus- 
pended material as well as removal from the dissolved phase due to particle scavenging [Saulnier and Mucci, 2000]. The slurry experiments as well as the 12-hour erosion chamber experiments of Cantwell et al. [2002] were conducted at high suspended solids concentrations that are not representative of conditions that would normally be encountered in the water column. Incubations of resuspended particles at lower suspended solids concentrations were undertaken in order to get a more realistic indication of the long-term fate of metals released by sediment resuspension.

The geochemical environment of the sediments has important controls on the outcome of a sediment resuspension event. Two contrasting coastal sites were selected for this study, one a reducing, sulfidic site and the second a site with no measurable porewater sulfide. Selection of these two sites enables the roles of sulfide and iron and manganese oxides to be investigated. This study is unique in that the erosion experiments were conducted in conjunction with a thorough investigation of the redox environment within the sediments. High resolution sediment sectioning for porewater and solid phase analysis provides information on the sedimentary geochemical environment and metal cycling that a single surface sample cannot provide. In addition, the porewater data allow the estimation of diffusional metal fluxes across the sediment-water interface. The relative importance of diffusional release and sediment resuspension can thus be compared and a far more complete budget of benthic metal fluxes developed.

\subsection{Materials and Methods}

\subsubsection{Erosion Chamber Selection and Design Modifications}

A modified EROMES laboratory erosion device developed by the German GKSS Research Group [Tolhurst et al., 2000; Andersen et al., 2002] was used to determine the sediment response over the range of expected shear stresses. This chamber was selected over other currently available erosion chambers, such as the disc stirred Microcosm [Thomsen and Gust, 2000) and the Particle Entrainment Simulator [Tsai and Lick, 1986] due to a number

of factors. The ideal erosion chamber would produce a homogeneous stress distribution across the entire sediment-water interface. In a small erosion chamber suitable for use in a laboratory, this is an unrealistic goal due to the higher proportion of wall boundary layer effects to sediment surface area. EROMES produced a $20 \%$ variation in stress distribution 
across the surface, while the Microcosm produced a $5 \%$ variation [Gust and Muller, 1997]. However published studies using the $10 \mathrm{~cm}$ diameter Microcosm have only operated up to a shear stress of $0.2 \mathrm{Nm}^{-2}$, which does not represent the complete range of shear stresses expected at the study sites. The $20-\mathrm{cm}$ diameter Microcosm has been calibrated up to a shear stress of $0.5 \mathrm{Nm}^{-2}$ |Thomsen and Gust, 2000]. As well as operating over a wider stress range, the additional advantage of EROMES is that it is designed with a 10-cm core barrel, and is the same size as the sediment cores collected for porewater and solid phase sampling. Thus, no modification of the coring equipment was required to collect sediment for erosion experiments.

The design for EROMES was provided by Dr Rolf Reithmüller of GKSS. A propeller mounted $3 \mathrm{~cm}$ above the sediment surface generates turbulence within the core barrel, and baffles suppress full body rotation of the water column. In order to create a chamber suitable for trace metal sampling, all the metal parts were replaced with plastic. This led to some design modifications. In particular, the baffle system, originally constructed as a removable stainless steel ring, was replaced with a fixed polycarbonate baffle system of the same dimensions. The stainless steel propeller was coated with teflon. The propeller was driven with an Orbital Motors (Braintree, MA) stepper motor with a basic step angle of $0.72^{\circ}$. A mounting system for the motor was designed and constructed to minimize vibrations and to ensure the propeller could be placed vertically and consistently within the erosion chamber. The mount for the motor was fixed to a solid polycarbonate cap that fit snugly inside the erosion core barrel. The modified EROMES chamber is shown in Figure 5-1. Water and suspended sediment samples were collected from the erosion chamber by sampling through a port drilled through the cap. Either a syringe or a peristaltic pump was used to remove samples.

\subsubsection{Erosion Chamber Calibration}

The imposed bottom shear stress is a function of the propeller rotation rate. Sieved sand was used to calibrate the chamber. The rotational rate of the propeller was incrementally increased until significant movement of sand grains was observed. The shear stress required to resuspend the known grain size was determined from a Shield's threshold curve [Madsen, 1975]. The results of the calibration (Figure 5-2) show a linear response between the rotation rate and shear stress at the sediment-water interface. To investigate readily erodible 


\section{Erosion Chamber}

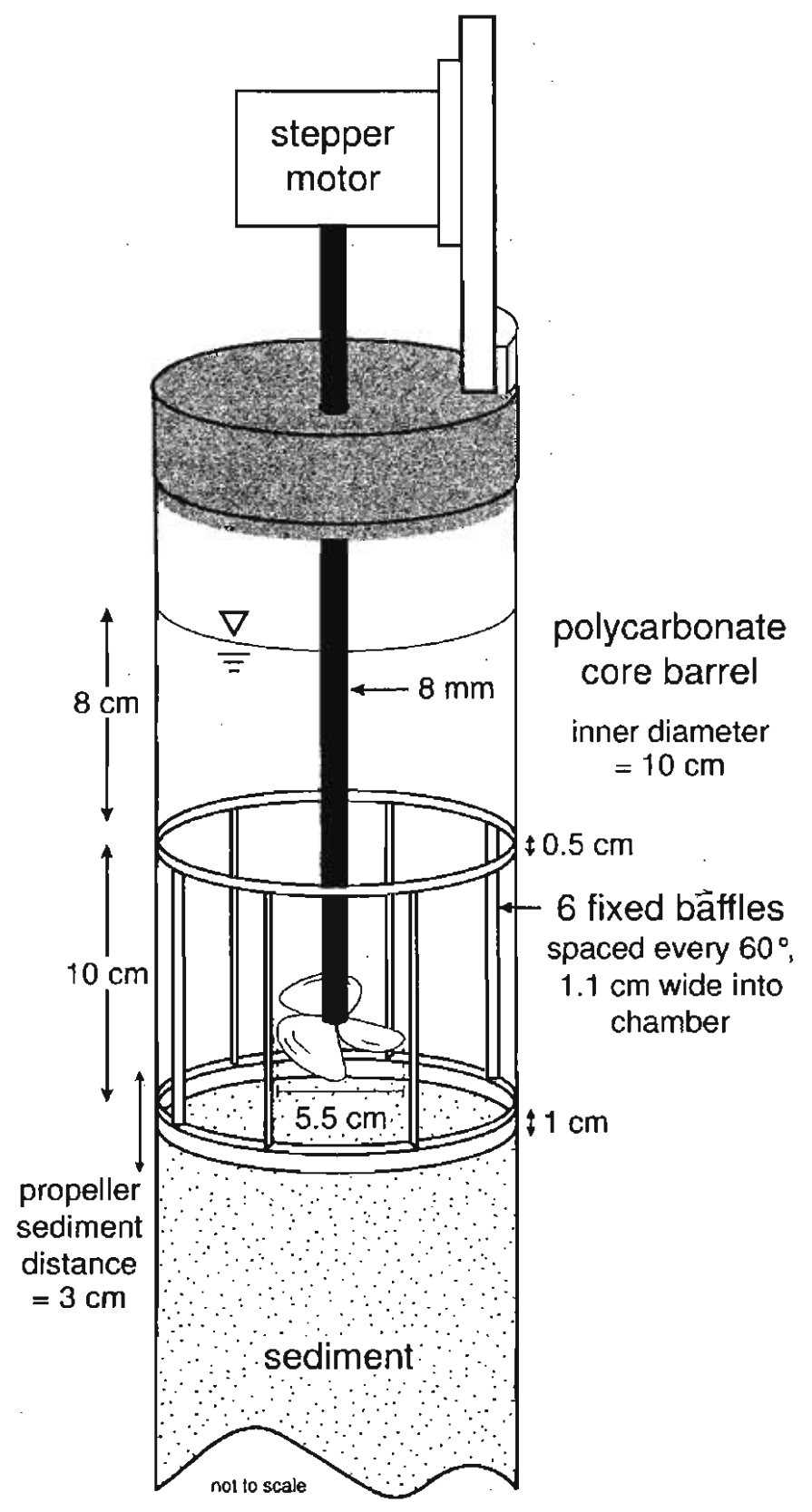

Figure 5-1: The modified EROMES erosion chamber showing the experimental geometry. Drawing by M. Sulanowska. 

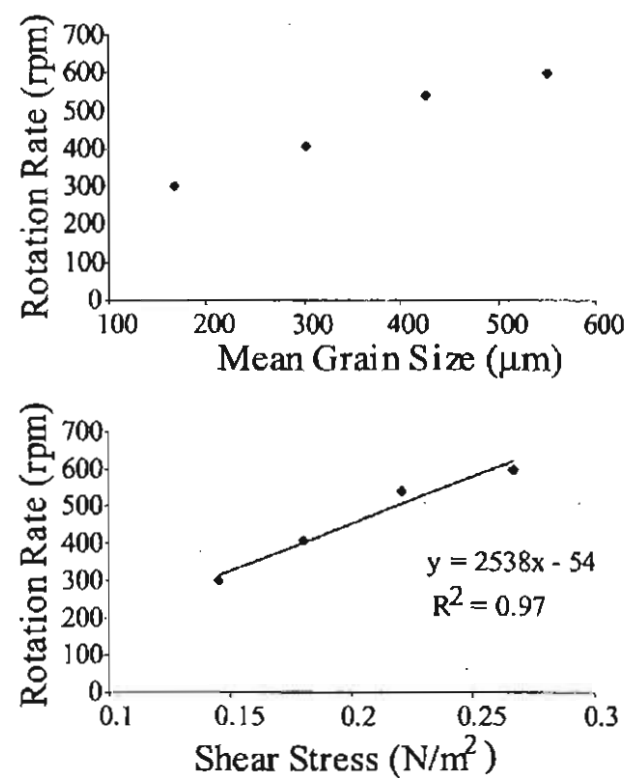

Figure 5-2: Erosion chamber calibration curves. (a) Rotation rate at which grains were first held in motion. (b) Shear stress calibration calculated from the Shield's curve for each grain size.

sediment, the calibration range would need to be extended to include lower shear stresses. This calibration range covers the erosion thresholds encountered at the two study sites but should be extended to increase the utility of the chamber. The calibration also needs to be extended on the high shear stress end as well, as some of the imposed shear stresses fall outside the range of the calibration. When this occurred an extrapolation of the calibration curve was used. Experiments with both larger and finer grain sizes than those already used will remedy this situation and will be undertaken in the near future.

\subsubsection{Field Sites}

Erosion studies were undertaken at two fine-grained sediment sites, one in Massachusetts Bay (MB) and the other in Boston Harbor (Figure 5-3 and Table 5.1). The first site, located in the north-western portion of Massachusetts Bay, is $9 \mathrm{~km}$ offshore in $30 \mathrm{~m}$ of water. The site is located $2 \mathrm{~km}$ west of the newly constructed sewage outfall for Boston. The site is in a region that is classified as depositional based on the presence of fine grained sediments [Knebel et al., 1991]. Surface waves generated by winter storms from the northeast are known to cause large sediment resuspension events [Signell and List, 1997; Bothner et al., 2002]. Since 1989, the United States Geological Survey has deployed current meters and 
Table 5.1: Site Characteristics and Locations

\begin{tabular}{|c|c|c|c|c|c|}
\hline Name & Location & Water Depth & Collection Method & $\%$ sand/silt/clay 1 & $\% \mathrm{C}_{\mathrm{org}}{ }^{\mathrm{T}}$ \\
\hline Massachusetts & $42.38981 \mathrm{~N}$ & $30 \mathrm{~m}$ & damped & $18 / 58 / 24$ & 2 \\
\hline Bay (MB) & $70.8305 \mathrm{~W}$ & & piston core & & \\
\hline Hingham & $42.29083 \mathrm{~N}$ & $5 \mathrm{~m}$ & diver & $6 / 62 / 32$ & 3 \\
\hline Bay $(\mathrm{HB})$ & $70.92778 \mathrm{~W}$ & & collected & & \\
\hline
\end{tabular}

${ }^{1}$ Data courtesy M. Bothner USGS for $0-0.5 \mathrm{~cm}$ depth range

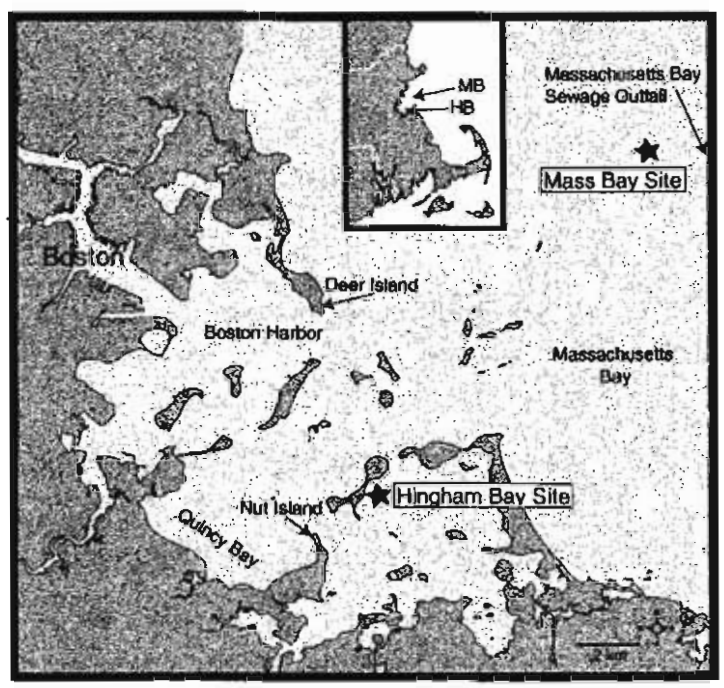

Figure 5-3: Map of coastal Massachusetts showing sampling site locations

sediment traps $4 \mathrm{~km}$ from the site. The current speeds are available at $5 \mathrm{~m}$ below the surface and at 1 and $10 \mathrm{~m}$ above the bottom. Wind and wave height data for the site are available from the National Oceanic and Atmospheric Administration meteorological buoy number 44013 also located $4 \mathrm{~km}$ away.

The Boston Harbor site is a much more sheltered site in the south-east corner of Boston Harbor, in Hingham Bay. The site is also in a depositional environment [Knebel et al., 1991] with an average water depth of $5 \mathrm{~m}$. Until 1991, solid sewage sludge was discharged from the nearby Nut Island Effluent outfall, so that the site is contaminated with high concentrations of metals and other contaminants [Bothner et al., 1998]. Unlike the MB site, no time series data of physical parameters is available. However, the sediment resuspension characteristics of nearby Quincy Harbor sediments have been determined [Ravens, 1997]. Weak tidal currents plus local wind waves generated during storms are the dominant hydrodynamic forcing in the area. 


\subsubsection{Sampling}

Sediment samples from both sites were collected in ways that minimized the disturbance of the sediment-water interface. Divers using SCUBA collected cores from the shallow Hingham Bay site, while at the Massachusetts Bay site a hydraulically damped piston corer [Bothner et al., 1998] was used. Core barrels were polycarbonate with a diameter of 10 $\mathrm{cm}$. Sediment collected in core barrels modified with fixed baffles, for use in the erosion chambers experiments, was obtained in exactly the same manner as when standard core barrels were used.

At each site, collected cores were placed on ice immediately after collection and brought back to shore. Cores for porewater and solid phase analysis were sectioned as soon as logistically possible. For Hingham Bay samples, the cores were driven back to WHOI and sectioned in a $4^{\circ} \mathrm{C}$ walk-in refrigerator, with processing complete within 8 hours of sample collection. Massachusetts Bay samples were sectioned at ambient air temperature in a van parked on the dock in Boston, so sectioning was complete within 6 hours of sample collection. More details on the sediment sectioning and sampling procedures are provided in Chapter 2.

The cores for erosion experiments were carefully transported to WHOI and refrigerated at $4^{\circ} \mathrm{C}$ until the experiments could be undertaken. For Hingham Bay samples, experiments were started within 6 hours of core collection. The Massachusetts Bay experiments were started early on the day after core collection, so that up to 18 hours had elapsed.

\subsubsection{Erosion Experiments}

The erosion core barrel was mounted rigidly in a stand, and the sediment-water interface was moved upwards with a hydraulic jack so that the surface was $1 \mathrm{~cm}$ below the bottom of the baffle. The experimental geometry was identical to that of the shear stress calibrations and is shown in Figure 5-1. An optical backscatter sensor (OBS) was mounted on the outside of the core barrel and the stirring propeller inserted into the core barrel. A control core was treated in the same manner but was not subject to any shear stresses, to assess if any non-erosional release of material could occur over the timespan of the experiment. All items used during the experiment for sampling and storage were cleaned with trace-metal clean protocols described in Chapter 2. 
A sample was withdrawn from the overlying water of both the erosion chamber and the control core before the propeller was started to represent the initial concentration conditions. The rotation rate of the propeller was then incrementally increased, until a maximum rotation rate of between 720 and $1000 \mathrm{rpm}$ was reached, corresponding to a final shear stress of $0.3-0.41 \mathrm{Nm}^{-2}$, depending on the experiment. Ten to twelve stress increments were imposed for each experiment. The core was not moved during the duration of the experiment. Each rotation rate was held for 20 minutes to ensure that the resuspension at each imposed shear stress had reached steady state. The time to reach steady-state was selected based on the work of others; both Ravens and Gschwend [1999] and Amos et al. [1992a] held each shear stress level for 10 minutes during in-situ sediment resuspension experiments in Quincy Harbor and the Bay of Fundy respectively.

After 20 minutes at each rotation rate, water samples were withdrawn. $50 \mathrm{mls}$ of sample was removed from the chamber and replaced with the same volume of bottom water from a carboy that was filled at the time of core collection. Five mls of sample was filtered through a Milli-Q-rinsed $0.45 \mu \mathrm{m}$ filter disc (Pall-Gelman) for nutrient analysis. The remaining sample was filtered through a $47 \mathrm{~mm}$ pre-weighed, acid-cleaned, polysulfone $0.45 \mu \mathrm{m}$ filter paper (Nucleopore) using a Savillex teflon filtering rig. The filtrate was further subsampled, with $20 \mathrm{mls}$ further filtered with an acid-cleaned polycarbonate $0.015 \mu \mathrm{m}$ filter (Nucleopore) to enable analysis of the colloidal fraction. The filtrates for metal analysis were acidified to pH 1 with trace metal grade $\mathrm{HCl}$ (Fisher Optima). All water samples were subsequently stored in the refrigerator until analysis. Each $0.45 \mu \mathrm{m}$ filter paper was transferred to a quartz petri dish and dried under an infra-red lamp. The quantity of material on each filter paper was determined gravimetrically.

The samples collected from the erosion chamber without any exchange of the overlying water represent the net amount of sediment resuspended. Redeposition of eroded sediment may have occurred, so that the total amount of sediment eroded is underestimated. To assess the importance of redeposition, following the 20 minute equilibration and sampling of the $\tau=0.18 \mathrm{Nm}^{-2}$ shear stress a peristaltic pump was used to exchange the overlying water with a volume of bottom water equal to 3 chamber volumes. This effectively removed most of the suspended sediment, thereby considerably reducing the depositional flux. The rotation rate was held constant for an additional 20 minutes and a second sample collected so that any additional material eroded at the imposed shear stress could be quantified. From visual 
observations of the suspended solids concentrations it was obvious that very little additional material was resuspended during the second 20 minute period. Thus the overlying water was not exchanged at each shear stress step, as the additional amount to be suspended was not significant compared to the amount initially resuspended. In general the overlying water was exchanged 2-3 times in an experiment, with most of the exchanges completed at high shear stresses, when the TSS concentrations and hence potential redeposition fluxes were highest.

All data presented is corrected for both the volume removed for sample collection and volume removed for exchange of overlying water. The concentrations presented for each shear stress level thus represent net release from the start of the experiment up to the given shear stress level.

At the conclusion of the Hingham Bay June 2004 experiment, a subcore of the erosion core was collected so that a post-erosion porewater profile could be measured. The subcore was quickly sectioned and the sediments centrifuged. The porewater was filtered with a $0.45 \mu \mathrm{m}$ filter, and the porewaters were analyzed for dissolved silicate.

\subsubsection{Incubation Experiments}

For Hingham Bay samples at two shear stress levels, additional overlying water was removed from the chamber, and incubation experiments were undertaken to address the longer term fate of the resuspended particles. The water from the chamber was diluted with a known volume of bottom water to generate samples for incubation that were more representative of the total suspended solids (TSS) concentrations found in Boston Harbor during non-storm conditions, that are less than $10 \mathrm{mg} \mathrm{L}^{-1}$ (MWRA weekly water quality monitoring data). The final TSS concentration in the incubations was between 100 and $200 \mathrm{mg} \mathrm{L}^{-1}$, depending on the specific incubation. How these concentrations compare to in-situ conditions during a storm and storm waning is unknown. Aliquots were removed for nutrient and trace metal analysis, then half of the sample was filtered with a $0.45 \mu \mathrm{m}$ filter to remove the particulate material. The filtered and unfiltered samples were then placed in $1 \mathrm{~L}$ acid clean HDPE bottles, loosely covered and stirred with a teflon stir bar, to ensure any particulate material was kept in suspension. The bottles were kept in a water bath and maintained at a constant temperature. The October incubations were maintained at $15^{\circ} \mathrm{C}$ while incubations in June were maintained at a room temperature of $24^{\circ} \mathrm{C}$, higher than the in-situ temperature of $19^{\circ} \mathrm{C}$ 
due to a water bath malfunction. Samples for nutrients and trace metals were withdrawn regularly over the course of four days. The samples collected from the incubating bottles were filtered with both 0.45 and $0.015 \mu \mathrm{m}$ membranes.

\subsubsection{Sample Analysis}

Acidified water samples were analyzed for iron and manganese with either a Hitachi graphite furnace atomic absorption spectrophotometer (GFAAS), quantified with a matrix matched 5 point external calibration, or a Finnigan Element 2 inductively coupled plasma-mass spectrometer (ICP-MS), operated in medium resolution with an indium internal standardization based on the method of Rodushkin and Ruth [1997]. When the ICP-MS was used to quantify iron and manganese, the multi-element capability of the ICP-MS was utilized and barium was also measured in low-resolution mode.

Trace metal analysis of silver, lead and copper in water samples was undertaken on 5 to $10 \mathrm{mls}$ of acidified sample. A cobalt-ammonium pyrrolidine dithiocarbamate (Co-APDC) co-precipitation method was used to pre-concentrate the trace metals from the seawater matrix. The porewaters from the sectioned sediments were also analyzed with the CoAPDC method. Isotope dilution ICP-MS was used to quantify the samples. Additional details on the Co-APDC method are provided in Chapter 2. The solid phases from the sectioned sediments were analyzed by the USGS with a total digestion following the USGS Method [Briggs and Meier, 1999].

The metal content of the particulate material on the filter paper was determined with a total sediment digest based on the same method used by USGS to determine the solid phase metal concentrations of the sectioned sediments. The total digest uses a series of acids to digest the sample with additions of concentrated hydrochloric acid, nitric acid, perchloric acid and hydrofluoric acid. After all residual perchloric fumes were driven off, the final digest was reduced to a $50 \mu \mathrm{L}$ concentrated nitric acid extract and diluted to $5 \%$ nitric acid with Milli-Q water. Unused filter paper and empty vials were also taken through the procedure to determine the trace metal concentrations associated with the filter paper and digestion procedures. The concentrations of iron, manganese, silver, lead and copper were quantified in the filter paper digests with ICP-MS analysis on samples diluted with a 0.5 ppb In internal standard solution. A $5 \%$ nitric acid matrix external calibration as well as standard additions of a multi-element standard (Alfa Aesar) to selected samples were used 
to quantify the sample concentrations.

\subsection{Results and Discussion}

\subsubsection{Erosional Characteristics}

Three erosion experiments were undertaken to provide a seasonal comparison (Hingham Bay in June and October) and an inter-site comparison (Massachusetts Bay in October). The sediment surface at both sites in fall was largely free from benthic colonization except for polychaete worm tubes of diameter of $2 \mathrm{~mm}$ and height up to $0.7 \mathrm{~cm}$. The density of these tubes was 3,000 and 10,000 tubes $\mathrm{m}^{-2}$ at the Massachusetts Bay and Hingham Bay site respectively. In Hingham Bay in June there was a red algal mat over the entire sediment surface, and no polychaete tubes were visible at the surface.

The erosional behavior of the three cores was similar. At the lowest shear stress applied to the sediment $\left(0.04 \mathrm{Nm}^{-2}\right)$, filaments sticking out above the sediment-water interface began to move in the current and the feeding tendrils of the polychaete worms, which were retracted before any shear was imposed, came out. There was no visual evidence of particles lifted into the water column until a shear stress level of $0.09 \mathrm{Nm}^{-2}$. Although the concentration of eroded material was very low at this stage, there was considerable movement of particles along the sediment surface. By a shear stress of $0.14 \mathrm{Nm}^{-2}$ most of the sediment surface was in motion and particles were regularly lifted into the water column. Particles up to $5 \mathrm{~mm}$ in size were ripped from the interface, including obvious portions of the algal mat in the June Hingham Bay experiment. Further increases in the shear stress led to an increasingly turbid water column, and by $0.2 \mathrm{Nm}^{-2}$ the sediment-water interface was obscured by the high TSS concentration.

The suspended solid concentration at each shear stress level was quantitated gravimetrically (Figure 5-4). Although there was visual evidence of some particle erosion at shear stresses of $0.08-0.09 \mathrm{Nm}^{-2}$, a significant concentration of particles was not detected until the following stress increments. Due to the stochastic nature of the turbulence imposed by the EROMES stirring mechanism [Gust and Muller, 1997], it is likely that occasional high energy eddies are generated and that these eddies may be responsible for resuspending material before bulk resuspension occurs. The shear stress at which erosion was obviously occurring across the entire sediment surface coincides with the first significant measure- 


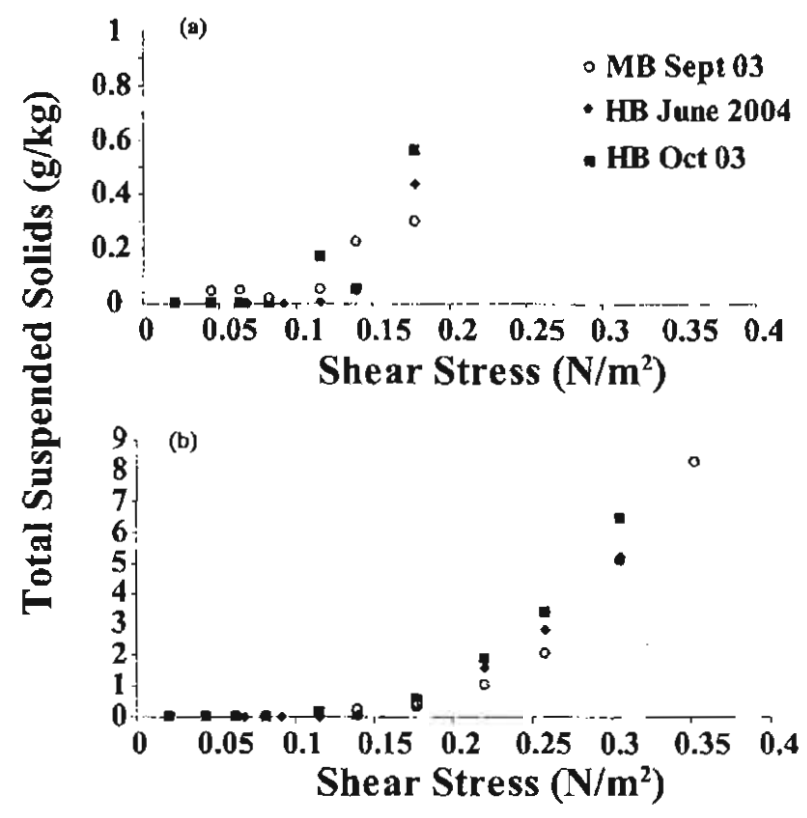

Figure 5-4: Concentration of total suspended solids in the erosion chamber at each shear stress increment. (a) the behavior at low shear stresses (b) enlarged scale to show response over the complete range of applied stresses.

ments of elevated TSS in the overlying water. This point thus represents the critical shear stress $\tau_{c}$, where the imposed shear stress first exceeds the cohesive forces due to intraparticle and gravitational forces within the sediments. The critical shear stress for both sites in the fall was $0.12 \mathrm{Nm}^{-2}$, and was slightly higher in Hingham Bay in late spring at $0.14 \mathrm{Nm}^{-2}$.

These values compare well to the erosion threshold determined by Ravens [1997] at three sites in nearby Quincy Harbor between August and October. In-situ linear flume experiments gave $\tau_{c}=0.10 \pm 0.04 \mathrm{Nm}^{-2}$ by optimizing the fit of erosion rate data to a linear erosion model. Ravens [1997] also found that there was a considerable decrease in erodibility when algal mats were present, and the obvious presence of an algal mat in late spring may be responsible for the higher erosion threshold reported here for June. In another study of Boston Harbor, Lee [1991] determined a critical shear stress range of 0.1 to $0.3 \mathrm{Nm}^{-2}$ using only tidal current data. The values are also in general agreement with other determinations at a range of nearby silty sites and with a variety of erosion devices. Shimeta et al. [2002] found a threshold shear stress of $0.25 \mathrm{Nm}^{-2}$ at a silty site in Buzzards Bay with the in-situ $2 \mathrm{~m}$ diameter annular flume Sea Carousel. Young [1975] reported a critical shear stress of $0.08 \mathrm{Nm}^{-2}$ in Buzzards Bay. In Baltimore Harbor Maa et al. [1998] 
deployed the VIMS Sea Carousel at four sites and reported a critical shear stress of 0.05 $\mathrm{Nm}^{-2}$ for the erosion of a fluff layer and then a critical bed shear stress of $0.1 \mathrm{Nm}^{-2}$ for the underlying silty-clay sediments.

\subsubsection{Erosion Depths}

Even at the highest shear stresses imposed in each experiment, the erosion depths did not exceed $5 \mathrm{~mm}$. The depth to which the sediment eroded at each shear stress increment was calculated from the porosity data for the surfical sediments (Table 5.2) and the measured TSS concentration. The sediments were assumed to have eroded uniformly over the entire core surface area. Due to topographical variation over the surface of the core (up to 0.5 $\mathrm{cm}$ in vertical variation across the core diameter), there may have been some preferential erosion in regions of elevated roughness. However, observations of the sediment surface at low shear stress levels could not discern any preferential erosion, the entire sediment surface appeared to be contributing particles to the water column. The porosity was not determined on the erosion core, as the experiment would have badly damaged the surface. Instead, porosity was determined on a core collected at the same time. The calculated erosion depths are shown in Figure 5-5. Resistivity measurements were undertaken 6 hours after the erosion experiments to obtain an additional estimate of the erosion depth however the data gave no indication of a distinct eroded layer. Resistivity measurements collected immediately upon completion of the erosion experiment may be more successful. At all shear levels, the Hingham Bay site was eroded to a deeper level than the MB site, and the Hingham Bay site eroded to essentially the same depth in each experiment. Although more material was eroded in $\mathrm{HB}$ in October, because the porosity in June was higher, the erosion depths are similar. These calculated erosion depths compare well with the work of Ravens (1997) who also found that at a shear stress of $0.5 \mathrm{Nm}^{-2}$ only $5 \mathrm{~mm}$ of sediment erodes from the Quincy Bay site.

\subsubsection{Solute Release}

The release of dissolved material during the erosion experiments is shown in Figure 5-6 to Figure 5-8. Only trace metals were measured in the first experiment conducted in Massachusetts Bay, while in the following Hingham Bay experiments, metals as well as nutrients were measured. The general trend for all species is an increase in concentration with in- 
Table 5.2: Porosity data for the surfical sediments at each site.

\begin{tabular}{lcc}
\hline & $\begin{array}{c}\text { Depth } \\
\text { (mm) }\end{array}$ & Porosity \\
\hline Massachusetts Bay & $0-2$ & $0.861^{a}$ \\
September 2003 & $2-4$ & 0.85 \\
& $4-6$ & 0.85 \\
& & \\
Hingham Bay & $0-2$ & 0.88 \\
October 2003 & $2-4$ & 0.84 \\
& $4-6$ & 0.82 \\
Hingham Bay & $0-2$ & 0.92 \\
June 2004 & $2-4$ & 0.92 \\
& $4-6$ & 0.90 \\
\hline
\end{tabular}

${ }^{a}$ Data for Massachusetts Bay October 2002

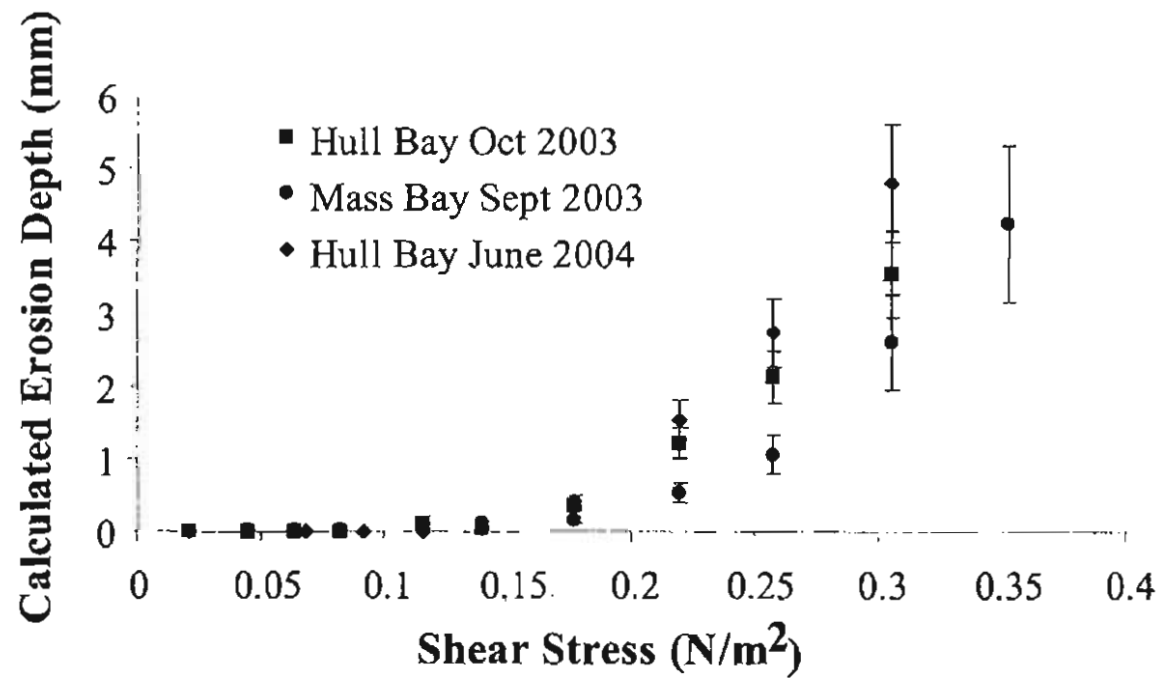

Figure 5-5: The calculated depth to which the sediment was eroded at each shear stress level. Error bars represent the standard deviation in calculated depth determined by error propagation of the standard deviation in porosity. 

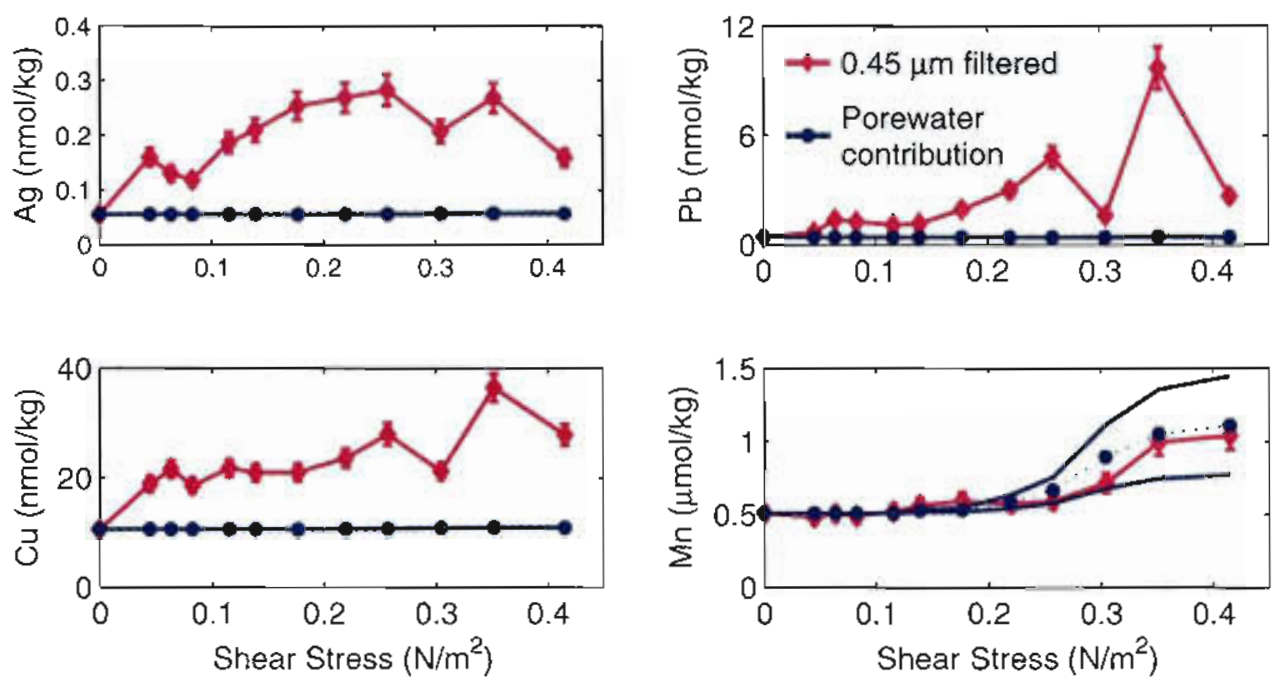

Figure 5-6: Dissolved phase concentrations at each shear stress level measured in Massachusetts Bay, September 2003. The red points are the measured concentrations in the chamber (corrected for overlying water removal) and the blue points are the concentrations expected based on porewater release from the eroded layer. The blue lines are the uncertainties in the porewater release estimate. Error bars are the standard deviation of the analysis.

creasing shear stress, but the details differ between species as well as between experiments.

The Massachusetts Bay experiment (Figure 5-6) showed a release of silver, lead and copper at the first erosion step, followed by no further release until the threshold shear stress of $0.12 \mathrm{Nm}^{-2}$ was reached. With further increases in shear stress the concentrations of the three metals continued to rise steadily until at $0.3 \mathrm{Nm}^{-2}$ there was a reduction in dissolved concentrations. At the next shear stress of $0.35 \mathrm{Nm}^{-2}$, the concentrations increased to the highest levels observed during the experiment. The concentrations fell again at the final shear stress of $0.41 \mathrm{Nm}^{-2}$. Manganese does not show the same trend as these metals, but instead shows little increase in concentration until a shear stress of 0.3 $\mathrm{Nm}^{-2}$, after which the manganese concentration rises slightly with subsequent increases in imposed shear.

The Hingham Bay experiment in October 2003 (Figure 5-7) differs from the MB results in that there is little or no observed release of dissolved species until after the threshold shear stress is exceeded. Then for all nutrient and metal species, except lead and copper, the concentration increases with each increase in shear stress. Ammonia, phosphate, silica, silver, iron and manganese all increase smoothly, while nitrate and copper show a sharp rise 

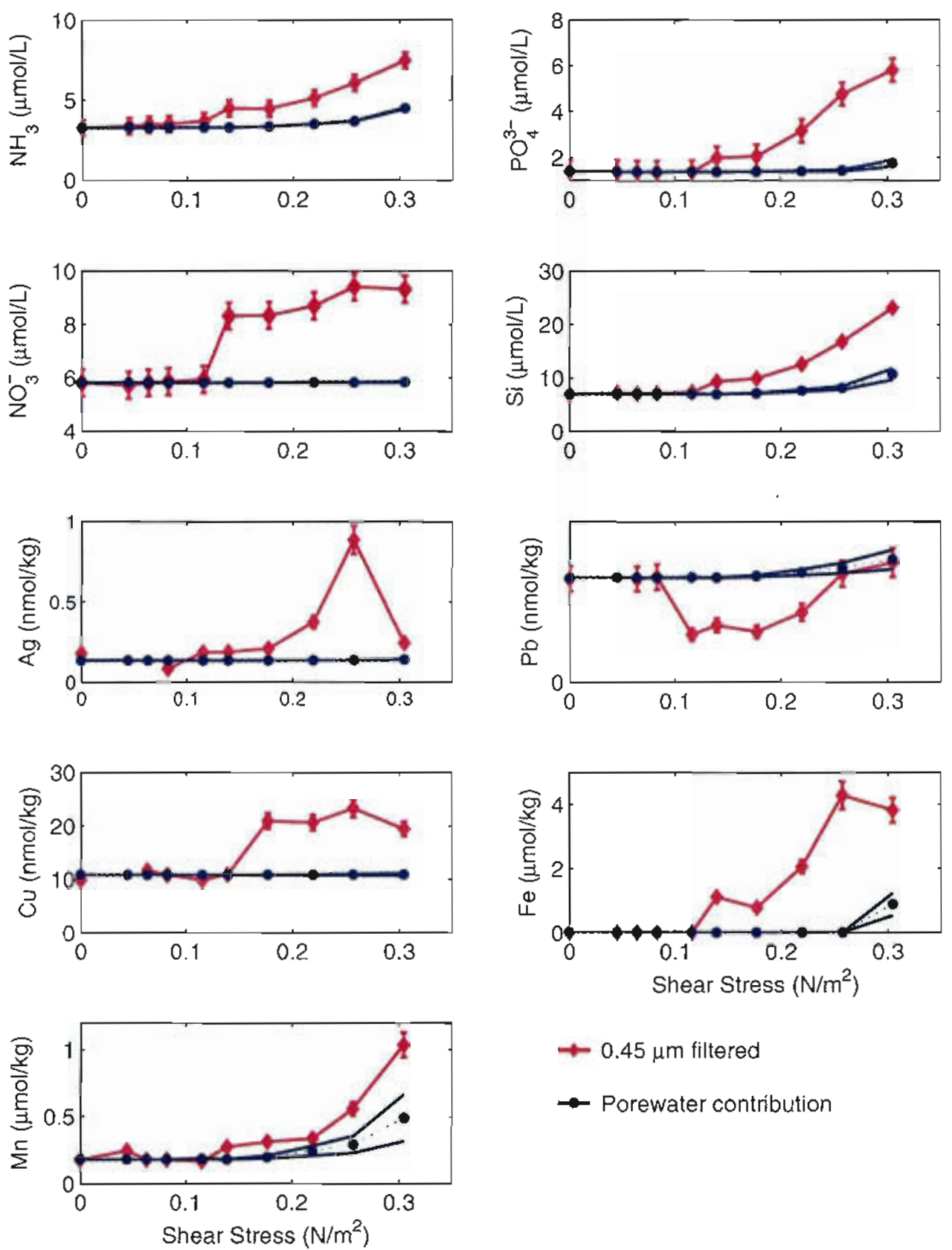

$0.45 \mu \mathrm{m}$ filtered

- Porewater contribution

Figure 5-7: Dissolved phase concentrations at each shear stress level measured in Hingham Bay, October 2003. The red points are the measured concentrations in the chamber (corrected for overlying water removal) and the blue points are the concentrations expected based on porewater release from the eroded layer. The blue lines are the uncertainties in the porewater release estimate. Error bars are the standard deviation of the analysis. 

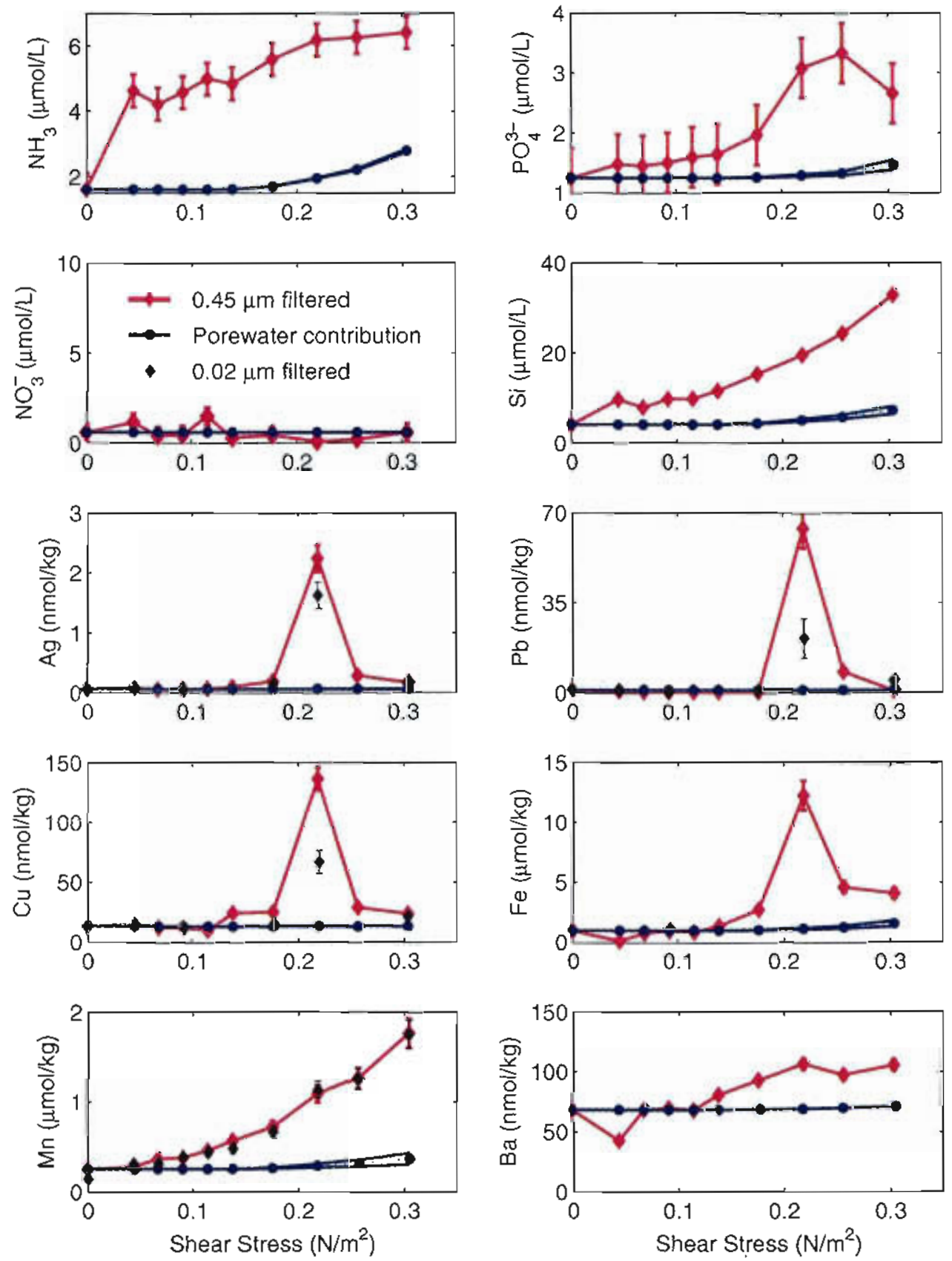

Figure 5-8: Dissolved and truly dissolved phase concentrations at each shear stress level measured in Hingham Bay, June 2004. The red points are the measured dissolved concentrations in the chamber (corrected for overlying water removal) and the blue points are the concentrations expected based on porewater release from the eroded layer. The blue lines are the uncertainties in the porewater release estimate. Error bars are the standard deviation of the analysis. 
once the threshold is exceeded. The concentration of nitrate continues to rise at a slower rate while that of copper stays constant. At the final shear stress level of $0.3 \mathrm{Nm}^{-2}$, the concentration of both iron and silver drops from the level measured in the previous shear step. Lead shows the opposite behavior, with a sharp decrease in lead concentration once the threshold stress is exceeded, a steadying of the concentration until a shear stress of 0.22 $\mathrm{Nm}^{-2}$ and then an increase, back to the initial concentration.

The release of nutrients in June 2004, (Figure 5-8), is similar to that observed in October (Figure 5-7) apart from the rapid release of ammonia, silica and phosphate, at the first shear stress level. The patterns of release measured in the successive shear stress steps are the same as observed previously. The concentration of silica is higher in June 2004 and the concentration of phosphate is the same until a shear stress level of $0.22 \mathrm{Nm}^{-2}$, after which the phosphate release in June ceases. No nitrate release is detected in June. The manganese release is also similar between experiments, except that there is some manganese release at $0.07 \mathrm{Nm}^{-2}$ in June, prior to the threshold shear stress, and the total manganese release in June is higher.

The release of the other metals to the erosion chamber in Hingham Bay in June 2004 is similar to that observed in October up to shear stresses of $0.2 \mathrm{Nm}^{-2}$. Then at a shear stress of $0.22 \mathrm{Nm}^{-2}$ there is a very strong release of iron, silver, lead and copper, but not of manganese or barium. The elevated concentration does not persist in the water column as by the next shear stress the dissolved concentration had fallen significantly. The concentrations of silver, copper and iron at the final stress level are essentially the same in both June and October.

The total amount of material measured in the dissolved phase due to the application of a shear stress is the net result of many competing processes. The changing concentration of dissolved species in the waters of the erosion chamber are due to porewater flushing as well as chemical reactions driven by the rapid change in environment experienced by a resuspended particle. Transport from the sediments to oxygenated waters can lead to oxidative release of reduced sediment species, such as metal sulfides [Simpson et al., 1998]. The reduced solute concentrations encountered by a particle lifted into the water column can lead to desorption of charged species adsorbed to solid surfaces [Morin and Morse, 1999] or the dissolution of precipitated mineral phases. Dissolved species released into the waters of the chamber do not necessarily persist in the dissolved state, but can be adsorbed 
onto resuspended solid surfaces or oxidized. The concentrations measured in the erosion chamber overlying water are thus the result of many varied and competing processes. The discussion below is an attempt to determine which of these processes are the most important in determining the magnitude of the dissolved flux.

\section{Mechanisms of Solute Release}

Resuspension lifts porewaters associated with the sediments into the overlying waters of the erosion chamber. The porewater concentrations measured in cores collected at the same time as the erosion core are shown in Table 5.3. The calculated concentration increase due to the release of porewaters from only the resuspended layer is also shown on Figures 5-6 to 58. The error bounds for the porewater release shown in Figure 5-6 to 5-8 were determined by error propagation of the standard deviation of the concentration and porosity in the sectioned cores.

For all species except lead and manganese, the concentrations exceed the contribution from the porewater associated with eroded sediments. Thus there is either non-conservative behavior associated with sediment resuspension, or the flow in the erosion chamber drives advection through the sediments, flushing porewaters from below the depth of resuspension. Laima et al. [1998] found the concentration of manganese in surface porewaters was reduced after a microcosm resuspension experiment, and attributed the measured rise in $\mathrm{Mn}$ concentrations in the erosion chamber to porewater release. However the sediments investigated in their study were sandy, and were thus more susceptible to advective flow then the fine-grained, cohesive sediments from the Hingham Bay or Massachusetts Bay sites.

To assess if porewaters were flushed from deeper within the sediment profile during the resuspension experiment, a porewater profile was collected from the erosion core after the June 2004 experiment. The comparison of the erosion core porewater profile and the two standard cores is shown in Figure 5-9. The concentration could not be measured on the core prior to the experiment so it is necessary to compare cores collected within 0.6 $m$ of each other. The concentrations in the shallow porewaters are considerably higher than those measured in the undisturbed cores. The uppermost porewater sample from the post-erosion core is 7.5 standard deviations away from concentrations measured in the undisturbed cores and higher than any dissolved silica value measured in the surface porewaters of the Hingham Bay site. The concentration in the erosion core is thus elevated 
Table 5.3: Porewater Concentrations in Surface Porewaters for Each Erosion Experiment

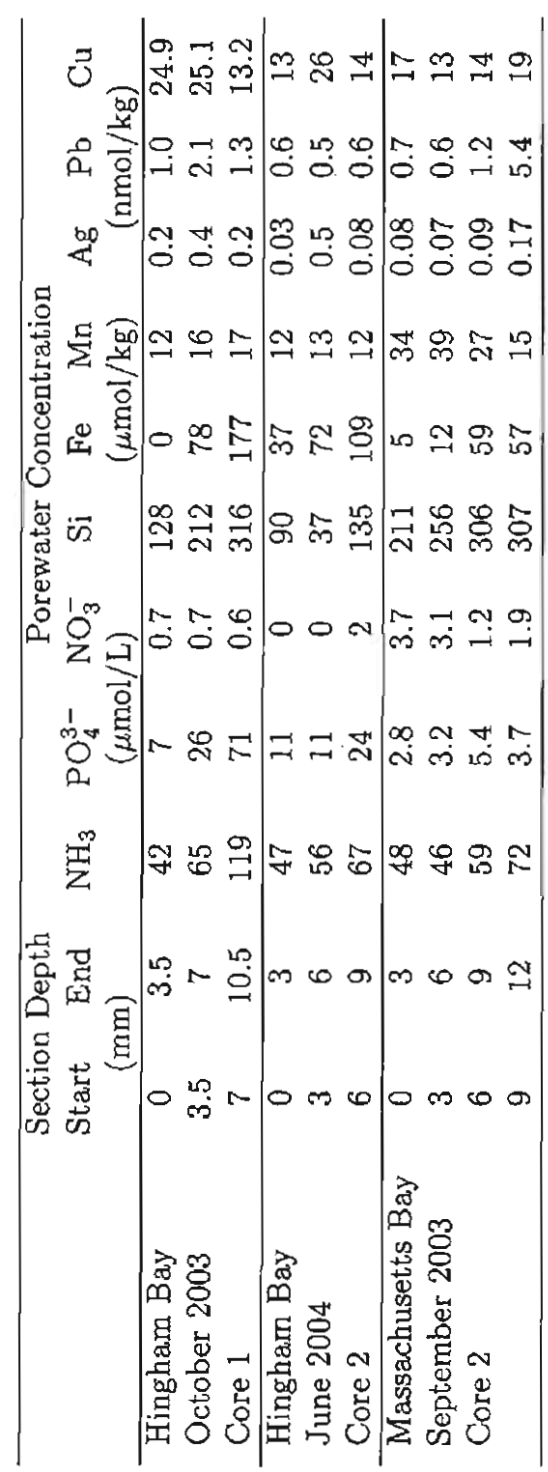




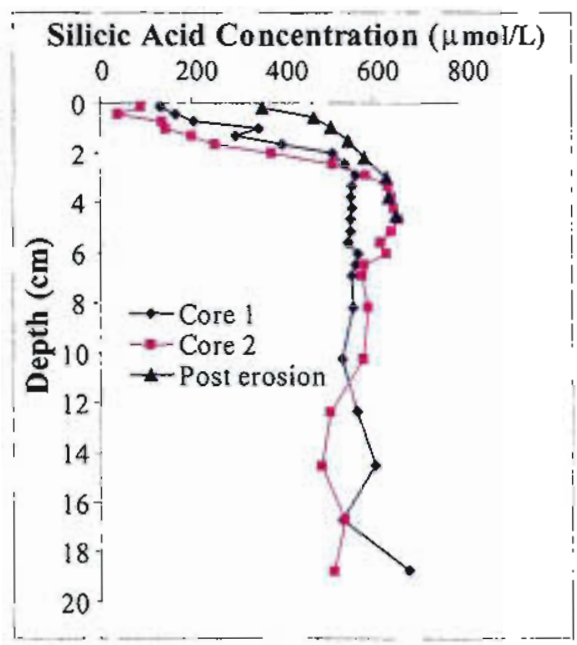

Figure 5-9: Hingham Bay porewater profiles of silicic acid from June 2004 and the profile from the core sectioned after the erosion experiment.

at the surface. The second porewater sample is still higher than the comparable data in the other cores, but the offset is not as great. By $1.5 \mathrm{~cm}$ the profiles reach similar concentrations. This suggests that resuspension has disturbed the uppermost sediments, but rather than a flushing occurring that lowers the concentrations in the porewaters by bringing in overlying water, the concentrations have actually risen. Even considering that the posterosion core profile should be offset downwards by $4 \mathrm{~mm}$ due to the loss of eroded material the concentrations still exceed those in the undisturbed cores. This very interesting result may be due to more concentrated porewaters from deeper within the sediment profile being brought towards the interface by an induced advection or due to the core slightly warming up over the duration of experiment. If the temperature was a significant contributor to the release of dissolved silica, it would be expected to increase the concentrations throughout the depth of the core and not just close to the surface. The erosion core concentrations below 2.5 $\mathrm{cm}$ are similar to those measured in Core 2, but are higher than the values found in Core 1. The roles of spatial beterogeneity and temperature are thus unclear. Further investigation of the mechanism behind this observed increase is warranted as these preliminary profiles suggest that following a resuspension, in addition to direct release due to resuspension there could also be an enhanced diffusive release of material due to elevated levels in the surface porewaters. Analysis of other porewater species such as ammonium and phosphate, as well as tracer experiments to determine the flow paths and depths of advective penetration into 
the sediments would be very helpful in resolving the important processes.

\section{Ion-exchange Reactions}

The non-conservative release of material to the erosion chamber may be due to ion-exchange reactions. The charged solute species in the porewaters can adsorb to the abundant solid surfaces in the sediment. Clays, organic matter and iron and manganese oxides are all potentially important adsorbents for ions in porewater. Particles in equilibrium with high solute concentrations in the porewaters are transported into dilute overlying waters, so that sediment resuspension can drive desorption of the adsorbed species. The porewater concentrations for the nutrient species given in Table 5.3 are all greater than the measured concentrations in the erosion chamber, so that the conditions required for ion-exchange are met in the overlying waters of the erosion chamber.

The ion exchange capacity of sediments with respect to the nutrient species ammonium [Morin and Morse, 1999] and phosphate has been studied in some detail [Krom and Berner, 1980]. It is thus useful to compare the extent of ion-exchange reported by these authors with the erosion chamber data to evaluate if an ion-exchange mechanism can account for the observed non-conservative release due to erosion. Both of these studies modeled the adsorption as a linear isotherm described by an adsorption co-efficient $\mathrm{K}^{*}$.

$$
K^{*}=\frac{C_{s}(e x c h)}{C_{p w}}
$$

Where $\mathrm{C}_{s}($ exch) is the concentration of exchangeable species in the sediment (mol/g dry weight) and $\mathrm{C}_{p w}$ is the measured porewater concentration (mol/L). The non-dimensional adsorption coefficient $\mathrm{K}$ is calculated from the porosity $\phi$ and the sediment density $(2.65$ $\mathrm{g} / \mathrm{ml})$ by:

$$
K=\frac{(1-\phi)}{\phi} \rho_{s} K^{*}
$$

From the published values for $\mathrm{K}$ and the measured porewater concentration, the amount of exchangeable material can be calculated. The concentration of either ammonium or phosphate expected in the erosion chamber $\mathrm{C}_{\text {chamber }}$, due to ion exchange from resuspended sediments, can thus be determined by

$$
C_{\text {chamber }}=K \frac{\phi}{1-\phi} \frac{1}{\rho_{s}} C_{p w} \frac{M_{\text {eraded }}}{V_{\text {chamber }}}+C_{0}+C_{r p w}
$$


Table 5.4: Literature values for the adsorption coefficient $\mathrm{K}$ and the concentrations expected in the erosion chamber due to ion-exchange reactions for Hingham Bay June 2004.

\begin{tabular}{lcc}
\hline & Ammonium & Phosphate \\
$\mathrm{K}$ & $0.7-7^{1}$ & $1.3-1.9^{2}, 34^{3}$ \\
$C_{0}(\mu \mathrm{mol} / \mathrm{L})$ & 1.6 & 1.3 \\
Calculated conc. in chamber due to ion-exchange $(\mu \mathrm{mol} / \mathrm{L})$ & $2.4-10.3$ & $1.4-6.0$ \\
$C_{r p w}(\mu \mathrm{mol} / \mathrm{L})$ & $1.1-1.2$ & $0.1-0.3$ \\
Conc. observed at $\tau=0.3 \mathrm{~N} / \mathrm{m}^{2}(\mu \mathrm{mol} / \mathrm{L})$ & 6.4 & 2.7 \\
\hline & & \\
& & \\
2 & & \\
$2[$ Morin and Morse, 1999$]$ coastal sediments with organic carbon contents ranging from $0.4-4.5 \%$ \\
$3[$ Krom and Berner, 1980$]$ oxic sediments, 1980$]$ anoxic sediments
\end{tabular}

where $M_{\text {eroded }}$ is the mass of sediment eroded into the chamber and $V_{\text {chamber }}$ is the chamber volume $(1.7 \mathrm{~L}), \mathrm{C}_{0}$ is the initial concentration in the erosion chamber and $\mathrm{C}_{\text {rpw }}$ is the contribution from eroded porewaters. The literature values of $\mathrm{K}$, the concentrations calculated from Equation 5.3 and the actual measured concentrations are shown in Table 5.4. For both ammonium and phosphate the measured concentrations fall within the range expected. This indicates that the elevated concentrations of ammonium and phosphate observed can be accounted for by release due to ion-exchange.

\section{Comparison of Ratios of Eroded Material with those in Porewater}

To determine if the solutes released to the overlying water are a similar composition to the porewaters, the N:P and Si:P ratio in the dissolved phase of the erosion chamber were determined and are shown in Figure 5-10 for Hingham Bay October 2003 and Figure 511 for Hingham Bay June 2004. The results of the linear regressions are summarized in Table 5.5 together with the ratios found in the porewaters of the upper 6 samples of the cores collected and sectioned at the same time as the erosion experiment was performed. The October and the June data both show strong linear relationships between the amount of phosphate released and the amount of ammonium, (ammonium+nitrate+nitrite) and silicic acid. In the June experiment there was a release of nutrients prior to the erosion threshold and a linear relationship between these points is also shown in Figure 5-11 that is different from the relationship observed once erosion is active.

In each experiment the Si:P ratio in the erosion chamber is higher than observed in the porewaters collected at the same time. This is additional evidence that there is a release 

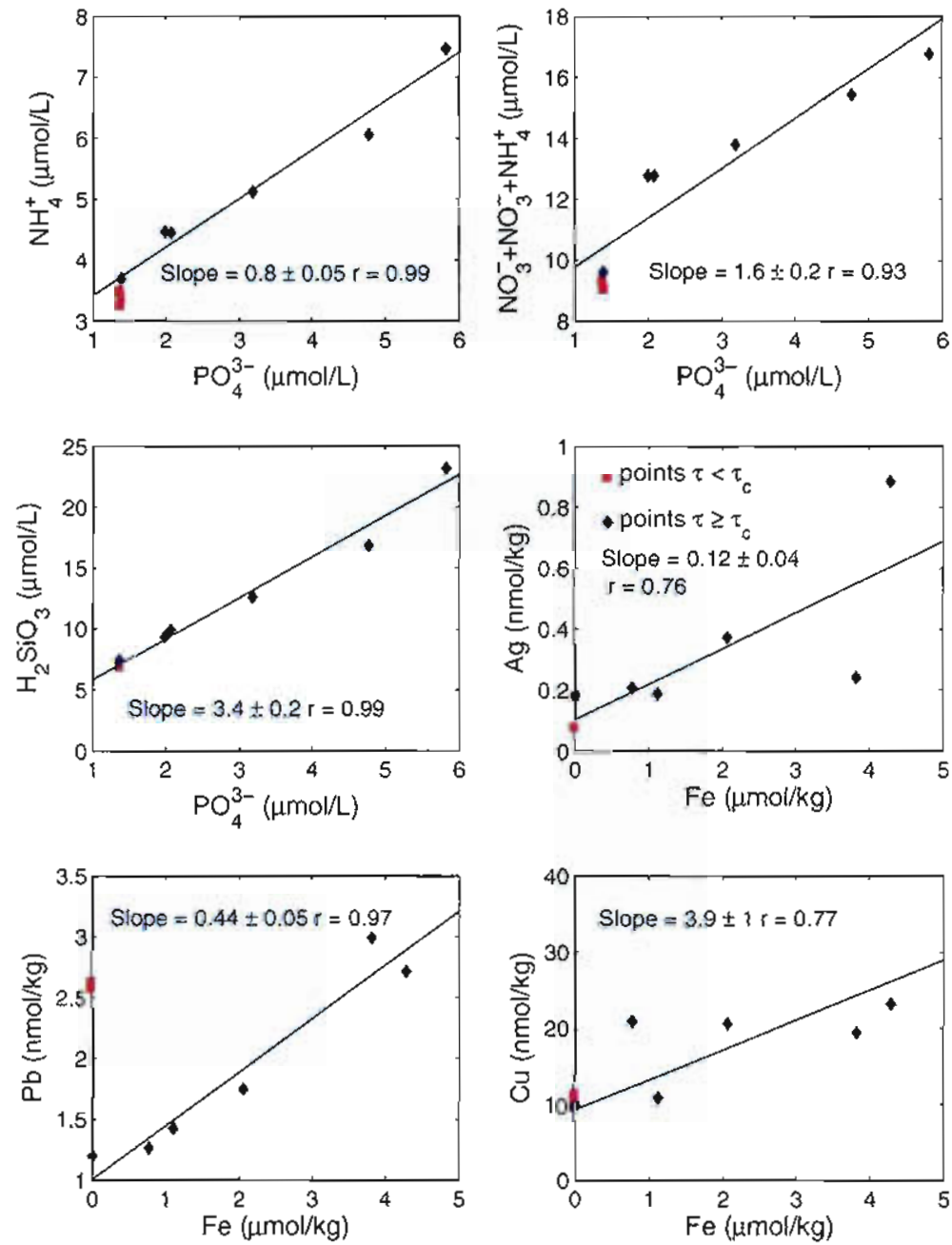

Figure 5-10: Linear regressions of dissolved concentrations measured in the erosion chamber at each shear stress step. Nutrient species are compared to phosphate and metals with iron for Hingham Bay October 2003 data. Legend shown in the silver panel applies to all species. 

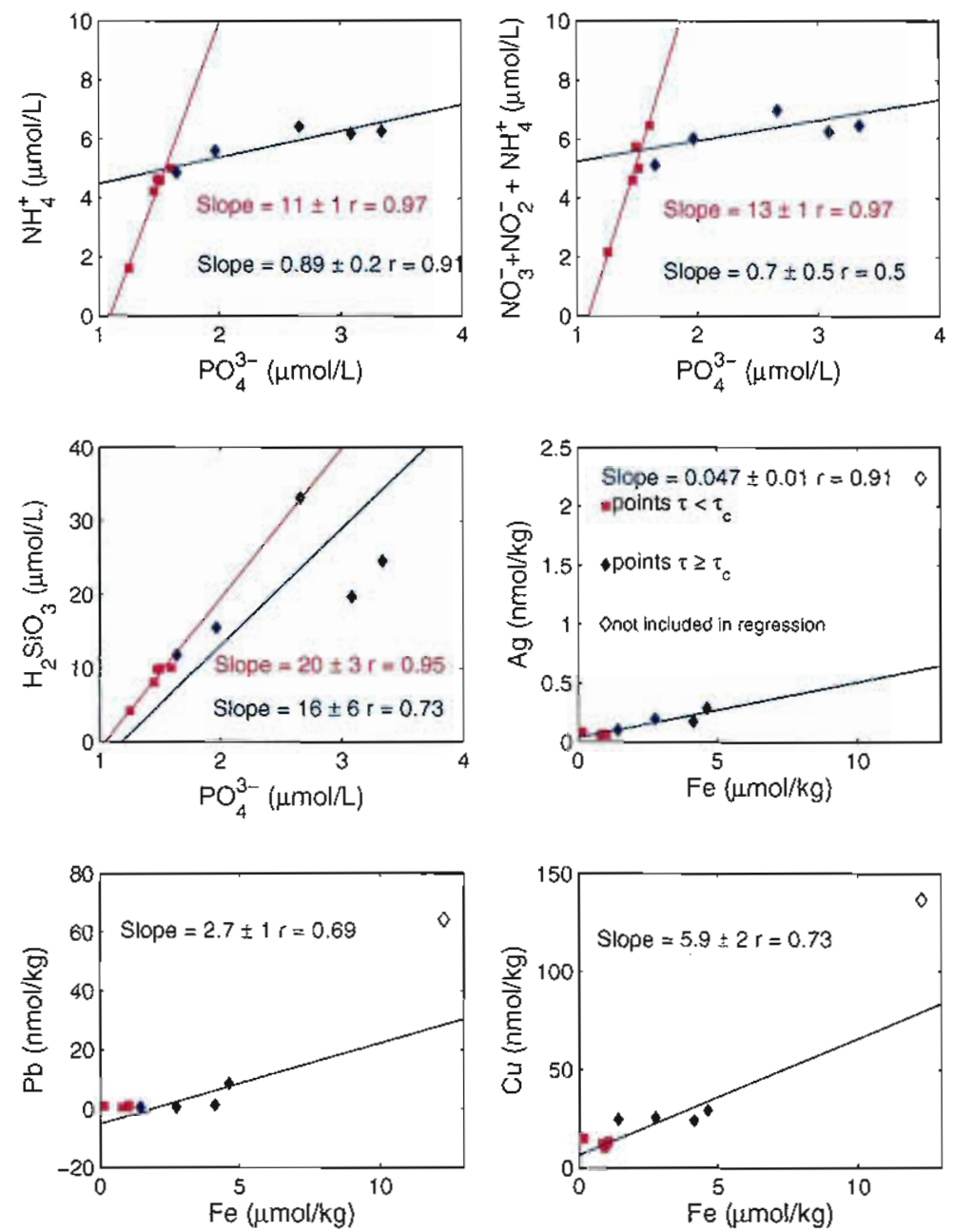

Figure 5-1.1: Linear regressions of dissolved concentrations measured in the erosion chamber at each shear stress step. Nutrient species are compared to phosphate and metals with iron for Hingham Bay June 2004 data. For the nutrient data two data populations are evident, the points below the erosion threshold and those after. Regressions have been done on each data population. 
Table 5.5: Comparison of the ratios in the erosion chamber dissolved phase with those in surface porewaters

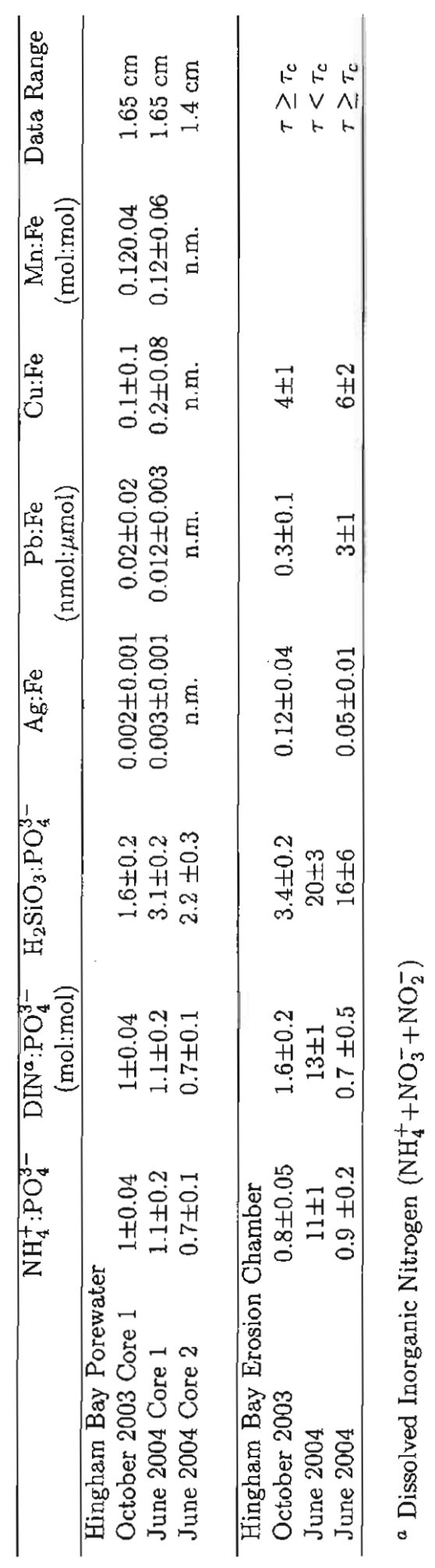


in excess of porewater flushing to the erosion chamber. Even if the dissolved species were due to flushing of porewaters from deeper within the sediment profile, the Si:P ratio would still be the same as observed in the average porewater. The Si:P ratio decreases with depth below the surface, so erosion of porewaters even deeper than $1.6 \mathrm{~cm}$ would not explain the much higher ratios. As this is not the case, some additional mechanism stimulated by resuspension has occurred that has led to more release of Si species relative to phosphate to the porewaters.

The nitrogen species do not show such distinctive behavior. As ammonium released into the water column can be oxidized to nitrate, the sum of (ammonium + nitrate + nitrite) is considered to take this into account. In October, the total inorganic nitrogen released to the erosion chamber had a slightly higher N:P ratio than found in the porewaters, while in June the ratios were comparable to those found in the porewaters. This suggests that if there is enhanced release of these species due to erosion, the process affects both total inorganic nitrogen and phosphate to similar extents.

The release of nutrients to the erosion chamber before bulk sediment was eroded is very interesting. With the first application of a shear stress, nutrients were released and continued to be released over the low shear stress range at a $N: P$ ratio of $13 \pm 1$, considerably higher than observed once erosion conmenced. The very different ratio may suggest that the most readily released nutrients are derived from a different pool of material, possibly from the degradation of fresh organic matter or part of the algal mat observed to cover the surface in June.

Table 5.5 also shows the concentrations of silver, lead and copper released to the dissolved phase ratioed to the concentration of dissolved iron. There is more variance in the metal relationships than with the nutrients. There is not a significant linear relationship between the concentrations of these metals and iron in the surface (upper $1.6 \mathrm{~cm}$ ) porewaters due to changes in the interactions between these metals in the different geochemical zones close to the sediment-water interface. However the average metal: iron (Me:Fe) ratio can be calculated for the surface porewaters, as given in Table 5.5. For both October and June the average Me:Fe ratio found in the porewaters is lower by at least one order of magnitude than the ratio observed in the erosion chamber. This again indicates that resuspension has led to non-conservative release of dissolved metals. 


\section{Scavenging}

In addition to processes adding solutes to the water column of the erosion chamber, removal processes are also active. Scavenging of dissolved species by particulates is an important process that strongly influences many trace metal distributions in the oceans [Donat and Bruland, 1995]. The importance of scavenging in the erosion chamber is highlighted by the lead data from October 2003 (Figure 5-7), the only species in which the concentration decreases with the onset of erosion. The concentration of lead in this experiment started at $2.59 \pm 0.03 \mathrm{nmol} / \mathrm{kg}$, which is elevated compared to bottom water, indicating that some lead contamination of the overlying water had occurred. As soon as the threshold shear stress was exceeded and there were particles in the water, the lead concentration dropped significantly. The timescale for the scavenging is obviously very fast, as only twenty minutes elapsed between each concentration point collected. Silver also shows a rapid drop in concentration in the October 2003 experiment, at a shear stress of $0.3 \mathrm{Nm}^{-2}$. Rapid scavenging is also evident in Hingham Bay in June 2004. At a shear stress of $0.22 \mathrm{Nm}^{-2}$ (Figure 5-8), a large pulse of iron, silver, lead and copper is released to the erosion chamber and by the next shear stress the concentrations have returned to values close to the concentrations observed before the pulse.

Similarly rapid scavenging was reported by Hatje et al. [2003] for cadmium and zinc adsorption onto suspended estuarine particles. Using radioisotopes of these elements they found that within 15 minutes $75 \%$ of the originally spiked metals had been removed from solution. The kinetics of copper uptake by particles in San Francisco Bay was investigated by Gee and Bruland [2002]. At ambient Bay particulate concentrations of $35 \mathrm{mg} / \mathrm{L} 15 \%$ of the $\mathrm{Cu}$ spiked into solution was removed within 20 minutes. Although the kinetics of adsorption vary between metals, so that these data cannot be directly applied to silver or lead, they do indicate that scavenging processes could certainly be responsible for removing significant dissolved phase metals from solution over the 20 minutes that each shear stress is maintained.

The suspended solids concentrations in the erosion chamber are far higher than would be expected to occur in Hingham Bay, thus it is not surprising that the scavenging time is so fast. In order to get a more realistic estimate of scavenging timescales, incubation experiments were performed on diluted chamber water. These results will be discussed in 
the Section 5.3.5.

\section{Colloidal Material}

To investigate the speciation of the metals released from the erosion chamber, in the June 2004 experiment the chamber water was also filtered with a $0.02 \mu \mathrm{m}$ filter in order to determine the colloidal and truly dissolved phases. Although this is still an operational definition, the additional filtration step does provide further information on the particle sizes with which the eroded materials are associated. Unfortunately the filter membrane had high iron blanks, so that the iron data all fall higher than any of the $0.45 \mu \mathrm{m}$ data and had to be discarded. For the other metals, the blank associated with filtering $10 \mathrm{mls}$ of seawater was applied to the measured concentrations and the corrections were all less than $10 \%$ of the total concentration. The data from the additional filtration step is shown on Figure 5-8. The fraction passing through a $0.45 \mu \mathrm{m}$ filter is referred to in the discussion below as 'dissolved', while species passing through the $0.02 \mu \mathrm{m}$ filter are considered 'truly dissolved', with colloidal species making up the difference between the two.

From Figure 5-8 the concentration of each metal passing through the $0.02 \mu \mathrm{m}$ filter is the same as that passing through the $0.45 \mu \mathrm{m}$ filter for lead, silver, copper and manganese until any concentration increase. This indicates that the metals in the Hingham Bay waters overlying the core are found in the dissolved phase rather than associated with colloidal material. This is expected for silver based on the findings of both Wen et al. [1997] and SanudoWilhelmy et al. [1996] who found that silver associated with the colloidal phase in Texas estuaries and San Francisco Bay respectively, decreased with increasing salinity. The distribution of copper reported among size fractions in coastal waters is variable. The colloidal fraction accounted for $50 \%$ of the dissolved copper concentration in San Diego Bay [Guo et al., 2000; Shafer et al., 2004]. In San Francisco Bay the colloidal fraction represented less than $10 \%$ of the total dissolved copper in marine waters [SanudoWilhelmy et al., 1996; Gee and Bruland, 2002]. These results suggest that even at similar salinities total dissolved copper in coastal, anthropogenically impacted waterbodies can exist in different phases. However data for Boston Harbor from nearby Quincy and Dorchester Bays also showed that the colloidal fraction was a negligible component of the total dissolved copper concentration [Kogut and Voelker, 2003].

The metals released immediately after the onset of erosion are in the dissolved phase. 
This is surprising considering the high concentrations of ideal colloid forming materials, organic carbon and metal oxide phases in the surfical sediments that are eroded. There is a distinct change in the nature of the eroded material at a shear stress of $0.22 \mathrm{Nm}^{-2}$, when there is a very strong release of iron, lead, copper and silver. Unlike at any other sampling point, there is a detectable colloidal fraction that is $27 \%, 51 \%$ and $77 \%$ of the dissolved silver, copper and lead respectively. By contrast all the manganese measured at this sampling point is in the dissolved phase. By the next shear stress increment $\left(0.3 \mathrm{Nm}^{-2}\right)$ the total dissolved fraction of silver, lead, copper and iron was significantly lower and equal to the dissolved fraction, indicating that all of the colloidal material detected earlier had been removed from solution.

Such a fast rate of colloid removal from solution was identified by Wen et al. [1997] who found that the transfer of trace metals between the colloidal phase and the particulate phase occurred with two distinct temporal rates, the first a fast rate operating over four hours and the second rate acting over 10 days. The removal of all colloidally associated metals between the shear stress steps of 0.22 and $0.26 \mathrm{~N}^{-2}$ is thus likely due to the aggregation of colloids released during resuspension.

Unlike silver, lead and copper, manganese does not show any association with the colloidal phase in the Hingham Bay June experiment at the $0.22 \mathrm{Nm}^{-2}$ point. Wen et al. [1997] found that the fraction of manganese in the colloid pool was an order of magnitude lower than for silver. They found that only after 9 days was significant manganese transferred to the particulate phase, likely due to the microbially mediated oxidation to $\mathrm{MnO}_{2}$. Manganese thus exists primarily in the dissolved phase in the absence of bacterial activity and was scavenged to only a limited extent even with the high suspended solids concentrations in the erosion chamber.

Why trace metals associated with colloids should only be identified at a single shear stress step is somewhat puzzling. However this observation is likely due to the experimental design, rather than being representative of the processes that occur during a sediment resuspension event in the environment. The concentrations of metals in the colloidal phase is determined by the competing processes of dissolved metal transfer to colloidal phases and the aggregation and removal of colloids to the particulate phase. The concentrations of particles in the overlying water of the erosion chamber, even with exchange of overlying water between shear stress steps, reached up to $4 \mathrm{~g} / \mathrm{kg}$, two to three orders of magnitude 
higher than is found in the waters of Boston Harbor and Massachusetts Bay (MWRA Weekly Monitoring Data). Due to the artificially high concentrations of particles the transfer of metals to the particulate phase will be the dominant process. Thus both the concentrations measured in the total dissolved phase and colloidal phase are lower bound estimates of the metal release to both of these phases. To try and get an improved estimate of the release to the total dissolved phase, the suspended solids concentration in the erosion chamber needs to be kept lower by continuous exchange of the overlying water. The problem with this is that it will lead to high levels of dilution, so that the total dissolved release may not be detected at all. An alternative approach may be to inject a tracer during the experiment, either a radiotracer [Wen et al., 1997] or low occurrence stable isotopes [Gee and Bruland, $2002]$, so that the extent of scavenging can also be quantified.

\subsubsection{Particle Release}

For each experiment, once the critical shear stress for erosion was exceeded, increasing the shear stress further led to increasing amounts of trace metals associated with the particulate phase being eroded into the water column of the erosion chamber. The total amount of trace metal at each stress level is shown in Figure 5-12. Iron, manganese, lead, silver and copper all show a smoothly increasing flux as the shear stress increases beyond the threshold of exosion for both Hingham Bay experiments. The total amount of metal eroded in October 2003 is consistently higher than in June, with the October fluxes ranging from an average of 1.3 times higher for silver and 2.1 times higher for $\mathrm{Mn}$ and $\mathrm{Pb}$. Lead and barium do not show the same monotonic increase, but rather at the final shear stress of $0.3 \mathrm{Nm}^{-2}$ there is actually a decrease in the total amount of lead and barium in suspension. The Massachusetts Bay data also show a smooth increase in solid phase metals after the erosion threshold has been exceeded and the total amounts of each metal eroded becomes constant at higher shear stresses.

Although the total amount of solid phase metal resuspended generally increased with increasing shear stress, the concentration of metals in the eroded particles (in $\mu$ mol(metal)/g(eroded sediment)) changed with each shear stress step. This change is shown in Figure 5-13. There is considerable variation in the solid phase concentration over the range of shear stresses for each metal, indicating that as erosion progresses sediment particles with different levels of metal enrichment are eroded. 

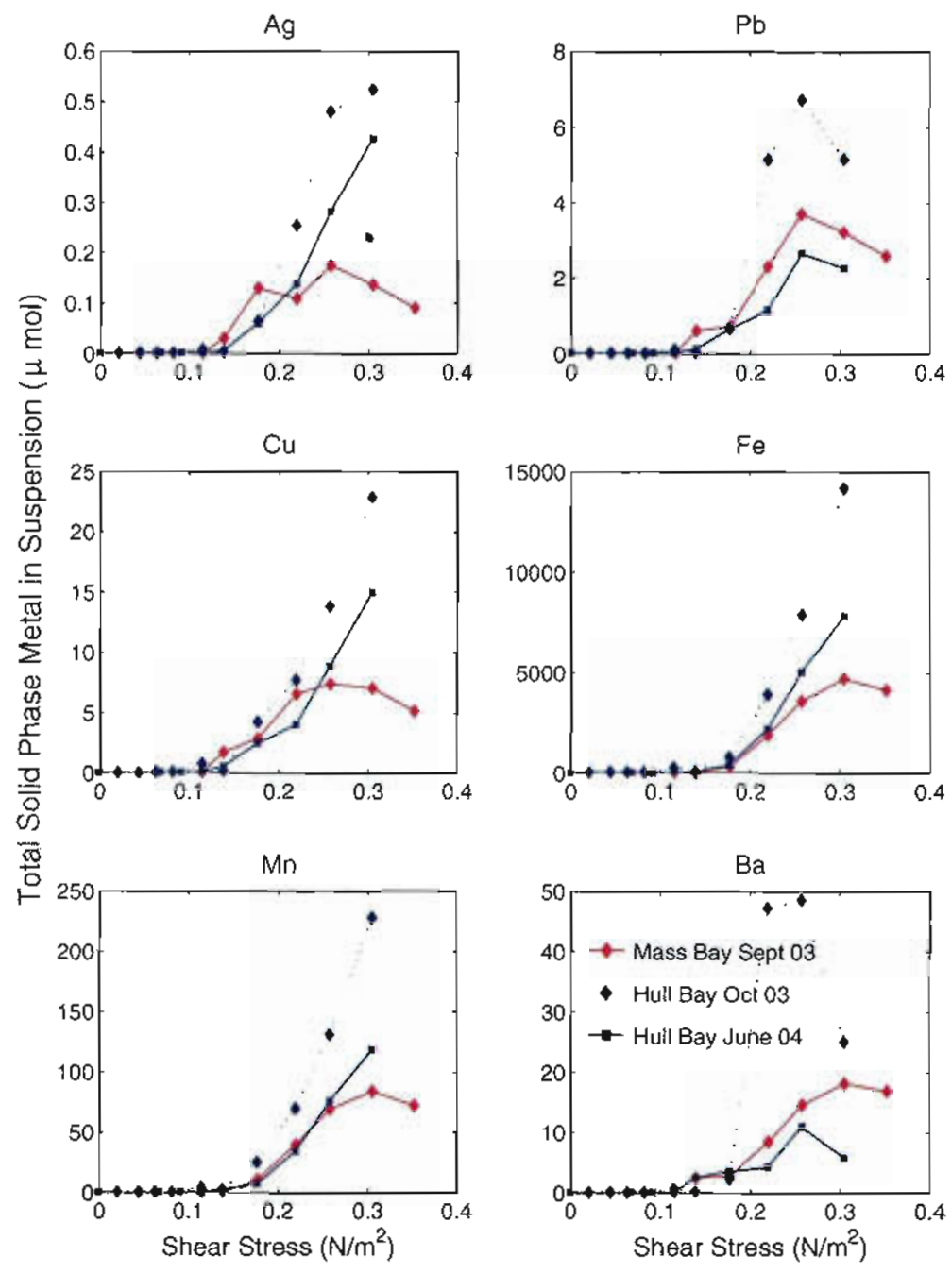

Figure 5-12: The total amount of particulate trace metals resuspended in the erosion chamber for each experiment. 
For copper and lead the highest concentration particles are eroded at or just beyond the critical erosion threshold. Beyond $0.2 \mathrm{Nm}^{-2}$ the concentrations fall with continuing erosion. The only exception to this is the lead behavior in October 2003, where the peak in concentration occurs at $0.21 \mathrm{Nm}^{-2}$, several shear stress steps beyond the erosion threshold. The silver in Massachusetts Bay shows a similar response, with the highest concentrations eroded just after the erosion threshold. Similarly in Hingham Bay the silver concentrations peak close to the erosion threshold, but the concentrations in the particulate phase remain constant with continued erosion.

The concentration variation of iron is different, with the concentration rising gradually after the erosion threshold and then reaching a steady, maximum level beyond $0.26 \mathrm{Nm}^{-2}$ for both Hingham Bay experiments and falling for the Massachusetts Bay experiment. The manganese data shows quite different responses in each experiment. The Hingham Bay 2003 data show a gradual rise in Mn concentration while the Massachusetts Bay data and the 2004 Hingham Bay data show a sharp peak in Mn concentration at the erosion threshold. In both Hingham Bay experiments the Mn concentration reaches a steady level by 0.26 $\mathrm{Nm}^{-2}$, while in Massachusetts Bay the Mn concentration falls at higher shear stresses. The barium behavior is also variable between experiments, with the highest concentration material resuspended at the erosion threshold in Massachusetts Bay and Hingham Bay in June, but not in October.

Both Hingham Bay experiments show some release of iron and manganese prior to the erosion threshold, likely associated with the particles that were visually evident in the water column before the onset of bulk erosion. There are peaks in silver, lead and copper that coincide with these pre-threshold iron and manganese peaks, suggesting that the trace metals are associated with the readily erodible iron and manganese phases.

The change in suspended metal concentration measured as a function of shear stress in the erosion chamber can be converted into a concentration variation with erosion depth, assuming the sediments erode in a uniform manner across the entire sediment surface. Distinct variations in concentrations thus occur as progressively more sediment is eroded. As the particles are all derived from the upper layers of the sediment, it is expected that the concentrations measured in the erosion chamber must bear some relationship to the metal concentration measured in the sediment. The measured concentration of metals in the sediments was obtained from high resolution sectioning of the sediment. Even with the 


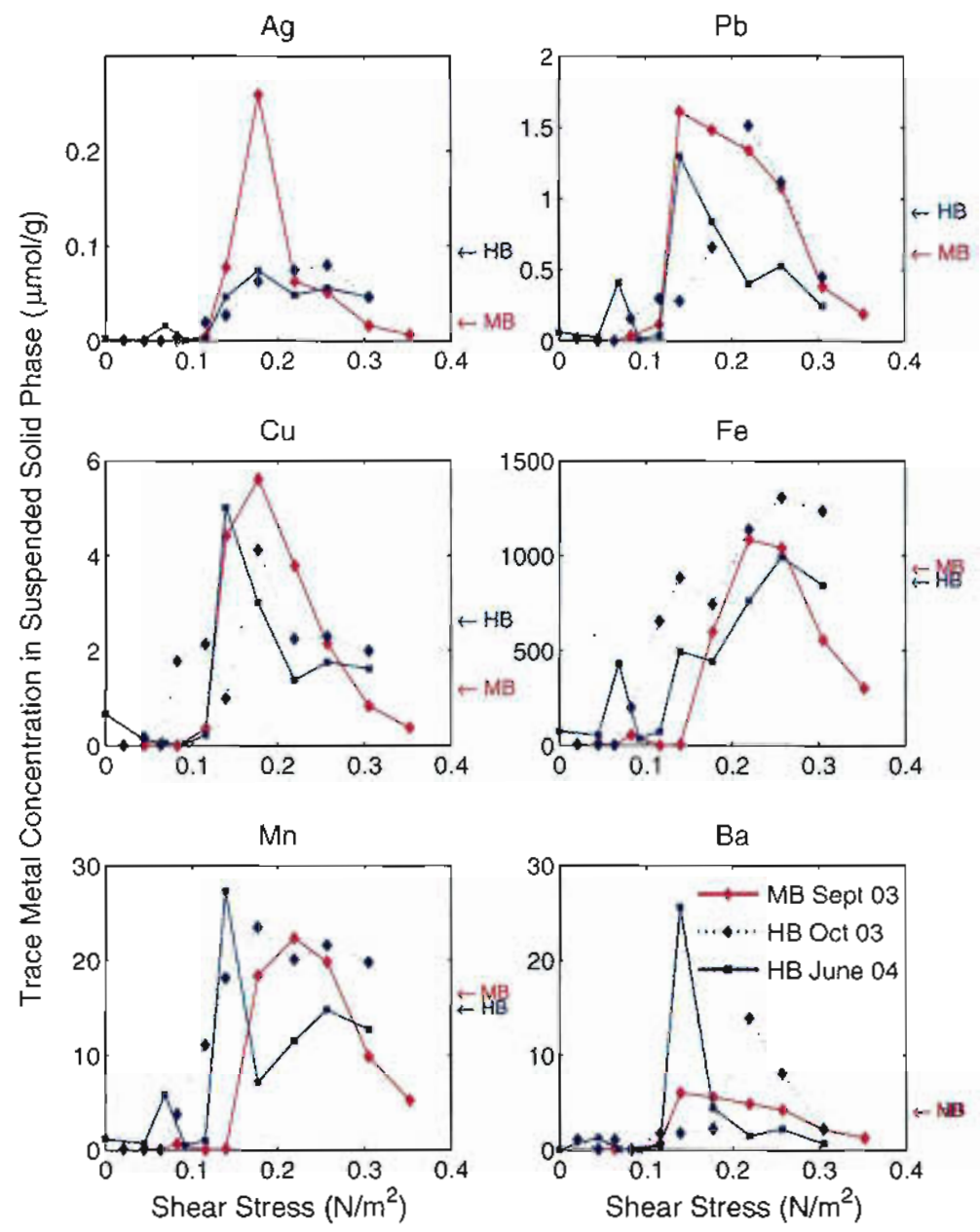

Figure 5-13: Concentration of trace metals in the eroded solid phase as a function of shear stress. The arrows on the right hand side of each plot are the solid phase metal concentration measured in the chamber 8 hours after completion of the experiment. The arrows thus represent the concentration of material that takes over 8 hours to settle. The arrow labeled 'MB' refers to the Massachusetts Bay September 2003 experiment and the arrow labeled 'HB' is for Hingham Bay June 2004. 
Table 5.6: Surface Solid Phase Concentrations for Both Sites

\begin{tabular}{|c|c|c|c|c|c|c|c|c|}
\hline \multicolumn{2}{|c|}{ Section Depth } & \multicolumn{7}{|c|}{ Solid Phase Concentration } \\
\hline $\begin{array}{l}\text { Start } \\
(\mathrm{mm})\end{array}$ & End & $\begin{array}{c}\mathrm{Fe} \\
(\mathrm{mm}\end{array}$ & $\begin{array}{r}\mathrm{Mn} \\
\mathrm{l} / \mathrm{kg})\end{array}$ & $\mathrm{Ag}$ & $\begin{array}{l}\mathrm{Pb} \\
(\mathrm{mr}\end{array}$ & $\begin{array}{c}\mathrm{Cu} \\
\mathrm{ol} / \mathrm{kg})\end{array}$ & $\mathrm{Ba}$ & $\% \mathrm{OC}$ \\
\hline \multicolumn{9}{|c|}{ Hingham Bay Jan 2002} \\
\hline 0 & 3 & 752 & 12.5 & 20 & 381 & 1076 & 3307 & 3.29 \\
\hline 3 & 6 & 775 & 12.6 & 21 & 387 & 1094 & 3343 & 3.21 \\
\hline 6 & 12 & 795 & 12.0 & 22 & 389 & 1158 & 3379 & 2.86 \\
\hline \multicolumn{9}{|c|}{ Hingham Bay July 2002} \\
\hline 0 & 4 & 743 & 11.7 & 25 & 372 & 1103 & 3270 & 2.98 \\
\hline 4 & 8 & 747 & 11.4 & 25 & 374 & 1108 & 3321 & 2.9 \\
\hline 8 & 12 & 722 & 11.0 & 21 & 372 & 1116 & 3307 & 2.89 \\
\hline \multicolumn{9}{|c|}{ Hingham Bay September 2002} \\
\hline 0 & 4 & 736 & 12.0 & 25 & 373 & 1116 & 3183 & 3.14 \\
\hline 4 & 7 & 718 & 11.1 & 26 & 377 & 1081 & 3197 & 2.88 \\
\hline 7 & 10 & 723 & 11.1 & 26 & 384 & 1138 & 3234 & 2.77 \\
\hline \multicolumn{9}{|c|}{ Massachusetts Bay October 2001} \\
\hline 0 & 3 & 654 & 10.2 & 5.5 & 339 & 681 & 3270 & 2.27 \\
\hline 3 & 6 & 691 & 10.3 & 5.5 & 341 & 721 & 3409 & 2.37 \\
\hline 6 & 9 & 685 & 10.2 & 5.7 & 353 & 713 & 3377 & 2.33 \\
\hline 9 & 10 & 691 & 9.9 & 6.4 & 361 & 727 & 3430 & 2.41 \\
\hline \multicolumn{9}{|c|}{ Massachusetts Bay February 2002} \\
\hline 0 & 3 & 662 & 11.3 & 5.7 & 344 & 674 & 3292 & 2.27 \\
\hline 3 & 6 & 675 & 10.5 & 6.1 & 353 & 740 & 3314 & 2.37 \\
\hline 6 & 9 & 650 & 9.8 & 6.1 & 351 & 749 & 3336 & 2.33 \\
\hline \multicolumn{9}{|c|}{ Total Variation (\%CV) } \\
\hline Hingham Bay & & 3 & 5 & 9 & 2 & 2 & 2 & 6 \\
\hline Massachusetts & Bay & 3 & 5 & 6 & 2 & 4 & 2 & 2 \\
\hline
\end{tabular}

best fine scale sectioning, a resolution in the surface sediments of only $3 \mathrm{~mm}$ was possible. Shear stresses up to $0.25 \mathrm{Nm}^{-2}$ are thus only eroding sediments from within the very first sediment section. To compare the concentrations measured in the suspended particles with those expected based on the metal concentrations in the sediments, the suspended solids concentration data is normalized by concentration data from sectioned sediment cores. Unfortunately solid phase concentrations on sediment cores collected at the same time as the erosion cores are not currently available. Table 5.6 contains a compilation of the surface solid phase data that is available for the two sites. It is evident that the variation in the top $1 \mathrm{~cm}$ for a year of data for Hingham Bay and 3 months for Massachusetts Bay is less than $10 \%$ for each element of interest. Due to this minimal variation it is appropriate to normalize the data by sediment collected at a different time in order to compare the concentrations measured in the erosion chamber particles with the bulk sediment concentrations. The normalized data is shown in Figure 5-14. 

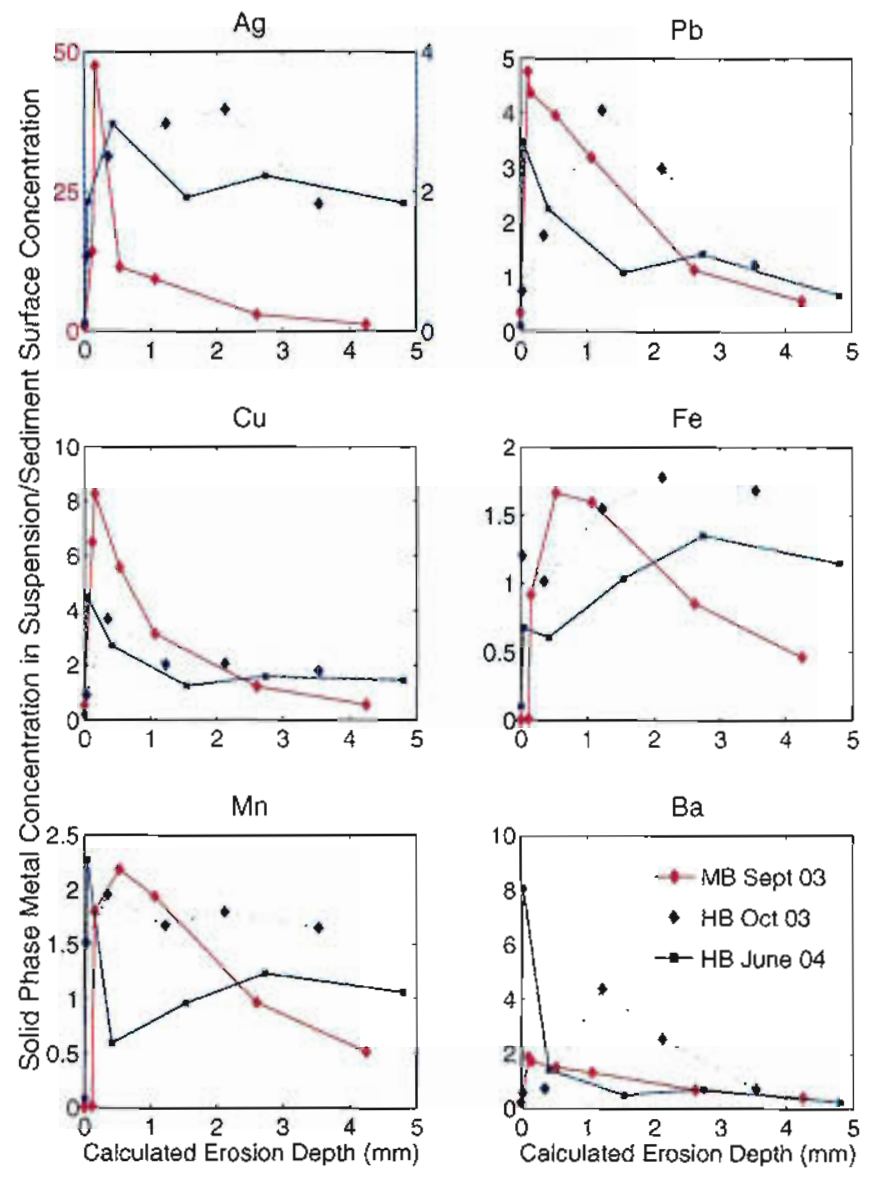

Figure 5-14: Metal concentrations of suspended solids vs calculated erosion depth. The concentrations are normalized by the concentration measured in the $0-3 \mathrm{~mm}$ section of sediments sampled from the same site. The Hingham Bay data is normalized by the concentration of September 2002 Core 2 data and the Massachusetts Bay data by Core 3 October 2001. Note that for the silver plot the data for Massachusetts Bay is plotted against the left hand axis, while the Hingham Bay data is plotted against the right hand axis. 
From Figure 5-14 it is evident that for copper as well as lead and silver in all but the Hingham Bay 2003 experiment, that particles enriched over the bulk phase erode first, and that as erosion progresses the level of enrichment decreases. Cantwell et al. [2002] and Calvo et al. [1991]also reported enrichments above the bulk concentration in resuspended sediments. The level of enrichment for the Massachusetts Bay samples is greater than for Hingharn Bay. Iron and manganese do not show such distinct enrichment as the trace metals. As erosion progresses and the depth of erosion reaches the depth at which the sectioned sediments were sampled, it is expected that the normalized concentrations shown in Figure 5-14 would equal one. Because the sediment concentrations were not determined on the sediments that were used in the erosion experiment, it cannot be expected that the normalized concentration would be exactly one. However, considering the possible temporal and spatial variability, the concentrations of most metals tend to the bulk concentration. The exceptions are silver and copper, which in both Hingham Bay experiments stabilize at enrichment factors of around 2. That most metals tend towards a value of one suggests that the erosion chamber is resuspending bulk sediments at higher shear stresses. However the silver and copper data may indicate that even at higher shear stresses the erosion is not uniform across the sediment surface or that there is some preferential erosion of particles enriched in silver and copper.

Potential processes that could be responsible for the enrichment observed in the most readily erodible sediments at both sites include both physical and chemical mechanisms. The physical mechanisms could be due to the preferential association of metals with the finer, more readily erodible particles within the sediments. Due to different site locations there may be a difference in the trace metal concentration being delivered to each site. The chemical mechanism that could be responsible may be a diagenetic mechanism that focuses metals right at the sediment-water interface.

Finer grained material are generally more enriched in metals [Bothner et al., 1998]. As particle size decreases there is an increase in the surface area to volume ratio, so that surface processes such as adsorption and surface complexation are enhanced. If fine grained particles are preferentially suspended, a winnowing effect would account for the enrichment in the most readily eroded particles. As the shear stress increases there is less preferential erosion and bulk sediments are resuspended and the enrichment factor decreases as the more concentrated particles are diluted by bulk material. The preferential erosion of fine grained 
particles was used by Cantwell et al. [2002] to explain the differences in suspended particle enrichment observed between two sites in Narragansett Bay. The same argument can be used to explain the differences in enrichment between Hingham Bay and Massachusetts Bay; the Massachusetts Bay site has a higher proportion of sand, so that during erosion a disproportionate amount of fine material was eroded and was thus more highly enriched. Hingham Bay, with a more uniform grain size did not have as much preferential erosion, so does not report such a strong enrichment.

Alternatively the differences in the most readily erodible material may simply reflect newly arrived matter that has reached the sediment-water interface but not been incorporated into the sedimentary matrix. The different sedimentary environments and locations relative to potential sources will certainly lead to variations in the trace metal concentrations reaching each site. However a comparison of the eroded material composition with that of the USGS sediment trap data from Feb. 13 - May 24, 2001 (courtesy M.Bothner, USGS) moored $2.4 \mathrm{~km}$ south-east of the MB sediment sampling site indicate that the two materials are quite different. The peak concentrations of $\mathrm{Ag}, \mathrm{Pb}, \mathrm{Cu}$ and $\mathrm{Fe}$ eroded from the Massachusetts Bay site are $37 \pm 24,4.7 \pm 1,9.5 \pm 1$ and $0.9 \pm 0.2$ times higher than the concentrations measured in the sediment traps. The uncertainty given in these figures is due to the variability in concentrations measured between cylindrical and funnel traps. The material collected in the sediment traps is not a perfect indication of the material reaching the seafloor at the MB site as the traps are located $4 \mathrm{~m}$ above the seafloor and continuing remineralization as well as hydrodynamic sorting of particles can generate a fractionation between the material in the sediment traps and the material that is deposited. The trap data is also from a limited time period and variability in outfall discharge [Ravizza and Bothner, 1996] may also lead to differences. However the trap data does at least give some indication of the nature of the material reaching the $\mathrm{MB}$ site, and a comparison of the concentrations suggests that silver, copper and lead are enriched in the most readily erodible sediments relative to settling material, while $\mathrm{Fe}$ is not. It thus seems unlikely that the uppermost eroded layers represents unprocessed settling material as trace metal concentrations in the upper layers are considerably higher than was reported in the sediment traps.

A diagenetic process transferring metals from the porewaters to the solid phase at the sediment-water interface is another possible mechanism leading to the formation of the enriched layer. Porewater profiles of iron and manganese measured in Hingham Bay in 
June 2004 indicate that there should be a flux of these metals to the overlying water (see Chapter 3). However benthic chambers deployed at the same time only record a flux of manganese and not iron. This suggests that there is also a finer scale structure in porewater concentrations than could be detected over the 0-3 mm sediment sectioning range. Rapid, sub-mm scale changes in the porewater concentrations of metals close to the sedimentwater interface have also been identified by others [Brendel and Luther, 1995; Davison et al., 1997]. For there to be no iron flux measured, the Fe concentration at the interface must be essentially zero. The Fe in the porewaters must be trapped at the sediment-water interface, thus preventing the flux of iron to the overlying water. There is a manganese flux measured in the chamber as the oxidation of manganese is slower than that of iron [Landing and Bruland, 1987], so that manganese is less effectively internally recycled. The fluxes expected from the Mn porewater profiles, however, are greater than measured in the chambers, indicating that there is also some trapping of Mn. Similar discrepancies between calculated $\mathrm{Fe}$ fluxes expected from porewater profiles and benthic chamber data have been measured at the Massachusetts Bay site (J. Morford, pers comm.), suggesting that an iron oxide layer also exists at the sediment-water interface at the MB site. Despite the formation of an oxide layer at the interface, the iron and manganese in the eroded sedirnents do not show as strong an enrichment in the suspended particles as the trace metals do. This is because the considerably higher background concentrations of these metals obscure the diagenetic signals.

The trapped iron oxides at the sediment-water interface will scavenge trace metals diffusing towards the sediment-water interface, thus enriching the metals at the interface [Sundby et al., 1986; Westerlund et al., 1986]. Analysis of the benthic chamber deployed in Hingham Bay in January 2002 detected no evidence of a flux of silver or copper, despite the porewater profile indicating that there should be. There thus seems to be evidence from a combinar tion of porewater sampling and benthic chamber measurements that there is a very fine scale removal of metals from porewaters at the surface of the sediments. This mechanism may be forming the layer of enrichment that has been detected in the uppermost erodible sediments.

The particles initially eroded from the Massachusetts Bay site were more enriched than those from Hingham Bay. A diagenetic mechanism may also account for this observation. The key difference between the sites is that the Hingham Bay site is more reducing. Sulfide 
was never detected at the Massachusetts Bay site but is detected by $7 \mathrm{~cm}$ at Hingham Bay. It is likely, based on observations at other coastal sites [Canfield, 1989], that sulfate reduction is active throughout most of the Hingham Bay sediment column even though no sulfide is detected in the surface porewaters. At both sites, as the surface-enriched layer is moved downwards by continued sedimentation or mixing, the particles encounter a sub-oxic environment. Reductive dissolution of the iron or manganese oxides that the trace metals are associated with will lead to a release of the metals to the porewaters. At the Hingham Bay site due to the presence of trace levels of sulfide, the metals will be precipitated as insoluble sulfide phases. At the $\mathrm{MB}$ site there is no sulfide so the sink removing metals from the porewaters into a reduced phase does not exist. The solid phase profiles from each site are shown in Figure 5-15. In Massachusetts Bay there is an obvious surface layer, about $2 \mathrm{~cm}$ deep in which all of the metals shown are enriched. This is because a significant proportion of the metals released to the porewaters must re-adsorb onto the metal oxide layer that persists at the surface. The surface oxic layer in MB thus becomes continually concentrated with metals by a reduction/oxidation cycle. In Hingham Bay the enriched layer is not as distinctive in the solid phase profiles indicating this concentrating mechanism does not happen to the same extent. This is because a smaller fraction of the released metals re-adsorb to the oxides as they precipitate as sulfide instead. Due to this reduced concentrating mechanism the degree of enrichment observed in Hingham Bay is less than that in Massachusetts Bay.

A combination of winnowing of the fine grained particles as well as erosion of the diagenetically formed surface enriched layer in the sediments is likely to be responsible for the high concentrations of trace metals that are initially eroded at both sites. Whatever the mechanism, the erosion of enriched particles at low shear stresses increases the impact of sediment resuspension on remobilizing metals from coastal sediments. Low shear stresses will occur more frequently and so regular erosion of concentrated particles may be an important mechanism of remobilization. This is investigated in more detail in Section 5.3.7.

\section{Metal Concentration in Slowly Settling Material}

The metal concentration of material that remained in suspension 8 hours after the erosion experiments had ended is shown in Figure 5-13. After 8 hours, $9 \%$ and $2 \%$ for Hingham Bay and Massachusetts Bay respectively of material eroded at a shear stress of $0.3 \mathrm{Nm}^{-2}$ 


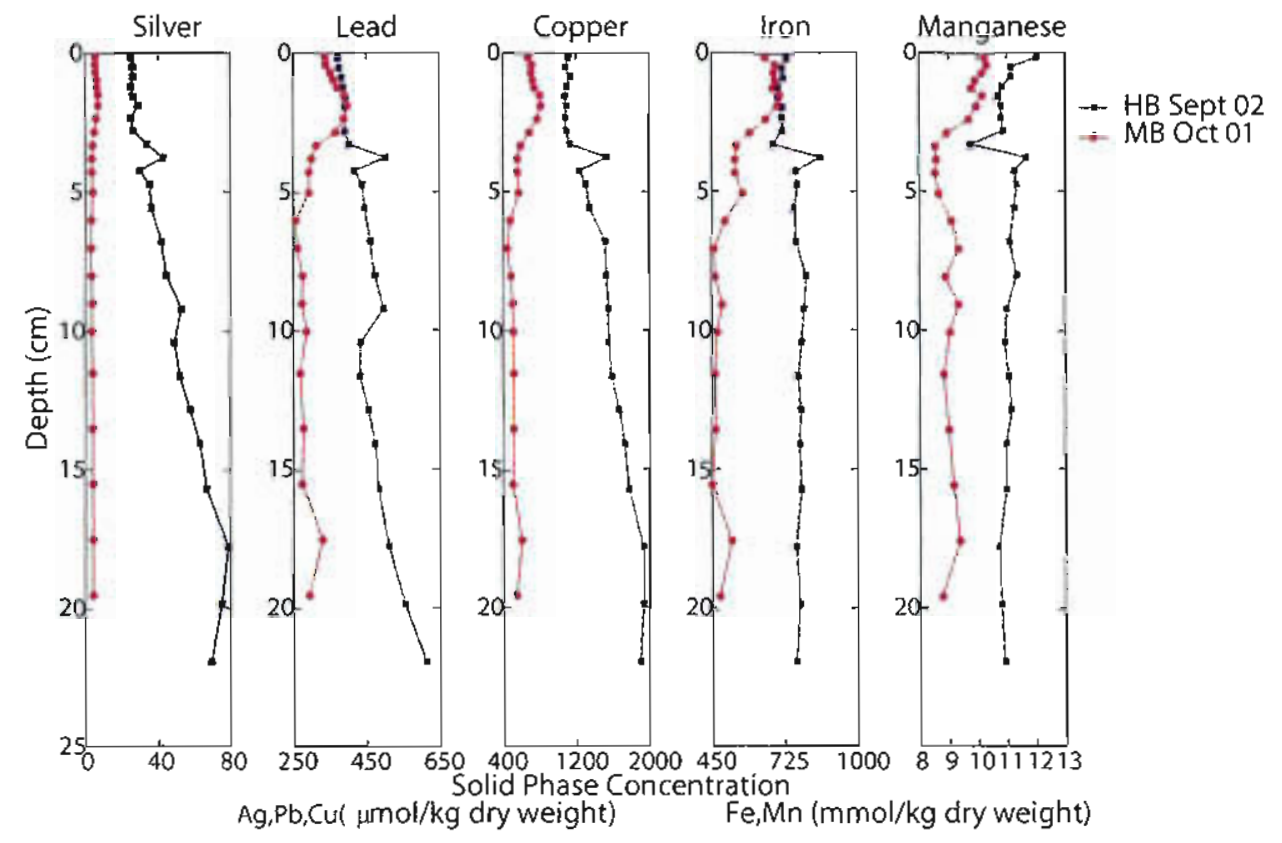

Figure 5-15: Solid phase profiles of for silver, lead, copper, iron and manganese for Massachusetts Bay October 2001 and Hingham Bay September 2002).

remained in solution. The Hingham Bay site thus contains a higher proportion of fine, slowly settling material than the MB site, consistent with the fact that The Hingharn Bay site has a higher proportion of silt, clay and organic carbon (Table 5.1). However direct comparison of these percentage values is not an appropriate way of comparing the sediment types at each site. The erosion chamber for the Massachusetts Bay experiment was taken to a final shear stress of $0.4 \mathrm{Nm}^{-2}$ and the final suspended solids concentrations were twice as high as those in Hingham Bay. Due to the higher particulate concentration, greater coagulation of the fine particles may have occurred so stripping the water of finer particles.

For the Hingham Bay samples the metal concentrations of the fine material are higher than the bulk surface sediment and in the case of silver and lead the concentrations remaining in solution are comparable to the peak concentrations measured during the erosion experiment. The Massachusetts Bay site samples are all above the bulk surface sediments concentrations but are not at levels as high as the peak concentrations observed during the erosion experiment.

That the particles that remain in suspension over timescales of hours are enriched in metals relative to the bulk sediments from which they were derived is an important result, 
as it is these particles that have the longest residence time in the water column. Sediment resuspension events can thus result in a dispersal of the fine particles which are enriched in trace metals. For the Hingham Bay site the fractions of trace metal in suspension after 8 hours compared to the total quantity of particulate metal resuspended at a shear stress

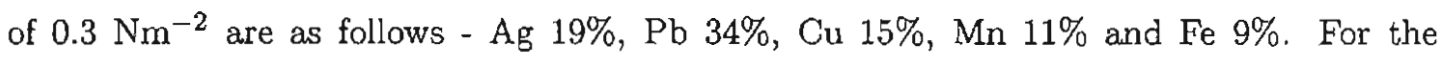
Massachusetts Bay site the fraction is between 2 and $4 \%$ for these same metals. The large proportion of metals still in suspension from the Hingham Bay site is due to the high proportion of fine material still in suspension and the enriched metals concentrations in the particles.

Additional experiments to confirm if this high proportion is a reproducible feature and to assess if there are variations in the ratio in different seasons are required. The proportion of metals retained in suspension in late fall to early spring is especially important, as this is when most storms and thus resuspension events occur. Settling experiments on the Massachusetts Bay site sediments should also be done starting at a more realistic suspended solids concentration to assess if the difference between sites is due to the experimental details or is an actual feature. These preliminary results however suggest that resuspension events are very important at the Hingham Bay site, as a significant proportion of the total flux given stays in suspension on timescales important for transport and chemical reactions.

\subsubsection{Incubations}

In order to assess the longer term behavior of eroded material under more realistic suspended particle concentrations than those generated in the erosion chamber, incubation experiments were undertaken on the water samples from the erosion chamber. For each incubation with eroded particles, a control experiment was also done. The control was used to assess the role of particles in releasing material, and so contained particle-free water, generated by $0.45 \mu \mathrm{m}$ filtration of water from the erosion chamber. The control was stirred and sampled in the same manner as the incubations containing particles. The results for the Hingham Bay October 2003 experiment are shown in Figure 5-16: The October incubations were collected after the $0.3 \mathrm{Nm}^{-2}$ shear stress step. Unfortunately the water samples collected for metal analysis were contaminated and so only the nutrient data is shown in Figure 516. Only the first four samples collected for ammonium and phosphate were analyzed due to a miscommunication. The results for the June 2004 erosion experiment are shown in 

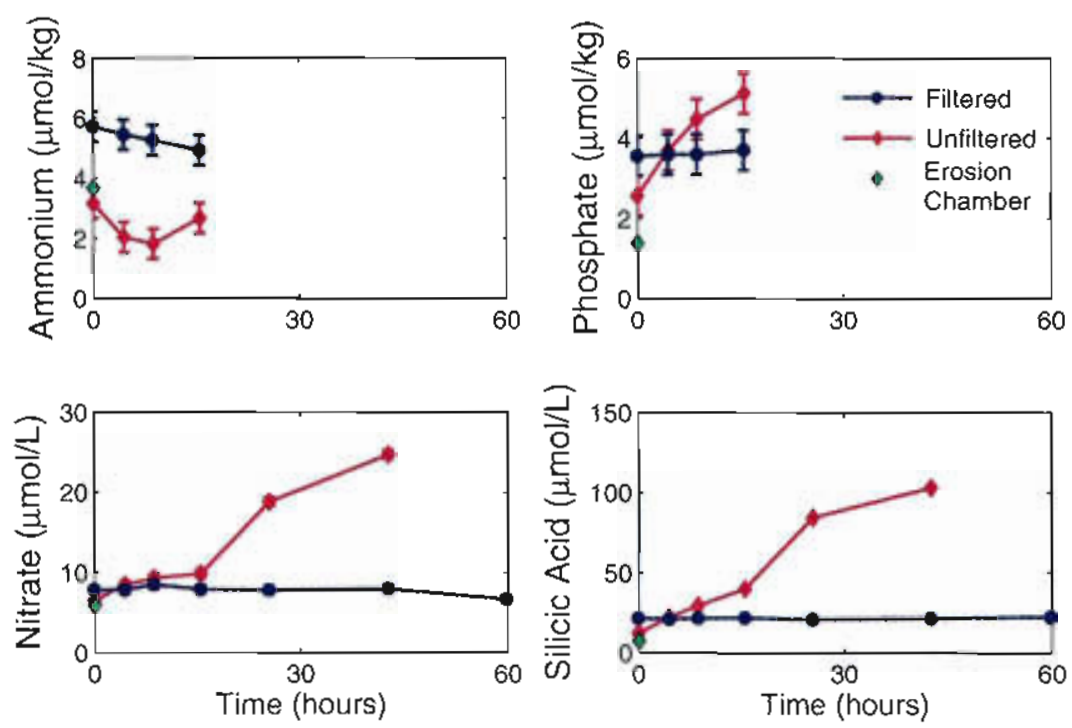

Figure 5-16: Incubation results for October 2003 particles resuspended at a shear stress of $0.3 \mathrm{Nm}^{-2}$. Error bars are the standard deviation of the analysis.

Figures 5-17 and 5-18. The particles incubated were collected at two shear stresses, 0.18 $\mathrm{Nm}^{-2}$ and $0.3 \mathrm{Nm}^{-2}$. The particles collected were diluted to the total suspended solids concentrations of 167 and $138 \mathrm{mg} \mathrm{kg}{ }^{-1}$. These TSS concentrations are still higher than the TSS values in the Harbor, which generally fall below $20 \mathrm{mg} \mathrm{kg}^{-1}$. A greater dilution was not possible as any release from the particles would not have been detected. Nitrate and silicic acid samples collected after 30 hours were unfortunately not analyzed.

The nutrient incubation results show a distinct difference between the unfiltered and 0.45 $\mu \mathrm{m}$ filtered treatments for both $0.3 \mathrm{Nm}^{-2}$ incubations. The unfiltered treatments release both phosphate and silicic acid to solution, while the filtered treatments show essentially no change. The ammonium values for both treatments decline to zero in June 2004, and the limited data available for October 2003, suggest that the ammonium data was also declining. There is a strong, delayed release of nitrate in the unfiltered October 2003 incubation. Unfortunately there is insufficient data in June to be able to identify a similar delayed release of nitrate. In the low shear stress incubation in June 2004 the concentrations of phosphate, nitrate and silicic acid in both treatments remained constant over the time data was available, while ammonium dropped. For both ammonium and phosphate the concentrations of the first sampling point for the two treatrnents is different, with the 

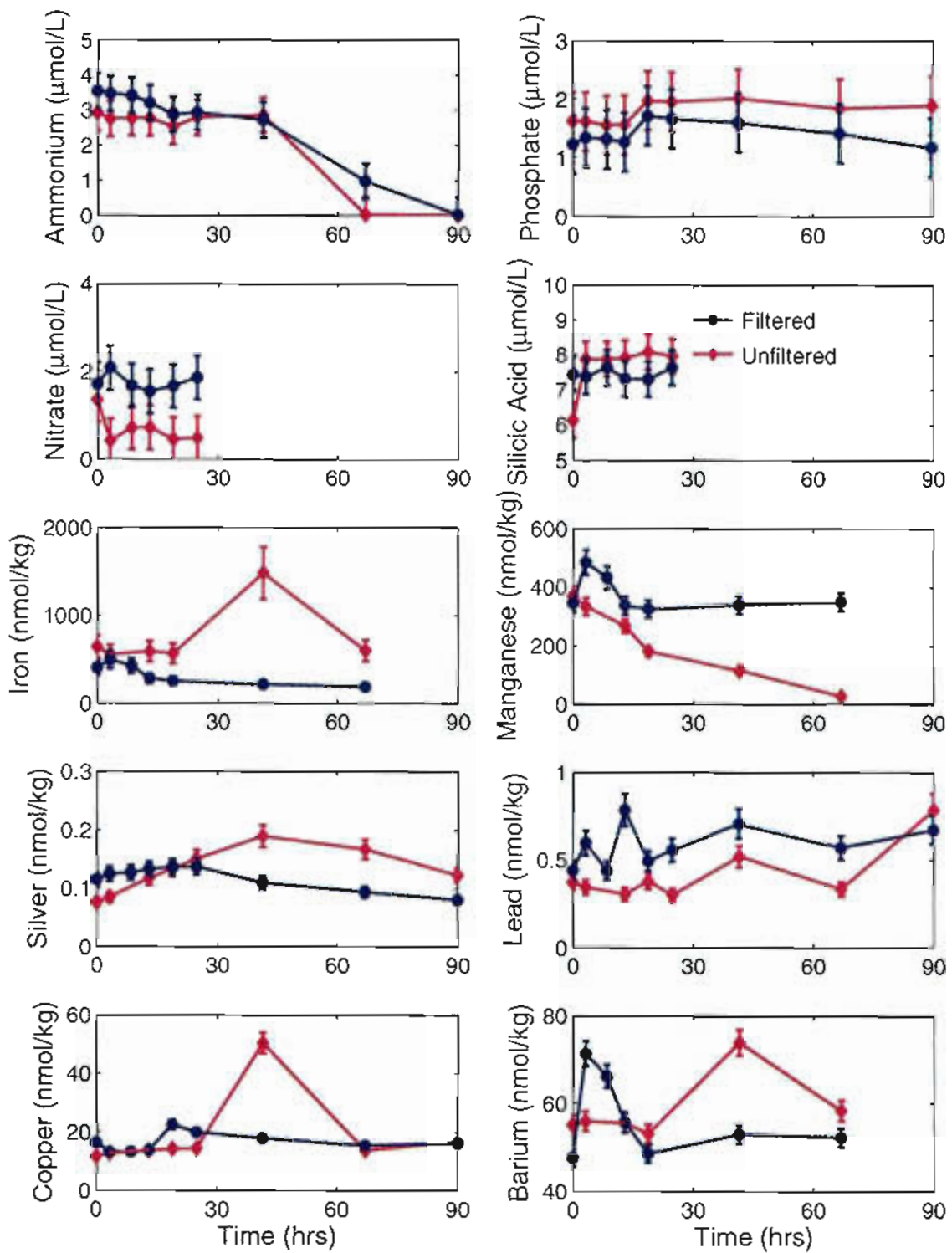

Figure 5-17: Incubation results for June 2004 particles resuspended at a shear stress of 0.18 $\mathrm{Nm}^{-2}$ with an incubating suspended solids concentration of $167 \mathrm{mg} \mathrm{L}^{-1}$. Error bars are the standard deviation of the analysis. 

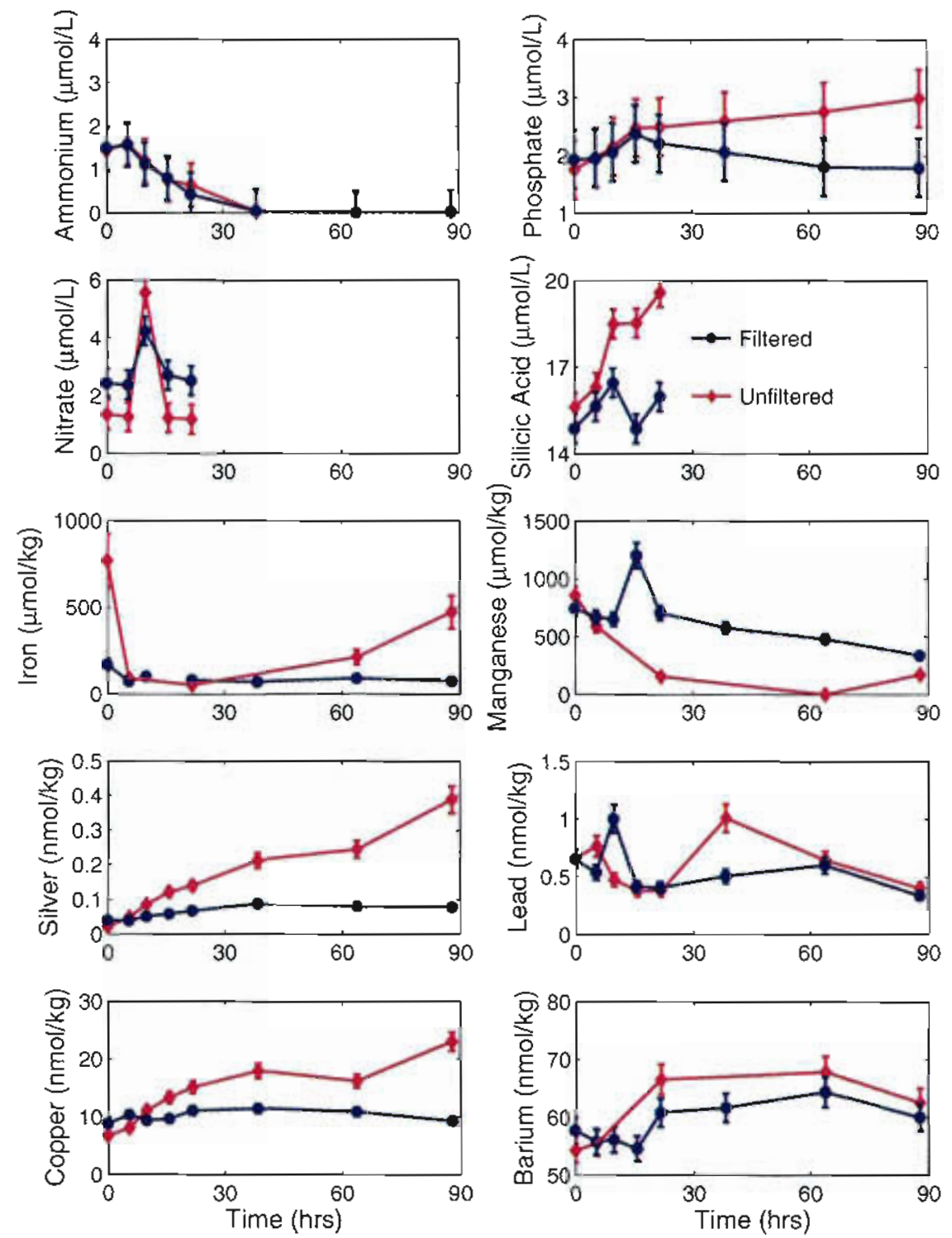

Figure 5-18: Incubation results for June 2004 particles resuspended at a shear stress of 0.3 $\mathrm{Nm}^{-2}$ with an incubating suspended solids concentration of $100 \mathrm{mg} \mathrm{L}^{-1}$. Error bars are the standard deviation of the analysis. 
filtered concentration being higher. Due to the high particulate load the filtration processes took half an hour. During this time the solids accumulating on the filter paper may have released additional nutrients, so that the filtered samples started with higher concentrations. To avoid this in the June 2004 incubation experiments, several filtering rigs were used simultaneously, so that filtration was much faster.

The marked differences between treatments indicate that suspended solids are releasing additional nutrients to solution. This is certainly the case for silicic acid and phosphate. The release of phosphate may be due to continuing ion-exchange reactions. The increase in silicic acid may be due to enhanced dissolution of opal due to more dynamic flow conditions [Koschinsky et al., 2001]. Ion-exchange is an unlikely mechanism for the release of Si as the $\mathrm{pK}_{a}$ of $\mathrm{H}_{2} \mathrm{SiO}_{4}$ is 9.5 in seawater, so almost $100 \%$ of the $\mathrm{H}_{2} \mathrm{SiO}_{4}$ is not ionized and so will not participate in ion-exchange reactions. The loss of ammonium may be due to either volatilization (due to stirring), oxidation to nitrate or re-adsorption to solids. At the $\mathrm{pH}$ of seawater, as the solution was stirred rather than bubbled with a purging gas, volatilization is unlikely to be the dominant removal mechanism. Adsorption to solids cannot be the removal mechanism as the loss of ammonium occurred in both filtered and filtered treatments. Oxidation to nitrate seems the most likely, especially considering the large increase in nitrate after 20 hours observed in the October incubation. Unfortunately the inorganic nitrogen data set is incomplete, so a mass balance calculation is not possible and so the processes operating are difficult to determine.

The total releases of nutrients to the incubations for the June experiments are shown in Table 5.7. The incubation of the particles from the higher shear stress level released considerably more phosphate and silicic acid to solution than the low shear stress incubation. The particles eroded from a few mm deeper within the sediment column are exposed to higher concentrations of porewater and also have had a longer residence time in the sediment. The porewater concentrations of phosphate and silicic acid are 4 and 1.7 times higher in the $2.5-5 \mathrm{~mm}$ depth range compared to the $0-2.5 \mathrm{~mm}$ range. For phosphate a higher concentration of ions will be adsorbed to the deeper particles, so there will be a greater release to the dissolved phase. The increase in porewater concentration, however, does not account for all of the differences in release between the two incubations, and ion-exchange is unlikely to be important for silicic acid. The reason for the large difference in release is unknown but must be due to each incubation having particles with different reactivities. 
Table 5.7: Dissolved Phase Release from Incubated Particles after 90 Hours

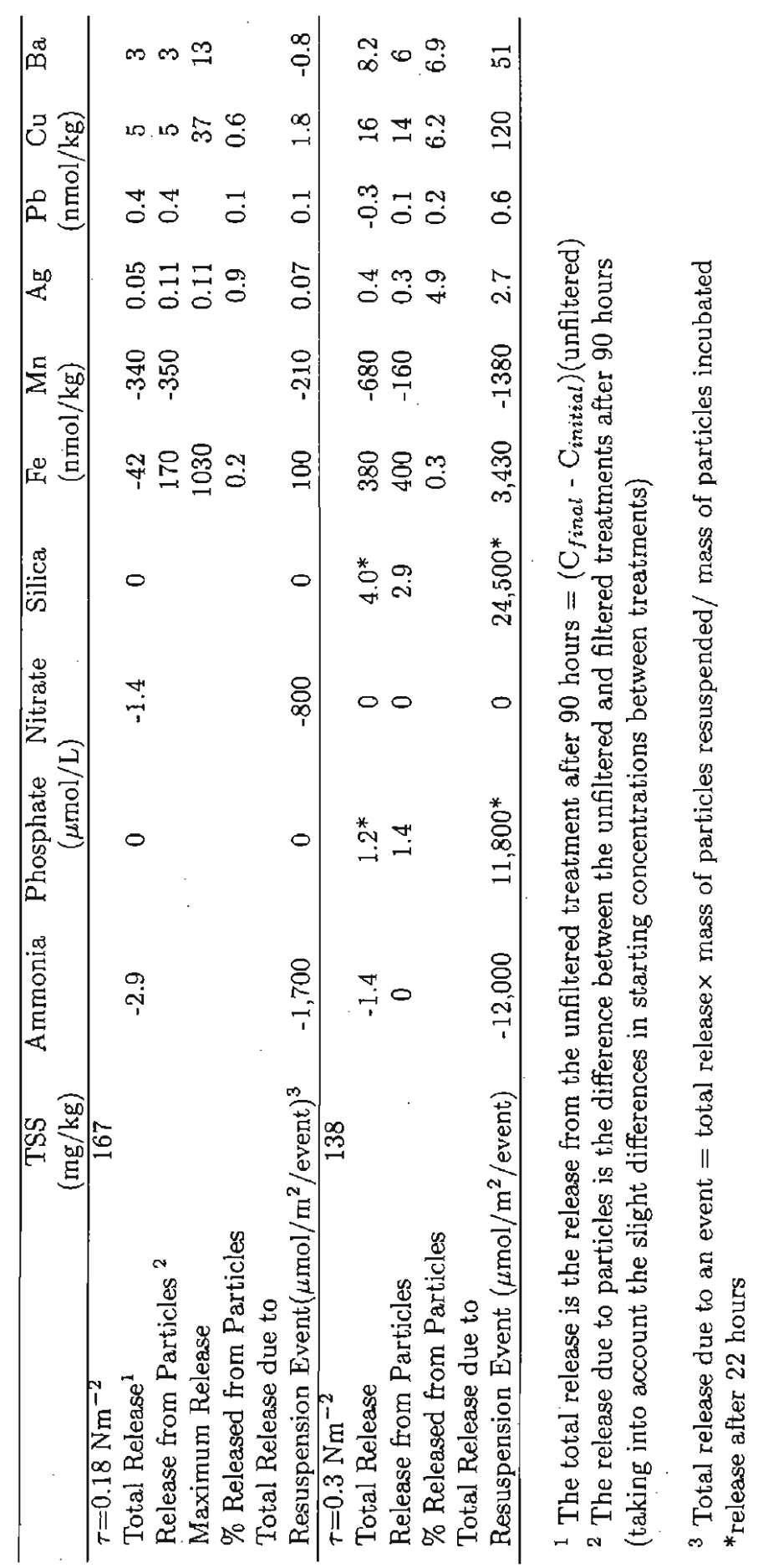


The total release of metals measured in each incubation experiment are also given in Table 5.7. In both June incubations, the manganese concentration in the unfiltered treatments declined to a level close to zero, while the filtered samples remain at constant or only slightly declining concentrations over the 90 hour experiment. This is consistent with the oxidation of manganese being slow but enhanced by heterogeneous catalysis on mineral surfaces or being bacterially mediated [Landing and Bruland, 1987]. Sediment resuspension thus does not lead to a net release of manganese to the dissolved phase, but rather may enhance the removal of manganese due to the production of higher particle concentrations.

The incubation of particles collected at the lower shear stress of $\tau=0.18 \mathrm{Nm}^{-2}$ shows only small releases to the dissolved phase of lead, copper and barium by the end of the experiment. The filtered treatments during the same time remained constant within the error of the analysis, except for the barium concentrations. The $\mathrm{Ba}$ concentration in the filtered treatment shows a rise after the first 3 hours. This rise may be due to barium contamination introduced after the first sampling. At 42 hours there is a sharp release of iron, copper and barium to the dissolved phase. The high concentration does not persist until the next time point. The total release due to this spike in concentration is included in Table 5.7.

The concentration of iron at the final time point is below the initial concentration, suggesting net removal of dissolved iron. The filtered treatment also shows a fall in the iron concentration. The removal from the filtered treatment is likely due to the oxidation of dissolved iron and the coagulation of iron colloids into particles greater than $0.45 \mu \mathrm{m}$. Comparing the two treatments suggest that there is a release of dissolved iron from particles. Silver shows a steady release from particles until at 42 hours, when the concentration of silver starts to decline gradually. There is still a net release of silver after 90 hours, but removal processes, such as scavenging, obviously become more important than the release mechanisms after this time.

The particles from the high shear stress incubation $\tau=0.3 \mathrm{Nm}^{-2}$ show much more significant and consistent release to the dissolved phase of iron, silver and copper. There is a slight decrease in the dissolved concentrations in the filtered treatments of these species over the same time. This decrease may be due to adsorption to the walls of the container or coagulation of colloidal phases. To calculate the total release from the particles in the unfiltered solutions the removal observed in the filtered treatment is subtracted in Table 5.7 
to give total release from particles.

The fraction of each trace metal released to the dissolved phase from resuspended particles after 90 hours is also given in Table 5.7. Between 5-7\% of silver, copper and barium are released from the high shear stress incubation, while less than $1 \%$ of each of these metals is released from the low shear stress incubation. The release of lead and iron is minimal in both incubations, while there is a removal of manganese. The difference in the percentage released between the two shear stress levels may be due to a number of reasons. If smaller particles dominate the size distribution in the low shear stress level, the scavenging may be greater due to higher surface areas. This may act to remove any released material, so giving the impression of a lower release. The persistent release over time does however point to a chemical mechanism driving the release in the higher shear stress incubation. The difference could be due to erosion of material from different redox zones. At the low shear stress, oxidized material is eroded, so there is only minimal release, likely due to ion exchange reactions or oxidation of particles in anoxic micro-environments. The enriched surface particles discussed in Section 5.3.4 are being eroded at a shear stress of $0.18 \mathrm{Nm}^{-2}$. If the enriched layer is due to iron and manganese oxides at the sediment-water interface scavenging trace metals, then release into an oxic water column does not change the redox conditions, so there is no release due to reductive dissolution.

The $0.3 \mathrm{Nm}^{-2}$ incubation have a much greater release because sub-oxic particles are resuspended into the water column at this shear stress. Sediments from deeper within the sediment profile are eroded and it is likely that sulfides exist at this depth. Oxidation of the sulfides will lead to the release of trace metals to the dissolved phase. There is a release of $\mathrm{Fe}, \mathrm{Ag}, \mathrm{Pb}$ and $\mathrm{Cu}$, elements which readily form sulfides and no release of either barium or $\mathrm{Mn}$, neither of which readily form sulfides.

The incubation experiments indicate that for many of the species there is a continued release to the dissolved phase from resuspended particles. As this longer term release is an important source of dissolved species to the overlying waters it is worth considering how these experiments could be improved to provide a more realistic simulation of the processes occurring during a storm. The incubation experiments do not accurately simulate the particle dynamics that would be experienced in-situ after a storm because a constant concentration of suspended solids is maintained. In reality the concentration increases and then drops off. The state of flocculation will change during these changes in suspended 
concentration, and this may have an impact on the dissolved:solids ratio. An incubation experiment with more controlled levels of turbulence and with suspended solids that are allowed to settle could simulate the particle dynamics more accurately. Field measurements during and after a resuspension event, with a high temporal coverage close to the sedimentwater interface would also be invaluable for more accurately quantifying the long-term release from eroded particles.

\subsubsection{Temporal Variation of Shear Stresses}

The erosion chamber experiments provide an estimate of the flux associated with a single resuspension event at a particular shear stress. In order to calculate a total release due to resuspension in Boston Harbor over the course of a year, the frequency of occurrence of each shear stress needs to be determined and combined with the fluxes measured for each shear stress increment. To determine the bottom shear stresses experienced by the Hingham Bay site a combined modeling and statistical approach was used. Dr Rich Signell at the USGS performed all of the analyses to determine the shear stresses. The method used is described here briefly.

Hourly wind data collected by the Boston meteorological buoy number 44013 was available for a 19.4 year time period, August 1984 to December 2003. This buoy is located in Massachusetts Bay, $20.6 \mathrm{~km}$ northeast of the Hingham Bay site. Gaps in the data less than 1 day were filled with interpolated data and gaps greater than one day were filled with data from the same timespan in 1994. Wind stresses were calculated from the formulation of Large and Pond [1981]. Tidal currents for the 19.4 year period were predicted using tidal constituents for the Hingham Bay site. The constituents were determined by running a $100 \mathrm{~m}$ resolution depth-averaged tidal model TRIM [Signell and Butman, 1992] driven by the 5 dominant NOAA tidal constituents for Boston Harbor (O1, K1, N2, M2, S2). The model was run for one year, with the time series output at the Hingham Bay site recorded every 0.1 hours. The tidal constituents for the Hingham Bay site were determined using the program of Pawlowicz et al. [2002] and a 19.4 year period prediction created from the tidal constituents.

The wind driven currents for the 19.4 year period were predicted using a transfer function between wind and current determined for each 10 degrees of wind angle. Winds of magnitude 5,10 and $15 \mathrm{~m} / \mathrm{s}$ were used to verify that the current response is essentially a linear function 
of wind stress. A constant transfer factor for each wind angle was determined, and the input series of wind stress angle and magnitude converted into current angle and magnitude. Wave driven currents for the 19.4 year period were determined by calculating the wave height and period using the shallow water, fetch limited wave formula from the US Army Corps of Engineers Shore Protection Manual. The bottom orbital velocities were calculated using the explicit method detailed in Dean and Dalyrymple [1991].

For waves and currents exceeding $0.01 \mathrm{~m} \mathrm{~s}^{-1}$, the Grant and Madsen [1979] wavecurrent interaction formula was used to calculate combined shear stress. The depth-averaged currents (tide + wind-driven) were converted to $1 \mathrm{~m}$ above bottom using the model drag coefficient $\left(c_{d}=0.003\right)$. A constant bottom roughness of $0.0001 \mathrm{~m}$ was assumed.

The wind speed data from Buoy 44013 is shown in Figure 5-19, and a distinct seasonal pattern is evident with stronger winds occurring in the winter months. The maximum simulated depth-averaged tidal current at the Hingham Bay site was $22 \mathrm{~cm} \mathrm{~s}^{-1}$ and the maximum depth averaged wind driven current was $44 \mathrm{~cm} \mathrm{~s}^{-1}$. The maximum wave bottom orbital velocity was $21 \mathrm{~cm} \mathrm{~s}^{-1}$. The total simulated current at $1 \mathrm{~m}$ above the bottom at the Hingharn Bay site is shown in Figure 5-20 and the corresponding combined wind-current bottom shear stress is shown in Figure $5-21$.

To determine the importance of sediment resuspension to naetal fluxes at Hingham Bay the measured releases in the erosion chamber at each shear stress level are coupled with the simulated shear stress record. The number of times each imposed shear stress level is reached (an event) in the record is shown in Table 5.8. If two events occurred within 24 hours they were defined as only a single event to take into account sediment recovery time. The actual time for an event to be independent of a previous event is unknown, but 24 hours was selected here. Successive erosion experiments performed on the same core but with a resting period in between the experiments could be used to determine a more appropriate time for sediment recovery. 


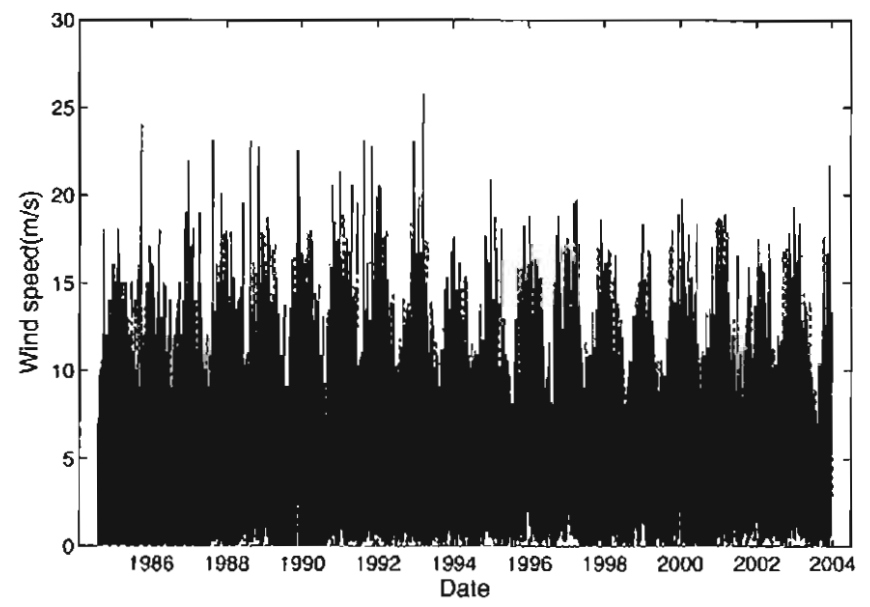

Figure 5-19: Buoy 44013 wind speed data

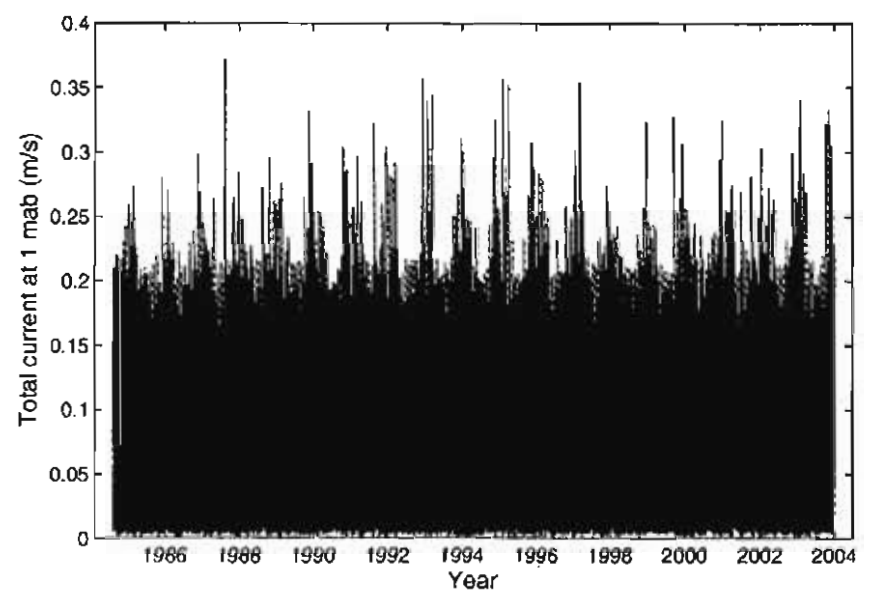

Figure 5-20: Simulated current at $1 \mathrm{~m}$ above the bottom at the Hingham Bay site.

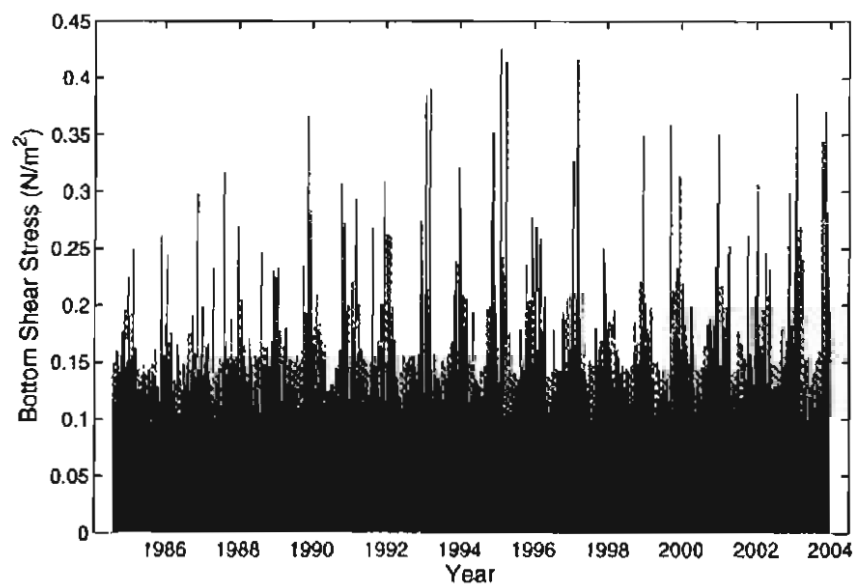

Figure 5-21: Simulated bottom shear stress distribution at the Hingham Bay site. 
Table 5.8: Number of Shear Stress Events in Hingham Bay Siraulated Record

\begin{tabular}{lcc}
\hline $\begin{array}{l}\text { Shear Stress } \\
\left(\mathrm{Nm}^{-2}\right)\end{array}$ & $\begin{array}{c}\text { Number of Events } \\
\text { in Simulated Record }\end{array}$ & $\begin{array}{c}\text { Average Number of Events } \\
\text { (/year) }\end{array}$ \\
\hline 0.12 & 1245 & 64 \\
0.14 & 729 & 36 \\
0.18 & 191 & 9.8 \\
0.21 & 107 & 5.5 \\
0.26 & 46 & 2.4 \\
0.3 & 21 & 1.1 \\
0.35 & 10 & 0.5 \\
\hline
\end{tabular}

From Table 5.8 it can be seen that the erosion threshold at the Hingham Bay site is exceeded 64 times per year. In order to observe the dominant forcing driving the low shear stress occurrences a more detailed two month record of currents and shear stresses is shown in Figure 5-22 for March and April 2000. The erosion threshold is exceeded several times during each spring tide. More intense erosion events are due to strong winds associated with the passage of storms. However, the impact of a wind event is strongly dependent on both wind direction and the tidal stage when the wind event occurs.

\subsubsection{Total Metal and Nutrient Fluxes to Boston Harbor}

The amount of material released at two selected shear stresses are shown in Table 5.9 for metals and Table 5.10 for nutrients. A comparison of these fluxes to other known loadings requires an estimate of the areal extent over which the porewater and resuspension fluxes measured at the two sites can be applied. As an initial estimate of the magnitude of the fluxes the Hingham Bay site is taken to be representative of all the fine grained sediment sites within the Harbor. As only one site has been sampled within this fine-grained area, this is a crude estimate, but it will provide a useful order of magnitude indication of the importance of the benthic fluxes. The depositional sediments in Boston Harbor [Knebel et al., 1991] cover an area of $41 \mathrm{~km}^{2}(33 \%)$ of Boston Harbor and this is the area used to scale up the porewater fluxes to give a Harbor wide estimate of diffusive fluxes. This area is also used to give an estimate of the resuspension fluxes. All the depositional regions within the Harbor will not experience the same shear stress during a particular resuspension event. However the Hingham Bay site is a sheltered site with limited fetch from most wind directions. The water depth is close to the average Harbor depth. Estimates of the 

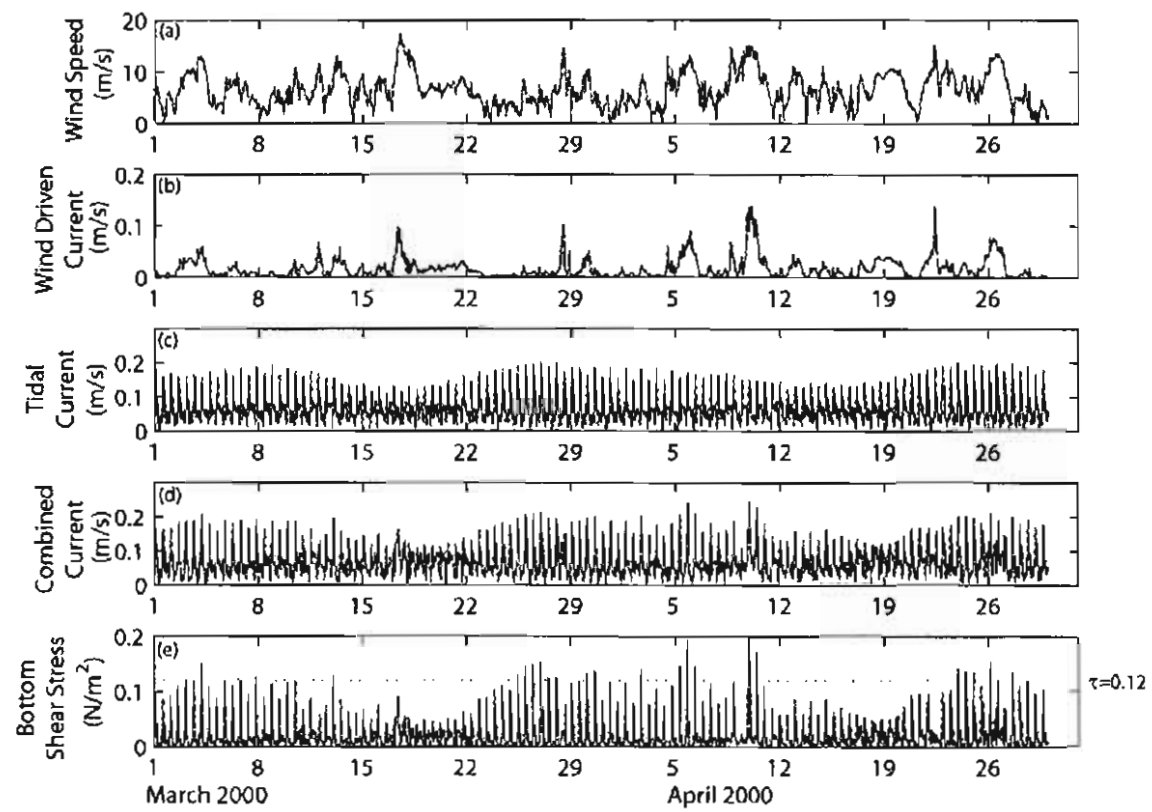

Figure 5-22: Two month time series of data used to calculate the combined wave-current bottom shear stress. (a) buoy 44013 wind speed data, (b) magnitude of depth-averaged wind driven current, (c) magnitude of depth-averaged tidal current (d) combined (tide+wind) current at $1 \mathrm{mab}(\mathrm{e})$ combined wave-current bottom stress.

occurrence of shear stresses at this site are thus likely to be underestimates for the other less sheltered, shallower depositional areas. The erosional fluxes measured at the Hingham Bay site can thus be scaled up to give a lower bound estimate for resuspension fluxes. To improve this estimate additional modeling would be required to determine the distribution of shear stresses during sediment resuspension events across the Harbor. The Massachusetts Bay site is also a depositional site. There are only $7.2 \mathrm{~km}^{2}$ of depositional sites within Massachusetts Bay west of $70^{\circ} 41.3^{\prime} \mathrm{W}$. This area is used to scale up the porewater and sediment resuspension fluxes. The occurrence of shear stresses at the Massachusetts Bay site will be representative of the other depositional areas because all the regions are offshore, so have similar exposure to winds and are found at similar water depths.

The release of material at each shear stress level is combined with the number of simulated events at each shear stress level from Table 5.8 to provide an estimate of the annual average release due to sediment resuspension. The results are shown in Table 5.11.

To determine the importance of sediment resuspension fluxes to the total metal loading in Boston Harbor the known sources of contaminants are also compared in Table 5.11. The 
Table 5.9: Solute and Solid Phase Release for Each Erosion Experiment

\begin{tabular}{|c|c|c|c|c|c|c|c|c|c|c|c|}
\hline \multirow{2}{*}{\multicolumn{2}{|c|}{$\begin{array}{c}\text { Eroded } \\
\text { Depth } \\
(\mathrm{mm})\end{array}$}} & \multicolumn{5}{|c|}{ Solid Flux } & \multicolumn{5}{|c|}{ Solute Flux } \\
\hline & & $\begin{array}{c}\mathrm{Fe} \\
\text { (mmo }\end{array}$ & $\begin{array}{c}\mathrm{Mn} \\
\left.\mathrm{m}^{-2}\right) \\
\end{array}$ & \multicolumn{2}{|c|}{$\left(\mu \mathrm{mmolm}^{-2}\right)$} & $\begin{array}{r}\mathrm{Cu} \\
-2) \\
\end{array}$ & \multicolumn{2}{|c|}{$\left(\mu \mathrm{mol} / \mathrm{m}^{-2}\right)$} & \multicolumn{3}{|c|}{$\left(\mathrm{nmol} / \mathrm{m}^{-2}\right)$} \\
\hline \multicolumn{12}{|c|}{ Hingham Bay October 2003} \\
\hline$\tau=0.18 \mathrm{Nm}^{-2}$ & 0.4 & 95 & 3 & 8 & 84 & 520 & 170 & 29 & 6 & -290 & 2400 \\
\hline$\tau=0.3 \mathrm{Nm}^{-2}$ & 3.5 & 1800 & 29 & 66 & 660 & 2900 & 830 & 180 & 14 & 87 & 2070 \\
\hline \multicolumn{12}{|c|}{ Hingham Bay June 2004} \\
\hline$\tau=0.18 \mathrm{Nm}^{-2}$ & 0.4 & 44 & 0.7 & 7.4 & 83 & 300 & 379 & 101 & 29 & -159 & 2650 \\
\hline$\tau=0.3 \mathrm{Nm}^{-2}$ & 4.8 & 990 & 15 & 54 & 290 & 1900 & 680 & 329 & 25 & -15 & 2360 \\
\hline \multicolumn{12}{|c|}{ Massachusetts Bay September 2003} \\
\hline$\tau=0.18 \mathrm{Nm}^{-2}$ & 0.5 & 0.25 & 0.01 & 3.7 & 77 & 210 & n.m. & 18 & 43 & 330 & 2260 \\
\hline$\tau=0.3 \mathrm{Nm}^{-2}$ & 2.6 & 450 & 9 & 22 & 470 & 930 & n.m. & 45 & 33 & 260 & 2300 \\
\hline
\end{tabular}

Table 5.10: Nutrient Release for Each Hingham Bay Erosion Experiment

\begin{tabular}{lcccc} 
& Ammonium & $\begin{array}{c}\text { Phosphate } \\
\left(\mu \text { molm }^{-2}\right)\end{array}$ & Sitrate & Silicic Acid \\
\cline { 1 - 3 } Hingham Bay October 2003 & & & \\
$\tau=0.18 \mathrm{Nm}^{-2}$ & 260 & 150 & 540 & 650 \\
$\tau=0.3 \mathrm{Nm}^{-2}$ & 910 & 960 & 760 & 3500 \\
Hingham Bay June 2004 & & & \\
$\tau=0.18 \mathrm{Nm}^{-2}$ & 860 & 150 & 0 & 2400 \\
$\tau=0.3 \mathrm{Nm}^{-2}$ & 1000 & 310 & 0 & 6300 \\
\hline
\end{tabular}


Table 5.11: Summary of Fluxes to Boston Harbor

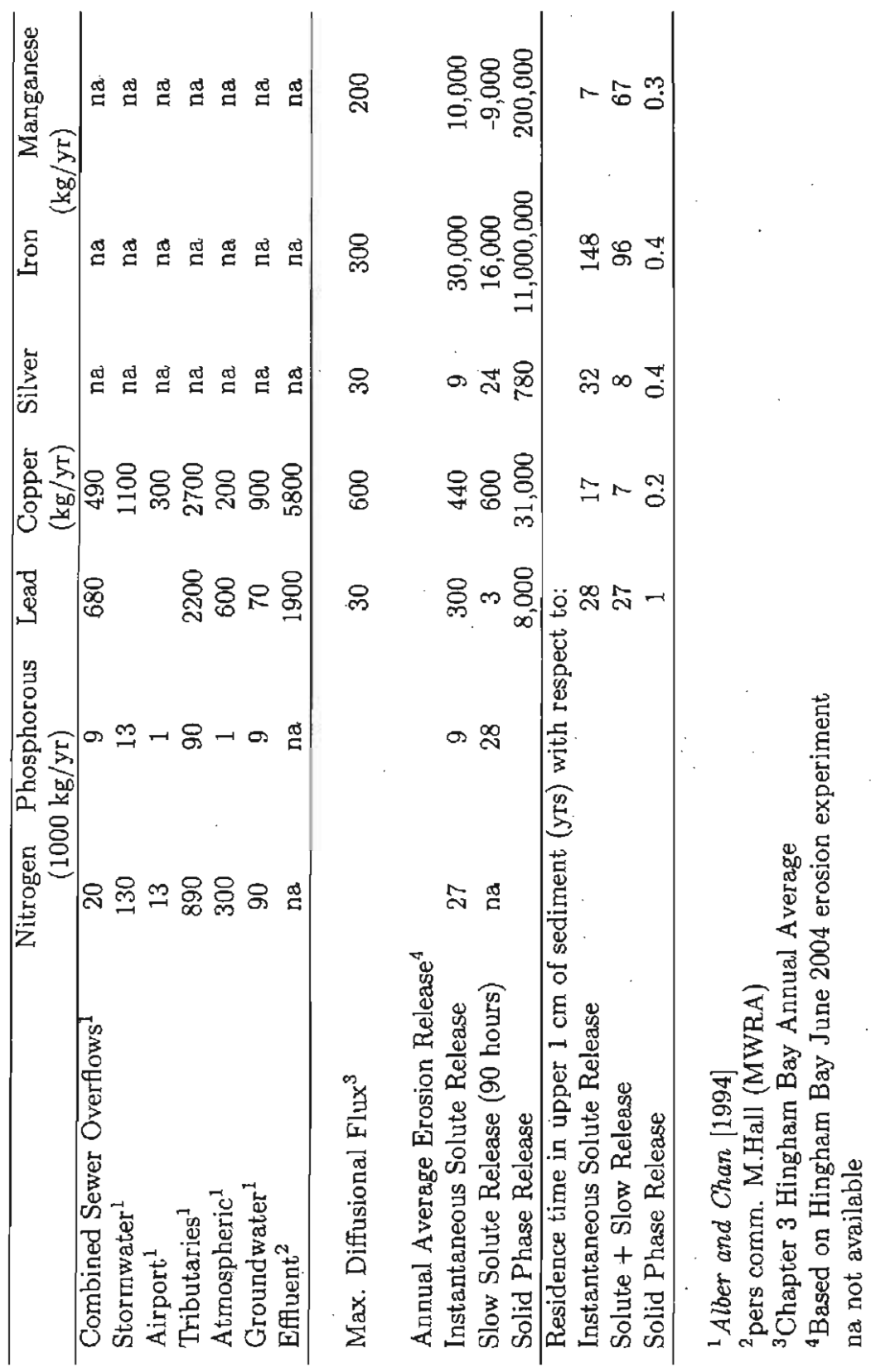


inputs due to rivers, atmospheric deposition, groundwater and combined sewer overflows (CSOs) were calculated from sampling data from 1990 - June 1993 by Alber and Chan [1994]. This is the most recent source data available for Boston Harbor, despite the fact that continuing source reduction is underway. For example of the $84 \mathrm{CSO}$ modeled in the study by Alber and Chan [1994], 21 have since been closed, so that the CSO inputs given are now an overestimate. Due to the closure of the Nut Island treatment plant in 1991, the addition of secondary treatment to the Deer Island treatment facility over 1995 to 2000 and the relocation of sewage discharge from the mouth of Boston Harbor to $15.3 \mathrm{~km}$ east in Massachusetts Bay (http : //www.mwra.state.ma.us/03sewer/html/sewcso.htmand/sewditp.htm) the discharge of metals in effluent and sludge to Boston Harbor is now reduced by almost $90 \%$ compared to discharges in the 1980's (data for 1989 - 2002 courtesy M.Hall Massachusetts Water Resources Authority). Despite these significant improvements, the load of copper discharged in effluent to Massachusetts Bay is still the largest source of copper to the Bay compared to other water and atmospheric inputs. The discharge of lead in effluent is now comparable to the lead that reaches the Bay from rivers.

The diffusive fluxes for each metal calculated from the porewater profiles of Chapter 3 are also provided in Table 5.11. The diffusive flux of copper in Boston Harbor is of the same order of magnitude as atmospheric, groundwater and CSO fluxes, and are an order of magnitude lower than the input due to effluent and tributaries. The sediments may actually remove lead from the water due to diffusion into the sediments.

The solid phase resuspension release of lead and copper is greater than all the other inputs combined. One resuspension event of $0.18 \mathrm{Nm}^{-2}$ releases a quantity of metals in the solid phase of comparable magnitude to the input from effluent and tributaries and a single shear stress event of $0.3 \mathrm{Nm}^{-2}$ mobilizes copper and lead in comparable amounts to what is discharged as effluent over an entire year. The release of solid phase material to the water column is less ecologically important than the release of dissolved material due to much lower bioavailability of the metal and because a large proportion of the suspended material will settle back to the sediments. However that such large quantities of metals are mobilized on an annual basis is significant for determining the long-term re-distribution of contaminated sediments.

The dissolved phase releases due to resuspension of iron and manganese are at least 50 times higher than the calculated diffusive benthic fluxes. This is because resuspension short 
circuits the oxidative trapping of these metals at the sediment-water interface. The removal of the released Mn onto resuspended particles largely counteracts this Mn release and will limit the long distance dispersal of $\mathrm{Mn}$ in the dissolved phase. For silver, release to the dissolved phase from resuspended particles over time is the most important term associated with resuspension and is the same order of magnitude as the calculated benthic fluxes.

The combined solute release due to benthic processes (=benthic flux + erosion release) is significant and of the same order of magnitude as the river input of copper to the Harbor. and about $25 \%$ of the discharge due to effluent. The dissolved flux of lead from erosion is of similar magnitude to the atmospheric deposition and is $10 \%$ of the loadings from effluent and tributaries. The effluent source term given in Table 5.11 is that which is discharged to Massachusetts Bay. Due to the considerably better dilution factors from the new, offshore outfall [Signell et al., 2000] the effluent term is no longer a significant source of metals to Boston Harbor. Considering only the metal sources to Boston Harbor then gives that the benthic flux of copper and lead are $60 \%$ and $15 \%$ of the riverine input and $30 \%$ and $8 \%$ of the total loadings from all other sources. The calculated fluxes from all other sources is for total metal (dissolved + particulate), while the benthic fluxes considered in this calculation are for the dissolved phase only, thus further increasing the importance of benthic fluxes to the metal budget of Boston Harbor. The sediments are thus a very significant source of dissolved copper to the Harbor. Although numerically the erosional release of dissolved lead is not as significant, it is important for the long-term distribution of lead as due to strong lead scavenging by particles, benthic processes act largely to remove lead from the water column and sediment resuspension at high shear stresses is the only way lead can be remobilized from sediments to the dissolved phase. The importance of both diffusive and resuspension fluxes of metals to Boston Harbor will increase as continuing source reduction reduces the contaminants discharged from effluent, tributaries and CSOs.

The residence time of a metal in the sediment with respect to various fluxes can be determined by

$$
\text { Residence Time }=\frac{\text { Total Inventory of Metals }}{\text { Flux } \times \text { Area }}
$$

Considering only the upper $1 \mathrm{~cm}$ of sediment for the total metal inventory and using porosity and solid phase data from Tables 5.2 and 5.6 the residence times are given in Table 5.11. This analysis does not consider additional processes such as bioturbation or continuing metal 
fluxes to the sediment, so does not represent the actual residence time of a metal in the sediments. The residence time as calculated here is only useful for assessing the importance of sediment resuspension relative to the concentrations of metals currently in the surface sediments. The residence times for all metals with respect to solid phase fluxes are all less than $1 \mathrm{yr}$. As the metal concentrations in the sediments have not been observed to fall within such a rapid timescale this suggests that a considerable fraction of the resuspended solid phase is redeposited close to its point of origin. Large resuspension and transport events will be very important for redistributing contaminant metals, but from a bioavailability point of view it is more important to consider release to the solute phase.

The residence times of silver, lead and copper with respect to solute release due to resuspension are between 20-30 years. This timescale suggests that erosional release needs to be considered in long-term management plans of these contaminated sediments. The fluxes are sufficiently high to be significant terms in the metal loadings to Boston Harbor, but are also low enough so that erosional release will be a persistent source of these metals for many decades.

\subsubsection{Conclusions}

The release of metals and nutrients due to the resuspension of sediments at two coastal sites in Massachusetts has been investigated to determine the importance of resuspension in mobilizing these species from contaminated sediments. A laboratory-based erosion chamber was used to determine the release of material over the range of shear stresses encountered at each site. Significant resuspension did not occur at either site until critical shear stresses of between $0.12-0.14 \mathrm{Nm}^{-2}$ were exceeded. From hydrodynamic modeling it was determined that at the contaminated site in Boston Harbor, this condition was met every spring tide. With shear stresses increasing above the critical shear stress the quantity of solids resuspended increased. The maximum shear stress imposed in the chamber was $0.3 \mathrm{Nm}^{-2}$, a level reached on average once per year at the Harbor site. At this high shear stress sediment was found to erode to a maximum depth of only $5 \mathrm{~mm}$ at each site. Despite this small depth of erosion the quantity of solid phase lead and copper mobilized into the water column over a year due to resuspension is greater than the total release of these metals due to effluent, river and groundwater discharge combined.

The concentration of metals in the particles that are resuspended first are enriched 
relative to the concentration in the bulk surface sediments. The importance of shear stresses at or just above the erosion threshold in remobilizing metals is thus enhanced as the highest concentrations of metals are eroded at shear stresses that occur with greater than a monthly frequency. The fine material that remains in suspension after 8 hours is also found to be enriched in metals above the bulk sediment concentration. This material remains in suspension over timescales that allow transportation of contaminated particles beyond the site of mobilization. More detailed investigations of metal concentrations in settling particles together with settling velocity analysis is required to quantify the importance fine grained sediment transport in contaminant redistribution.

The release of solutes due to resuspension although 2 to 3 orders of magnitude smaller than the solid fluxes are important due to the greater bioavailability and mobility of dissolved species. Two different categories of solute release during a resuspension event were quantified. The first is the release that occurs rapidly as the sediments are resuspended. Silver, lead, copper and iron as well as the nutrient species phosphate, total inorganic nitrogen and silicic acid are all released as solutes in greater amounts than porewater flushing alone would contribute. Advection within the sediments that extends deeper than the depth of erosion may contribute to this enhanced release but comparison of the ratios of species found in porewaters and in the erosion chamber indicates that chemical mechanisms are also responsible for the enhanced release. Ion-exchange reactions of species adsorbed to resuspended particles can account for all the phosphate and ammonium released.

Release from particles suspended in the water column for over an hour is the second mode of solute release and was quantified by incubating resuspended sediments in oxygenated water for up to 90 hours. The sediment eroded at a shear stress of $0.3 \mathrm{Nm}^{-2}$ released more metals, silicic acid and phosphate from particles than sediment eroded at a stress of 0.18 $\mathrm{Nm}^{-2}$. Sediments from deeper within the sediment profile are eroded at the higher shear stress, so release due to oxidation of reduced species is greater. $5 \%$ of the silver and $6 \%$ of the copper associated with particles was released into the dissolved phase over the 90 hour incubations. Manganese was removed from solution and only $0.3 \%$ of iron was transferred to the dissolved phase.

In Boston Harbor the quantity of lead and copper released to the solute phase by sediment resuspension each year is about $8 \%$ of the combined loadings of these metals from atmospheric deposition, riverine, stormwater and groundwater discharge to Boston Harbor. 
If the longer term release from suspended particles as well as diffusive benthic fluxes are also considered, benthic processes contribute solutes that are $30 \%$ of the total known discharges of copper and $9 \%$ for lead. Benthic release will become even more significant as continued pollutant source reduction occurs and the discharge of metals from municipal facilities is decreased. This study is the first quantification of metal fluxes due to resuspension and although not the largest source of metals to the waters of coastal Massachusetts, the values determined are significant and will become increasingly important terms in the metal budgets of coastal waters and so should be considered in the long-term management of contaminated sediments.

These resuspension fluxes are the first quantitative values to be determined, further experiments could be undertaken to improve the estimates. Additional sampling sites within the fine-grained sediment region of Boston Harbor would provide an indication of the variability between sites and improve the Harbor-wide flux calculation. Many of the resuspension events that exceed the critical erosion threshold occur over a spring tide, spaced at intervals of just over 24 hours. Investigations focussing on how quickly a sediment recovers from resuspension events would improve estimates, especially improving the estimates of the contributions from the more frequent lower shear stress events. The incubation experiments indicate that for many of the species there is a continued release to the dissolved phase from resuspended particles. More accurate simulation of the particle dynamics during a storm, to allow investigation of the roles of flocculation and sedimentation, will provide a more realistic estimate of the release from eroded particles. Finally field investigations during resuspension events will be invaluable in both verifying and improving the fluxes determined using the erosion chamber. As the erosion chamber investigations have indicated that resuspension is an important source of dissolved metals to the waters of Boston Harbor, these additional experiments are necessary for furthering our understanding of the fate of metal in contaminated sediments. 


\section{References}

Adams, N., and J. Kramer, Reactivity of $\mathrm{Ag}^{+}$ion with thiol ligands in the presence of iron sulfide, Environmental Toxicology and Chemistry, 17(4), 625-629, 1998.

Ahner, B., and F. Morel, Phytochelatin production in marine algae. 2. Induction by various metals, Limnology and Oceanography, 40(4), 658-665, 1995.

Ahner, B., , and S. K. Morel, Phytochelatin production in marine algae. 2. An interspecies comparison, Limnology and Oceanography, 40(4), 649-657, 1995.

Ahner, B., L. Wei, J. Oleson, and N. Ogura, Glutathione and other low molecular weight thlols in marine phytoplankton under metal stress, Marine Ecology-Progress Series, 292, 93-103, 2002.

Al-Farawati, R., and C. V. D. Berg, Thiols in coastal waters of the western North Sea and English Channel, Environmental Science and Technology, 35(10), 1902-1911, 2001.

Al-Farawati, R., and C. van den Berg, Metal-sulfide complexation in seawater, Marine Chemistry, 63(3-4), 331-352, 1999.

Alber, M., and A. Chan, Sources of contaminants to Boston Harbor: revised loading estimates, Tech. rep., Massachusetts Water Resources Authority, 1994.

Aller, R., Diagenetic processes near the sediment-water interface of Long Island Sound. II Fe and $\mathrm{Mn}$, Advances in Geophysics, 22, 351-415, 1980.

Aller, R., The sedimentary Mn cycle in Long Island Sound: its role as intermediate oxidant and the influence of bioturbation, $\mathrm{O} 2$, and Corg flux on diagenetic reaction balances, Journal of Marine Research, 52, 259-295, 1994.

Amos, C., G. Daborn, H. Christian, A. Atkinson, and A. Robertson, In-situ erosion measurements on fine-grained sediments from the Bay of Fundy, Marine Geology, 108(2), 175-196, 1992a.

Amos, C., J. Grant, G. Daborn, and K. Black, Sea Carousel - a benthic, annular flume, Estuarine Coastal and Shelf Science, 34(6), 557-577, 1992 b.

Andersen, T., K. Jensen, L. Lund-Hansen, K. Mouritsen, and M. Pejrup, Enhanced erodibility of fine-grained marine sediments by hydrobia ulvae, Joumal of Sea Research, 48(1), 51-58, 2002.

Andrews, D., and A. Bennett, Measurements of diffusivity near the sediment-water interface with a fine-scale resistivity probe, Geochimica et Cosmochimica Acta, 45, 2169-2175, 1981.

Ankley, G., D. D. Toro, D. Hansen, and W. Berry, Assessing the ecological risk of metals in sediments, Environmental Toxicology and Chemistry, 15(12), 2053-2055, 1996.

Balistrieri, L., P. Brewer, and M. J.W., The adsorption of $\mathrm{Cu}, \mathrm{Pb}, \mathrm{Zn}$ and $\mathrm{Cd}$ on goethite from major ions in seawater, Geochimica et Cosmochimica Acia, 46, 1253-1267, 1982.

Benoit, G., and $\mathrm{H}$. Hemond, $210 \mathrm{Po}$ and $210 \mathrm{~Pb}$ remobilization from lake sediments in relation to iron and manganese cycling, Environmental Science and Technology, 24, 1224-1234, 1990.

Berner, R., Early Diagenesis, Princeton University Press, Princeton, N.J., 1980.

Boning, P., H. Brumsack, M. Bottcher, B. Schnetger, C. Kriete, J. Kallmeyer, and S. Borchers, Geochemistry of Peruvian near-surface sediments, Geochimica et Cosmochimica Acta, 68(21), 44294451, 2004.

Bothner, M., M. B. ten Brink, and F. Manheim, Metal concentrations in surface sediments of Boston Harbor - changes with time, Marine Environmental Research, 45(2), 127-155, 1998. 
Bothner, M., M. Casso, R. Rendigs, and P. Lamothe, The effect of the new Massachusetts Bay sewage outfall on the concentrations of metals and bacterial spores in nearby bottom and suspended sediments, Marine Pollution Bulletin, 44(10), 1063-1070, 2002.

Boulegue, J., Trace metal ( $\mathrm{Fe}, \mathrm{Cu}, \mathrm{Zn}, \mathrm{Cd}$ ) in anoxic environments, in Trace Metals in Seawater, Marine Science Series, vol. 9, edited by C. Wong, E. Boyle, K. Bruland, J. Burton, and E. Goldberg, pp. 563-577, NATO, 1983.

Boulegue, J., C. Lord, and T. Church, Sulfur speciation and associated trace metals $(\mathrm{Fe}, \mathrm{Cu})$ in the pore waters of Great Marsh, Delaware, Geochimica et Cosmochimica Acta, 46, 453-464, 1982.

Boyle, E., and J. Edmond, Determination of copper, nickel, and cadmium in sea water by APDC chelate coprecipitaion and flameless atomic absorption spectrometry, Analytica Chimica Acta, 91, 189-197, 1977.

Brendel, P., and G. Luther, Development of a gold amalgam voltametric microelectrod for the determination of dissolved $\mathrm{Fe}, \mathrm{Mn}, \mathrm{O} 2$ and $\mathrm{S}(-\mathrm{II})$ in porewaters of marine and freshwater sediments., Environmentol Science and Technology, 29, 751-761, 1995.

Briggs, P., and A. Meier, The determination of forty-two elements in geological materials by inductively coupled mass spectrometry, Open File Report 99-166, U.S. Geological Survey, 1999.

Bruland, K., Trace elements in seawater, in Chemical Oceanography, vol. 8, 2nd edition ed., pp. 147220, Academic, London, 1983.

Buchman, M., NOAA screening quick reference tables, NOAA HAZMAT Report 99-1, Coastal Protection and Restoration Division, NOAA, 1999.

Burdige, D., The biogeochemistry of manganese and iron reduction in marine sediments, Earth-Science Reviews, 35, 249-284, 1993.

Burdige, D., and K. Gardner, Molecular weight distribution of dissolved organic carbon in marine sediment pore waters, Marine Chemistry, 62(1-2), 45-64, 1998.

Cai, W.-J., Y. Wang, and R. Hodson, Acid-base properties of dissolved organic matter in the estuarine waters of Georgia, Geochimica et Cosmochimica Acta, 62, 473-483, 1998.

Calvo, C., R. N. Donazzolo, F. Guidi, and A. Orio, Heavy metal pollution studies by resuspension experiments in Venice Lagoon, Water Research, 25(10), 1295-1302, 1991.

Canfield, D., Reactive iron in marine sediments, Geochimica et Cosmochimica Acta, 53, 619-632, 1989.

Canfield, D., B. Thamdrup, and J. Hansen, The anaerobic degradation of organic matter in Danish coastal sediments: Iron reduction, manganese reduction and sulfate reduction, Geochimica et Cosmochimica Acta, 57, 3867-3883, 1993.

Cantwell, M., R. Burgess, and D. Kester, Release and phase partitioning of metals from anoxic estuarine sediments during periods of simulated resuspension, Environmental Science and Technology, $36(24), 5328-5334,2002$.

Chen, K., and S. Gupta, Formations of polysulfides in aqueous solution, Environmental Letters, $4(3)$, $187-200,1973$.

Ciceri, G., C. Maran, W. Martinotti, and G. Queirazza, Geochemical cycling of heavy metals in a marine coastal area: benthic flux determination from pore water profiles and in situ measurements using benthic chambers, Hydrobiologia, 235-236(1), 501-517, 1992.

Cline, J., Spectrophotometric determination of hydrogen sulfide in natural waters, Limnology and Oceanography, 14(3), 454-458, 1969.

Cooper, D., and J. Morse, Biogeochemical controls on trace metal cycling in anoxic marine sediments, Environmental Science and Technology, 32(3), 327-330, 1998.

Crusius, J., and J. Thomson, Mobility of authigenic rhenium, si]ver, and selenium during postdepositional oxidation in marine sediments, Geochimica et Cosmochimica Acta, 67(2), 265-273, 2003.

Crusius, J., M. Bothner, and C. Sommerfield, Bioturbation depths, rates and processes in Massachusetts Bay sediments inferred from modeling of $210 \mathrm{~Pb}$ and $239+240 \mathrm{Pu}$ profiles, Estuarine, Coastal and Shelf Science, 61, 243-255, 2004.

Davis, J., and J. Leckie, Effect of adsorbed complexing ligands on trace metal uptake by hydrous oxides, Environmental Science and Technology, 12, 1309-1315, 1978. 
Davison, W., G. Grime, J. Morgan, and K. Clarke, Distribution of dissolved iron in sediment pore waters at submillimetre resolution, Nature, 952, 323-325, 1991.

Davison, W., H. Zhang, and G. Grime, Performance characteristics of gel probes used for measuring the chemistry of pore waters, Environmental Science and Technology, 28, 1623-1632, 1994.

Davison, W., G. Fones, and G. Grime, Dissolved metals in surface sediment and a microbial mat at 100- $\mu \mathrm{m}$ resolution, Nature, 387(6636), 885-888, 1997.

Dean, R., and R. Dalyrymple, Water wave mechanics for engineers and scientists, World Scientific, Teaneck, NJ, 1991.

Diemer, J., C. Quetel, and P. Taylor, Comparison of the performance of different ICP-MS instruments on the measurement of $\mathrm{Cu}$ in a water sample by ICP-IDMS, Journal of Analytical A tomic Spectrometry, $17(9), 1137-1142,2002$.

Donat, J., and K. Bruland, Trace elements in the ocean, in Trace Elements in Natural Waters, edited by B. Salbu and E. Steinnes, pp. 247-281, CRC Press, 1995.

Dupont, C., R. Nelson, S. Bashir, J. Moffett, and B. Ahner, Novel copper-binding and nitrogen-rich thiols produced and exuded by emiliania huxleyi, Limnology and Oceanography, 49(5), 1754-1762, 2004.

Dupont, C., B. Bidigare, J. Moffett, and B. Ahner, Dissolved and particulate biogenic thiols in the North Pacific, In Prep, 2005.

Dyer, K., Coastal and Estuarine Sediment Dynamics, John Wiley and Sons Ltd, Chichester, 1986.

Elderfield, H., R. McCaffrey, N. Luedtke, M. Bender, and V. Truesdale, Chemical diagenesis in Narragansett Bay sediments, American Joumal of Science, 281, 1021-1055, 1981.

Fahey, R., and G. Newton, Determination of low molecular weight thiols using monobromobimane fluorescent labeling and high-perfomance liquid chromatography, Methods in Enzymology, 143, 85$101,1987$.

Field, M., and R. Sherrell, Direct determination of ultra-trace levels of metals in fresh water using desolvating micronebulization and HR-ICP-MS: application to Lake Superior waters, Journal of Analytical Atomic Spectrometry, 18(3), 254-259, 2003.

Field, M., J. Cullen, and R. Sherrell, Direct determination of 10 trace metals in $50 \mu$ l samples of coastal seawater using desolvating micronebulization sector field ICP-MS, Journal of Analytical Atomic Spectroscopy, 14, 1425-1431, 1999.

Flegal, A., S. Sanudo-Wilhelmy, G. Scelfo, P. Yeats, and A. Shiller, Silver in the eastern Atlantic Ocean IOC contaminants baseline study, Marine Chemistry, 49(4), 315-320, 1995.

Fones, G., W. Davison, and G. Grime, Development of constrained DET for measurements of dissolved iron in surface sediments at sub-mm resolution, Science of the Total Environment, 221 (2-3), 127-137, 1998.

Fones, G., W. Davison, O. Holby, B. Jorgensen, and B. Thamdrup, High-resolution metal gradients measured by in situ DGT/DET deployment in Black Sea sediments using an autonomous benthic lander, Limnology and Oceanography, 46(4), 982-988, 2001.

Fones, G., W. Davison, and J. Hamilton-Taylor, The fine-scale remobilization of metals in the surface sediments of the North-East Atlantic, Continental Shelf Research, 24, 1485-1504, 2004.

Froelich, P., et al., Early oxidation of organic-matter in pelagic sediments of the Eastern Equatorial Atlantic - suboxic diagenesis, Geochimica et Cosmochimica Acta, 43(7), 1075-1090, 1979.

Gee, A., and K. Bruland, Tracing $\mathrm{Ni}, \mathrm{Cu}$, and $\mathrm{Zn}$ kinetics and equilibrium partitioning between dissolved and particulate phases in south San Francisco bay, California, using stable isotopes and high-resolution inductively coupled plasma mass spectrometry, Geochimica et Cosmochimica Acta, 66(17), 3063-3083, 2002.

Gerringa, L., Aerobic degradation of organic-matter and the mobility of $\mathrm{Cu}, \mathrm{Cd}, \mathrm{Ni}, \mathrm{Pb}, \mathrm{Zn}$; $\mathrm{Fe}$ and $\mathrm{Mn}$ in marine sediment slurries, Marine Chemistry, 29(4), 355-374, 1990.

Gieskes, J., Interstitial water studies, leg 15: Alkalinity, $\mathrm{pH}, \mathrm{Mg}, \mathrm{Ca}, \mathrm{Si}, \mathrm{PO} 4$ and $\mathrm{NH} 4$, in. Initial Reports of the Deep Sea Drilling Project, edited by B. C. Heezen, chap. Leg 15, pp. 803-829, U.S. Government Printing Office, 1973. 
Giggenbach, W., Optical spectra and equilibrium distribution of polysulfide ions in aqueous solution at $20^{\circ} \mathrm{c}$, Inorganic Chemistry, 11, 1201-1207, 1972.

Giggenbach, W., Kinetics of the polysulfide-thiosulfate disproportionation up to $240^{\circ} \mathrm{c}$, Inorganic Chemistry, 13, 1730-1733, 1974.

Glibert, P., and T. C. Loder, Automated analysis of nutrients in seawater: a manual of techniques, Tech. Rep. WHOI-77-47, Woods Hole Oceanographic Institution, 1977.

Gobeil, C., R. Macdonald, and B. Sundby, Diagenetic separation of cadmium and manganese in suboxic continental margin sediments, Geochimica et Cosmochimica Acta, 61 (21), 4647-4654, 1997.

Gobeil, C., B. Rondeau, and L. Beaudin, Contribution of municipal effluents to metal fluxes in the St. Lawrence River, Environmental Science and Technology, 39, 456-464, 2005.

Grant, W., and $\mathrm{O}$. Madsen, Combined wave and current interaction with a rough bottom, Joumal of Geophysical Research, 84(C4), 1797-1808, 1979.

Guilherme, L., and S. Anderson, Copper sorption kinetics and sorption hysteresis in two oxide-rich soilds (oxisols), in Adsorption of Metals by Geomedia, edited by E. Jenne, pp. 209-228, Academic Press, 1998.

Guo, L., P. Santschi, and K. Warnken, Trace metal composition of colloidal organic material in marine environments, Marine Chemistry, 70(4), 257-275, 2000.

Gust, G., and V. Muller, Interfacial hydrodynamics and entrainment functions of currently used erosion devices, in Cohesive Sediments, edited by N. Burt, R. Parker, and J. Watts, pp. 149-174, Wiley, New York, 1997.

Gwiazda, R., D. Woolard, and D. Smith, Improved lead isotope ratio measurements in environmental and biological samples with a double focussing magnetic sector inductively coupled plasma mass spectrometer (ICP-MS), Journal of Analytical Atomic Spectrometry, 13, 1233-1238, 1998.

Hatje, V., T. Payne, D. Hill, G. McOrist, G. Birch, and R. Szymczak, Kinetics of trace element uptake and release by particles in estuarine waters: effects of $\mathrm{pH}$, salinity, and particle loading, Environment International, 29(5), 619-629, 2003.

Hegarty, S., and T. Villareal, Effects of light level and N:P supply ratio on the competition between phaeocystis cf. pouchetii (hariot) lagerheim (prymnesiophyceae) and five diatom species, Journal of Experimental Marine Biology and Ecology, 226(2), 241-258, 1998.

Heggie, D., D. Kahn, and K. Fischer, Trace metals in metalliferous sediments, MANOP site M: interfacial pore water profiles, Earth and Planetary Science Letters, 80(1-2), 106-116, 1986.

Herrin, R., A. Andren, M. Shafer, and D. Armstrong, Determination of silver speciation in natural waters. 2. Binding strength of silver ligands in surface freshwaters, Environmental Science and Technology, 95(10), 1959-1966, 2001.

Heumann, K., Isotope dilution mass spectrometry of inorganic and organic substances, Fresenius $Z$ Anal Chem, 325, 661-666, 1986.

Heumann, K., Isotope dilution mass spectroscopy, in Inorganic Mass Spectroscopy, edited by F. Adams, pp. 301-376, Wiley, 1998.

Hulanicki, A., Complexation reactions of dithiocarbamates, Talanta, 14, 1371-1392, 1967.

Jacobs, L., and S. Emerson, Trace metal solubility in an anaxic fjord, Earth and Planetary Science Letters, 60, 237-252, 1982.

Jenne, E. (Ed.), Trace Inorganics in Water, Advances in Chemistry Series, vol. 73, American Chemical Society, 1968.

Jocelyn, P., Biochemistry of the SH Group, Academic Press Inc, London, 1972.

Kamyshny, J., A. Goifman, D. Rizkov, and O. Lev, Formation of carbonyl sulfide by the reaction of carbon monoxide and inorganic polysulfides, Environmental Science and Technology, 37, 1865-1872, 2003.

Keller, A., C. Taylor, C. Oviatt, T. Dorrington, G. Holcombe, and L. Reed, Phytoplanktan production patterns in Massachusetts Bay and the absence of the 1998 winter-spring bloom, Marine Biology, 138(5), 1051-1062, 2001.

Kiene, R., Evidence for the biological turnover of thiols in anoxic marine sediments, Biogeochemistry, $13,117-135,1991$. 
Kiene, R., and B. Taylor, Biotransformations of organosulphur compounds in sediments via 3mercaptopropionate, Nature, 392, 148-150, 1988.

Kim, E., R. Mason, E. Porter, and H. Soulen, The effect of resuspension on the fate of total mercury and methyl mercury in a shallow estuarine ecosystem: a mesocosm study, Marine Chemistry, 86(3-4), $121-137,2004$.

Klinkhammer, G., Early diagenesis in sediments from the eastern equatorial Pacific, II. pore water metal results, Earth and Planetary Science Letters, 49(1), 81-101, 1980.

Klinkhammer, G., D. Heggie, and D. Graham, Metal diagenesis in oxic marine sediments, Earth and Planetary Science Letters, 61, 211-219, 1982.

Knebel, H., R. Rendigs, and M. Bothner, Modern sedimentary environments in Boston Harbor, Massachusetts, Journal of Sedimentary Petrology, 61 (5), 791-804, 1991.

Kogut, M., and B. Voelker, Strong copper-binding behavior of terrestrial humic substances in seawater, Environmental Science and Technology, 35(6), 1149-1156, 2001.

Kogut, M., and B. Voelker, Kinetically inert copper in coastal waters, Environmental Science and Technology, 37, 509-518, 2003.

Koschinsky, A., B. Gaye-Haake, C. Arndt, G. Maue, A. Spitzy, A. Winkler, and P. Halbach, Experiments on the influence of sediment disturbance on the biogeochemistry of the deep sea environment, Deep-Sea Research II, 48, 3629-3651, 2001.

Kosower, N., and E. Kosower, Thiol labeling with bromobimanes, Methods in Enzymology, 143, 76-84, 1987.

Kramer, J., et al., Environmental chemistry of silver, in Silver in the Environment: Transport, Fate and Effects, edited by A. Andren and T. Bober, pp. 1-20, SETAC Press, 2002.

Krom, M., and R. Berner, Adsorption of phosphate in anoxic marine sediments, Limnology and Oceanography, 25(5), 797-806, 1980.

Laima, M., H. Matthiesen, L. Lund-Hansen, and C. Christiansen, Resuspension studies in cylindrical microcosms: Effects of stirring velocity on the dynamics of redox sensitive elements in a coastal sediment, Biogeochemistry, 43(3), 293-309, 1998.

Landing, W., and K. Bruland, The contrasting biogeochemistry of iron and manganese in the Pacific Ocean, Geochimica et Cosmochimica Acta, 51, 29-43, 1987.

Large, W., and S. Pond, Open ocean momentum flux measurements in moderate to strong wind, Journal of Physical Oceanography, 11; 324-336, 1981.

Latimer, J., W. Davis, and D. Keith, Mobilization of PAHs and PCBs from in-place contaminated marine resuspension events, Estuarine Coastal and Shelf Science, 49(4), 577-595, 1999.

Lee, J., Contaminated sediment transport in Boston Harbor, Masters, MIT, 1991.

Li, Y., and S. Gregory, Diffusion of ions in seawater and in deep sea sediments, Geochimica et Casmochimica Acta, 38, 703-714, 1974.

Luther, G., A. Giblin, and R. Varsolona, Polarographic analysis of sulfur species in marine porewaters, Limnology and Oceanography, 30(4), 727-736, 1985.

Luther, G., T. Church, J. Scudlark, and M. Cosman, Inorganic and organic sulfur cycling in salt-marsh pore waters, Science, 232, 746-749, 1986.

Luther, G., P. Brendel, B. Lewis, B. Sundby, L. Lefrancois, N. Silverberg, and D. Nuzzio, Simultaneous measurement of $\mathrm{O} 2, \mathrm{Mn}, \mathrm{Fe}, \mathrm{I}-$, and $\mathrm{S}(-\mathrm{II})$ in marine pore waters with a solid-state voltammetric microelectrode, Limnology and Oceanography, 43(2), 325-333, 1998.

Luther, G., B. Glazer, L.Hohmann, J. Popp, M. Tallefert, T. Rozan, P: Brendel, S. Theberge, and D. Nuzzio, Sulfur speciation monitored in situ with solid state gold amalgamvoltammetric microelectrodes: polysulfdes as a special case in sediments, microbial mats and hydrothermal vent waters, Journal of Environmental Monitoring, 3, 61-66, 2001.

Luther, G., S. Theberge, T. Rozan, D. Rickard, C. Rowlands, and A. Oldroyd, Aqueous copper sulfide clusters as intermediates during copper sulfide formation, Environmental Science and Technology, 36 , $394-402,2002$. 
Lyons, W., and W. Fitzgerald, Trace metal speciation in nearshore anoxic and suboxic porewaters, in Trace Metals in Seawater, Marine Science Series, vol. 9, edited by C. Wong, E. Boyle, K. Bruland, J. Burton, and E. Goldberg, NATO, 1983.

Maa, J.-Y., L. Sanford, and J. Halka, Sediment resuspension characteristics in Baltimore. Harbor, Maryland, Marine Geology, 146(1-4), 137-145, 1998.

MacCrehan, W., and D. Shea, Temporal relationship of thiols to inorganic sulfur compounds in anoxic Chesepeake Bay sediment porewater, in Geochemical Transformotions of Sedimentary Sulfur, ACS Symposium Series, vol. 612, edited by A. Vairavamurthy and M. Schoonen, pp. 243-261, ACS, Washington, DC, 1995.

Madsen, O., Sediment transport and coastal processes, Massachusetts Institute of Technology 1.67 Lecture Notes, 1975.

Martens, C., R. Berner, and J. Rosenfeld, Interstitial water chemistry of anoxic Long Island Sound sediments. 2. nutrient regeneration and phosphate removal, Limnology and Oceanography, 23(4), 605$617,1978$.

Martin, J., and D. Windom (Eds.), Ocean margin processes in global change : report of the Dahlem Workshop on Ocean Margin Processes in Global Change, Beriin, 1990, March 18-23/, Physical, Chemical and Earth Science Research Reports, vol. 9, Wiley, Chichester, New York, 1991.

Martin, J., G. Knauer, and R. Gordon, Silver distributions and fluxes in north-east Pacific waters, Nature, 305, 306-309, 1983.

Martin, W., and F. Sayles, Seasonal cycles of particle and solute transport processes in nearshore sediments: $222 \mathrm{Rn} / 226 \mathrm{Ra}$ and $234 \mathrm{Th} / 238 \mathrm{U}$ disequilibrium at a site in Buzzards Bay, MA., Geochimica et Cosmochimica Acta, 51, 927-943, 1987.

McDuff, R., and R. Ellis, Determining diffusion coefficients in marine sediments: a laboratory study of the validity of resistivity techniques, American Journal of Science, 279, 666-675, 1979.

McKay, J., and T. Pedersen, Geochemical behavior of redox-sensitive trace metals in iron-sulfide layers, EOS, 81, F613, 2000.

McNeil, J., K. Taylor, and W. Lick, Measurements of erosion of undisturbed bottom sediments with depth, Journal of Hydraulic Engineering-ASCE, 6(122), 316-324, 1986.

Millero, F., Thermodynamics of the carbon dioxide system in the oceans, Geochimica et Cosmochimica Acta, 59(4), 661-677, 1995.

Moens, L., F. Vanhaecke, J. Riondato, and R. Dams, Some figures of merit of a new double focusing inductively- coupled plasma-mass spectrometer, Journal of Analytical Atomic Spectrometry, 10(9), 569-574, 1995.

Moffett, J., and L. Brand, Production of strong, extracellular Cu chelators by marine cyanobacteria in response to Cu stress, Limnology and Oceanography, $4 I(3), 388-395,1996$.

Moffett, J., L. Brand, P. Croot, and K. Barbeau, Cu speciation and cyanobacyterial distribution in harbors subject to anthropogenic Cu inputs, Limnology and Oceanography, 42(5), 789-799, 1997.

Mopper, K., and D. Delmas, Trace determination of biological thiols by liquid chromatography and precolumn fluorometric labeling with o-phthalaldehyde, Analytical Chemistry, 56, 2557-2560, 1984.

Morel, F., and J. Hering, Principles and Applications of Aquatic Chemistry, John Wiley and Sons, Inc., New York, 1993.

Morford, J., L. Kalnejais, W. Martin, R. Francois, and I.-M. Karle, Sampling marine porewaters for $\mathrm{Mn}, \mathrm{Fe}, \mathrm{U}, \mathrm{Re}$ and Mo: modifications on diffusional equilibration thin film gel probes, Journal of Experimental Marine Biology and Ecology, 285-286, 85-103, 2003.

Morin, J., and J. Morse, Ammonium release from resuspended sediments in the Laguna Madre Estuary, Marine Chemistry, 65(1-2), 97-110, 1999.

Morse, J., Interaction of trace metals with authigenic sulfide minerals: implications for their bioavailability, Marine Chemistry, 46, 1-6, 1994.

Morse, J., and T. Arakaki, Adsorption and coprecipitation of divalent metals with mackinawite (FeS), Geochimica et Cosmochimica Acta, 57(15), 3635-3640, 1993.

Myers, C., and K. Nealon, Microbial reduction of manganese oxides: Interactions with iron and sulfur, Geochimica et Cosmochimica Acta, 52, 2727-2732, 1988. 
Ndung'u, K., M. Thomas, and A. Flegal, Silver in the western equatorial and south Atlantic Ocean, Deep-Sea Research. Part II: Topical Studies in Oceanography, 48, 2933-2945, 2001.

Nieboer, E., and D. Richardson, The replacement of the nondescript term 'heavy metals' by a biologically and chemically significant classification of metal ions, Environmental Pollution (Series B), 1 , $3-26,1980$.

Niu, H., and R. Houk, Fundamental aspects of ion extraction in inductively coupled plasma mass spectrometry, Spectrochimica Acta Part. B: Atomic Spectroscopy, 51(8), 779-815, 1996.

Ong, C., and J. Leckie, Surface and solution speciation of $\mathrm{Ag}(\mathrm{i})$ in a heterogeneous ferrihydrite solution system with thiosulfate, in Adsorption of Metals by Geomedia, edited by E. Jenne, pp. 317-331, Academic Press, 1998.

Pattrick, R., J. Mosselmans, J. Charnock, K. England, G. Helz, C. Garner, and D: Vaughan, The structure of amorphous copper sulfide precipitates: an x-ray absorption study, Geochimica et Cosmochimica Acta, 61, 2023-2036, 1997.

Pawlowicz, R., B. B. Beardsley, and S. Lentz, Classical tidal harmonic analysis including error estimates in matlab using T_TIDE, Computers and Geosciences, 28, 929-937, 2002.

Petersen, W., E. Willer, and W. C., Remobilization of trace elements from polluted anoxic sediments after resuspension in oxic water, Water, Air and Soil Pollution, 99, 515-522, 1997.

Poitrasson, F, and A. Dundas, Direct isotope ratio measurement of ultra-trace lead in waters by double focusing inductively coupled plasm mass spectormetry with an ultrasonic nebuliser and a desolvation unit, Journal of Analytical Atomic Spectrometry, 14, 1573-1577, 1999.

Ranville, M., and A. Flegal, Silver in the North Pacific Ocean, Geochemistry Geophysics Geosystems, 6(Q03M01), 2005.

Ratte, H., Bioaccumulation and toxicity of silver compounds: A review, Environmental Toxicology and Chemistry, 18(1), 89-108, 1999.

Ravens, T., Sediment resuspension in Boston Harbor, Ph.D. thesis, Massachusetts Institute of Technology, 1997.

Ravens, T., and P. Gschwend, Flume measurements of sediment erodibility in Boston Harbor, Journal of Hydraulic Engineering-Asce, 125(10), 998-1005, 1999.

Ravizza, G., and M. Bothner, Osmium isotopes and silver as tracers of anthropogenic metals in sediments from Massachusetts and Cape Cod Bays, Geochimica et Cosmochimica Acta, 60(15), 27532763, 1996.

Reuer, M., and D. Weiss, Anthropogenic lead dynamics in the terrestrial and marine environment, Philosophical Transactions of the Royal Society of London Series a-Mathematical Physical and Engineering Sciences, 360(1801), 2889-2904, 2002.

Rivera-Duarte, I., and A. Flegal, Benthic lead fluxes in San Francisco Bay, California, USA, Geochimica et Cosmochimica Acta, 58, 3307-3313, 1994.

Rivera-Duarte, I., and A. Flegal, Microtechniques for the determination of nanomolar concentrations of trace elements in less then $10 \mathrm{mls}$ of sediment porewater, Analytica Chimica Acta, 328, 13-17, 1996.

Rivera-Duarte, I., and A. Flegal, Pore-water silver concetration gradients and benthic fluxes from contaminated sediments of San Francisco Bay, California,USA, Marine Chemistry, 56, 15-26, $1997 \mathrm{a}$.

Rivera-Duarte, I., and A. Flegal, Porewater gradients and diffusive benthic fluxes of $\mathrm{Co}, \mathrm{Ni}, \mathrm{Cu}, \mathrm{Zn}_{\mathrm{n}}$ and Cd in San Francisco Bay, Croatica Chimica Acta, 70(1), 389-417, 1997b.

Rodushkin, I., and T. Ruth, Determination of trace metals in estuarine and sea water reference materials using high resolution inductively coupled plasma mass spectrometry, Joumal of Analytical Atomic Spectrometry, 12, 1181-1185, 1997.

Roekens, E., and R. V. Grieken, Kinetics of iron(ii) oxidation in seawater at various pH, Marine Chemistry, 13, 195-202, 1983.

Rosenthal, Y., P. Lam, E. Boyle, and J. Thomson, Authigenic cadmium enrichments in suboxic sediments: Precipitation and postdepositional mobility, Earth and Planetary Science Letters, 192(1-4), 99-111, 1995.

Rosman, K., and P. Taylor, Isotopic compositions of the elements 1997, Pure and Applied Chemistry, 71, 1593-1607, 1999. 
Rozan, T., M. Lassman, D. Ridge, and G. Luther, Evidence for iron, copper and zinc complexation as multinuclear sulphide clusters in oxic rivers, Nature, 406, 879-882, 2000a.

Rozan, T., S. Theberge, and G. Luther, Quantifying elemental sulfur (S-0), bisulfide (HS ${ }^{-}$) and polysulfides $\left(\mathrm{S}_{x}^{2-}\right)$ using a voltammetric method, Analytica Chimica Acta, 415(1-2), 175-184, $2000 \mathrm{~b}$.

Rozan, T., G. Luther, D. Ridge, and S. Robinson, Determination of $\mathrm{Pb}$ complexation in oxic and sulfidic water using pseudovoltammetry, Environmental Science and Technology, 37, 3845-3852, 2003.

Rue, E., and K. Bruland, The role of organic complexation on ambient iron chemistry in the equatorial Pacific Ocean and the response of a mesoscale iron addition experiment, Limnology and Oceanography, 42(5), 901-910, 1997.

Saito, M., D. Sigman, and F. Morel, The bioinorganic chemistry of the ancient ocean: the co-evolution of cyanobacterial metal requirements and biogeochemical cycles at the archean-proterozoic boundary?, Inorganica Chimica Acta, 356, 308-318, 2003.

Sanford, L., W. Panageotou, and J. Halka, Tidal resuspension of sediments in northern Chesapeake Bay, Marine Geology, 97, 87-103, 1991.

Santschi, P., P. Hohener, G. Benoit, and M. Brink, Chemical processes at the sediment-water interface, Marine Chemistry, 30, 269-315, 1990.

SanudoWilhelmy, S., I. RiveraDuarte, and A. Flegal, Distribution of colloidal trace metals in the San Francisco Bay estuary, Geochimica et Cosmochimica Acta, 60(24), 4933-4944, 1996.

Saulnier, I., and A. Mucci, Trace metal remobilization following the resuspension of estuarine sediments; Saguenay Fjord, Canada, A pplied Geochemistry, 15(2), 191-210, 2000.

Sawlan, J., and J. Murray, Trace metal remobilization in the interstitial waters of red clay and hemipelagic marine sediments, Earth and Planetary Science Letters, 64(2), 213-230, 1983.

Schwarzenbach, R., P. Gschwend, and D. Imboden, Environmental Organic Chemistry, 2nd edition ed., Wiley-Interscience, New York, NY, 2003.

Shafer, M., S. Hoffmann, J. Overdier, and D. Armstrong, Physical and kinetic speciation of copper and zinc in three geochemcally contrasting marine estuaries, Environmental Science and Technology, 38 (14), 3810-3819, 2004.

Shank, G., S. Skrabal, R. Whitehead, and R. Kieber, Fluxes of strong Cu-complexing ligands from sediments of an organic-rich estuary, Estuarine Coastal and Shelf Science, 60(2), 349-358, 2004.

Shaw, $\mathrm{T}$., J. Gieskes, and R. Jahnke, Early diagenesis in differing depositional-environments - the response of transition-metals in pore water, Geochimica et Cosmochimica Acta, 54(5), 1233-1246, 1990.

Shea, D., and G. Helz, The solubility of copper in sulfidic waters: sulfide and polysulfide complexes in equilibrium with covellite, Geochimica et Cosmochimica Acta, 52, 1815-1825, 1988.

Shea, D., and W. Maccrehan, Role of biogenic thiols in the solubility of sulfide minerals, Science of the Total Environment, 73(1-2), 135-141, 1988.

Shen, G., and E. Boyle, Lead in corals: reconstruction of historical industrial fluxes to the surface ocean., Earth and Planetary Science Letters, 82, 289-304, 1987.

Shimeta, J., and J. Sisson, Taxon-specific tidal resuspension of protists into the subtidal benthic boundary layer of a coastal embayment, Marine Ecology-Progress Series, 177, 51-62, 1999.

Shimeta, J., C. Amos, S. Beaulieu, and O. Ashiru, Sequential resuspension of protists by accelerating tidal flow: Implications for community structure in the benthic boundary layer, Limnology and Oceanography, 47(4), 1152-1164, 2002.

Signell, R., and B. Butman, Modeling tidal exchange and dispersion in Boston Harbor, Journal of Geophysical Research-Oceans, 97(C10), 15,591-15,606, 1992.

Signell, R., and J. List, Effect of wave-enhanced bottom friction on storm driven circulation in Massachusetts Bay, Journal of Waterway, Port, Coastal and ocean Engineering, September/October, 233-239, 1997.

Signell, R., H. Jenter, and A. Blumberg, Predicting the physical effects of relocating Boston's sewage outfall, Estuarine Coastal and Shelf Science, 50(1), 59-71, 2000.

Simpson, S., S. Apte, and G. Batley, Effect of short-term resuspension events on trace metal speciation in polluted anoxic sediments, Environmental Science and Technology, 32(5), 620-625, 1998. 
Skrabal, S., J. Donat, and D. Burdige, Fluxes of copper-complexing ligands from estuarine sediments, Limnology and Oceanography, 42(5), 992-996, 1997.

Skrabal, S., J. Donat, and D. Burdige, Pore water distributions of dissolved copper and coppercomplexing ligands in estuarine and coastal marine sediments, Geochimica et Cosmochimica Acta, 64(11), 1843-1857, 2000.

Smith, D., R. Bell, and J. Kramer, Metal speciation in natural waters with emphasis on reduced sulfur groups as strong metal binding sites, Comparative Biochemistry and Physiology C-Toxicology and Pharmacology, 133(1-2), 65-74, 2002.

Sorensen, J., and B. Jorgensen, Early diagenesis in sediments from Danish coastal waters: Microbial activity and Mn-Fe-S geochemistry, Geochimica et Cosmochimica Acta, 51, 1583-1590, 1987.

Squire, S., G. Scelfo, J. Revenaugh, and A. Flegal, Decadal trends of silver and lead contamination in San Francisco Bay surface waters, Environmental Science and Technology, 36(11), 2379-2386, 2002.

Stumm, W., and J. Morgan, Aquatic Chemistry, 3rd edition ed., Wiley-Interscience, New York, 1981.

Sukola, K., F. Wang, and A. Tessier, Metal-sulfide species in oxic waters, Analytica Chimica Acta, 528(2), 183-195, 2005.

Sunda, W., Trace metal-phytoplankton interactions in the sea, in Chemistry of Aquatic Systems: Local and Global Perspectives, edited by G. Bidoglio and W. Stumm, pp. 213-247, Springer, 1994.

Sunda, W., and S. Huntsman, Microbial oxidation of manganese in a North Carolina estuary, Limnology and Oceanography, 32(3), 552-564, 1987.

Sundby, B., L. Anderson, P. Hall, A. Iverfeldt, M. Vanderloeff, and S. Westerlund, The effect of oxygen on release and uptake of cobalt, manganese, iron and phosphate at the sediment-water interface, Geachimica et Cosmochimica Acta, 50(6), 1281-1288, 1986.

Taillefert, M., C.-P. Lienemann, J.-F. Gaillard, and D. Perret, Speciation, reactivity, and cycling of $\mathrm{Fe}$ and $\mathrm{Pb}$ in a meromictic lake, Geochimica et Cosmochimica Acta, 64(2), 169-183, 2000.

Tang, D., C. Hung, K. Warnken, and P. Santschi, The distribution of biogenic thiols in surface waters of Galveston Bay, Limnology and Oceanography, 45(6), 1289-1297, 2000a.

Tang, D., L. Wen, and P. Santschi, Analysis of biogenic thiols in natural water samples by high-performance liquid chromatographic separation and fluorescence detection with ammonium 7-fluorobenzo2-oxa-1,3-diazole-4- sulfonate (SBD-F), Analytica Chimica Acta, 408(1-2), 299-307, 2000b.

Tang, D., M. Shafer, K. Vang, D. Kamer, and D. Amstrong, Determination of dissolved thiols using solid-phase extraction and liquid chromatographic determination of fluorescently derivatized thiolic compounds, Journal of Chromatography A, 998(1-2), 31-40, 2003.

Tengberg, A., E. Almroth, and P. Hall, Resuspension and its effects on organic carbon recycling and nutrient exchange in coastal sediments: in situ measurements using new experimental technology, Journal of Experimental Marine Biology and Ecology, 285, 119-142, 2003.

Tessier, A., D. Fortin, N. B. R. Devitre, and G. Leppard, Metal sorption to diagenetic iron and manganese oxyhydroxides and associated organic matter: Narrowing the gap between field and laboratory measurements, Geochimica et Cosmochimica Acta, 60(3), 387-404, 1996.

Thamdrup, B., Bacterial manganese and iron reduction in aquatic sediments, Advances in Microbial Ecology, 16, 4l-84, 2000.

Thomsen, L., and G. Gust, Sediment erosion thresholds and characteristics of resuspended aggregates on the western European continental margin, Deep-Sea Research Part I-Oceanographic Research Papers, 47(10), 1881-1897, 2000.

Thomson, J., N. Higgs, I. Croudace, S. Colley, and D. Hydes, Redox zonation at an oxic/post-oxic boundary in deep sea sediments, Geochimica et Cosmochimica Acta, 57, 579-595, 1993.

Tolhurst, T., R. Riethmuller, and D. Paterson, In situ versus laboratory analysis of sediment stability from intertidal mudflats, Continental Shelf Research, 20(10-11), 1317-1334, 2000.

Tsai, C.-H., and W. Lick, A portable device for measuring sediment resuspension, Journal of Great Lakes Research, 12, 314-321, 1986.

Vairavamurthy, A., and K. Mopper, Field method for determination of traces of thiols In naturalwaters, Analytica Chimica Acta, 236(2), 363-370, 1990. 
Vairavamurthy, M., and K. Mopper, Geochemical formation of organosulfur compounds (thiols) by addition of $\mathrm{H}_{2} \mathrm{~S}$ to sedimentary organic matter, Nature, 329, 623-625, 1987.

Vanhaecke, F., L. Moens, R. Dams, I. Papadakis, and P. Taylor, Applicability of high-resolution ICP mass spectrometry for isotope ratio measurements, Analytical Chemistry, 69(2), 268-273, 1997.

Vetter, R., P. Matrai, B. Javor, and J. O'Brien, Reduced sufur compounds in the marine environment: Analysis by HPLC, in Biogenic Sulfur in the Environment, ACS Symposium Series, vol. 393, edited by E. Saltzman and W. Cooper, pp. 243-261, ACS, Washington, DC, 1987.

Vojak, P., C. Edwards, and M. Jones, Evidence for microbiological manganese oxidation in the River Tamar Estuary, South West England, Estuarine and Coastal and Shelf Science, 20, 661-671, 1985.

Ward, N., Trace elements, in Environmental Analytical Chemistry, edited by F. Fifield and P. Haines, pp. 320-350, Chapman and Hall, 1995.

Warnken, K., G. Gill, L. Griffin, and P. Santschi, Sediment-water exchange of $\mathrm{Mn}, \mathrm{Fe}$, Ni and $\mathrm{Zn}$ in Galveston Bay, Texas, Marine Chemistry, 73(3-4), 215-231, 2001.

Wen, L., P. Santschi, G. Gill, C. Paternostro, and R. Lehman, Colloidal and particulate silver in river and estuarine waters of Texas, Envronmental Science and Technology, 31(3), 723-731, 1997 :

Westerlund, S., L. Anderson, P. Hall, A. Iverfeldt, M. Vanderloeff, and B. Sundby, Benthic fluxes of cadmium, copper, nickel, zinc and lead in the coastal environment, Geochimica et Cosmochimica Acta, $50(6), 1280-1296,1986$.

Wheatcroft, R. A., I. Olmez, and F. X. Pink, Particle bioturbation in Massachusetts Bay: Preliminary results using a new deliberate tracer technique, Joumal of Marine Research, 52(6), 1129-1150, 1994.

Williams, M., G. Millward, M. Nimmo, and G. Fones, Fluxes of $\mathrm{Cu}, \mathrm{Pb}$ and $\mathrm{Mn}$ to the north-eastern Irish Sea: The importance of sedimental and atmospheric inputs, Marine Pollution Bulletin, $36(5)$, $366-375,1998$.

Wu, J., and E. Boyle, Lead in the Western North Atlantic: Completed response due to gaseoline phaseout, Geochimica et Cosmochimica Acta, 61(15), 3279-3283, 1997.

$\mathrm{Xu}, \mathrm{Y}_{\text {., }}$ and W. Wang, Silver uptake by a marine diatom and its transfer to the coastal copepod acartia spinicauda, Environmental Toxicology and Chemistry, 29(3), 682-690, 2004.

Yoo, H., J.-S. Lee, B.-G. Lee, I. Lee, C. Schlekat, C.-H. Koh, and S. Luoma, Uptake pathway for Ag bioaccumulation in three benthic invertebrates exposed to contaminated sediments, Marine EcologyProgress Series, 270, 141-152, 2004.

Young, R., Flow and sediment properties influencing erosion of fine-grained marine sediments : sea floor and laboratory experiments., Ph.D. thesis, MIT-WHOI, 1975.

Zhang, H., W. Davison, R. Mortimer, M. Krom, P. Hayes, and I. Davies, Localised remobilization of metals in a marine sediment, Science of the Total Environment, 296(1-3), 175-187, 2002.

Zhang, Y., H. Amakawa, and Y. Nozaki, Oceanic profiles of dissolved silver: precise measurements in the basins of western North Pacific, Sea of Okhotsk, and the Japan Sea, Marine Chemistry, 75(1-2), 151-163, 2001. 